UNIVERSIDADE DE SÃO PAULO

Instituto de Física

\title{
Estudo Analítico das Probabilidades de Oscilação de Neutrinos na Matéria em Três Gerações
}

\author{
Caio Augusto Pelegrina Del Bianco Licciardi
}

Orientadora: Profa. Dra. Renata Zukanovich Funchal

Dissertação apresentada ao Instituto de Física da Universidade de São Paulo para a obtenção do título de Mestre em Ciências

\section{Comissão examinadora}

Profa. Dra. Renata Zukanovich Funchal (IF-USP)

Prof. Dr. Gustavo Alberto Burdman (IF-USP)

Prof. Dr. Alberto Martin Gago Medina (PUC del Peru)

São Paulo 

Aos meus pais e minha família 



\section{Agradecimentos}

À Renata, minha orientadora, pela dedicação e confiança depositadas em mim durante todo o período do meu projeto de Mestrado, e por sua forma de trabalho que entusiasma a pesquisa em física.

Aos Professores Gustavo Burdman e Oscar Éboli pela cooperação, auxílio e aulas bem lecionadas durante minha formação.

Aos meus pais que sempre me apoiaram nos estudos e incentivaram imensamente a realização de meus objetivos.

Ao meu irmão Paulo e sua esposa Lívia pelas excelentes acomodações fornecidas no período final de trabalho.

Ao meu irmão Paco e aos meus amigos pelo companheirismo e diversão proporcionados neste período. Entre eles se destacam o Rone, Pedro, Silas, Alan, Felipe, Taarik, Eduardo, Marcelos e meu primo Guilherme.

À Ana Carolina, minha querida namorada, pela compreensão, carinho, respeito e amor demonstrados nestes dois anos em que estamos juntos.

Finalmente, gostaria de agradecer à todos que direta ou indiretamente me ajudaram a realizar este trabalho.

À FAPESP, pelo apoio financeiro e respeito que guarda aos seus bolsistas. 


\section{Resumo}

Neste projeto realizamos um estudo sobre as fórmulas de probabilidades de oscilação de neutrinos na matéria em duas e três gerações. Estudamos extensivamente a fenomenologia e os experimentos de oscilação de neutrinos. Além de rever todas as expressões analíticas para as soluções exatas e aproximadas conhecidas na literatura para as probabilidades de conversão, também desenvolvemos soluções novas. Mostramos que os mesmos potenciais que possuem solução exata em duas gerações, também possuem em três. Com o formalismo proposto nesta dissertação para resolução da equação de evolução dos neutrinos na matéria, fica descartada a existência de outros potenciais, que não os aqui discutidos, com solução analítica em termos de funções que são obtidas como casos especiais ou limites da função hipergeométrica generalizada. 


\section{Abstract}

In this project we realized a study about the formulas of neutrino oscillation probabilities in matter in two and three generations. We studied the neutrino oscillation phenomenology and its experiments. We reviewed all the analytic expressions to the exact and aproximate solutions known at the literature to the conversion probabilities, as well as we have developed new ones. We showed that the same potentials which have exact solutions in two generations, also have exact solutions in three generations. By using the proposed formalism in this dissertation to the resolution of the evolution equation of neutrinos in matter, we ruled out the existence of other potentials not discussed here that have exact solution in terms of the special functions that are special cases or limits of the generalized hypergeometric function. 


\section{Sumário}

Introdução

1 História dos neutrinos e o Modelo Padrão 5

1.1 Problema do decaimento $\beta \ldots \ldots \ldots 5$

1.1.1 O átomo no começo do século XX . . . . . . . . . . 6

1.1.2 Soluções desesperadas . . . . . . . . . . . . . . 7

1.1.3 Observação experimental . . . . . . . . . . . . 7

1.2 Da teoria de Fermi à teoria Eletrofraca . . . . . . . . . . . . . . 8

1.2.1 Teoria de Fermi . . . . . . . . . . . . . . . 8

1.2.2 Violação da paridade e helicidade do neutrino . . . . . . . 9

1.2.3 Teoria Eletrofraca . . . . . . . . . . . . . . . . . 11

1.3 Neutrinos no Modelo Padrão . . . . . . . . . . . . . . . . . . . 12

1.3.1 Interações no Modelo Padrão . . . . . . . . . . . . . . . 14

1.3.2 Número de famílias . . . . . . . . . . . . . . . . . . . . . . 14

1.3.3 Massas e testes cinemáticos . . . . . . . . . . . 15

1.3.4 Duplo decaimento $\beta$. . . . . . . . . . . . . . . 16

1.4 Neutrinos além do Modelo Padrão . . . . . . . . . . . . . . . . 16

1.4.1 Problema do neutrino solar . . . . . . . . . . . . . . . 17

1.4.2 Oscilação de neutrinos . . . . . . . . . . . . . . . 17

1.4.3 Neutrinos massivos . . . . . . . . . . . . . . 17

1.4.4 Duplo decaimento $\beta$ sem neutrinos . . . . . . . . . . 18

1.5 Cronograma histórico . . . . . . . . . . . . . . . . . . . . . . . 18

2 Fontes de neutrinos e métodos de observação 21

2.1 Fontes naturais . . . . . . . . . . . . . . . . . 21

2.1.1 Neutrinos solares . . . . . . . . . . . . . . . . 22

2.1.2 Neutrinos atmosféricos . . . . . . . . . . . . . . 24

2.1.3 Neutrinos de supernovas . . . . . . . . . . . . . 26

2.1.4 Geoneutrinos . . . . . . . . . . . . . . . 27

2.1.5 Neutrinos cosmológicos . . . . . . . . . . . . . . . . . . 28

2.2 Fontes humanas . . . . . . . . . . . . . . . . . . . . . . . . . 28 
2.2.1 Neutrinos de aceleradores . . . . . . . . . . . . . . 29

2.2.2 Neutrinos de reatores . . . . . . . . . . . . . . . . . 31

2.3 Métodos de observação . . . . . . . . . . . . . . . . . 33

2.3.1 Detecção radioquímica . . . . . . . . . . . . . . . . . 33

2.3.2 Radiação Cherenkov . . . . . . . . . . . . . . 34

2.3.3 Contagem em líquido cintilante . . . . . . . . . . . 35

2.3.4 Calorímetros . . . . . . . . . . . . . . . 37

2.4 Resumo . . . . . . . . . . . . . . . . . . 38

2.4.1 Fontes de neutrinos . . . . . . . . . . . . . . . . . 38

2.4.2 Métodos de deteç̧ão . . . . . . . . . . . . . . . . . . 38

3 Experimentos de neutrinos $\quad 39$

3.1 Experimentos de neutrinos solares . . . . . . . . . . . . . . . . 39

3.1.1 Homestake: experimentos com cloro . . . . . . . . . . . . . 40

3.1.2 SAGE e GALLEX/GNO: experimentos com gálio . . . . . 41

3.1.3 Kamiokande/SK: experimentos com água . . . . . . . . . . 41

3.1.4 SNO: experimentos com água pesada . . . . . . . . . . . 43

3.1.5 Futuros experimentos: baixas energias . . . . . . . . . 43

3.1.6 Resumo dos resultados . . . . . . . . . . . . . . . . . . . 44

3.2 Experimentos de neutrinos em reatores . . . . . . . . . . . 45

3.2.1 ILL, Bugey, Gosgen, Krasnoyarsk, Rovno, Savannah River: detectores de SBL . . . . . . . . . . . . . . . . 46

3.2.2 CHOOZ, KamLAND, Palo Verde: detectores de LBL . . . 46

3.2.3 Futuros experimentos: detectores distantes . . . . . . . . . 47

3.2.4 Resumo dos resultados . . . . . . . . . . . . . . . . . . 48

3.3 Experimentos de neutrinos atmosféricos . . . . . . . . . . . . . . . 49

3.3.1 Experimentos com calorímetros: KGF, Fréjus, Baksan, Soudan 2, NUSEX, MACRO e MINOS . . . . . . . . . . 52

3.3.2 Experimentos com água: Kamiokande/SK e IMB . . . . . 53

3.3.3 Futuros experimentos: grandes calorímetros e detectores Cherenkov de megatoneladas . . . . . . . . . . . . 55

3.3.4 Resumo dos resultados . . . . . . . . . . . . . . . 55

3.4 Experimentos de neutrinos em aceleradores . . . . . . . . . . . 56

3.4.1 Experimentos de SBL . . . . . . . . . . . 57

3.4.2 Experimentos de LBL: K2K, MINOS e OPERA . . . . . . 59

3.4.3 Futuros experimentos: superfeixes de neutrinos . . . . . . 60

3.4.4 Resumo dos resultados . . . . . . . . . . . . . . . 60 
4 Análise dos parâmetros de oscilação $\quad 61$

4.1 Oscilação de neutrinos . . . . . . . . . . . . . . . . . 61

4.1.1 Abordagem convencional . . . . . . . . . . . . . . . 61

4.1.2 Oscilação de neutrinos monoenergéticos . . . . . . . . . . . 62

4.1.3 Fórmulas em duas gerações . . . . . . . . . . . . . . . 63

4.1.4 Efeitos com distribuição energética . . . . . . . . . . . . 64

4.1.5 Efeitos de matéria . . . . . . . . . . . . . . 66

4.2 Determinação dos parâmetros de oscilação . . . . . . . . . . . . . 68

4.2.1 Análises gerais . . . . . . . . . . . . . . . . . 69

4.2 .2 Análises precisas . . . . . . . . . . . . . . . . 70

4.3 Resumo da análise dos resultados . . . . . . . . . . . . . . . 75

$\begin{array}{lll}5 & \text { Mecanismos de geração de massa } & 77\end{array}$

5.1 O método dos diagramas de sabor . . . . . . . . . . . . . . 77

5.2 O Modelo Padrão implica massa nula para neutrinos . . . . . . . 79

5.3 Expansão no setor fermiônico . . . . . . . . . . . . . . 80

5.3.1 Termo de massa de Dirac . . . . . . . . . . . . . 80

5.3.2 Termos de massa de Majorana . . . . . . . . . . . . . . . 81

5.3.3 Termos de massa Dirac-Majorana e mecanismo de gangorra 81

5.4 Expansão no setor escalar . . . . . . . . . . . . . . . . . . . 82

5.4.1 Modelo com um tripleto . . . . . . . . . . . . 82

5.4 .2 Modelo de Zee . . . . . . . . . . . . . . . . . 85

5.4 .3 Modelo de Zee-Babu . . . . . . . . . . . . . . . . 87

5.5 Expansão nos dois setores . . . . . . . . . . . . . . . . . . . 88

5.6 Massas e oscilação . . . . . . . . . . . . . . . . . . . . 88

6 Equação de evolução na matéria $\quad 91$

6.1 Efeito de matéria . . . . . . . . . . . . . . . . . . . . . . . . 91

6.1.1 Interações de correntes carregadas . . . . . . . . . . . . . . 92

6.1.2 Interações de correntes neutras . . . . . . . . . . . . . . . 93

6.2 Equação efetiva de Schrödinger . . . . . . . . . . . . . . . . 93

6.3 Parametrização da matriz de mistura . . . . . . . . . . . . . . 95

6.3.1 Número de graus de liberdade de $U(n)$ e $S U(n) \ldots \ldots$. . . 96

6.3 .2 Matrizes de $S U(2)$ e $S U(3) \quad \ldots \ldots$. . . . . . . . . . . . 96

6.3.3 Fases de Dirac e Majorana em $U(n)$ e $S U(n)$. . . . . . . 97

6.3.4 Redefinição de fases em $S U(2)$ e $S U(3)$. . . . . . . . . . 98

6.3.5 Parametrização de $U$ em duas gerações . . . . . . . . . . . 99

6.3.6 Parametrização de $U$ em três gerações . . . . . . . . . . . 99

6.4 Propriedades das soluções . . . . . . . . . . . . . . . . . . 100 
6.4.1 Operador de evolução . . . . . . . . . . . . . . 100

6.4.2 Probabilidades independentes . . . . . . . . . . . . 101

6.4.3 Hierarquia de massas . . . . . . . . . . . . . . . . . . . . . 102

7 Soluções exatas da equação de evolução 105

7.1 Método de resolução . . . . . . . . . . . . . . . . 106

7.1.1 Equação de evolução . . . . . . . . . . . . . . . . . . . . 106

7.1.2 Formalismo . . . . . . . . . . . . . . . . 107

7.2 Resultados exatos em duas gerações . . . . . . . . . . . . . . 109

7.2.1 Caso constante . . . . . . . . . . . . . . . . 110

7.2.2 Caso exponencial generalizado . . . . . . . . . . . . . . 112

7.2.3 Caso linear generalizado . . . . . . . . . . . . . . . . . 117

7.3 Resultados exatos em três gerações . . . . . . . . . . . . . . . . 120

7.3.1 Caso constante . . . . . . . . . . . . . . . . 121

7.3.2 Caso exponencial generalizado . . . . . . . . . . . . . 122

7.3.3 Caso linear generalizado . . . . . . . . . . . . . . . . . 123

7.4 Outros métodos - potencial constante . . . . . . . . . . . . . . 123

7.4.1 Autovalores efetivos na matéria . . . . . . . . . . . . . . 124

7.4.2 Teorema de Cayley-Hamilton . . . . . . . . . . . . . . 125

7.4.3 Método de Kimura-Takamura-Yokomakura . . . . . . . . . 127

8 Soluções aproximadas da equação de evolução 129

8.1 Motivações . . . . . . . . . . . . . . . . . . . . . . . 129

8.1.1 Complexidade das fórmulas exatas . . . . . . . . . . 130

8.1.2 Redução de 3 gerações para 2 gerações . . . . . . . . . . . 130

8.2 Métodos de aproximação . . . . . . . . . . . . . . . . . . . 132

8.2.1 Expansão perturbativa do operador de evolução . . . . . . 132

8.2 .2 Aproximação adiabática . . . . . . . . . . . . . . 133

8.2.3 Outros métodos com expansão em série . . . . . . . . . . . 134

8.3 Aproximação adiabática . . . . . . . . . . . . . . . . . . 135

8.3.1 Solução adiabática . . . . . . . . . . . . . . . 135

8.3.2 Feixe de neutrinos com distribuição energética . . . . . . . 136

8.3.3 Correções à aproximação adiabática . . . . . . . . . . . . . 137

8.3.4 Ansatz de Kuo e Pantaleone . . . . . . . . . . . . . . . . . 140

8.3.5 Aproximação adiabática e o efeito MSW . . . . . . . . . . 140

8.3.6 Aplicação em três gerações . . . . . . . . . . . . . . . . . . 141

8.4 Aproximação fora da zona de ressonância . . . . . . . . . . . . . . 142

8.5 Aproximações em três gerações . . . . . . . . . . . . . . . . . 143

$\begin{array}{lr}\text { Conclusões e perspectivas } & 145\end{array}$ 
$\begin{array}{ll}\text { A Sobre a helicidade e a quiralidade de partículas } & 149\end{array}$

B Neutrinos de Majorana $r 151$

B.1 Definição do campo de Majorana . . . . . . . . . . . . . . 151

B.2 Propriedades . . . . . . . . . . . . . . . 152

B.3 Propagador de Majorana . . . . . . . . . . . . . . . 152

B.4 Termos de massa para neutrinos . . . . . . . . . . . . . 153

$\begin{array}{ll}\text { C Glossário } & 155\end{array}$

$\begin{array}{ll}\text { Referências Bibliográficas } & 157\end{array}$ 


\section{Introdução}

A física de neutrinos se apresenta hoje como uma das janelas mais promissoras para física além do Modelo Padrão. O principal motivo disto é que o setor de neutrinos oferece condições excepcionais para o estudo de questões fundamentais em física de partículas pois, ao contrário dos quarks, neutrinos não participam das interações fortes. Além disso, a descoberta das oscilações de neutrinos nos últimos anos confirmada por experimentos com neutrinos solares, atmosféricos, de aceleradores e de reatores tornou a física de neutrinos um dos campos mais excitantes e ativos da física de partículas.

Até o momento uma grande quantidade de informação já foi obtida sobre as propriedades dos neutrinos. Estas informações fornecem evidências sobre a existência de massa para neutrinos, que até pouco tempo eram consideradas nulas pelo Modelo Padrão. Isto implica em mudanças na física de partículas, com consequências importantes em outras áreas. O estudo aprofundado de neutrinos pode nos mostrar caminhos para melhor entendimento da nucleosíntese primordial que depende sensivelmente das interações desta partícula. Neutrinos com massa $m_{\nu} \sim 1 \mathrm{eV}$ têm um importante papel na formação de galáxias.

Tudo isto nos dá apenas uma breve motivação para começar um estudo em neutrinos. Particularmente, o fenômeno de oscilação tem sido uma importante ferramenta na última década para o estudo das propriedades dos neutrinos. Apesar do progresso realizado, ainda permanecem muitas questões em aberto, sobretudo porque alguns parâmetros de oscilação ainda não são bem estabelecidos, como a fase de violação da simetria CP ou mesmo a hierarquia de massa (não sabemos se a terceiro autovalor de massa está acima ou abaixo do primeiro). Ainda não sabemos também se os neutrinos são suas próprias antipartículas. Muitas dessas questões poderão ser respondidas, em princípio, pela observação do fenômeno de oscilação. Para tanto é necessário compreender a conversão de sabor como um fenômeno de três gerações genuínas.

As fórmulas de probabilidade de transição dependem de um conjunto de parâmetros a serem medidos. O conjunto total destes parâmetros que descrevem a evolução dos autoestados de sabor em um cenário com três famílias de neutrinos

é: duas diferenças de massas quadradas $\left(\Delta m_{21}^{2}\right.$ e $\left.\Delta m_{31}^{2}\right)$, três ângulos de mistura 
$\left(\theta_{12}, \theta_{13}\right.$ e $\left.\theta_{23}\right)$ e uma fase de violação $\mathrm{CP}(\delta)$. Recentemente os valores de tais parâmetros, obtidos experimentalmente, tem adquirido uma precisão crescente com incerteza inferior à 20\%. Entretanto, uma próxima geração de experimentos calcula atingir precisão perto de $1 \%$. Isto nos impõe uma necessidade cada vez maior de fórmulas mais precisas.

A presente dissertação é o resultado de um projeto de Mestrado realizado pelo estudante Caio Licciardi no Instituto de Física da Universidade de São Paulo, orientado pela Professora Dra. Renata Zukanovich Funchal.

O objetivo do projeto foi estudar as fórmulas de probabilidade de oscilação de neutrinos no vácuo e na matéria existentes em duas e três gerações. Para alcançar tal objetivo, inicialmente realizamos um estudo detalhado cobrindo uma grande área fenomenológica de neutrinos, incluindo os mecanismos de geração de massa. Além disso, todos os experimentos que de alguma forma evidenciaram o fenômeno de oscilação no setor foram estudados. A partir de então estudamos uma coleção de fórmulas existentes na literatura que descrevem a oscilação de neutrinos. Em apenas alguns casos fórmulas exatas podem ser obtidas quando a trajetória do neutrino passa dentro de um meio material com densidade não nula. Em geral estas fórmulas são relativamente complicadas e difíceis de se tratar. Por isso, a maneira mais fácil de contornar este problema é procurar soluções aproximadas.

Como a procura por fórmulas exatas e/ou compactas é recente, data de duas décadas, acreditamos que esta dissertação é uma das únicas que apresenta uma coleção de tais expressões. Além disso, durante o estudo de soluções exatas, encontramos um formalismo capaz de fornecer todas as soluções conhecidas, e mais algumas, de maneira sistemática tanto em duas como em três gerações. Esta dissertação provê para qualquer aluno com conhecimentos de Mecânica Quântica uma boa introdução ao estudo de oscilações de neutrinos. Evidentemente, algumas passagens requerem conhecimentos em Teoria Quântica de Campos, além é claro, de bases em funções especiais.

A dissertação está organizada como segue. Na primeira parte, que se inicia do Capítulo 1 e vai até o Capítulo 4, fazemos uma introdução detalhada ao estudo de neutrinos. Mais precisamente, no Capítulo 1 apresentamos, ainda que de forma resumida, toda a história da descoberta dos neutrinos e sua relação com a criação do Modelo Padrão.

No Capítulo 2, descrevemos quais as fontes de neutrinos existentes na natureza e quais são aquelas produzidas pelo homem. Discutimos também os métodos de deteç̧ão empregados em experimentos que procuram por neutrinos.

No Capítulo 3, apresentamos todos os experimentos realizados que evidenciaram a oscilação de neutrinos, incluindo experimentos de neutrinos solares, atmosféricos, de reatores e de aceleradores. O objetivo da exaustiva apresen- 
tação é dar um panorâma geral do que já foi feito e em que posição se encontram os experimentos atuais de neutrinos.

No Capítulo 4, discutimos a abordagem de oscilação para a compreensão dos dados experimentais. O foco do capítulo é mostrar onde e como são utilizadas as fórmulas que vamos estudar, e conduzir aos valores experimentais dos parâmetros de oscilação. No final de cada capítulo desta primeira parte produzimos um resumo com as principais informações do capítulo no intuito de enfatizar o conteúdo do mesmo.

Na segunda parte a dissertação toma um rumo mais teórico. No Capítulo 5 apresentamos os principais modelos que se propõem a explicar a geração de massa para os neutrinos. Além de apresentar os dois tipos de termos de massa, Dirac e Majorana, incluímos também exemplos de mecanismos de geração de massa que aparecem em modelos que vão além do Modelo Padrão e que fazem inclusive previsões para os parâmetros de oscilação. No final do capítulo discutimos a influência dos tipos de termos de massa e mecanismos de geração de massa no estudo de oscilações de neutrinos.

No Capítulo 6, demonstramos a equação diferencial que rege a evolução dos neutrinos em condições especiais que abrangem boa parte das oscilações. Uma discussão detalhada da parametrização da matriz de mistura é desenvolvida com o propósito de esclarecer todos os detalhes da equação de evolução e parâmetros da oscilação.

No Capítulo 7, apresentamos as soluções exatas para a equação de evolução através do nosso formalismo, e as comparamos com as existentes na literatura. Apresentamos também métodos que encontram de maneira simplificada as probabilidades de oscilação na matéria com densidade constante em três gerações.

No Capítulo 8, discutimos os métodos de aproximação de soluções existentes e descrevemos quais são os mais utilizadas nas análises experimentais. Ressaltamos, em particular, a aproximação adiabática por ser a fórmula mais utilizada nas análises de neutrinos solares e sendo assim, demonstramos suas peculiaridades.

Nas conclusões discutimos o significado dos resultados obtidos e o que se pôde extrair do projeto. Mostramos também as perspectivas de trabalhos futuros.

No Apêndice A distiguimos os projetores de helicidade e quiralidade para uma partícula massiva, e demonstramos sua equivalência e conservação no caso de partículas sem massa.

No Apêndice B apresentamos o que são neutrinos de Majorana e suas propriedades mais importantes.

A notação seguida na dissertação tentou ser única, mas houve algumas variações entre os capítulos. Contudo, ela não se torna ambígua em nenhum dos 
casos e tais variações só visam simplificar a leitura e compreensão. Todos cálculos foram realizados nas unidades naturais $\hbar=c=1$. 


\section{Capítulo 1}

\section{História dos neutrinos e o Modelo Padrão}

O século XX foi sem dúvida consagrador para a física de partículas. Através de vários problemas e descobertas, o atual Modelo Padrão (MP) foi sendo construído e conseguiu unificar duas forças fundamentais: o eletromagnetismo, que explicava a natureza da luz; e a força fraca, que explicava certo tipo de decaimentos. Entretanto, não existe outra partícula tão importante no desenvolvimento da teoria eletrofraca quanto o neutrino.

A descoberta do neutrino e suas propriedades está intimamente ligada com a necessidade de desenvolver uma teoria nova para explicar os resultados observados nos decaimentos. Neste capítulo apresentamos uma breve introdução histórica à descoberta do neutrino relacionando-o ao desenvolvimento da teoria eletrofraca. Uma descrição histórica bem detalhada pode ser encontrada na Ref. [1], cuja excelente abordagem inspirou a criação deste capítulo.

Na primeira parte é discutido o problema do decaimento $\beta$ e a introdução do neutrino como solução. O modelo de Fermi proposto como uma primeira teoria preditiva é apresentado na sequência. O MP é então introduzido para solucionar os problemas desta teoria. Os neutrinos como são entendidos pelo MP e suas propriedades estão na seção seguinte. A última parte é reservada às motivações para física além do MP através do setor de neutrinos.

\subsection{Problema do decaimento $\beta$}

Interrompida pela Primeira Guerra Mundial, a saga do espectro de raios $\beta$ começou no final do século XIX com os experimentos de Henri Becquerel [2] sobre a radioatividade. Entretanto a primeira observação de que o espectro da radiação $\beta$ era contínuo, e não discreto como se esperava, veio somente em 1914 com um 
experimento realizado por James Chadwick [3]. A Figura 1.1(a) esquematiza seu experimento. Em 1927, Ellis e Wooster confirmaram a continuidade do espectro de $\beta$ [4]. A Figura 1.1(b) apresenta os resultados.

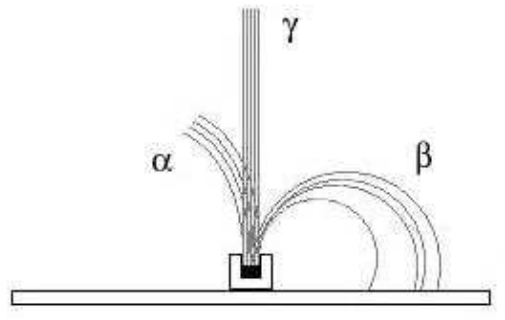

(a)

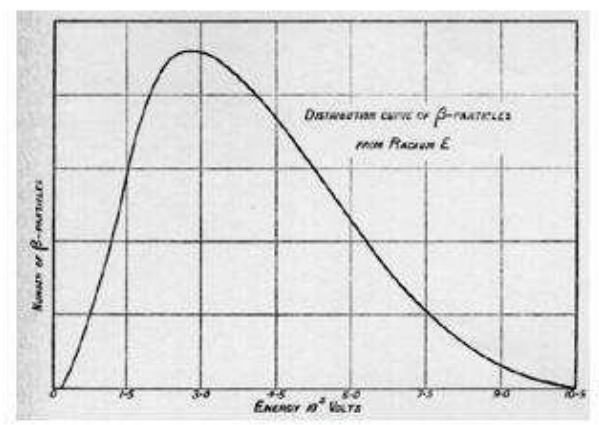

(b)

Figura 1.1: (a) Um simples espectrômetro, onde um campo magnético encurva as trajetórias dos elétrons de uma fonte radioativa. Os diferentes raios de curvatura dependem da energia, assim elétrons com energias diferentes chegam em pontos diferentes do detector, que na época era uma chapa fotográfica. (b) Gráfico obtido por Ellis e Wooster confirmando a continuidade do espectro de energia dos elétrons emitidos [4].

\subsubsection{O átomo no começo do século XX}

O estudo da radiação $\beta$ se tornou parte de um novo campo de descobertas: a física nuclear.

Em 1911 Ernest Rutherford estabeleceu a existência de um pequeno porém massivo núcleo no centro do átomo.

Em 1913 Niels Bohr publicou sua primeira teoria quântica do átomo, discretizando os possíveis valores de energia permitidos para os elétrons circulando o núcleo atômico. Desta forma ele logo concluiu que os elétrons da radiação $\beta$ deveriam ser provenientes do núcleo, pois as energias medidas eram muito altas.

Nesta época outro problema acerca da massa do núcleo era discutido. Como um átomo deve ser eletricamente neutro, para contrabalancear os elétrons nos orbitais, o mesmo número de prótons deveria estar situado no núcleo. Entretanto, a massa encontrada nele era o dobro da massa predita teoricamente. O nêutron não havia sido descoberto até então. Com a inclusão de elétrons no núcleo, a mesma quantidade de prótons poderia ser novamente adicionada e assim obter a massa desejada. Foi neste modelo atômico que o problema do espectro $\beta$ voltou à tona.

Somente em 1932 os físicos começaram a rever o modelo do núcleo. Através da introdução da idéia do nêutron - uma partícula de carga nula, com uma massa 
próxima à do próton e de spin $\frac{1}{2}$ - os elétrons foram definitivamente excluídos do núcleo.

\subsubsection{Soluções desesperadas}

Durante toda a década de 1920, impulsionado pelos sucessos da teoria quântica, Bohr acreditava numa possível não conservação da energia à nível atômico para explicar a continuidade do espectro da radiação $\beta$. Achava que a conservação só era assegurada estatisticamente e que poderia estar nesta não conservação local a origem "misteriosa" da fonte de energia das estrelas. Em julho de 1929 escreveu uma carta para seu amigo Wolfgang Pauli descrevendo suas idéias [5]. Não apreciando estas, Pauli aconselhou o amigo à "manter suas notas guardadas por um bom tempo", porém não apresentava nenhuma outra solução.

Em 1930 Pauli escreveu uma das cartas mais conhecidas da física moderna [6] propondo a existência de uma partícula neutra ainda não observada que deveria estar roubando energia dos elétrons da radiação, tornando o espectro contínuo. Denominou esta solução como "desesperada" para explicar o problema do espectro contínuo.

Após algum tempo, em 1932 Fermi denominou a partícula de Pauli de neutrino para diferenciá-la do nêutron encontrado nos núcleos.

\subsubsection{Observação experimental}

$\mathrm{Na}$ linguagem atual, a proposta de Pauli significava que o decaimento $\beta$ se daria pela seguinte reação [7]:

$$
n \rightarrow p^{+} e^{-} \bar{\nu}
$$

Portanto uma idéia de detecção de neutrinos seria utilizar o chamado decaimento $\beta$ inverso: $\bar{\nu} p^{+} \rightarrow e^{+} n$. Em 1934, utilizando o artigo de Fermi [8] que modelava o decaimento $\beta$, dois físicos alemães Hans Bethe e Rudolf Peierls calcularam a probabilidade desta reação ocorrer por unidade de área [9]. O resultado obtido foi que em um centímetro cúbico de água esta probabilidade é de $7 \times 10^{-22}$. Seria necessário $10^{21} \mathrm{~cm}^{3}$ de água para absorver um neutrino. Obviamente os dois concluiram que jamais poderia-se detectar neutrinos através deste decaimento inverso.

Em 1953, Fred Reines e Clyde Cowan, projetaram um experimento "que valesse uma vida", o projeto Poltergeist [10]. O objetivo deles era a detecção de um neutrino livre. O desenvolvimento de líquidos cintilantes tornou um sistema de detecção factível, quando operando em conjunto com um reator nuclear. O esquema final de detecção do neutrino nesse experimento está representado na 
Figura 1.2, e uma explicação mais detalhada do método se encontra no Capítulo 2. Em 1956 eles publicaram um artigo na revista Science denominado "Detection of the Free Neutrino: a Confirmation" que lhes rendeu o prêmio Nobel de 1995 [11]. Como Peierls comentou quase 50 anos após sua equívoca previsão, ele e Bethe não tinham imaginado a "existência de reatores nucleares produzindo vastas quantidades de neutrinos" e nem "a criatividade dos experimentalistas" $[9]$.

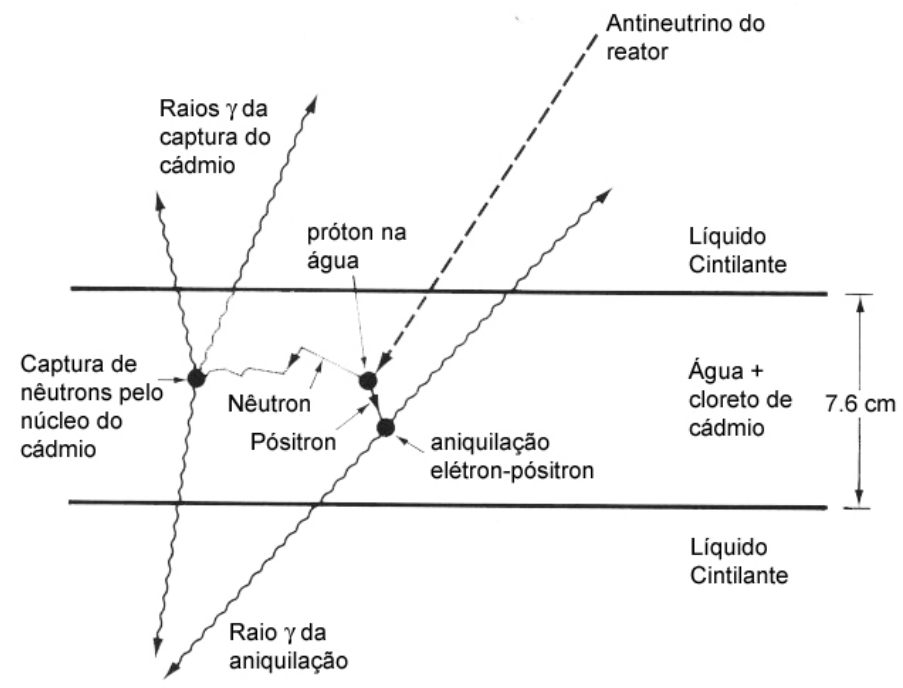

Figura 1.2: O método utilizado por Reines e Cowan era um processo composto por três etapas: primeiro um próton na água absorvia o neutrino, produzindo um par $e^{-} e^{+}$; então quase que imediatamente o pósitron se aniquilaria com um életron da água emitindo dois raios gamas em sentidos opostos, que seriam detectados pelo líquido cintilante; e finalmente, por volta de 5 microsegundos depois, o nêutron seria capturado pelo núcleo do cádmio, que então produziria mais raios gamas. A correlação temporal dos flashes de luz evidenciaria a deteç̧ão indireta do neutrino.

\subsection{Da teoria de Fermi à teoria Eletrofraca}

Foi Enrico Fermi o primeiro físico que conseguiu desenvolver uma teoria preditiva para os processos de decaimento utilizando a idéia de criação e destruição de partículas durante a interação [8]. Ironicamente seu trabalho foi rejeitado pela revista Nature por estar muito longe da realidade.

\subsubsection{Teoria de Fermi}

Na aproximação de Born desenvolvida para calcular processos de espalhamentos na Mecânica Quântica (MQ), a transição de um estado inicial $\psi_{i}$ para um estado 
final $\psi_{f}$ sob ação de um potencial $V(\vec{x})$ é dada pelo elemento de matriz

$$
M=\int d \vec{x} \psi_{f}^{*}(\vec{x}) V(\vec{x}) \psi_{i}(\vec{x}) .
$$

Este resultado pode ser generalizado para estados com duas partículas da seguinte maneira

$$
M=\int d \overrightarrow{x_{a}} d \overrightarrow{x_{b}} \psi_{f 1}^{*}\left(\vec{x}_{b}\right) \psi_{f 2}^{*}\left(\overrightarrow{x_{a}}\right) V\left(\overrightarrow{x_{a}}-\overrightarrow{x_{b}}\right) \psi_{i 1}\left(\overrightarrow{x_{b}}\right) \psi_{i 2}\left(\overrightarrow{x_{a}}\right)
$$

Fermi introduziu uma interação fenomenológica de alcance muito curto para obter um modelo consistente com os dados dos processos de decaimento:

$$
V\left(\overrightarrow{x_{a}}-\overrightarrow{x_{b}}\right)=G \delta\left(\overrightarrow{x_{a}}-\overrightarrow{x_{b}}\right)
$$

Com isso $M=G \int d \vec{x} \psi_{f 1}^{*}(\vec{x}) \psi_{f 2}^{*}(\vec{x}) \psi_{i 1}(\vec{x}) \psi_{i 2}(\vec{x})$. Porém duas outras modificações eram necessárias: primeiro as partículas do núcleo são descritas por espinores de Dirac pois são férmions; segundo, Fermi modelou a interação seguindo as forças eletromagnéticas, ou seja, por correntes vetoriais invariantes de Lorentz. Finalmente obteve à forma final de sua lagrangeana

$$
\mathcal{L}_{F}=\frac{G_{F}}{\sqrt{2}}\left(\overline{\Psi_{n}} \gamma^{\mu} \Psi_{p}\right)\left(\overline{\Psi_{e}} \gamma_{\mu} \Psi_{\nu}\right)
$$

Por este fato $G_{F}$ passou a ser chamada de constante de Fermi, e como seu valor é muito pequeno $\left(G_{F}=1,16637(1) \times 10^{-5} \mathrm{GeV}^{-2}[23]\right)$ a interação foi denominada de força fraca.

Contudo, dois problemas básicos foram assinalados no modelo de Fermi. A lagrangeana com quatro férmions acarreta violação da unitariedade $\left(\sigma \sim G_{F}^{2} E_{c m}^{2}\right)$ e não é renormalizável (alguns infinitos não podem ser absorvidos).

\subsubsection{Violação da paridade e helicidade do neutrino}

Conciliado à estes dois problemas, outra surpresa da natureza estava à se revelar. Em 1956, Tsung-Dao Lee e Cheng-Ning Yang chegaram à uma notável conclusão quando estudavam alguns problemas relacionados à física dos raios cósmicos. Eles mostraram que podiam explicar várias interações se os decaimentos das partículas não respeitassem a simetria esquerda-direita, chamada de paridade [12].

Intuitivamente espera-se que as leis da física não mudem com a troca $\vec{x} \rightarrow$ $-\vec{x}$ (chamada de paridade). De fato, esta intuição levou Pauli à escrever uma carta ao seu amigo Victor Weisskopf onde ele dizia que gostaria de apostar uma 
grande quantidade de dinheiro à favor da conservação dessa simetria [13]. Afinal, os experimentos anteriores medindo a radiação $\beta$ nunca evidenciaram nenhuma violação da paridade.

Ao ouvir uma palestra de Yang sobre suas idéias, Abdus Salam viajou para Londres refletindo no porquê a natureza deveria violar a paridade. Em sua desconfortável viagem de avião, ele voltou à pergunta de seu doutorado sobre o porquê neutrinos teriam massa aparentemente nula, e pensou "a Natureza tinha a escolha entre uma teoria que é esteticamente satisfatória mas cuja simetria esquerda-direita é violada, com um neutrino que viaja exatamente à velocidade da luz; e uma teoria cuja paridade é preservada, porém o neutrino tem uma massa ínfima..." [14]. Isso porque neutrinos não massivos não podem ter ambas quiralidades como é comentado no Apêndice A. Salam foi desencorajado a trabalhar nessa idéia por Peierls e Pauli.

Em 1957 Chien-Shiung Wu e seus colaboradores perceberam que os resultados produzidos nos experimentos até então eram isotrópicos pois não havia nenhuma direção privilegiada nesses experimentos. Assim eles colocaram um campo magnético para alinhar os spins de cobalto-60 e logo perceberam que os elétrons provenientes do decaimento ${ }^{60} \mathrm{Co} \rightarrow{ }^{60} \mathrm{Ni} e^{-} \overline{\nu_{e}}$, não saiam mais em todas as direções [15]. Ao inverter a direção do campo, o mesmo aconteceu com a direção da radiação. A conservação de paridade tinha sido abolida pela força fraca e o mundo dos físicos ficou perplexo. Quando esta notícia chegou a Pauli, ele escreveu novamente à Weisskopf dizendo-se acabado, e que agora podiam rir dele, porém pelo menos ele não tinha feito a aposta em dinheiro, à qual não poderia ter pago [13].

No ano seguinte, Maurice Goldhaber e seus colegas Lee Grodzins e Andrew Sunyar elaboraram um experimento visando medir a helicidade dos neutrinos [16]. A helicidade de uma partícula é a projeção de seu spin na direção de seu momento, se a partícula gira no sentido antihorário relativo à direção de seu momento, ela é dita negativa e vice-versa (veja Apêndice A). Como a medida direta do neutrino é complicada pelo seu alto poder de penetração, eles se utilizaram das conservações do momento linear e angular - conservação da helicidade - para obter o valor desejado. O esquema da experiência com europium-152 decaindo em samarium-152 excitado, liberando um fóton, está colocado na Figura 1.3. O fato de todos os fótons medidos terem helicidades negativas significou que realmente os neutrinos também o eram.

A Natureza parecia ter escolhido a violação da paridade e neutrinos sem massa de mão esquerda. 


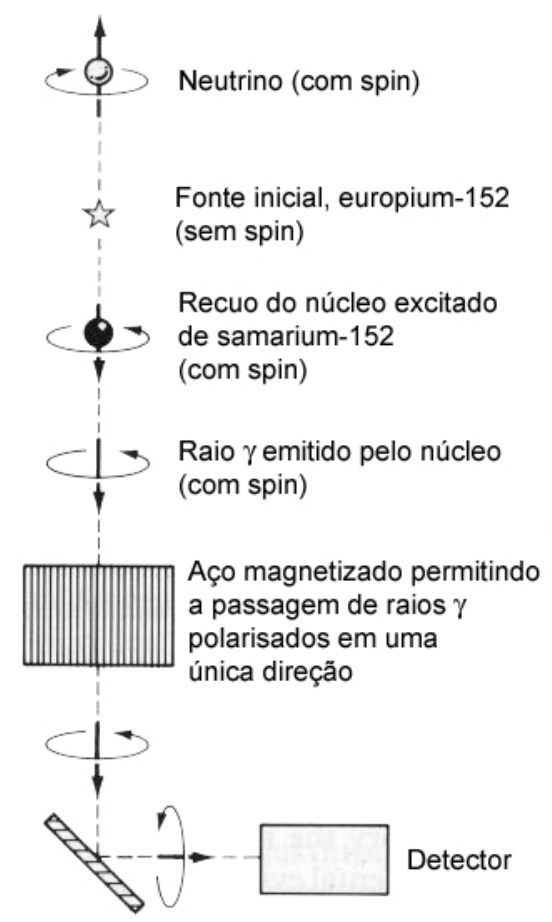

Figura 1.3: Esquema de determinação da helicidade do neutrino proposto por Goldhaber, Grodzins e Sunyar. O núcleo transmuta capturando um elétron e emitindo um neutrino; o núcleo e o neutrino se afastam em direções opostas conservando o momento angular total e linear.

\subsubsection{Teoria Eletrofraca}

Muitos trabalhos teóricos e experimentais foram feitos para se chegar na atual teoria da interação fraca. Na parte experimental vale destacar a observação de novas partículas como o muon $\mu$ e seu decaimento $\mu^{-} \rightarrow e^{-} \overline{\nu_{e}} \nu_{\mu}$, o píon $\pi$ e vários outros hádrons nos anos 50 .

$\mathrm{Na}$ parte teórica, a Eletrodinâmica Quântica (EDQ) foi desenvolvida por Feynman, Schwinger e Tomonaga em 1948 [17] dando um exemplo de teoria covariante e de calibre, com simetria abeliana e um bóson intermediando as interações, além de trazer à tona a questão de renormalização. Na tentativa de explicar a força fraca, foi proposto um modelo com apenas um bóson intermediário em 1957 por Schwinger, Lee e Yang, porém este ainda apresentava o problema da violação de unitariedade [18].

A teoria final unificando a força fraca e o eletromagnetismo foi elaborada durante a década de 60 por Glashow, Weinberg e Salam [19]. No entanto foi necessário usar a idéia de teorias não-abelianas e o mecanismo de Higgs para geração de massas. Além disso, a teoria somente foi reconhecida após o trabalho de 't Hooft de 1971 mostrando sua renormalizibilidade [181].

A teoria é baseada na quebra espontânea da simetria $S U(2)_{L} \times U(1)_{Y} \rightarrow$ 
$U(1)_{E M}$. Os índices fazem menção ao espaço onde atua a simetria.

Os bósons vetoriais intermediários, de spin 1, provém dos geradores dos grupos, portanto existem quatro nesta teoria. Após a quebra espontânea de simetria eles se misturam e dão origem as partículas: $W^{ \pm}, Z^{0}, \gamma$. A quebra faz com que as partículas fermiônicas e os bósons da interação fraca adquiram massa, deixando apenas o fóton sem massa, resultante da simetria residual $U(1)_{E M}$ da força eletromagnética.

Os férmions são colocados à mão. Existem questões teóricas como o cancelamento de anomalias para se acreditar que existam 3 famílias ${ }^{1}$, conjunto de partículas com os mesmos números quânticos, mudando apenas a escala de massa. Cada família é composta por dois léptons e dois quarks. As partículas de mão esquerda entram na teoria como dubletos de $S U(2)$ e de mão direita como singletos. Após a quebra espontânea de simetria, a carga elétrica de cada férmion é dada pela componente de isospin fraco mais a hipercarga pela fórmula: $Q=I+Y / 2$, e se mantém conservada pela simetria $U(1)_{E M}$. Para cada partícula fermiônica carregada está associada uma antipartícula de carga oposta.

Um ponto extremamente importante no MP que ainda não obteve confirmação experimental é a presença de uma partícula escalar, de spin 0, conhecida como bóson de Higgs. O campo de Higgs é responsável pela quebra espontânea de simetria do vácuo e pela geração de massas para todas as outras partículas através do mecanismo de Higgs [21]. Fisicamente, as partículas adquirem massa por sua interação com esse escalar, tanto maior o acoplamento, maior a sua massa. Por ser minimalista, o MP inclui apenas um desses escalares que já é suficiente para descrever os dados atuais. Entretanto, como só a experiência nos fornece a resposta final, sua existência ou não deve ser comprovada experimentalmente. Espera-se descobrir o bóson de Higgs no LHC.

Finalmente o MP também descreve a força forte incorporando a simetria $S U(3)_{C}$ agindo no espaço de cores, ou seja, atuando somente sobre os quarks. O MP é baseado na quebra $S U(3)_{C} \times S U(2)_{L} \times U(1)_{Y} \rightarrow S U(3)_{C} \times U(1)_{E M} . \mathrm{Um}$ resumo das partículas e interações do MP é apresentado na Tabela 1.1 e 1.2.

\subsection{Neutrinos no Modelo Padrão}

Os neutrinos são considerados partículas fundamentais sem massa, de carga nula e spin $\frac{1}{2}$ no MP. Desta forma são descritos pelos espinores de Weyl. Antineutrinos

\footnotetext{
${ }^{1}$ As famílias também são chamadas de gerações ou sabores.
} 


\begin{tabular}{|c|c|c|c|c|c|c|c|c|}
\hline & \multicolumn{3}{|c|}{ geração } & \multicolumn{5}{|c|}{ números quânticos } \\
\hline férmions & 1 & 2 & 3 & Y & I & Q & B & L \\
\hline \hline léptons de & $\nu_{e}$ & $\nu_{\mu}$ & $\nu_{\tau}$ & -1 & $\frac{1}{2}$ & 0 & 0 & 1 \\
mão esquerda & $e^{-}$ & $\mu^{-}$ & $\tau^{-}$ & -1 & $-\frac{1}{2}$ & -1 & 0 & 1 \\
\hline léptons de mão direita & $e^{-}$ & $\mu^{-}$ & $\tau^{-}$ & -2 & 0 & -1 & 0 & 1 \\
\hline quarks de & $u$ & $c$ & $t$ & $\frac{1}{3}$ & $\frac{1}{2}$ & $\frac{2}{3}$ & $\frac{1}{3}$ & 0 \\
mão esquerda & $d$ & $s$ & $b$ & $\frac{1}{3}$ & $-\frac{1}{2}$ & $-\frac{1}{3}$ & $\frac{1}{3}$ & 0 \\
\hline quarks de & $u$ & $c$ & $t$ & $\frac{4}{3}$ & 0 & $\frac{2}{3}$ & $\frac{1}{3}$ & 0 \\
mão direita & $d$ & $s$ & $b$ & $-\frac{2}{3}$ & 0 & $-\frac{1}{3}$ & $\frac{1}{3}$ & 0 \\
\hline \hline antiléptons de mão esquerda & $e^{+}$ & $\mu^{+}$ & $\tau^{+}$ & 2 & 0 & 1 & 0 & -1 \\
\hline antiléptons de & $\overline{\nu_{e}}$ & $\overline{\nu_{\mu}}$ & $\overline{\nu_{\tau}}$ & 1 & $-\frac{1}{2}$ & 0 & 0 & -1 \\
mão direita & $e^{+}$ & $\mu^{+}$ & $\tau^{+}$ & 1 & $\frac{1}{2}$ & 1 & 0 & -1 \\
\hline antiquarks de & $\bar{u}$ & $\bar{c}$ & $\bar{t}$ & $-\frac{1}{3}$ & $-\frac{1}{2}$ & $-\frac{2}{3}$ & $-\frac{1}{3}$ & 0 \\
mão esquerda & $\bar{d}$ & $\bar{s}$ & $\bar{b}$ & $-\frac{1}{3}$ & $\frac{1}{2}$ & $\frac{1}{3}$ & $-\frac{1}{3}$ & 0 \\
\hline antiquarks de & $\bar{u}$ & $\bar{c}$ & $\bar{t}$ & $-\frac{4}{3}$ & 0 & $-\frac{2}{3}$ & $-\frac{1}{3}$ & 0 \\
mão direita & $\bar{d}$ & $\bar{s}$ & $\bar{b}$ & $\frac{2}{3}$ & 0 & $\frac{1}{3}$ & $-\frac{1}{3}$ & 0 \\
\hline
\end{tabular}

Tabela 1.1: Os férmions do MP e seus números quânticos: $Y$ a hipercarga, $I$ a componente de isospin da força fraca, $Q$ a carga elétrica, $B$ o número bariônico e $L$ o número leptônico.

\begin{tabular}{|c|c|c|c|}
\hline Interações & Fraca & Eletromagnética & Forte \\
\hline \hline bósons & $W^{ \pm}, Z^{0}$ & $\gamma$ & 8 gluons \\
\hline$e, \mu, \tau$ & interage & interage & não interage \\
\hline neutrinos & interage & não interage & não interage \\
\hline quarks & interage & interage & interage \\
\hline $\mathrm{B}$ & conserva & conserva & conserva \\
\hline $\mathrm{L}$ & conserva & conserva & conserva \\
\hline paridade & não conserva & conserva & conserva \\
\hline conjugação de carga & não conserva & conserva & conserva \\
\hline sabor & não conserva & conserva & conserva \\
\hline
\end{tabular}

Tabela 1.2: As interações do MP, suas propriedades e conservação das simetrias.

são as partículas que acompanham o elétron no decaimento $\beta^{-}$e assim de mão direita, e neutrinos são aquelas que acompanham o pósitron no decaimento $\beta^{+}$ e portanto de mão esquerda.

Aqui estão colocados os principais resultados e propriedades dos neutrinos como entendidos no MP. Uma apresentação exaustiva dos mesmo pode ser encontrada na Ref. [22]. 


\subsubsection{Interações no Modelo Padrão}

Os neutrinos só participam da interação fraca, através da corrente carregada (CC) [19]

$$
\mathcal{L}^{C C}=\frac{g}{\sqrt{2}}\left(\begin{array}{lll}
\overline{\nu_{e}} & \overline{\nu_{\mu}} & \overline{\nu_{\tau}}
\end{array}\right) \gamma^{\lambda} \frac{1-\gamma_{5}}{2}\left(\begin{array}{c}
e \\
\mu \\
\tau
\end{array}\right) W_{\lambda}^{+}+\text {h.c. }
$$

e da corrente neutra $(\mathrm{NC})$

$$
\mathcal{L}^{N C}=\frac{g}{2 \cos \theta_{W}}\left(\begin{array}{lll}
\overline{\nu_{e}} & \overline{\nu_{\mu}} & \overline{\nu_{\tau}}
\end{array}\right) \gamma^{\lambda} \frac{1-\gamma_{5}}{2}\left(\begin{array}{c}
\nu_{e} \\
\nu_{\mu} \\
\nu_{\tau}
\end{array}\right) Z_{\lambda}^{0}+\text { h.c. } .
$$

\subsubsection{Número de famílias}

Existe uma boa razão experimental para se acreditar que só existam 3 famílias de férmions.

Ao se medir a largura de $Z^{0}$, considerando os decaimentos em todas as partículas mais leves que ela, existe um canal invisível que dá espaço à apenas 3 neutrinos com massa inferior à $m_{Z}=91,1876 \pm 0,0021 \mathrm{GeV}$ [23]. O experimento foi realizado no LEP, cujo resultado está colocado na Figura 1.4 [24].

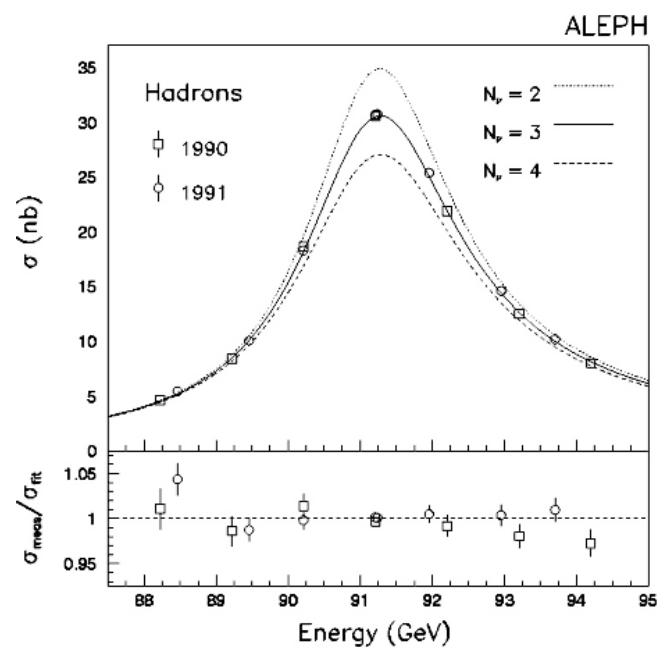

Figura 1.4: A combinação de resultados do LEP indica a existência de apenas 3 neutrinos com massa inferior à da partícula $Z^{0}$. 


\subsubsection{Massas e testes cinemáticos}

Existem limites nas massas dos neutrinos provenientes de vários tipos de experimentos, sendo os cosmológicos os mais restritivos [25]. Contudo, estes valores limites dependem muito do modelo e por isso é mais interessante elaborar experimentos que procurem pelas massas diretamente a partir da análise dos parâmetros cinemáticos dos produtos de um decaimento conhecido, onde aparecem os neutrinos.

Fermi foi o primeiro a propor um experimento assim no seu artigo de 1934 [8]. Ele mostrou que a massa do neutrino eletrônico poderia ser procurada através do decaimento $\beta$ do trítio,

$$
{ }^{3} \mathrm{H} \rightarrow{ }^{3} \mathrm{He}+e^{-}+\overline{\nu_{e}} .
$$

O espectro de energia dos elétrons emitidos seguem a seguinte expressão

$$
K(E) \propto(Q-E) \sqrt{\sqrt{(Q-E)^{2}-m_{\nu_{e}}^{2}}}
$$

denominada função de Kurie, cuja representação gráfica pode ser vista na Figura 1.5, onde $Q=m_{3_{H}}-m_{3} \mathrm{He}_{e}-m_{e} \simeq 18,6 \mathrm{keV}$ é a energia liberada no decaimento, $E$ é a energia do elétron e $m_{\nu_{e}}$ é a massa do neutrino do elétron. Pelo gráfico observa-se que somente se $m_{\nu_{e}} \neq 0$ a função se desviará de uma reta próximo ao ponto final do espectro.

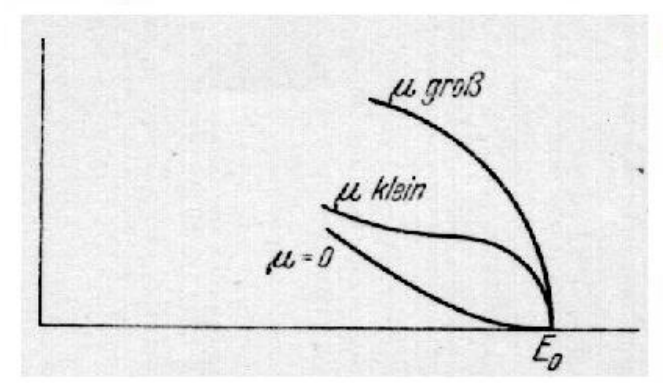

(a)

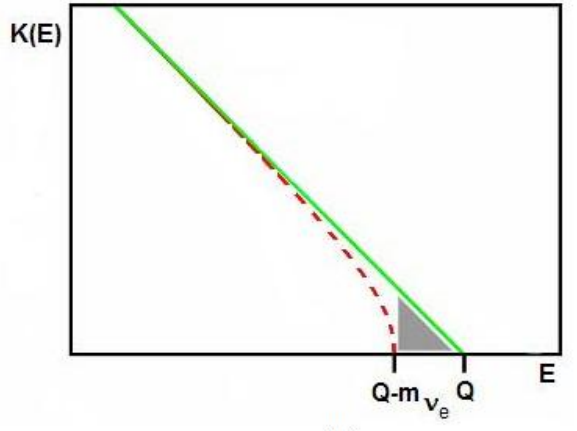

(b)

Figura 1.5: (a) Figura apresentada por Fermi [8] propondo o teste cinemático para detecção da massa do neutrino. (b) Gráfico esquemático da função de Kurie, as linhas tracejadas são para $m_{\nu_{e}} \neq 0$.

Até agora não se tem evidência em favor desse desvio, com um nível de 
confiança de $95 \%$ os limites atuais são [26, 27, 28]:

$$
\begin{aligned}
& m_{\nu_{e}}<2,2 \mathrm{eV} \\
& m_{\nu_{\mu}}<170 \mathrm{keV} \\
& m_{\nu_{\tau}}<15,5 \mathrm{MeV}
\end{aligned}
$$

os outros dois limites são obtidos estudando os decaimentos:

$$
\begin{aligned}
& \pi^{+} \rightarrow \mu^{+}+\nu_{\mu} \\
& \tau^{-} \rightarrow n \pi^{-}+\nu_{\tau}
\end{aligned}
$$

\subsubsection{Duplo decaimento $\beta$}

O decaimento $\beta$ ocorre pois o núcleo com um número atômico $Z$ tem energia de ligação menor que aquele com $Z+1$. Isso gera o processo de decaimento $(A, Z) \rightarrow(A, Z+1)+e^{-}+\bar{\nu}_{e}$. Entretanto, alguns núcleos com número atômico $Z+1$ apresentam energia de ligação menor que com $Z$, enquanto com $Z+2$ se apresentam mais estáveis que ambos. Juntamente com a lagrangeana eletrofraca, isso dá espaço para ocorrer o chamado duplo decaimento $\beta$ [29]:

$$
(A, Z) \rightarrow(A, Z+2)+2 e^{-}+2 \bar{\nu}_{e}
$$

Geralmente esse processo é denotado por $\beta \beta_{2 \nu}$ já que é acompanhado por dois antineutrinos. A amplitude é proporcional à $G_{F}^{2}$ e portanto ocorre muito raramente. De fato, o primeiro duplo decaimento $\beta$ foi observado no experimento de Elliot, Hahn e Moe com um núcleo de selenium [30]:

$$
{ }^{82} \mathrm{Se} \rightarrow{ }^{82} \mathrm{Kr}+2 e^{-}+2 \bar{\nu}_{e}
$$

com meia vida de $1,1 \times 10^{20}$ anos. Desde então, o processo foi observado em vários outros núcleos.

\subsection{Neutrinos além do Modelo Padrão}

Resultados recentes indicam a presença de massa não nula para neutrinos. Como o MP não admite neutrinos massivos, uma necessidade de construir e investigar nova física é colocada.

Esta seção se dedica à uma breve motivação à física de neutrinos além do MP, que será mais detalhada nos próximos capítulos com enfoque em oscilação de neutrinos. 


\subsubsection{Problema do neutrino solar}

Desde 1964, usando o resultado de Bahcall [31] da taxa de captura dos neutrinos provenientes do Sol através de estados excitados do ${ }^{37} \mathrm{Ar}$, Raymond Davis procurava, na mina de Homestake, observar alguns neutrinos por ano num imenso detector de 600 toneladas de solvente à base de cloro.

No final da década de 60 seus resultados foram surpreendentes [32], relataram um déficit no fluxo de neutrinos solares. Resultados que permanecem válidos até hoje.

Do ponto de vista astrofísico, somos tentados à pensar que este déficit poderia indicar alguma propriedade até então desconhecida do Sol. Muitas hipóteses e aproximações são consideradas nos modelos solares [33]. Assim, alguma das suposições poderia ser equívocas. Entretanto, outra hipótese sugerida já havia alguns anos, e que vinha sendo investigada, também poderia ser a solução, o fenômeno de oscilação de neutrinos.

\subsubsection{Oscilação de neutrinos}

Alguns anos antes, em 1958 Pontecorvo já havia introduzido a idéia de oscilação de neutrinos [34], que foi aperfeiçoada por Maki, Nakagawa e Sakata [35] em 1962. Esta idéia sugere que neutrinos de uma certa família podem se converter em neutrinos de outra durante a trajetória.

Vários experimentos com neutrinos de reatores já haviam sido realizados, contudo nenhum deles apresentava evidências de oscilação. Em 1969, Gribov e Pontecorvo interpretaram os resultados de Davis como uma evidência [36].

Apesar de sedutora, a análise dos resultados solares através da oscilação se mostrou de difícil aplicação. Finalmente, em 1985 Mikheev e Smirnov [37] retomaram o trabalho de Wolfenstein [38] cuja idéia era que as oscilações podiam ser ampliadas pela presença de matéria. Isso foi fundamental para que a teoria conseguisse se ajustar aos dados. No Capítulo 3 estudaremos com mais detalhes os resultados que evidenciam a oscilação, enquanto no Capítulo 4 apresentamos o formalismo teórico envolvido.

\subsubsection{Neutrinos massivos}

O mecanismo padrão para que haja oscilação é a introdução de massas, ainda que bem pequenas, para neutrinos.

Neste caso, uma física além do MP se faz necessária pois este não permite a geração de massa nem por correções radiativas ou efeitos não perturbativos. Além disso, a descrição dos neutrinos no MP por espinores de Weyl passa a ser 
incorreta e se cria uma necessidade de saber a origem das massas. Em particular se neutrinos são férmions de Dirac ou Majorana ([39], veja Apêndice B). Todas essas questões se abrem como janelas para termos acesso ao conhecimento da física além do MP. Torna-se portanto imprescindível a investigação de neutrinos.

Um estudo sobre os mecanismos de geração de massa, e suas consequências, no contexto da simetria $S U(2)_{L} \times U(1)_{Y}$ será discutido no Capítulo 5 .

\subsubsection{Duplo decaimento $\beta$ sem neutrinos}

Um processo que passaria a ser permitido com neutrinos massivos é o chamado duplo decaimento $\beta$ sem neutrinos, denotado por $\beta \beta_{\nu 0}$. A reação pode ser descrita como

$$
(A, Z) \rightarrow(A, Z+2)+2 e^{-}
$$

em que o neutrino emitido por um dos nucleons é absorvido pelo outro. É interessante observar que tal processo viola o número leptônico por duas unidades e a conservação da quiralidade de partículas sem massa (veja Apêndice A). Isso implica que neste caso os neutrinos devem ser de Majorana.

A amplitude da reação é proporcional à massa, contudo nenhum $\beta \beta_{\nu 0}$ foi observado até o momento.

\subsection{Cronograma histórico}

\begin{tabular}{|l|l|}
\hline 1896 & $\begin{array}{l}* 2 \text { Becquerel: evidência de efeitos radioativos espontâneos no } \\
\text { decaimento do urânio [2]. }\end{array}$ \\
\hline 1897 & $*$ Thomson: descoberta do elétron. \\
\hline 1911 & Rutherford: evidência do núcleo atômico. \\
\hline 1913 & $*$ Bohr: teoria quântica do espectro atômico. \\
\hline 1914 & Chadwick: primeira observação de que o espectro $\beta$ é contínuo [3]. \\
\hline 1927 & Ellis e Wooster: confirmação de que o espectro $\beta$ é contínuo [4]. \\
\hline 1930 & $\begin{array}{l}\text { Pauli: primeira proposta, através de uma carta, da existência de } \\
\text { uma partícula neutra, leve e interagindo fracamente [6]. }\end{array}$ \\
\hline 1932 & $*$ Chadwick: descoberta do neutron [40]. \\
\hline 1934 & $\begin{array}{l}\text { Pauli: explicação da continuidade do espectro } \beta, \text { proposta do } \\
\text { neutrino [7]. }\end{array}$ \\
\hline 1934 & Fermi: primeiro modelo da interação fraca [8]. \\
\hline 1937 & Neddermeyer e Anderson: primeira evidência do múon. \\
\hline
\end{tabular}

\footnotetext{
${ }^{2} \mathrm{O}$ asterisco * indica que o trabalho teve algum dos autores laureado pelo prêmio Nobel.
} 


\begin{tabular}{|c|c|}
\hline 1937 & Majorana: teoria do neutrino de Majorana [39]. \\
\hline 1946 & $\begin{array}{l}\text { Pontecorvo: proposta da detecção de neutrinos pela reação } \\
\overline{\nu_{e}}+{ }^{37} \mathrm{Cl} \rightarrow e^{-}+{ }^{37} \mathrm{Ar}[41] .\end{array}$ \\
\hline 1946 & Sakata e Inouë: previsão de $\nu_{\mu}[42]$. \\
\hline 1947 & $\begin{array}{l}\text { Pontecorvo: primeira idéia da universalidade da força fraca, ou } \\
\text { seja, os processos de captura e decaimento têm a mesma origem } \\
\text { [43]. }\end{array}$ \\
\hline 1948 & $\begin{array}{l}\text { * Feynman, Schwinger e Tomonaga: criação da teoria covariante } \\
\text { da EDQ [17]. }\end{array}$ \\
\hline Déc. 50 & Descoberta de várias partículas: $\Delta, \pi, \Sigma, \Lambda$.. \\
\hline 1956 & Reines e Cowan: observação do neutrino do elétron [10]. \\
\hline 1956 & * Lee e Yang: proposta de teste da conservação da paridade [12]. \\
\hline 1957 & Wu et al. : primeira observação da violação de paridade [15]. \\
\hline 1957 & $\begin{array}{l}\text { Salam; Lee e Yang; Landau: teoria do neutrino com duas } \\
\text { componentes [44]. }\end{array}$ \\
\hline 1957 & $\begin{array}{l}\text { Schwinger; Lee e Yang: teoria do bóson vetor intermediário (IVB) } \\
\text { para explicação da força fraca [18]. }\end{array}$ \\
\hline 1958 & $\begin{array}{l}\text { Goldhaber, Grodzins e Sunyar: primeira evidência para a } \\
\text { negatividade da helicidade do neutrino [16]. }\end{array}$ \\
\hline 1958 & Pontecorvo: introdução da idéia de oscilação de neutrinos [34]. \\
\hline 1961 & $\begin{array}{l}\text { * Glashow: introdução de um bóson neutro intermediando a força } \\
\text { fraca }\left(Z^{0}\right)[19] .\end{array}$ \\
\hline 1962 & * Lederman, Schwartz e Steinberg: observação do $\nu_{\mu}$ [45]. \\
\hline 1962 & $\begin{array}{l}\text { Maki, Nakagawa e Sakata: idéia da mistura e oscilação de } \\
\text { neutrinos [35]. }\end{array}$ \\
\hline 1964 & $\begin{array}{l}\text { Higgs; Englert e Brout; Guralnik, Hagen e Kibble: exemplo de } \\
\text { uma teoria de campo com quebra espontânea de simetria, sem } \\
\text { bósons de Goldstone sem massa, e um bóson vetorial massivo. } \\
{[21,46]}\end{array}$ \\
\hline 1964 & $\begin{array}{l}\text { * Salam e Ward: lagrangeana da teoria eletrofraca, estimativa da } \\
\text { massa do } W[19] \text {. }\end{array}$ \\
\hline 1964 & $\begin{array}{l}\text { * Gell-Mann; Zweig: introdução dos quarks como componentes } \\
\text { fundamentais dos hádrons. }\end{array}$ \\
\hline 1964 & Davis: proposta da pesquisa de neutrinos solares [47]. \\
\hline 1967 & $\begin{array}{l}\text { * Weinberg: síntese da lagrangeana da interação fraca e estimação } \\
\text { da massa de } Z \text { e } W[19] \text {. }\end{array}$ \\
\hline
\end{tabular}




\begin{tabular}{|c|l|}
\hline 1968 & $\begin{array}{l}* \text { Davis: resultado evidenciando déficit de neutrinos solares [32]. } \\
\text { Nascimento da astrofísica de neutrinos. }\end{array}$ \\
\hline 1968 & $*$ Salam: sínteses da lagrangeana da teoria eletrofraca [19]. \\
\hline 1969 & $\begin{array}{l}\text { Gribov e Pontecorvo: interpretação do déficit de neutrinos solares } \\
\text { como evidência de oscilação [36]. }\end{array}$ \\
\hline 1971 & $\begin{array}{l}* \text { 't Hooft: prova rigoroza da renormalização de uma teoria de } \\
\text { Yang-Mills com quebra espontânea de simetria [181]. }\end{array}$ \\
\hline 1975 & $*$ Perl et al. (SLAC): primeira deteç̧ão do $\tau^{-}$[48]. \\
\hline $1978 / 85$ & $\begin{array}{l}\text { Wolfenstein; Mikheev e Smirnov: introdução do efeito de matéria } \\
\text { em oscilação de neutrinos, denominado efeito MSW [37, 38]. }\end{array}$ \\
\hline 1987 & $\begin{array}{l}\text { Kamiokande: observação de neutrinos provenientes da supernova } \\
1987 A \text { [49]. }\end{array}$ \\
\hline 1989 & $\begin{array}{l}\text { MARK-II Colab.: primeira evidência que o número de neutrinos } \\
\text { leve seja 3 [50]. }\end{array}$ \\
\hline 1992 & $\begin{array}{l}\text { Colaborações do LEP: determinações precisas dos parâmetros de } \\
Z \text { [51]. }\end{array}$ \\
\hline 2001 & $\begin{array}{l}\text { Super-Kamiokande: fortes evidências de oscilação de neutrinos } \\
\text { atmosféricos [52]. }\end{array}$ \\
\hline 2001 & \begin{tabular}{l} 
SNO: confirmação de troca de sabor de neutrinos solares [53]. \\
\hline 1998 ormilab: experimento DONUT observa o neutrino do tau [54].
\end{tabular} \\
\hline
\end{tabular}




\section{Capítulo 2}

\section{Fontes de neutrinos e métodos de observação}

Calcula-se que nossos corpos humanos sejam atravessados por mais de 50 trilhões de neutrinos por segundo [55]. Esses neutrinos são provenientes de diversas fontes. Cinco fontes naturais e duas produzidas pelo homem são conhecidas, e estão apresentadas nas seções 2.1 e 2.2 respectivamente.

Apesar da vasta quantidade de neutrinos à nossa disposição, sua detecção ainda é complicada pelo alto poder de penetração destas partículas, que só interagem pela interação fraca. Desta forma, a seção 2.3 descreve as principais técnicas de deteç̧ão utilizadas nos experimentos de neutrinos. Os experimentos serão vistos no capítulo seguinte.

Uma seção final é consagrada à duas tabelas que resumem as informações discutidas no capítulo.

\section{$2.1 \quad$ Fontes naturais}

Cinco fontes naturais de neutrinos estão disponíveis hoje em dia. Entretanto, apenas neutrinos provenientes de três dessas podem ser observados regularmente em laboratórios: do Sol, da Terra e dos raios cósmicos.

Neutrinos de supernovas devem ter energias um pouco maiores que os solares, embora não se possa prever a explosão de uma supernova nas imediações de nossa galáxia.

Os neutrinos cósmicos de fundo possuem energia extremamente baixa, cerca de $10^{-10}$ vezes menor que os solares, e ainda não se conhece nenhuma maneira de se realizar sua detecção. 


\subsubsection{Neutrinos solares}

Os neutrinos solares são os neutrinos do elétron produzidos nas reações termonucleares que geram a energia do Sol.

A principal cadeia de reações em que o processo de fusão acontece no Sol é o ciclo $p p$, enquanto o ciclo carbono-nitrogênio-oxigênio (CNO) é responsável por menos de $2 \%$ da energia solar. Ambas cadeias resultam no processo de fusão de prótons em hélio-4: $4 p \rightarrow{ }^{4} \mathrm{He}+2 e^{+}+2 \nu_{e}+\gamma$, em que apenas $2,27 \%$ da energia é liberada na forma neutrinos (veja Fig. 2.1). A Tabela 2.1 apresenta um resumo da produção de neutrinos pelas diversas reações no Sol.

\begin{tabular}{llrc}
\hline Nome & Reação & $\left\langle E_{\nu}\right\rangle(\mathrm{MeV})$ & Fluxo $\left(\mathrm{cm}^{-2} \mathrm{~s}^{-1}\right)$ \\
\hline \hline pp & $p+p \rightarrow d+e^{+}+\nu_{e}$ & 0.2668 & $5,95 \times 10^{10}(1 \pm 0,01)$ \\
pep & $p+e^{-}+p \rightarrow D+\nu_{e}$ & 1.445 & $1,40 \times 10^{8}(1 \pm 0,015)$ \\
hep & ${ }^{3} \mathrm{He}+p \rightarrow{ }^{4} \mathrm{He}+e^{+}+\nu_{e}$ & 9.628 & $9,3 \times 10^{3}$ \\
${ }^{7} \mathrm{Be}$ & ${ }^{7} \mathrm{Be}+e^{-} \rightarrow{ }^{7} \mathrm{Li}+\nu_{e}$ & $(90 \%) 0.8631$ & $4,77 \times 10^{9}(1 \pm 0,10)$ \\
& & $(10 \%) 0.3855$ & \\
& & $6.735 \pm 0,036$ & $5,05 \times 10^{6}\left(1_{-0,16}^{+0,20}\right)$ \\
${ }^{8} \mathrm{~B}$ & ${ }^{8} \mathrm{~B} \rightarrow{ }^{8} \mathrm{Be} e^{*}+e^{+}+\nu_{e}$ & 0,7063 & $5,48 \times 10^{8}\left(1_{-0,17}^{+0,21}\right)$ \\
${ }^{13} \mathrm{~N}$ & ${ }^{13} \mathrm{~N} \rightarrow{ }^{13} \mathrm{C}+e^{+}+\nu_{e}$ & 0,9964 & $4,80 \times 10^{8}\left(1_{-0,19}^{+0,25}\right)$ \\
${ }^{15} \mathrm{O}$ & ${ }^{15} \mathrm{O} \rightarrow{ }^{15} \mathrm{~N}+e^{+}+\nu_{e}$ & 0,9977 & $5,63 \times 10^{6}(1 \pm 0,25)$ \\
${ }^{17} \mathrm{~F}$ & ${ }^{17} \mathrm{~F} \rightarrow{ }^{17} \mathrm{O}+e^{+}+\nu_{e}$ & & \\
\hline
\end{tabular}

Tabela 2.1: Reações de produção dos neutrinos solares, suas energias médias e fluxos de acordo com BP00 [56].
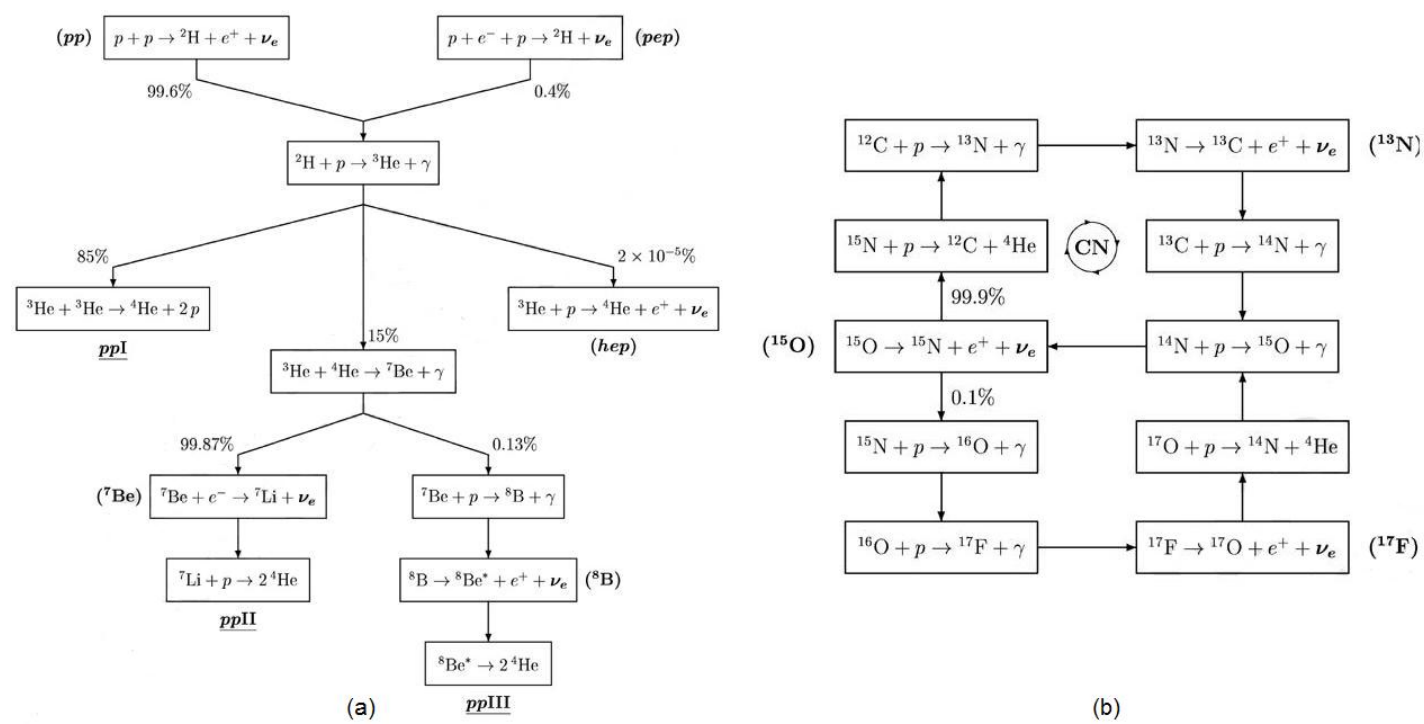

Figura 2.1: Ciclos (a) pp e (b) CNO no MSP BP00 [56].

Os fluxos de neutrinos e seus espectros energéticos são calculados no contexto de um Modelo Solar Padrão (MSP). Existem vários MSPs baseados em um 
conjunto de parâmetros observacionais: luminosidade superficial, idade, raio e massa solares; e em várias hipóteses básicas: simetria esférica, equilíbrio térmico e hidroestático, equação de estado de um gás ideal, e que apresenta abundância na superfície de vários elementos simliares à composição primordial [57]. Além da dependência no MSP escolhido, o cálculo do fluxo de neutrinos depende também de outros dados, como as seções de choque nucleares.

O fluxo dos neutrinos $p p$, mais abundantes, está relacionado com a luminosidade do Sol, que é bem conhecida, então possui uma incerteza pequena $\sim 1 \%$. Por outro lado, o fluxo dos neutrinos do ${ }^{8} B$ tem uma incerteza relativamente grande $\sim 20 \%$. O fluxo dos neutrinos mais energéticos provenientes da reação hep possuem uma grande incerteza, mas como este é bem pequeno, sua contribuição é praticamente desprezível. A Fig. 2.2 apresenta o resultado do fluxo de neutrinos solares em função da energia calculado no contexto do MSP de (a) Bahcall-Serenelli (BS05 [58]) e (b) Bahcall-Pinsonneault (BP00). Estes MSPs foram escolhidos por ser (a) o mais recente e (b) utilizado nas análises das revisões apresentadas no Capítulo 4, observe que a diferença não é apreciável.

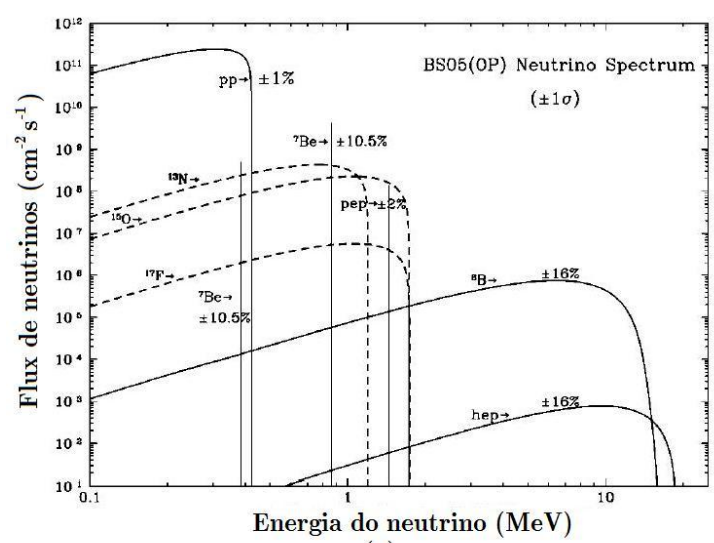

(a)

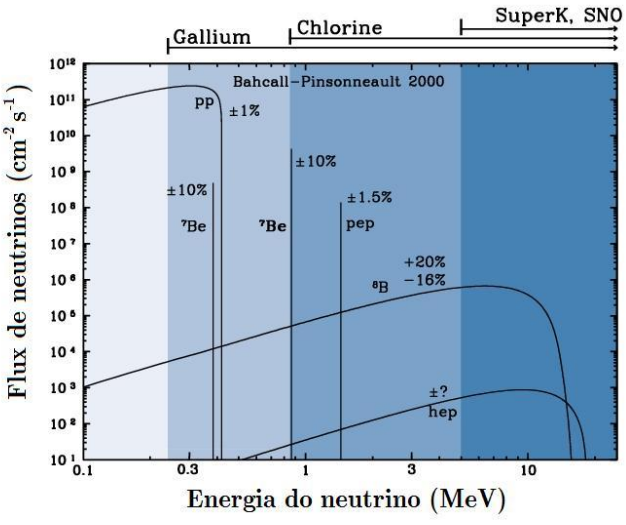

(b)

Figura 2.2: Fluxo de neutrinos solares em função da energia calculados de acordo com (a) BS05 [58] e (b) BP00 [56].

Outro parâmetro que pode ser estudado é a chamada assimetria Noite-Dia, que fornece a diferença de neutrinos que chegam na Terra entre o período noturno e diurno. A expectativa é

$$
A_{N-D}=2 \frac{N-D}{D+N}=0,
$$

pois não há motivos no MP para haver um fluxo maior de neutrinos solares noturnos $(N)$ do que diurnos $(D)$. 


\subsubsection{Neutrinos atmosféricos}

Neutrinos atmosféricos são aqueles resultantes da interação dos raios cósmicos com os núcleos atômicos na atmosfera terrestre, criando chuveiros de partículas, muitas das quais são instáveis e produzem neutrinos quando decaem (veja Fig. $2.3)$.

O principal mecanismo de produção de neutrinos atmosféricos é dado pela seguinte cadeia de reações:

$$
\begin{aligned}
p(\alpha, \ldots)+A r \rightarrow \pi^{ \pm}, K^{ \pm}, K^{0}, \ldots & \\
\pi^{ \pm}, K^{ \pm} \rightarrow & \mu^{ \pm}+\nu_{\mu}\left(\bar{\nu}_{\mu}\right) \\
& \mu^{ \pm} \rightarrow e^{ \pm}+\nu_{e}\left(\bar{\nu}_{e}\right)+\bar{\nu}_{\mu}\left(\nu_{\mu}\right) .
\end{aligned}
$$

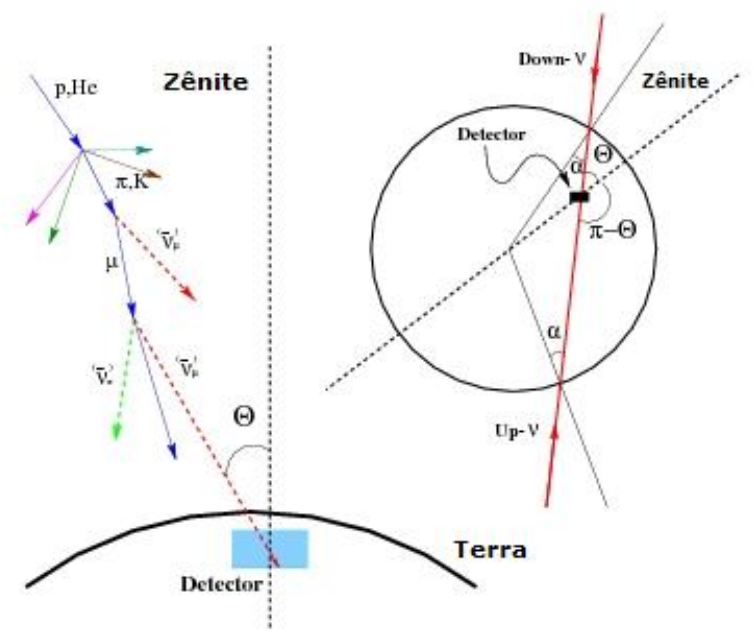

Figura 2.3: Esquema de detecção de neutrinos atmosféricos na Terra.

O fluxo de neutrinos pode ser calculado por uma técnica de Monte Carlo (MC) em função da energia (veja Fig. 2.4 (a)) ou do ângulo zênite (veja Fig. 2.4 (b)). Os principais parâmetros considerados no cálculo do fluxo são: espectro dos raios cósmicos, efeitos geomagnéticos e produção de neutrinos pelas interações hadrônicas $[59,60]$.

As principais características resultantes na dependência energética são:

- Ajuste: para $E_{\nu} \gtrsim 1 \mathrm{GeV}$, os fluxos obedecem aproximadamente uma lei de potência $d \Phi / d E \propto E^{-\gamma}$ onde $\gamma \sim 3(3.5)$ para os neutrinos e antineutrinos do múon (elétron). Para $E \lesssim 1 \mathrm{GeV}$, a dependência em energia é mais fraca como consequência da queda no espectro de raios cósmicos por efeitos geomagnéticos e modulações solares, o que pode ser vist na Fig. 2.5;

- Proporção $\bar{\nu} / \nu$ : para $E_{\nu} \lesssim 1 \mathrm{GeV}$, quando todos os píons e múons subseqüentes decaem antes de chegar na Terra, a proporção $\bar{\nu}_{e} / \nu_{e}=\pi^{-} / \pi^{+}<1$, 


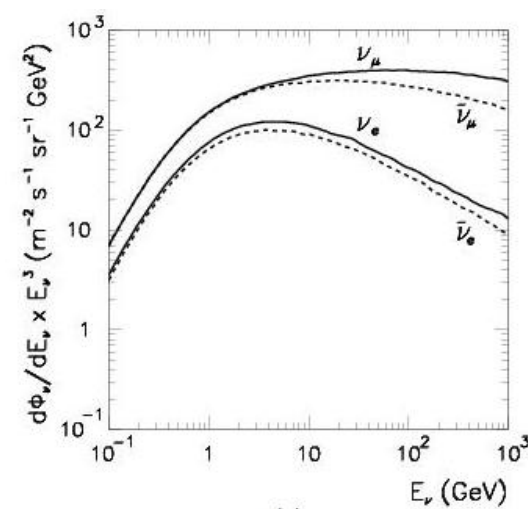

(a)

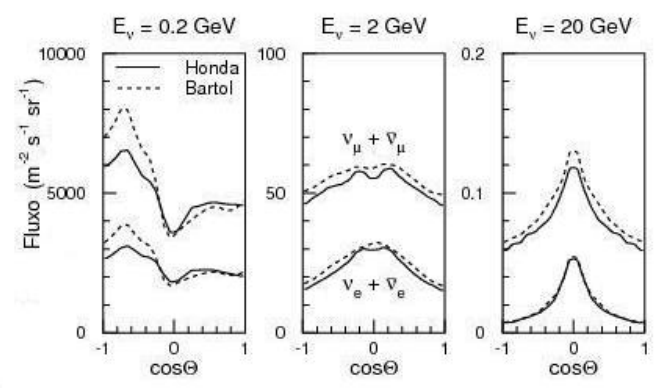

(b)

Figura 2.4: Dependência (a) energética e (b) com o ângulo zenital do fluxo dos neutrinos atmosféricos, calculadas pelo grupo de Bartol [60] para a mina de Kamioka onde se localiza o experimento de Super-Kamikande no Japão.

pois nestas energias os raios cósmicos são essencialmente prótons que produzem em média mais $\pi^{+}$do que $\pi^{-}$em suas interações. Em contrapartida, como $\pi^{+}\left(\mu^{+}\right)$produz $\nu_{\mu}\left(\bar{\nu}_{\mu}\right)$ em seu decaimento, espera-se $\bar{\nu}_{\mu} / \nu_{\mu}=1$. Quando a energia aumenta, os $\mu$ secundários não têm tempo de decair e $\bar{\nu}_{\mu} / \nu_{\mu}$ diminui;

- Proporção $\left(\nu_{\mu}+\bar{\nu}_{\mu}\right) /\left(\nu_{e}+\bar{\nu}_{e}\right)$ : para $E_{\nu} \lesssim 1 \mathrm{GeV}$, a proporção é bem próxima de 2 como esperado ingenuamente pela cadeia da Eq. (2.2). A energias mais altas, a proporção aumenta pois os $\mu$ não têm tempo de decair antes de alcançar a Terra e menos $\nu_{e}$ e $\bar{\nu}_{e}$ são produzidos.

E na dependência angular:

- Assimetria U-D ${ }^{1}$ : se $E$ é inferior à alguns GeV, os fluxos provenientes de baixo $\cos \Theta<0$, e de cima $\cos \Theta>0$, são assimétricos devido à efeitos geomagnéticos. Tais efeitos já são bem pequenos a $E=2 \mathrm{GeV}$ e o fluxo se torna U-D simétrico acima desta energia;

- Proporção horizontal-vertical: acima de alguns $\mathrm{GeV}$, os fluxos de neutrinos são máximos quando chegam horizontalmente e mínimos verticalmente. Isto se deve à uma diferença na densidade atmosférica que determina se os $\pi$ decaem antes de interagir novamente com o ar. Píons chegando horizontalmente viajam mais tempo em atmosfera menos densa e são mais propícios à decairem.

\footnotetext{
${ }^{1} \mathrm{U}$-D é um nome criado pelo autor que se utiliza das palavras em inglês $U p$, designando os neutrinos na direção para cima, e Down, para os neutrinos que vão para baixo.
} 


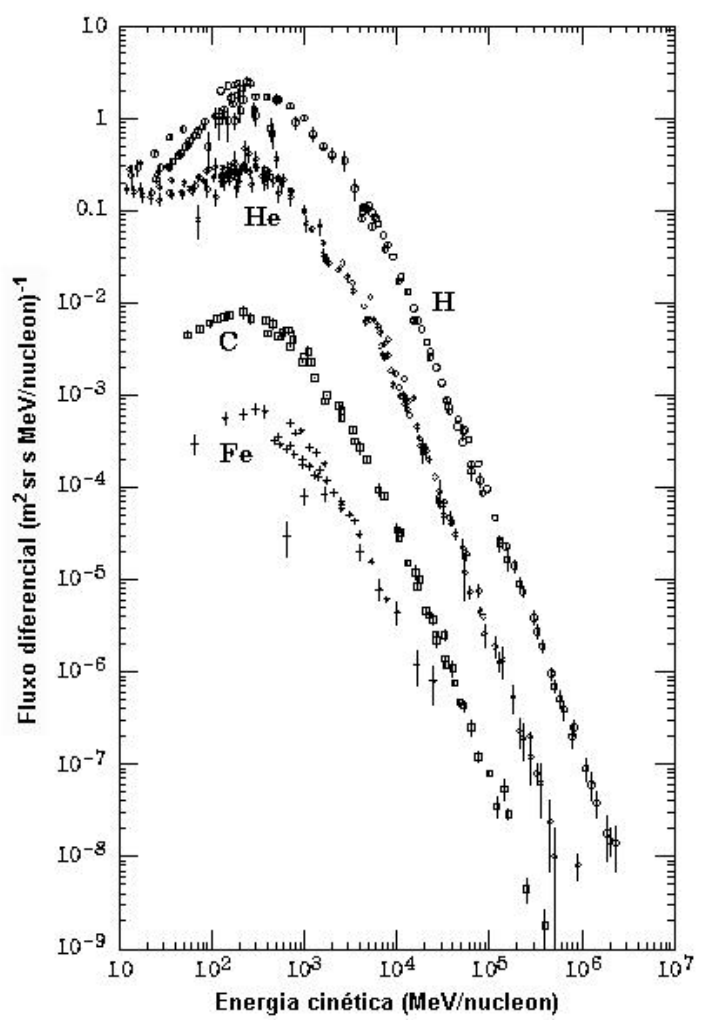

Figura 2.5: Os principais constituintes primários do espectro de raios cósmicos [61].

Os fluxos absolutos de neutrinos previstos teoricamente possuem uma incerteza de $20 \%$. Entretanto, a proporção

$$
R_{\mu / e}=\left(\nu_{\mu}+\bar{\nu}_{\mu}\right) /\left(\nu_{e}+\bar{\nu}_{e}\right)
$$

é menos sensível às incertezas dos fluxos absolutos e estima-se que os cálculos dessa razão tenham uma incerteza menor que $\sim 5 \%$. Outra proporção com boa previsão teórica é da simetria U-D:

$$
A_{U-D}=\frac{\text { Fluxo Up }(-1.0<\cos \Theta<-0.2)}{\text { Fluxo Down }(0.2<\cos \Theta<1.0)}=1
$$

para neutrinos do múon com energia acima de $1 \mathrm{GeV}$ aproximadamente.

\subsubsection{Neutrinos de supernovas}

Antes de se tornarem buracos negros ou estrelas de nêutrons, no final de sua evolução as estrelas com muita massa (acima de 10 vezes a massa do Sol) passam por um processo de explosão conhecida como supernova [62]. As supernovas são fontes valiosas de neutrinos pois $99 \%$ de sua energia é liberada através deles. A grande desvantagem das supernovas como fonte de neutrinos é a frequëncia de 
explosões, além de nunca termos previsão do instante exato em que ocorrerão, costuma-se ter de 1 à 3 supernovas por século na nossa galáxia.

Existem duas etapas que contribuem para a produção de neutrinos numa supernova. O primeiro ocorre após alguns milisegundos do colapso estelar, quando elétrons são absorvidos por prótons no processo de decaimento $\beta$ inverso: $e^{-}+p \rightarrow n+\nu_{e}$, estes neutrinos são os mais energéticos. O segundo processo ocorre durante a continuação do colapso e produz todos os 3 sabores de neutrinos e antineutrinos, sendo composto por reações como $e^{-}+e^{+} \rightarrow \nu \bar{\nu}$, $n+p \rightarrow n+p+\nu+\bar{\nu}$, etc [22].

Os neutrinos de supernova possuem energias médias estimadas entre 15 e 20 $\mathrm{MeV}$. Apesar de existirem muitos motivos para se estudar neutrinos de supernova (propriedades como massa, hierarquia, momento magnético, etc), apenas uma até hoje teve neutrinos detectados em laboratórios, a já famosa SN1987A que explodiu dia 13 de fevereiro de 1987. Um total de 20 eventos foram registrados e isso trouxe contribuições para o progresso na compreensão de tal fenômeno. Contudo, alguns enigmas ainda persistem: até pouco tempo nenhuma simulação de supernova tinha conseguido obter com sucesso a explosão da mesma, hoje em dia alguns mecanismos que levam a tal objetivo estão sendo investigados [63]; o fluxo e espectro de neutrinos e antineutrinos ainda não são bem conhecidos.

\subsubsection{Geoneutrinos}

Os geoneutrinos são antineutrinos do elétron produzidos pelas cadeias de decaimento de núcleos no interior da Terra. Os mais abundantes isótopos radioativos terrestres podem ser classificados em três grupos: ${ }^{238} \mathrm{U},{ }^{232} \mathrm{Th}$ e ${ }^{40} \mathrm{~K}$. Outros isótopos já decairam ou tem contribuições desprezíveis.

As principais reações que produzem os geoneutrinos são [64]

$$
\begin{array}{rll}
{ }^{238} \mathrm{U} & \rightarrow{ }_{100 \%} & { }^{206} \mathrm{~Pb}+8^{4} \mathrm{He}+6 e^{-}+6 \bar{\nu}_{e}+51.7 \mathrm{MeV} \\
{ }^{232} \mathrm{Th} & \rightarrow{ }_{100 \%} & { }^{208} \mathrm{~Pb}+6^{4} \mathrm{He}+4 e^{-}+4 \bar{\nu}_{e}+42.6 \mathrm{MeV} \\
{ }^{40} \mathrm{~K} & \rightarrow_{89,28 \%}{ }^{40} \mathrm{Ca}+e^{-}+\bar{\nu}_{e}+1,311 \mathrm{MeV} \\
{ }^{40} \mathrm{~K}+e^{-} & \rightarrow_{10,72 \%}{ }^{40} \mathrm{Ar}+\nu_{e}+1,505 \mathrm{MeV}
\end{array}
$$

Portanto a quantidade de calor radiogênico gerado e a luminosidade de neutrinos estão relacionados diretamente.

Um cálculo considerando 82 decaimentos $\beta$ na cadeia do U e 70 na cadeia do Th produz o fluxo e espectro de antineutrinos representado na Fig. 2.6. 


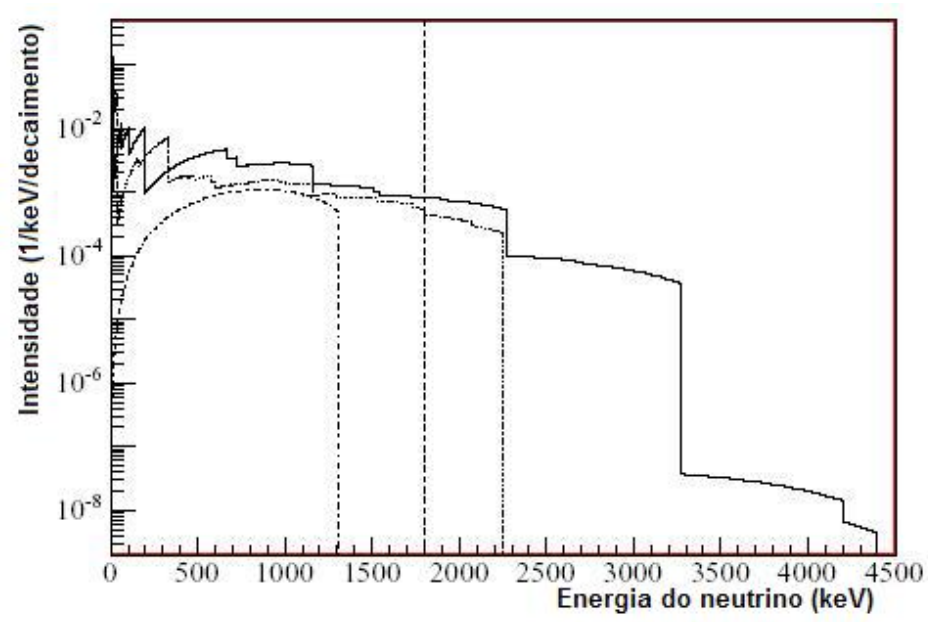

Figura 2.6: As distribuições de energia de $\bar{\nu}_{e}$ para as cadeias de decaimento do ${ }^{238} \mathrm{U}$ (linha), ${ }^{232} \mathrm{Th}$ (pontilhada) e ${ }^{40} \mathrm{~K}$ (linha e ponto). A linha tracejada na vertical representa o limiar para o experimento KamLAND [64].

\subsubsection{Neutrinos cosmológicos}

Assim como existe uma radiação cósmica de fundo composta por fótons, tudo indica que existe também neutrinos cósmicos de fundo relíquias do Big Bang. Estes neutrinos devem ter se desacoplados alguns segundos antes dos fótons e por isso têm uma temperatura abaixo da deles, aproximadamente $1,9 \mathrm{~K} \simeq 1,6 \times$ $10^{-4} \mathrm{eV}[22,65]$.

A medida destes neutrinos de fundo poderá impor muitas restrições às propriedades desta partícula além de investigar indiretamente os modelos cosmológicos vigentes. Entretanto, neutrinos são difíceis de se detectar, e por serem muito frios estes neutrinos de fundo podem nunca ser observados diretamente. Entretanto, existe uma probabilidade que neutrinos com energias extremamente altas, $\sim 10^{22}-10^{23} \mathrm{eV}$, possam interagir com estes neutrinos relíquias frios em pontos do espaço criando a partícula $Z$. Isto daria origem à rajadas dessas partículas que poderiam ser observadas na Terra, os Z-bursts. A observação destas rajadas poderia propiciar oportunidades de medidas como a escala de massa dos neutrinos, a energia dos neutrinos relíquias do Big Bang e observação de física na escala de teorias de unificação [66].

\subsection{Fontes humanas}

As duas fontes de neutrinos produzidas pelo homem são os aceleradores de partículas e os reatores nucleares.

Os aceleradores de partículas são laboratórios de física que provocam colisões 
à altas energias para o estudo de interações fundamentais. Sendo assim, criam neutrinos que costumam ter energias elevadas (dezenas de $\mathrm{GeV}$ ).

As usinas nucleares são construídas para geração de energia elétrica comercial, abastecendo casas, comércios e indústrias. Desta forma, os neutrinos produzidos nos reatores são frutos secundários das fissões nucleares e portanto possuem energias relativamente baixas (alguns $\mathrm{MeV}$ ).

Através da colocação de detectores de neutrinos próximos à aceleradores e reatores podemos verificar de fato a sua presença e medir seu fluxo. O fluxo de neutrinos nestes experimentos são mais previsíveis que nas fontes naturais por causa do controle humano de sua produção.

\subsubsection{Neutrinos de aceleradores}

Alguns aceleradores de partículas têm sido utilizados para produzir neutrinos. Existem vários aceleradores espalhados pelo mundo, e assim algumas características do feixe de neutrinos variam de acordo com o experimento. Aqui estão apresentadas as principais delas, comuns à maioria dos aceleradores [67].

A técnica tradicional é colidir prótons de alta energia com um alvo fixo criando mésons secundários, em particular, $\pi^{ \pm}$e $K^{ \pm}$. Estes mésons podem ser capturados e focalizados através de imãs, onde então decaem e produzem um feixe de neutrinos constituído predominantemente de $\nu_{\mu}$. A principal cadeia de reações é dada pela Eq. (2.2), com um alvo selecionado no lugar do ar, normalmente Be ou grafite.

As três principais componentes de um feixe convencional em um acelerador de altas energias são:

- o alvo em que os prótons colidem para produção de píons e káons;

- o sistema de focalização que guia os mésons na direção desejada dos feixes de neutrinos;

- o túnel de decaimento (geralmente evacuado) onde os mésons decaem e produzem neutrinos e múons.

Da cinemática do decaimento dos mésons, segue que um neutrino de massa nula possui a seguinte energia

$$
E=E_{\pi(K)} \frac{m_{\pi(K)}^{2}-m_{\mu}^{2}}{m_{\pi(K)}^{2}}
$$

onde $E_{\pi(K)}$ é a energia do méson primário. Enquanto que se fosse adicionada massa aos neutrinos, a primeira correção à essa energia seria $E_{1} \simeq E+\epsilon \frac{m_{\nu}^{2}}{2 E}$, 
$\operatorname{com} \epsilon=\frac{1}{2}\left(1-\frac{m_{\mu}^{2}}{m_{\pi(K)}^{2}}\right) \approx 0,2$, de onde vemos que experimentos em aceleradores poderiam, em princípio, ter sensibilidade à massa de neutrinos. Contudo, esse é o método apresentado no capítulo anterior, e a precisão atual só nos permite impor limites superiores à estas massas.

Existem três tipos de feixes de neutrinos convencionais: Feixes de Banda Larga (FBL), Feixes de Banda Estreita (FBE) e Feixes Fora do Eixo (FFE).

- Os FBL são caracterizados por um largo espectro de altas energias. FBL são os melhores feixes para descobertas. O empecilho é se o sinal vem de uma pequena região do espectro, já que este pode ser encoberto pelos ruídos induzidos pelos neutrinos fora desta região.

- Ao contrário, os FBE produzem neutrinos praticamente monocromáticos. Isto pode ser obtido selecionando uma pequena fatia do momento dos mésons primários $\pi$ e $K$. Entretanto, o fluxo do feixe se reduz significativamente. Este é um problema importante na procura por oscilações.

- Os FFE são os que melhor oferecem uma relação entre fluxo grande e estreita faixa de energia. Esta técnica envolve o desenvolvimento de um feixe que produz e focaliza uma banda larga de mésons numa certa direção (como no caso de FBL), porém os detectores são colocados num determinado ângulo em relação à direção do feixe. Como o decaimento do píon é de dois corpos, para um certo ângulo a energia do neutrino é quase independente da energia do píon.

As energias dos feixes de neutrinos dos experimentos variam de poucos $\mathrm{GeV}$ à poucas centenas de $\mathrm{GeV}$. É importante notar que independente do método utilizado, existem problemas comuns à todos os feixes de neutrinos convencionais:

- os hádrons utilizados levam à grandes incertezas proveninetes das dificuldades teóricas de se descrever processos hadrônicos. Isto traz dificuldades na previsão do fluxo de neutrinos e seu espectro com boa precisão;

- além do sabor muônico predominante do feixe $\left(\nu_{\mu}\right)$, existe uma pequena contaminação de outros sabores $\left(\bar{\nu}_{\mu}, \nu_{e}\right.$ e $\left.\bar{\nu}_{e}\right)$;

- devido ao fato dos neutrinos não poderem ser focalizados subseqüentemente, a grandes distâncias o fluxo de neutrino varia com $L^{-2}$.

Algumas destas limitações podem ser superadas utilizando-se novos conceitos de feixes como os feixes $\beta$ ou as Fábricas de Neutrinos [67, 68]. 


\subsubsection{Neutrinos de reatores}

Os reatores nucleares são as maiores fontes de neutrinos produzidos pelo homem. A produção de neutrinos em reatores ocorre pelo decaimento $\beta$ proveniente dos fragmentos fissionados em nêutrons nas usinas.

Os primeiros experimentos usavam reatores militares ou de pesquisa. Hoje em dia, com a necessidade de maiores fluxos (potências), estações geradoras de potências comerciais são utilizadas. Reatores modernos típicos têm potência térmica acima de $3 \mathrm{GW}$, correspondendo a aproximadamente $7,7 \times 10^{20} \bar{\nu}_{e} / \mathrm{s}$. Geralmente, nestas usinas nucleares, cada reator está localizado próximo um do outro de forma que o fluxo de neutrinos detectados é a soma das contribuições de cada núcleo.

A energia média do feixe de $\bar{\nu}_{e}$ produzido é $\sim 3 \mathrm{MeV}$, com valor máximo não ultrapassando $10 \mathrm{MeV}$.

Os quatro principais isótopos que contribuem para o fluxo e o espectro do antineutrino são: ${ }^{235} \mathrm{U},{ }^{238} \mathrm{U},{ }^{239} \mathrm{Pu}$ e ${ }^{241} \mathrm{Pu}$. Antineutrinos são produzidos através de longas cadeias de decaimento e diferentes tipos de decaimento $\beta$ têm que ser incluídos para explicar os observáveis experimentais. Tendo em vista a grande estatística de eventos, Vogel e Engel [69] encontraram uma parametrização fenomenológica do espectro que depende apenas dos isótopos envolvidos dada por:

$$
\left(\frac{d N_{\nu}}{d E}\right)_{j}=e^{a_{0 j}+a_{1 j} E+a_{2 j} E^{2}}, \quad j={ }^{235} \mathrm{U},{ }^{238} \mathrm{U},{ }^{239} \mathrm{Pu},{ }^{241} \mathrm{Pu},
$$

onde os valores ajustados dos parâmetros $a_{i j}$ são mostrados na Tabela 2.2. Este espectro é dado em unidades de $\bar{\nu}_{e} /(\mathrm{MeV}$-fissão) e depende basicamente da potência térmica, da composição isotópica do combustível do reator. Portanto, dado este espectro, resta determinar quantas fissões de cada isótopo ocorreram. Para cada reator, isto dependerá de três fatores: da potência térmica do reator; da composição isotópica do reator; e da quantidade de potência térmica emitida durante o fissionamento de um núcleo de um dado isótopo.

Assim que o reator começa a operar, a concentração de ${ }^{235} \mathrm{U}$ diminui e dos outros aumenta. Ao invés de levar em conta a evolução dos isótopos, podese assumir as frações típicas das fissões dos isótopos para os quatro materiais fissíveis de acordo com a Ref. [70] como mostra a Tabela 2.3.

Finalmente, notemos que a energia térmica associada ao fissionamento de cada núcleon, cujos valores relevantes se encontram na Tabela 2.3, é conhecida [71]. Portanto, o espectro emitido pelo $k$-ésimo reator do experimento pode ser 


\begin{tabular}{|c|c|c|c|c|}
\hline Isótopo & ${ }^{235} \mathrm{U}$ & ${ }^{239} \mathrm{Pu}$ & ${ }^{238} \mathrm{U}$ & ${ }^{241} \mathrm{Pu}$ \\
\hline \hline$a_{0}$ & 0,870 & 0,896 & 0,976 & 0,793 \\
\hline$a_{1}$ & $-0,160$ & $-0,239$ & $-0,162$ & $-0,080$ \\
\hline$a_{2}$ & $-0,0910$ & $-0,0981$ & $-0,0790$ & $-0,1085$ \\
\hline
\end{tabular}

Tabela 2.2: Parâmetros dos isótopos para o ajuste do espectro de neutrinos em unidades de $\bar{\nu}_{e} /(\mathrm{MeV}$-fissão) [69].

escrito como:

$$
S(k, E)=\sum_{j}\left(\frac{d N_{\nu}}{d E}\right)_{j} \int \frac{W(k, t)}{f(j) \xi(j)} d t \quad j={ }^{235} \mathrm{U},{ }^{238} \mathrm{U},{ }^{239} \mathrm{Pu},{ }^{241} \mathrm{Pu},
$$

sendo $f(j)$ e $\xi(j)$ o valor típico da fração e a energia de fissão para cada $j$ ésimo isótopo, respectivamente mostrado na Tabela 2.3 e, $W(k, t)$ é a potência do $k$-ésimo reator.

\begin{tabular}{|c|l|l|l|l|}
\hline & $\begin{array}{l}\text { Início do Ci- } \\
\text { clo(\%) }\end{array}$ & $\begin{array}{l}\text { Final do Ci- } \\
\text { clo(\%) }\end{array}$ & $\begin{array}{l}\text { Valor } \\
\text { Típico(\%) }\end{array}$ & $\begin{array}{l}\text { Energia por } \\
\text { Fissão }(\mathrm{MeV})\end{array}$ \\
\hline \hline${ }^{235} \mathrm{U}$ & 60,5 & 45,0 & 53,8 & 201,7 \\
\hline${ }^{239} \mathrm{Pu}$ & 27,2 & 38,8 & 32,8 & 205,0 \\
\hline${ }^{238} \mathrm{U}$ & 7,7 & 8,3 & 7,8 & 210,0 \\
\hline${ }^{241} \mathrm{Pu}$ & 4,6 & 7,9 & 5,6 & 201,7 \\
\hline
\end{tabular}

Tabela 2.3: A fração de fissões no começo e no final de cada ciclo e um valor típico extraídos da Ref. [70], e a energia por fissão [71] para os quatro isótopos fissíveis num reator nuclear.

Para exemplificar o fluxo de neutrinos de reatores, a Fig. 2.7 apresenta o fluxo calculado teoricamente para o experimento de KamLAND, onde 69 reatores estão envolvidos à uma distância média de aproximadamente $180 \mathrm{~km}$.

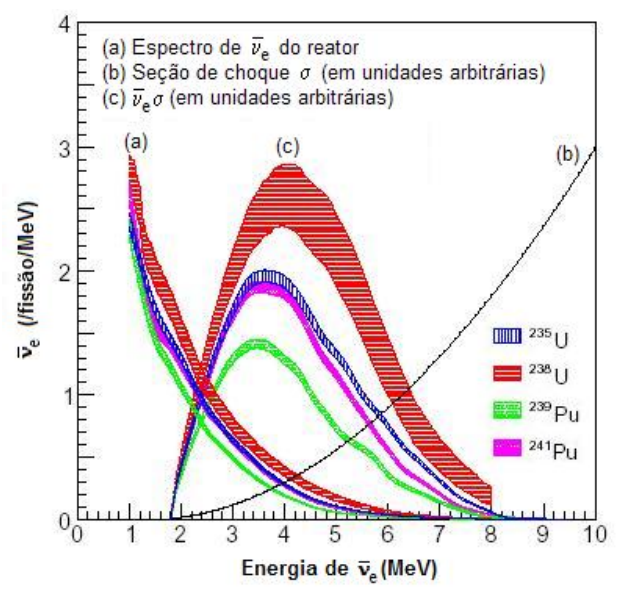

Figura 2.7: Fluxo de neutrinos em KamLAND (reatores) [72]. 


\subsection{Métodos de observação}

De um modo ou outro, todos os experimentos que procuram por neutrinos se utilizam dos métodos de detecção listados nesta seção. As diferenças que existem são adaptações ao local, ao financiamento ou outros fatores físico-econômicos de todo projeto. Obviamente, toda modificação visa aumentar a eficiência e a precisão, reduzindo evidentemente o custo.

Os neutrinos são detectados através da interação com algum alvo. Esta interação pode ser identificada por dois processos diferentes: pela excitação de um núcleo distinguindo-o dos outros (detecção radioquímica); ou pelo rastreamento das partículas secundárias. O segundo caso é o mais tradicional em física de partículas por fornecer mais informações sobre o neutrino incidente, e assim já existem várias técnicas de se identificar as partículas resultantes [73].

No setor de neutrinos, em particular, de troca de sabores, é importante conseguir distinguir os léptons nas interações de CC. No contexto, as técnicas mais utilizadas são de cintiladores líquidos e de radiação Cherenkov em água. Além destes, em laboratórios próximos à aceleradores também são encontrados calorímetros e outros tipos de câmaras de deteç̧ão. A última parte desta seção é consagrada aos calorímetros. Os outro tipos de câmaras, como as utilizadas em detectores de emulsão híbridos ou câmaras de projeção com argônio líquido, não são descritas aqui pois não são utilizados amplamente e requerem muito espaço para explicação, além de não trazerem grandes novidades em relação aos calorímetros $[67,73]$.

\subsubsection{Detecção radioquímica}

O método radioquímico é baseado na interação:

$$
\nu_{e}+X \rightarrow e^{-}+Y
$$

de um núcleo $X$ absorve um neutrino, convertendo-se em um isótopo radioativo de um outro elemento $Y$ com vida média de poucas semanas. Normalmente o detector é deixado em exposição por algumas semanas para absorver neutrinos. Após este período, o número de átomos do elemento final radioativo é contado e este valor fornece o fluxo de neutrinos [22].

Os núcleos que já foram usados em experimentos estão listados na Tabela 2.4, juntamente com o limiar de energia de cada experimento e a vida média do núcleo secundário. A unidade mais conveniente nestes experimentos é SNU (unidades de neutrinos solares), que representa o número de capturas ocorridas por segundo em $10^{36}$ átomos no detector. Outras possibilidades para o material 
do detector seriam: ${ }^{7} \mathrm{Li},{ }^{81} \mathrm{Br}$ e ${ }^{127} \mathrm{I}$; além de experimentos geoquímicos visando a abundância dos elementos: ${ }^{97} \mathrm{Mo},{ }^{98} \mathrm{Mo},{ }^{81} \mathrm{Br}$, etc. Entretanto, nenhum experimento empregando estas sugestões foi colocado em prática.

\begin{tabular}{|c|c|c|c|}
\hline \multicolumn{2}{|c|}{ Núcleos } & $E_{L}$ & $\tau$ \\
$X$ & $Y$ & $(\mathrm{MeV})$ & $($ dias $)$ \\
\hline${ }^{37} \mathrm{Cl}$ & ${ }^{37} \mathrm{Ar}$ & 0,814 & 35 \\
\hline${ }^{71} \mathrm{Ar}$ & ${ }^{71} \mathrm{Ge}$ & 0,233 & 11,4 \\
\hline
\end{tabular}

Tabela 2.4: Reações empregadas para detecção radioquímica de neutrinos eletrônicos. Todas as reações são da forma $\nu_{e}+X \rightarrow e^{-}+Y$ para núcleos $X$ e $Y$ apropriados.

A principal vantagem do método radioquímico é o baixo limiar de energia dos neutrinos detectados. No caso do Gálio é 0, $233 \mathrm{MeV}$, permitindo que neutrinos de várias cadeias solares possam ser observados. As desvantagens do método é que não se pode determinar a energia do neutrino, nem sua direção e nem o instante de sua detecção.

\subsubsection{Radiação Cherenkov}

Vários materiais podem ser utilizados nos experimentos baseados na técnica de radiação Cherenkov. Contudo, no contexto de neutrinos é necessário colocar um grande volume de material em exposição, o que requer um custo baixo do material empregado. Portanto a água se apresenta como melhor solução. O neutrino interage com os elétrons dos átomos de hidrogênio e oxigênio através do espalhamento elástico:

$$
\nu+e^{-} \rightarrow \nu+e^{-}
$$

No final, o elétron adquire energia proveniente do neutrino. Se esta energia for suficientemente grande, o elétron passará a se mover mais rápido que a velocidade efetiva da luz na água. Neste caso, o elétron emitirá uma radiação conhecida como radiação Cherenkov² [73].

Vários tubos ou células fotomultiplicadoras ${ }^{3}$ (TFM) são colocados em volta do experimento para amplificação deste sinal. A observação desta radiação constitui uma medida indireta de um neutrino.

As imagens coletadas tem forma de anéis já que a radiação emitida pela partícula forma um cone de luz. Dependendo da atividade eletromagnética deste anel é possível distinguir $e^{-}$de $\mu^{-}$. A Fig. 2.8 mostra essa diferença [74].

Existem várias vantagens em se aplicar esta técnica. Em primeiro lugar, a radiação Cherenkov possui uma distribuição angular bem concentrada dentro de

\footnotetext{
${ }^{2}$ Essa radiação é geralmente compreendida fazendo uma analogia ao efeito supersônico e quebra da barreira do som.

${ }^{3}$ Os TFMs são dispositivos eletrônicos que transformam sinal luminoso em impulso elétrico.
} 


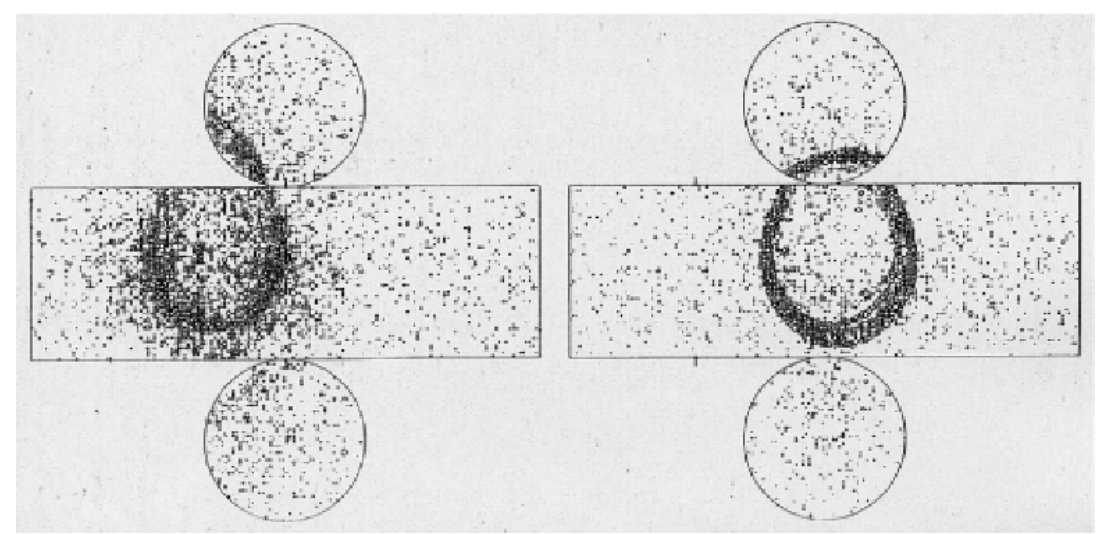

Figura 2.8: Imagens dos anéis de Cherenkov produzidos no detector Super-Kamiokande por (a) um elétron e (b) um múon.

um ângulo $\theta \sim \sqrt{m_{e} / E}$, desta forma pode-se inferir a direção proveniente do neutrino. Além disso, a deteç̧ão é feita em tempo real. Neutrinos dos sabores $\nu_{\mu}$ e $\nu_{\tau}$ são detectados com uma eficiência aproximadamente 6 vezes menor pois as seções de choque são diferentes (não possuem a contribuição de CC).

A grande desvantagem deste método é a energia limiar. Para reduzir o ruído proveniente de outras fontes, o corte de energia imposto aos neutrinos costuma ser elevado em relação ao método radioquímico, da ordem de $5 \mathrm{MeV}$.

\section{Detecção com água pesada}

Água pesada é constituída de um núcleo de deutério. A presença deste elemento permite a detecção pela radiação Cherenkov por três canais diferentes:

1. Pela corrente carregada (CC) $\nu_{e}+d \rightarrow p+p+e^{-}$, com $E_{L}=1,44 \mathrm{MeV}$, medindo apenas o fluxo de $\nu_{e}$;

2. Pela corrente neutra (NC) $\nu+d \rightarrow \nu+n+p$, com $E_{L}=2,23 \mathrm{MeV}$, detectando todos os sabores de neutrinos com mesma seção de choque pois não há participação de nenhum lépton carregado. A detecção do nêutron confirma este processo.

3. Pelo espalhamento elástico (ES) $\nu+e^{-} \rightarrow \nu+e^{-}$, que tem seções de choque diferentes para os diferentes sabores de neutrinos.

\subsubsection{Contagem em líquido cintilante}

A contagem em líquido cintilante é uma técnica já padrão de se fazer medidas da radiação $\beta$. 
O fluido cintilador é chamado de "cocktail", composto de três produtos químicos: solvente, emulsificador e fluor ${ }^{4}$. As amostras que sofrem o decaimento ficam suspensas neste fluido. Para garantir eficiência na transferência de energia entre as partículas $\beta$ emitidas e a solução, esta costuma ser um solvente do material radioativo. Parte da energia absorvida pelo solvente excita suas moléculas (outra parte é transformada em calor e ionização). A desexcitação libera raios $\gamma$ ultravioletas que são transformados em flashes de luz visível (próxima ao azul) pelo fluor. O número total de fótons provenientes das moléculas de fluor excitadas constituem a cintilação. A intensidade da luz é proporcional à energia inicial da partícula $\beta$. Novamente, os TFMs são colocados em volta do detector e transformam o sinal captado em impulsos elétricos.

Existem pelo menos três formas de se utilizar um líquido cintilante para realização de medidas:

1. Para contagem da radiação $\beta$, como descrito acima, em que as amostras do material radioativo ficam em suspensão no líquido e TFMs são colocados em volta do tanque para armazenamento dos sinais.

2. Na deteç̧ão de partículas, em que no lugar das amostras radiativas é colocado um material para reagir com as partículas procuradas liberando elétrons ou pósitrons, simulando uma radiação $\beta$. No caso de neutrinos, a reação mais utilizada é o decaimento $\beta$ inverso: $\bar{\nu}_{e} p \rightarrow e^{+} n$. Assim, o líquido cintilante de uma amostra deve ser constituído com alta densidade de prótons.

3. Para contagem de fótons, tendo uma função parecida com os TFMs. Neste caso, o cintilador converte a excitação de um material transparente causada por uma partícula carregada em luz visível. No experimento de Reines e Cowan, comentado no primeiro capítulo, a absorção de neutrinos ocorria num meio com água e cádmio, contudo os raios gamas liberados pela aniquilação $e^{-} e^{+}$e pela captura do nêutron não podiam ser vistos sem o auxílio do líquido cintilante.

Existem vários tipos de cintiladores cujos mecanismos de funcionamento podem variar: cristais inorgânicos, orgânicos, líquidos e polímeros. Para uma descrição mais detalhada nos referimos à Ref. [73].

Além do baixo custo, as vantagens do método são a capacidade de obter a energia do neutrino detectado e o instante da medida. A energia limiar é

\footnotetext{
${ }^{4} \mathrm{~A}$ palavra fluor aqui é utilizada como resumo de fluorescente, para se identificar também com o a terminologia utilizada em textos em inglês. O produto químico é fluorescente, ou seja, emite luz visível quando absorve energia.
} 
pequena, em geral um pouco acima da energia limiar do decaimento $\beta$ inverso $E_{L}=1,8 \mathrm{MeV}$. O método é utilizado principalmente em experimentos com neutrinos de reatores.

\subsubsection{Calorímetros}

Em física de partículas, um calorímetro é um aparato experimental que mede a energia de partículas. Nos experimentos com neutrinos, o calorímetro é normalmente composto de um alvo de grande densidade (alto valor de $\mathrm{Z}$, como o chumbo ou aço) para aumentar a probabilidade de interação com o neutrino.

Quando o neutrino interage cria-se um chuveiro de partículas secundárias cujas energias e trajetórias podem ser medidas. A correlação dos eventos observados indicam a presença indireta e a família do neutrino. Geralmente essas partículas secundárias são observadas com auxílio dos líquidos cintiladores e/ou de TFMs nos experimentos que procuram por neutrinos.

Um dos casos é o experimento MINOS que procura pelos neutrinos produzidos no acelerador do Fermilab [75]. Seus detectores são compostos por camadas alternadas de aço e plástico cintilador. Os pedaços de aços, por terem alta densidade, são colocados para capturar mais facilmente os neutrinos, produzindo assim partículas secundárias. Estas escapam deles e atravessam as camadas ionizando o plástico. Muito parecido também com o experimento de Reines e Cowan, mas no lugar do líquido cintilador é colocado um pedaço de plástico, que atua do mesmo modo. Além disso, a contagem final é feita por TFMs.

Uma outra vantagem deste método, além do alto poder de deteç̧ão do aço, é que ele pode ser magnetizado. Desta forma, o experimento pode identificar nas partículas secundárias múons e antimúons, conseguindo assim distinguir neutrinos de antineutrinos.

Esta tecnologia é particularmente apropriada para a reconstrução de eventos $\nu_{\mu}$ através de CC, porém a identificação de pares elétrons-pósitrons é ruím pois o $e^{-}$não é tão penetrante quanto o $\mu^{-}$. Neste caso, calorímetros com baixos valores de $\mathrm{Z}$ permitiriam boa identificação e medida da energia do $e^{-}$produzidos em interações de $\nu_{e}$ com CC. Nenhum experimento atual se utiliza desta técnica apesar de algumas propostas serem consideradas $[67,76]$. 


\subsection{Resumo}

Ressaltamos aqui as principais características das fontes de neutrinos e seus métodos de observação, facilitando a compreensão e distinção de aplicações.

\subsubsection{Fontes de neutrinos}

\begin{tabular}{|c|c|c|c|}
\hline Fonte & $\begin{array}{l}\text { Reação de } \\
\text { produção }\end{array}$ & $\begin{array}{c}\text { Energia } \\
\text { predominante }\end{array}$ & Observáveis \\
\hline $\begin{array}{c}\text { Atmosférico } \\
\text { (raios cósmicos) }\end{array}$ & $\begin{array}{c}p(\alpha, \ldots)+\mathrm{Ar} \rightarrow \\
\mu^{ \pm}+\nu_{\mu}\left(\bar{\nu}_{\mu}\right)\end{array}$ & $\gtrsim 1 \mathrm{GeV}$ & $R_{\mu / e}$ e $A_{U-D}$ \\
\hline $\begin{array}{c}\text { Solares } \\
\text { (interior do Sol) }\end{array}$ & ciclo $p p$ e $\mathrm{CNO}$ & $\sim(1-10) \mathrm{MeV}$ & fluxo e $A_{N-D}$ \\
\hline Aceleradores & $\begin{array}{c}p(\alpha, \ldots)+\text { Alvo } \rightarrow \\
\mu^{ \pm}+\nu_{\mu}\left(\bar{\nu}_{\mu}\right)\end{array}$ & $\begin{array}{c}\sim \\
(1-100) \mathrm{GeV}\end{array}$ & fluxo \\
\hline Reatores & $n \rightarrow p+e^{-}+\bar{\nu}_{e}$ & $\sim 3 \mathrm{MeV}$ & fluxo \\
\hline Supernova & $\begin{array}{c}\text { várias: } \\
e^{-} p \rightarrow n \nu_{e}, \ldots\end{array}$ & $\sim 15 \mathrm{MeV}$ & $\begin{array}{c}\text { fluxo quando } \\
\text { possível }\end{array}$ \\
\hline Geoneutrinos & $\begin{array}{c}\text { decaimentos do } \\
{ }^{238} \mathrm{U},{ }^{232} \mathrm{Th} \mathrm{e}{ }^{40} \mathrm{~K}\end{array}$ & $\sim 1 \mathrm{MeV}$ & fluxos \\
\hline $\begin{array}{c}\text { Neutrinos cósmicos } \\
\text { de fundo }\end{array}$ & $\begin{array}{l}\text { produzidos no } \\
\text { Big-Bang }\end{array}$ & $\begin{array}{c}\sim \\
1,6 \times 10^{-4} \mathrm{eV}\end{array}$ & Z-bursts \\
\hline
\end{tabular}

Tabela 2.5: Resumo das principais fontes de neutrinos.

\subsubsection{Métodos de detecção}

\begin{tabular}{|c|c|c|c|c|c|c|c|}
\hline Método & Reação & $E_{L}$ & $\mathrm{E}$ & $\mathrm{D}$ & $\mathrm{S}$ & $\mathrm{T}$ & $\mathrm{P} / \mathrm{A}$ \\
\hline \hline Radioquímico & $\nu_{e} X \rightarrow e^{-} Y$ & $\sim 0,1 \mathrm{MeV}$ & - & - & - & - & - \\
\hline $\begin{array}{c}\text { Água } \\
\text { Cherenkov } \\
(+ \text { pesada })\end{array}$ & $\begin{array}{c}\mathrm{ES} \nu+e^{-} \rightarrow \nu+e^{-} \\
\mathrm{NC} \nu+d \rightarrow p+p+e^{-}\end{array}$ & $\sim 5 \mathrm{MeV}$ & $\times$ & $\times$ & $\times$ & $\times$ & - \\
\hline Líq. cintilador & principal: $\bar{\nu}_{e} p \rightarrow e^{+} n$ & $\sim 2 \mathrm{MeV}$ & $\times$ & - & - & $\times$ & - \\
\hline Calorímetro & $\nu_{l}+$ Alvo $\rightarrow l+$ sec. & & $\times$ & $*$ & $\times$ & $\times$ & $*$ \\
\hline
\end{tabular}

Tabela 2.6: Resumo das principais técnicas de observação de neutrinos, com a principal reação da detecção, sua energia limiar e suas características: E, se a energia do neutrino é medida; D, se a direção pode ser inferida; $\mathrm{S}$, se pode distingüir sabores; $\mathrm{T}$, se a medida é feita em tempo real; e $\mathrm{P} / \mathrm{A}$, se o método distingüe partículas de antipartículas. Aqui (-) negação, $(\times)$ afirmação e $\left(^{*}\right)$ alguns casos. A energia limiar apresentada não é exata, pois esta depende não apenas da reação, mas também do experimento, que eleva a mesma afim de reduzir ruídos. 


\section{Capítulo 3}

\section{Experimentos de neutrinos}

Após estudarmos quais as fontes de neutrinos que podemos investigar e os métodos que podemos utilizar na investigação de neutrinos, o presente capítulo apresenta os experimentos realizados que evidenciaram de alguma forma o fenômeno de oscilação (troca de sabor) no setor. No estudo fenomenológico de oscilação de neutrinos, estes são as bases que corroboram ou falseiam os modelos.

Os experimentos de oscilação podem ser divididos em experimentos de desaparecimento ou de aparecimento. Um experimento é dito de desaparecimento quando procura por neutrinos do mesmo sabor daquele produzido na fonte e constata um déficit no fluxo previsto. Ao contrário, um experimento de aparecimento procura por sabores distintos daquele produzido na fonte e encontra um aumento no fluxo previsto.

Esse capítulo divide os experimentos por fonte. Experimentos com características semelhantes são colocados próximos. Por exemplo: o Sol produz $\nu_{e}$ e reatores produzem $\bar{\nu}_{e}$, ambos os experimentos podem medir o mesmo observável já que pelo Teorema CPT, a probabilidade de sobrevivência do neutrino eletrônico é a mesma de seu antineutrino; já os neutrinos atmosféricos e de aceleradores são em grande parte dedicados à medidas da segunda família, $\nu_{\mu}$ ou $\bar{\nu}_{\mu}$.

\subsection{Experimentos de neutrinos solares}

As experiências detectando neutrinos solares foram as primeiras à evidenciar a presença do fenômeno de oscilação no setor. Foram elas que deram início à astrofísica de neutrinos [32].

A rica fenomenologia e metodologia dos poucos experimentos de neutrinos solares (um total de sete) justifica uma exposição mais extensa desses aqui.

O MSP mais recente data de 2005 (BS05 [58]), como comentado no capítulo 
anterior, sendo assim utilizaremos todos os valores previstos para neutrinos solares baseados nesse modelo. Todos os valores aqui apresentados foram obtidos das Refs. [68, 77, 23].

A divisão de apresentação dos experimentos é metodológica e a ordem cronológica. Os resultados mais antigos são colocados na conveniente unidade de neutrino solar (1 SNU $=10^{-36}$ capturas/átomo/s).

Uma fonte de ruído que se encontra em todos os experimentos de neutrinos solares são os múons provenientes de raios cósmicos. Afim de reduzir sua influência nos detectores, estes são instalados à centenas de metros abaixo do solo. A profundidade é $\sim 1000 \mathrm{~m}$ nos experimentos solares e também é medida na sua equivalência em metros d'água $\left(\mathrm{mwe}^{1}\right)$.

\subsubsection{Homestake: experimentos com cloro}

O primeiro resultado de detecção de neutrinos solares foi anunciado por Raymond Davis Jr. e seus colaboradores no laboratório de Brookhaven em 1968 [32].

Localizado na Mina de Ouro de Homestake em Lead, South Dakota, nos Estados Unidos, o experimento tomou dados de 1970 à 1994.

O método empregado era a detecção radioquímica através da reação com cloro: $\nu_{e}+{ }^{37} \mathrm{Cl} \rightarrow^{37} \mathrm{Ar}+e^{-}$. O detector consistia de um tanque com aproximadamente 615 toneladas de $\mathrm{C}_{2} \mathrm{Cl}_{4}$, ou seja, $2,16 \times 10^{30}$ átomos de ${ }^{37} \mathrm{Cl}$ (133 t). A maior fonte de ruídos era a transmutação ${ }^{37} \mathrm{Cl} \rightarrow{ }^{37}$ Ar provocada por neutrinos provenientes de raios cósmicos, para evitar isso o detector foi colocado à $1478 \mathrm{~m}$ de profundidade (4200 mwe), reduzindo o fluxo de múons à apenas $4 \mathrm{~m}^{-2} \mathrm{dia}^{-1}$.

A energia limiar para a reação é $E_{L}^{\mathrm{Cl}}=0,814 \mathrm{MeV}$, então os fluxos solares medidos eram provenientes das reações: pep, hep, ${ }^{7} \mathrm{Be}$ e ${ }^{8} \mathrm{~B}$. De acordo com o MSP, aproximadamente $78 \%$ do número esperado de eventos era devido aos neutrinos do ${ }^{8} \mathrm{~B}$ e $13 \%$ do ${ }^{7} \mathrm{Be}$.

A taxa média de eventos medida e o valor previsto do MSP são, respectivamente,

$$
\begin{aligned}
R_{\mathrm{Cl}} & =2,56 \pm 0,23 \mathrm{SNU} \\
R_{\mathrm{MSP}} & =8,1 \pm 1,3 \mathrm{SNU},
\end{aligned}
$$

ou seja, observou-se um terço da previsão [78].

\footnotetext{
${ }^{1}$ mwe é a abreviatura de equivalentes em metros d'água, cujas iniciais são provenientes da língua inglesa "meters of water equivalent".
} 


\subsubsection{SAGE e GALLEX/GNO: experimentos com gálio}

Os experimentos SAGE [79] e GALLEX [80], além de seu sucessor GNO [81], são baseados no método de detecção radioquímica, com uso do gálio através da reação $\nu_{e}+{ }^{71} \mathrm{Ga} \rightarrow{ }^{71} \mathrm{Ge}+e^{-}$. A energia limiar para que esta ocorra é $E_{L}^{\mathrm{Ga}}=0,233 \mathrm{MeV}$, ou seja, desta forma neutrinos de todas as reações solares podem ser observados.

O resultado final com os três experimentos e a previsão do MSP são, respectivamente, [81]

$$
\begin{aligned}
R_{\mathrm{Ga}} & =72,4 \pm 4,7 \mathrm{SNU} \\
R_{\mathrm{MSP}} & =126 \pm 10 \mathrm{SNU}
\end{aligned}
$$

\section{SAGE: Soviet-American Gallium Experiment}

Localizado em Baksan, Kaberdino-Balkaria, Rússia, tomou dados de 1990 à 2001. O detector era composto de 50 t de gálio metálico líquido. Encontrava-se à 2000 $m$ de profundidade (4700 mwe), com um fluxo de apenas $2,6 \mathrm{~m}^{-2} \mathrm{dia}^{-1}$. As grandes incertezas na seção de choque de detecção foram reduzidas através de calibração direta de neutrinos provenientes do decaimento do ${ }^{51} \mathrm{Cr}$.

De acordo com o MSP, aproximadamente $54 \%$ dos eventos eram devidos aos neutrinos do $p p, 26 \%$ do ${ }^{7} \mathrm{Be}$ e $11 \%$ do ${ }^{8} \mathrm{~B}$.

O resultado obtido foi $R_{\mathrm{SAGE}}=70,8_{-6,1}^{+6,5} \mathrm{SNU}$ [79].

\section{GALLEX: GALLium EXperiment}

Localizado no laboratório de Gran Sasso, Itália, tomou dados de 1991 à 1997. $\mathrm{O}$ detector consistia de $30 \mathrm{t}$ de gálio em 101 t de $\mathrm{GaCl}_{3}-\mathrm{HCl}$. Também foi calibrado com uma fonte de ${ }^{51} \mathrm{Cr}$.

A taxa de neutrinos solares medida foi $R_{\text {GALLEX }}=77,5_{-7,8}^{+7,6} \mathrm{SNU}$ [80].

\section{GNO: Gallium Neutrino Observatory}

Este experimento foi sucessor do GALLEX aumentando a massa de gálio de 30 para 100 t. Dados foram tomados de 1998 à 2000. O resultado obtido foi $R_{\mathrm{GNO}}=65,8_{-10,2}^{+10.7} \mathrm{SNU}[82]$.

A combinação apenas do GALLEX com o GNO fornece $R_{\text {GALLEX/GNO }}=$ $70,8 \pm 5,9$ SNU [82].

\subsubsection{Kamiokande/SK: experimentos com água}

Kamiokande [83] e seu sucessor Super-Kamiokande (SK) [84] localizados na Mina de Kamioka no Japão, à 200 km ao oeste de Tóquio, utilizaram o método de de- 
tecção por radiação Cherenkov. Desta forma, a direção e a energia dos neutrinos também puderam ser investigadas.

A reação envolvida no processo é o espalhamento elástico (ES) $\nu+e^{-} \rightarrow \nu+e^{-}$, sendo assim sensível à todos os sabores de neutrinos. Entretanto as seções de choque para esta reação são diferentes: $\sigma\left(\nu_{e}\right) \simeq 6 \sigma\left(\nu_{\mu, \tau}\right)$. Os detectores se encontravam à $1000 \mathrm{~m}$ abaixo do solo (2700 mwe).

\section{Kamiokande}

O detector de Kamiokande era constituído de 3000 t de água, onde apenas 680 t correspondiam ao volume fiducial ${ }^{2}$. Um total de 948 TFMs foram utilizados. Os dados foram tomados de 1987 à 1995.

A energia limiar do experimento foi ajustada de forma a minimizar os ruídos provenientes de raios cósmicos e outras fontes. Sendo assim, a energia limiar utilizada foi de $E_{L}^{\mathrm{Kam}}=7,5 \mathrm{MeV}$, implicando assim na detecção apenas de neutrinos provenientes do ${ }^{8} B$ e hep. Contudo o fluxo do último não é significativo.

O resultado final do fluxo e seu valor previsto pelo MSP são, respectivamente,

$$
\begin{aligned}
\Phi_{\nu_{e}}^{\mathrm{Kam}} & =2,80 \pm 0,19 \times 10^{6} \mathrm{~cm}^{-2} \mathrm{~s}^{-1} \\
\Phi_{\nu_{e}}^{8 \mathrm{~B}} & =5,69 \pm 0,16 \times 10^{6} \mathrm{~cm}^{-2} \mathrm{~s}^{-1}
\end{aligned}
$$

\section{Super-Kamiokande}

Este experimento sucedeu Kamiokande, aumentando o volume de água e a precisão em uma ordem de grandeza, reduzindo assim a energia limiar.

O volume total passou à ser de 50 kt de água e o fiducial de 22,5 kt, monitorado por 11146 TFMs. A energia limiar utilizada foi na maioria das tomadas de dados de $5 \mathrm{MeV}$.

Os dados foram tomados de 1996 à 2001. O resultado do fluxo de neutrinos eletrônicos é [85]

$$
\Phi_{\nu_{e}}^{\mathrm{SK}}=2,348_{-0,066}^{+0,074} \times 10^{6} \mathrm{~cm}^{-2} \mathrm{~s}^{-1}
$$

Obteve-se também um limite para o fluxo de neutrinos do hep $\Phi_{\text {hep }}^{\mathrm{SK}}<7,9 \Phi_{\text {hep }}^{\mathrm{MSP}}$ (90\% NC).

Além disso, como a direção podia ser medida, a distinção entre neutrinos detectados durante o dia e a noite pôde ser feita. O resultado da assimetria noite-dia é $A_{\mathrm{N}-\mathrm{D}}^{\mathrm{SK}}=0,021 \pm 0,024$. Não houve diferença entre o espectro de energia esperado e medido.

Finalmente, como SK era sensível à direção dos neutrinos, ele pôde mostrar

\footnotetext{
${ }^{2} \mathrm{O}$ volume fiducial é aquele usado na análise de dados.
} 
que seus neutrinos analisados eram necessariamente provenientes do Sol, refutando a idéia da incerteza de sua origem como está na Figura 3.1.

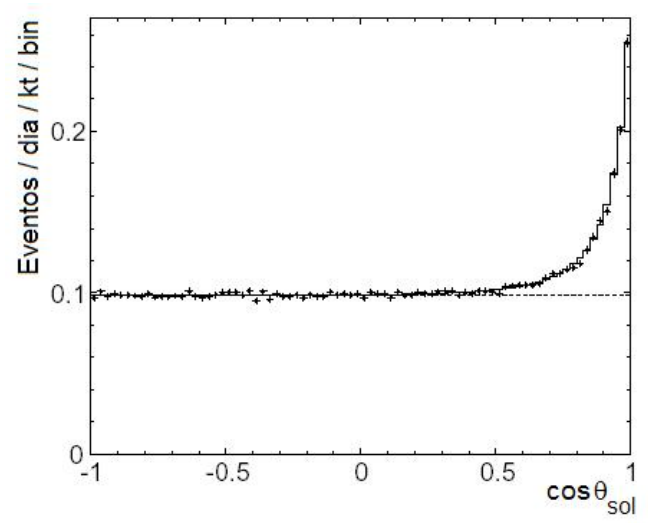

Figura 3.1: Distribuição angular dos eventos do detector SK com respeito à direção do Sol.

\subsubsection{SNO: experimentos com água pesada}

O Sudbury Neutrino Observatory (SNO) foi proposto em 1987 e começou a tomar dados em novembro de 1999 [53]. Ele continua em funcionamento, mas já em junho de 2001 publicou seus primeiros dados na medida de correntes carregadas, e em abril de 2002, os resultados sobre a assimetria noite-dia.

O experimento está localizado na Mina Creighton em Sudbury, Ontario, Canadá. O detector é composto por um 1 kt de água pesada $\left(\mathrm{D}_{2} \mathrm{O}\right)$, e possui instalados 9456 TFMs. Está à 2073 m abaixo do solo (6010 mwe).

Como explicado no Capítulo 2, existem três reações pelas quais o neutrino pode ser capturado por este detector, cujas energias limiares são $E_{L}^{\mathrm{SNO}}(C C) \simeq$ $8,2 \mathrm{MeV}, E_{L}^{\mathrm{SNO}}(N C) \simeq 2,2 \mathrm{MeV}$ e $E_{L}^{\mathrm{SNO}}(E S) \simeq 7,0 \mathrm{MeV}$. Novamente só os neutrinos provenientes do ${ }^{8} \mathrm{~B}$ e hep podem ser detectados. Após a inclusão de sal para redução de incerteza no fluxo NC, os resultados mais recentes são [86]

$$
\begin{gathered}
\Phi_{\mathrm{CC}}^{\mathrm{SNO}}=1,68_{-0,06-0,09}^{+0,06+0,08} \times 10^{6} \mathrm{~cm}^{-2} \mathrm{~s}^{-1} \\
\Phi_{\mathrm{NC}}^{\mathrm{SNO}}=4,94 \pm 0,21_{-0,34}^{+0,38} \times 10^{6} \mathrm{~cm}^{-2} \mathrm{~s}^{-1} \\
\Phi_{\mathrm{ES}}^{\mathrm{SNO}}=2,35 \pm 0,22 \pm 0,15 \times 10^{6} \mathrm{~cm}^{-2} \mathrm{~s}^{-1}
\end{gathered}
$$

A assimetria noite-dia medida é $A_{\mathrm{N}-\mathrm{S}}^{\mathrm{SNO}}=0,037 \pm 0,040$. Não houve discrepâncias entre o espectro de energia previsto e o obtido.

\subsubsection{Futuros experimentos: baixas energias}

O próximo passo que se está dando na investigação de neutrinos solares é a redução da energia limiar de detecção. Com isto, procura-se observar neutrinos 
provenientes de outras reações da cadeia solar, e até mesmo cobrir toda luminosidade de neutrinos.

BOREXino [87] será o primeiro experimento a entrar em operação cujo principal objetivo é a detecção de neutrinos provenientes do berílio. A construção de seu detector deverá terminar neste ano de 2007, no Laboratório de Gran Sasso na Itália. Para alcançar uma energia limiar de $250 \mathrm{keV}$, o experimento se utiliza de 300 t de um líquido cintilador cuja reação de detecção é o espalhamento elástico $\nu e^{-} \rightarrow \nu e^{-}$, sendo assim sensível à todos os sabores.

Uma descrição exaustiva de experimentos de baixas energias como Lithium, CLEAN e outros propostos podem ser encontrada na Ref. [88]. Propõe-se também de aumentar o volume dos detectores à base de água Cherenkov para uma escala de megatoneladas [89, 90, 91].

\subsubsection{Resumo dos resultados}

A Tabela 3.1 apresenta a razão entre o fluxo medido nos diferentes experimentos e o predito pelo MSP (BS05).

Somente a corrente neutra (NC) de SNO, que detecta todos os sabores com mesma seção de choque, apresenta fluxo compatível com o MSP. Os outros resultados apresentam desaparecimento entre $45 \%$ e $70 \%$. Esta variação representa uma dependência energética nos fluxos. Portanto, evidencia-se fortemente a presença de um mecanismo de troca de sabor no setor de neutrino que depende da energia. O mecanismo padrão encontrado no setor de quarks é a oscilação induzida por massa. Da mesma maneira o mesmo pode ocorrer no setor leptônico.

\begin{tabular}{|c|c|c|}
\hline Experimento & Razão (Medido/MSP) & $A_{N-D}$ \\
\hline \hline $\mathrm{Cl}$ & $0,30 \pm 0,03$ & - \\
\hline $\mathrm{Ga}$ & $0,52 \pm 0,03$ & - \\
\hline $\mathrm{SK}$ & $0,413 \pm 0,014$ & $0,021 \pm 0,020 \pm 0,012$ \\
\hline $\mathrm{SNO}(\mathrm{CC})$ & $0,29 \pm 0,02$ & $-0,056 \pm 0,074 \pm 0,053$ \\
\hline $\mathrm{SNO}(\mathrm{NC})$ & $0,87 \pm 0,08$ & $0,042 \pm 0,086 \pm 0,072$ \\
\hline $\mathrm{SNO}(\mathrm{ES})$ & $0,41 \pm 0,05$ & $0,146 \pm 0,198 \pm 0,033$ \\
\hline
\end{tabular}

Tabela 3.1: Resumo dos resultados obtidos para os fluxos nos experimentos de neutrinos solares. O MSP utilizado é BS05 [58].

O resultado combinado de $A_{N-D}^{S K}$ e $A_{N-D}^{S N O}$ fornece $A_{N-D}^{S K+S N O}=0,035 \pm 0,027$ [86].

A Figura 3.2 apresenta os principais resultados dos experimentos de neutrinos solares juntamente com a previsão destes com a hipótese de oscilação, cuja análise será discutida no próximo capítulo. 


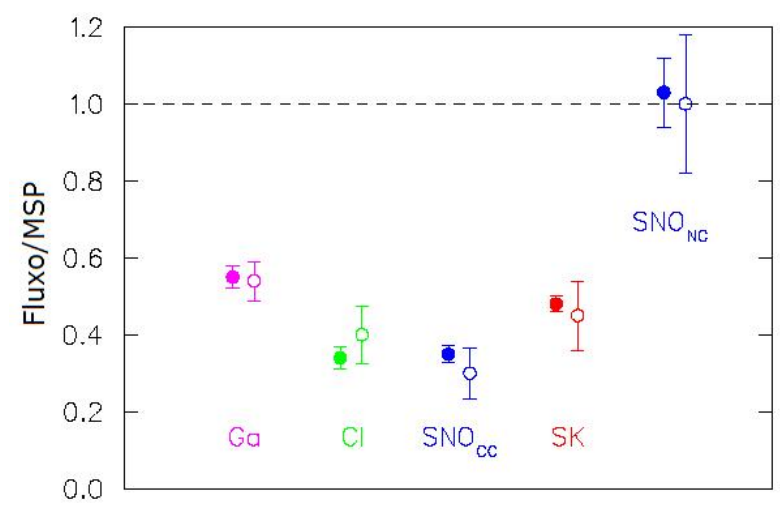

Figura 3.2: Resultados obtidos nos experimentos de neutrinos solares. Os círculos cheios são os valores experimentais, e os vazios as previsões com oscilação.

\subsection{Experimentos de neutrinos em reatores}

Os experimentos que procuram por neutrinos de usinas nucleares são divididos naqueles de curtas distâncias $\left(\mathrm{SBL}^{3}\right)$, e de longas distâncias $\left(\mathrm{LBL}^{4}\right)$, entre os reatores e os detectores.

Todos os experimentos realizados em reatores se utilizam da técnica do líquido cintilador como alvo, dopado de um elemento para acelerar a captura do nêutron: ${ }^{3} \mathrm{He},{ }^{6} \mathrm{Li}$ ou Gd. A vantagem do gadolínio é sua grande seção de choque de captura de nêutrons e a emissão de raios $\gamma$ com altas energias $\sim 8 \mathrm{MeV}$, bem acima da radioatividade natural. Uma única excessão é o experimento mais recente, KamLAND, cuja densidade de prótons do alvo é tão grande que não necessita de dopagem.

Uma grande consideração deve ser dada aos ruídos, que podem ser classificados por duas fontes distintas [92]:

1. ruídos internos devidos à radioatividade dos materias do detector (por exemplo a atividade do ${ }^{40} \mathrm{~K}$ dos TFMs) e do local do laboratório;

2. atividade externa, como os raios cósmicos. Neste caso, novamente os detectores são instalados em minas com grande profundidade, e além disso dentro de alguma proteção com materiais de baixa atividade radioativa como areia ou molde de ferro, ou que sirvam de bloqueio, como água ou outro material detector.

As proteções tem uma eficiência acima de $90 \%$ em todos os projetos realizados.

A energia média dos neutrinos observados está entre 3 e $5 \mathrm{MeV}$, dentro de um espectro que costuma variar entre 2,5 e $10 \mathrm{MeV}$, já que a energia limiar do decaimento $\beta$ inverso é 1,8 MeV e geralmente os detectores não detectam o pósitron

\footnotetext{
${ }^{3}$ do inglês "Short BaseLines", ou seja, curta distância percorrida.

${ }^{4}$ do inglês "Long BaseLines", ou seja, longa distância percorrida.
} 
com energia inferior à $0,7 \mathrm{MeV}$. À essas baixas energias, os únicos léptons que podem ser produzidos em interações CC nos detectores são os elétrons, portanto os experimentos de neutrinos de reatores são de desaparecimento.

\subsubsection{ILL, Bugey, Gosgen, Krasnoyarsk, Rovno, Savannah River: detectores de SBL}

Durante as décadas de 80 e 90, motivados pelos resultados dos neutrinos solares, realizaram-se experimentos com neutrinos de reatores buscando encontrar mais evidências de oscilação. Na tentativa de maximizar a estatística dos dados, os primeiros projetos colocaram seus detectores próximos aos núcleos dos reatores. A distância utilizada costumava estar dentro de $100 \mathrm{~m}$. As vezes, no mesmo experimento, várias distâncias eram usadas afim de reduzir o erro sistemático, que dominava sobre os estatísticos (veja Tabela 3.2).

Normalmente, apenas uma usina com um reator de baixa potência térmica (entre $57 \mathrm{MW}$ e $3 \mathrm{GW}$ ) era investigada. Ainda nesses experimentos de SBL, pouca proteção aos ruídos é necessária, a profundidade típica é $\sim 10$ mwe. O volume dos detectores era $\sim 0,5 \mathrm{t}$.

Todos os resultados obtidos são compatíveis com os valores previstos no MP sem desaparecimento, e com um fluxo proporcional ao inverso do quadrado da distância.

Apenas uma curiosidade histórica vale lembrar aqui, o experimento Bugey divulgado em 1984 [93]. Foi o único do gênero que relatou um indício de desaparecimento, e isto fez com que mais grupos de pesquisa trabalhasse no assunto. Os resultados atuais do projeto se baseiam na coleta de 1993 [70], onde 3 distâncias foram utilizadas, o chamado Bugey 3, que não confirmou os dados de 1984.

\subsubsection{CHOOZ, KamLAND, Palo Verde: detectores de LBL}

Os resultados encontrados nos experimentos de SBL foram interpretados como um conhecimento teórico preciso dos reatores como fonte de neutrinos. Isso ajudou, e obrigou, os projetos a colocarem os detectores à distâncias maiores com intuito de se encontrar indícios de desaparecimento de neutirnos.

Apenas três experimentos de LBL foram realizados até hoje: CHOOZ, Palo Verde e KamLAND. 


\section{CHOOZ e Palo Verde}

Os experimentos de CHOOZ [94] e Palo Verde [95] apresentam características semelhantes, além do mesmo método de detecção com líquido cintilador dopado com quase $1 \%$ de gálio.

A potência térmica dos dois reatores em CHOOZ e três em Palo Verde representam respectivamente 8,5 GW e 11,63 GW. Ambas as distâncias são de aproximadamente $1 \mathrm{~km}$. Apesar de ter um volume maior em Palo Verde, com 11,3 t, enquanto CHOOZ tem 5 t, uma maior proteção aos ruídos foi construída para esse último fazendo com que a taxa efetiva de captura de neutrinos fosse quase cinco vezes maior, de 51 para 12 neutrinos/dia/t/GW.

Os resultados estão na Tabela 3.2 sendo também compatíveis com o MP.

\section{KamLAND}

KamLAND é um experimento único de grandes proporções [96]. Localizado na mina de Kamioka, conta com neutrinos provenientes de 16 usinas nucleares no Japão e 4 na Coréia do Sul, somando um total de 69 reatores. Este valor representa uma potência térmica $\sim 80 \mathrm{GW}$ que varia dependendo das condições de operações dos reatores.

A distância do detector aos núcleos dos reatores varia de 80 à 344 km. A distância efetiva calculada, cuja média é $\sim 180 \mathrm{~km}$, também varia com as condições de operação. Assim, dois resultados obtidos com dados em diferentes épocas do ano podem representar distâncias efetivas diferentes, e não devem ser comparados diretamente.

O detector se encontra à 2700 mwe, mas pode detectar geoneutrinos com energia $E_{g} \leq 2,49 \mathrm{MeV}$. Assim um corte de $E_{L}=2,6 \mathrm{MeV}$ é colocado. Um maior conhecimento do detector levou o grupo à utilizar um volume fiducial de 543,7 t na sua última análise de dados. Considerando mais fontes de ruídos que anteriormente, os dois resultados divulgados se baseiam na tomada de dados em duas época distintas [97, 98]. Eles estão apresentados na Tabela 3.2.

KamLAND é atualmente o único experimento de reator que confirma com nível de significância maior que $99 \%$ o desaparecimento de $\bar{\nu}_{e}$.

\subsubsection{Futuros experimentos: detectores distantes}

O futuro da física de neutrinos de reator está na construção de experimentos de LBL, com um detector próximo para normalização e outro bem distante. Esta é a melhor maneira de se encontrar evidências de oscilação o que possibilitará medir o ângulo de mistura $\theta_{13}$ que como veremos no capítulo seguinte só possui 
um limite superior [99].

Três detectores já estão sendo contruídos: Daya Bay [100], na China; DoubleCHOOZ [101], na França; e Reno, na Coréia do Sul.

Essa investigação pode revelar mais informações sobre a física além do MP, como a violação da simetria CP no setor leptônico, e por estes motivos vários outros projetos já foram propostos: Angra, Braidwood, Diablo Canyon, KASKA, Kr2Det entre outros lugares, numa carta branca assinada por diversos físicos da área (veja Ref. [92, 99, 102]).

\subsubsection{Resumo dos resultados}

A Tabela 3.2 resume os resultados obtidos em todos os experimentos de neutrinos de reatores.

\begin{tabular}{|c|c|c|c|}
\hline Nome & Localização & Data & Razão (Medido/Previsto) \\
\hline ILL [103] & $\begin{array}{l}\text { Grenoble, } \\
\text { França }\end{array}$ & 1981 & $R_{9 \mathrm{~m}}=0,955 \pm 0,035 \pm 0,11$ \\
\hline Gösgen [104] & $\begin{array}{l}\text { Gösgen, } \\
\text { Suiça }\end{array}$ & 1986 & $\begin{array}{l}R_{38 \mathrm{~m}}=1,018 \pm 0,019 \pm 0,075 \\
R_{46 \mathrm{~m}}=1,045 \pm 0,019 \pm 0,075 \\
R_{65 \mathrm{~m}}=0,975 \pm 0,036 \pm 0,090\end{array}$ \\
\hline Rovno [105] & $\begin{array}{l}\text { Rovno, } \\
\text { Ucrânia }\end{array}$ & 1987 & $R_{18 \mathrm{~m}}=0,995 \pm 0,060$ \\
\hline Krasnoyarsk [106] & Rússia & 1994 & $R_{57 \mathrm{~m}}=0,99 \pm 0,05$ \\
\hline Bugey 3 [70] & $\begin{array}{l}\text { Bugey, } \\
\text { França }\end{array}$ & 1995 & $\begin{array}{l}R_{15 \mathrm{~m}}=0,988 \pm 0,004 \pm 0,05 \\
R_{40 \mathrm{~m}}=0,994 \pm 0,010 \pm 0,05 \\
R_{95 \mathrm{~m}}=0,915 \pm 0,132 \pm 0,05\end{array}$ \\
\hline Savannah R. [107] & $\begin{array}{l}\text { Aiken, } \\
\text { EUA }\end{array}$ & 1996 & $\begin{array}{l}R_{18 \mathrm{~m}}=0,987 \pm 0,006 \pm 0,037 \\
R_{24 \mathrm{~m}}=1,055 \pm 0,001 \pm 0,037\end{array}$ \\
\hline CHOOZ [108] & $\begin{array}{l}\text { Chooz, } \\
\text { França }\end{array}$ & 2003 & $R_{1 \mathrm{~km}}=1,01 \pm 0,028 \pm 0,027$ \\
\hline Palo Verde [109] & $\begin{array}{l}\text { Palo Verde, } \\
\text { EUA }\end{array}$ & 2001 & $R_{1 \mathrm{~km}}=1,01 \pm 0,024 \pm 0,053$ \\
\hline KamLAND $[97,98]$ & $\begin{array}{l}\text { Kamioka, } \\
\text { Japão }\end{array}$ & 2004 & $\begin{array}{l}R_{2002}=0,589 \pm 0,085 \pm 0,042 \\
R_{2004}=0,658 \pm 0,044 \pm 0,047\end{array}$ \\
\hline
\end{tabular}

Tabela 3.2: Resumo dos experimentos de neutrinos de reatores com as razões entre fluxos obtidos e esperados obtidas, além das datas de publicação dos dados.

O único experimento que apresenta desaparecimento de antineutrinos eletrônicos é KamLAND, com déficit de quase $40 \%$. 
A Figura 3.3 apresenta os principais resultados dos neutrinos de reatores e sua previsão com oscilação.

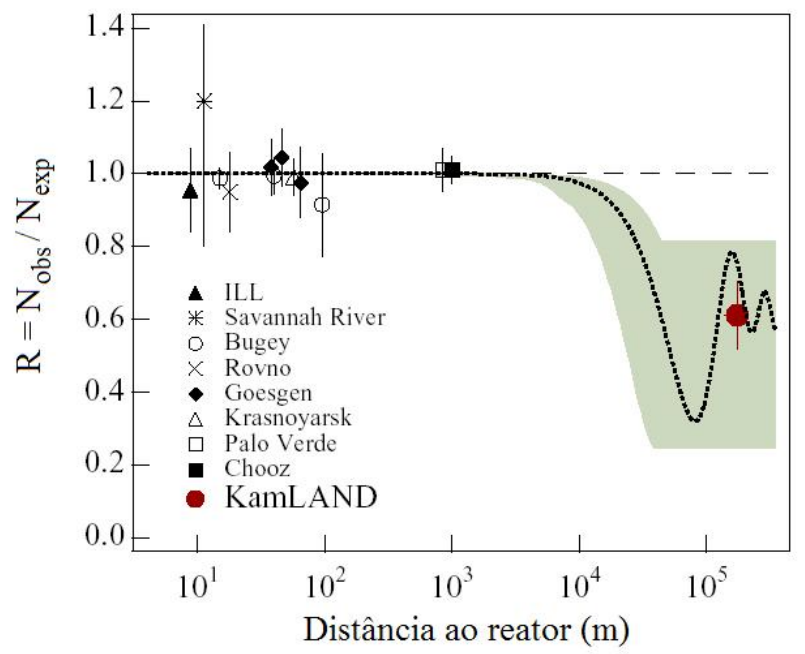

Figura 3.3: Resultados dos experimentos de neutrinos de reatores. A linha pontilhada corresponde ao valor previsto com oscilação, e a hachurada sua região com $95 \%$ de confiança.

\subsection{Experimentos de neutrinos atmosféricos}

Os experimentos com neutrinos atmosféricos começaram na década de 1960 [110]. O objetivo principal destes experimentos era apenas a confirmação da existência de neutrinos nos raios cósmicos, mas observaram evidências de oscilação.

No começo dos anos 80, experimentos maiores (mais massivos) localizados no subsolo foram construídos na procura do decaimento do próton, cuja meia vida de $\lesssim 10^{32}$ anos era prevista pelas primeiras Teorias de Grande Unificação. Entretanto o ruído mais sério era os eventos de neutrinos atmosféricos, com uma taxa de $10^{2}$ eventos/ano/kt. Isto os conduziu à um estudo mais detalhado destes neutrinos observados. Hoje, além destes primeiros experimentos usando calorímetros, foram construídos grandes detectores com água que utilizam o método da radiação Cherenkov para detecção.

Em todos os experimentos, os eventos podem ser classificados em três categorias:

1. $\mathrm{FC}^{5}$ : em que todas as partículas finais se encontram dentro do detector;

2. $\mathrm{PC}^{6}$ : quando $\nu_{\mu}$ possue mais energia e o múon escapa do detector;

\footnotetext{
${ }^{5}$ do inglês "fully contained".

${ }^{6}$ do inglês "partially contained".
} 
3. $\mathrm{UG}^{7}$ : se as interações ocorrem na vizinhança do detector, e o múon entra e atravessa (TH-UG) ou pára no detector (ST-UG). Estes últimos múons são entendidos provenientes de baixo pois deve haver suficiente quantidade de matéria para absorvê-los dos raios cósmicos.

A dependência energética desta classificação de eventos pode ser vista na Figura 3.4 .

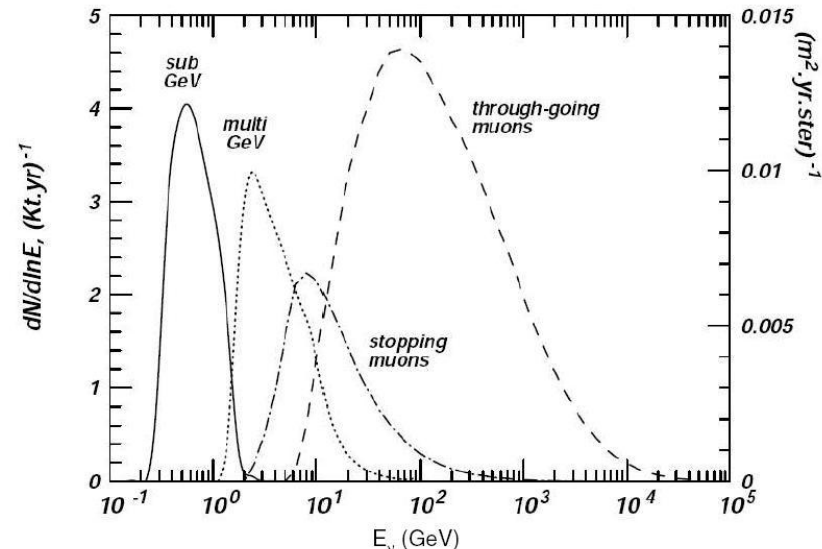

(a)

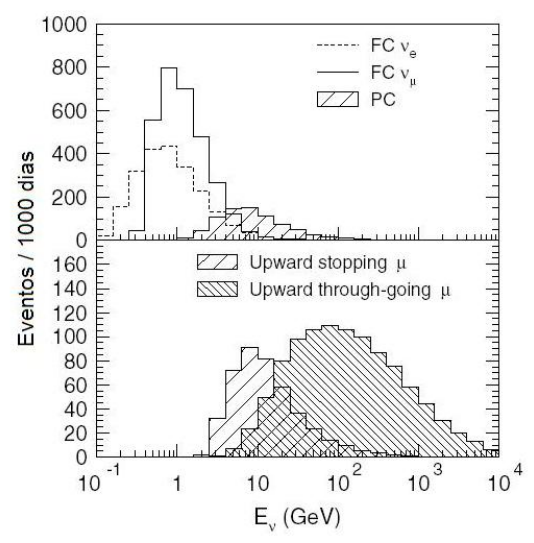

(b)

Figura 3.4: A distribuição de energia para as diferentes classificações dos eventos. Estes espectros são provenientes do experimento de SK; para outros detectores eles serão parecidos, mas dependem do tamanho do detector [111]. A classificação em (a) com eventos sub-GeV, multi-GeV e UG, e em (b) com eventos FC, PC e UG.

Os múons provenientes dos raios cósmicos são aqui também a maior fonte de ruído nos experimentos, pois os hádrons, elétrons e raios gamas são rapidamente absorvidos por alguns metros de rocha. Então para diminuir significativamente o fluxo de múons, o detector deve estar localizado bem abaixo do solo. A Figura 3.5 mostra a profundidade de alguns detectores e a taxa de múons dos raios cósmicos para essas profundidades.

Além disso, outra proteção colocada em alguns experimentos são os chamados vetos ou antidetectores, que podem facilmente tanto identificar as partículas saindo quanto rejeitar algum ruído entrando. Em geral são consituídos de outro tipo de detector colocado em volta do detector principal.

Pela dificuldade e incerteza na realização de alguns cálculos, o principal parâmetro medido nestes experimentos não é o fluxo, e sim a taxa entre neutrinos muônicos e eletrônicos:

$$
R=\frac{\left(\frac{\nu_{\mu}+\bar{\nu}_{\mu}}{\nu_{e}+\bar{\nu}_{e}}\right)_{\exp }}{\left(\frac{\nu_{\mu}+\bar{\nu}_{\mu}}{\nu_{e}+\bar{\nu}_{e}}\right)_{\text {teo }}}
$$

\footnotetext{
${ }^{7}$ do inglês "upward-going muon".
} 


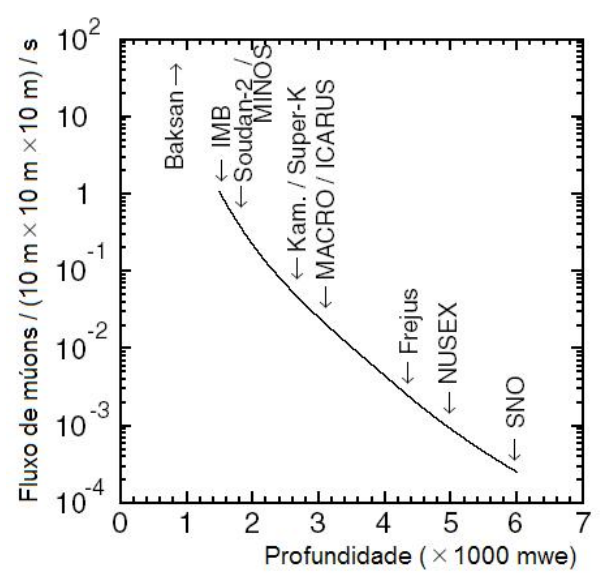

Figura 3.5: Profundidade dos detectores e as taxas calculadas de múons de raios cósmicos [111].

cuja previsão de MC está representada na Figura 3.6.

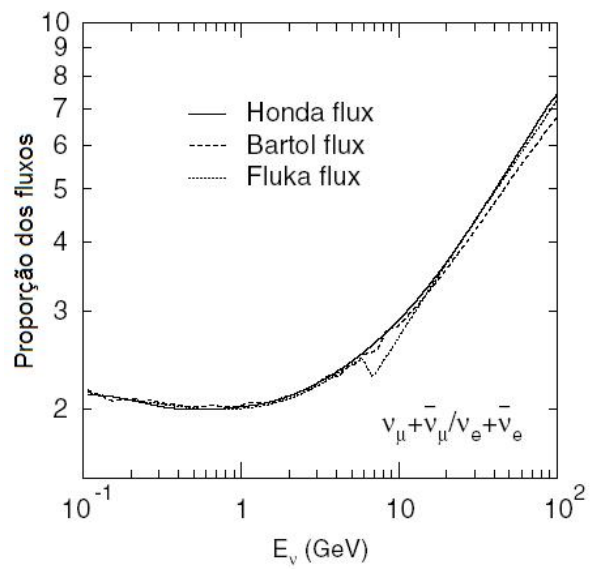

Figura 3.6: A proporção entre os fluxos $\nu_{\mu}+\bar{\nu}_{\mu}$ e $\nu_{e}+\bar{\nu}_{e}$ em função da energia dos neutrinos. Linhas sólidas, tracejadas e pontilhadas são as previsões feitas por [112], [113] e [114] respectivamente.

Não é feita a distinção entre neutrinos e antineutrinos, com excessão de alguns resultados bem recentes e com pouca estatística ainda $[115,116]$. Outro parâmetro que alguns experimentos que possuem maior resolução espacial podem analisar é o da simetria $A_{U-D}$ discutida no capítulo anterior.

Até a presente data apenas dois métodos foram empregados na procura pelos neutrinos atmosféricos: calorimetria e radiação Cherenkov. Como explicado no Capítulo 2, ambos os métodos são capazes de medir sabor, energia e direção. As principais características dos experimentos e seus resultados são apresentadas na Tabela 3.3. 


\subsubsection{Experimentos com calorímetros: KGF, Fréjus, Bak- san, Soudan 2, NUSEX, MACRO e MINOS}

Experimentos com calorímetros foram os primeiros empregados na detecção de neutrinos atmosféricos. A utilização de calorímetros tem a vantagem de detectar neutrinos com energia abaixo do limiar para a radiação Cherenkov. Novos experimentos foram executados e hoje eles podem ser divididos em três gerações.

\section{Primeira geração: Soudan 2}

A primeira geração inclui KGF [117], NUSEX [118], Fréjus [119] e Soudan 1 e 2 [120]. Estes detectores eram menores que os atuais com massa $\sim 1 \mathrm{kt}$. Os primeiros resultados foram obtidos por Fréjus e NUSEX, e são compatíveis com a previsão de MC. Sua inconsistência com os resultados obtidos nos detectores Cherenkov à água levou à uma dicotomia, que hoje é interpretada como devida à baixa estatística dos eventos medidos por esses experimentos uma vez que os resultados de Soudan 2, o principal experimento desta geração, e MACRO, da geração seguinte concordam com os experimentos em água.

A alta precisão obtida pelo calorímetro de aço instalado em Soudan foi conseguida por três particularidades. O experimento era capaz de reconstruir a trajetória de recúo dos prótons nas reações $\nu_{\mu} n \rightarrow \mu^{-} p$. Ele também era equipado com um anticontador útil na estimativa dos ruídos. Além disso, o método de análise foi escolhido para emular as análises usadas pelos detectores de água Cherenkov, onde somente eventos com trajetórias simples (múons) e chuveiros simples (elétrons) são contados.

Como mencionado, os resultados de Soudan 2 não confirmou aqueles de Fréjus e NUSEX.

\section{Segunda geração: MACRO}

A segunda geração conta com detectores maiores $\sim 5 \mathrm{kt}$ e é mais sensível aos neutrinos do múon da forma UG. É representada pelos experimentos de Baksan [121] e, principalmente, MACRO [122].

MACRO foi um detector de subsolo desenvolvido com vários propósitos na busca de fenômenos raros na radiação cósmica. Um deles era a procura de monopólos magnéticos, e por isso possui alta resolução espacial de até $0,3 \mathrm{~cm}$.

MACRO detectou UG com stream tube system (para reconstrução de trajetórias) e um sistema cintilante (para medida de tempo de vôo). 


\section{Terceira geração: MINOS}

Existe ainda uma última geração de detectores de neutrinos atmosféricos que inclui uma reconstrução de trajetória magnética. Por enquanto existe apenas MINOS nesta geração.

MINOS foi projetado, sobretudo, para operar com um feixe de acelerador. Seu detector foi descrito nos Métodos de Observação do Capítulo 2. Uma de suas particularidades é a capacidade de distinção entre neutrinos do múon e seus antineutrinos. No final de 2005, o grupo publicou uma primeira análise com estes eventos. A proporção de eventos $\bar{\nu}_{\mu}$ para $\nu_{\mu}$ nos dados é comparada com a previsão de MC assumindo que neutrinos e antineutrinos oscilam da mesma maneira. O resultado obtido foi $R_{\bar{\nu}_{\mu} / \nu_{\mu}}^{\text {dados }} / R_{\bar{\nu}_{\mu} / \nu_{\mu}}^{\mathrm{MC}}=0,96_{-0,27}^{+0,38} \pm 0,15$, que apesar da baixa estatística, é a primeira observação direta de interações de neutrinos separadamente para $\nu_{\mu}$ e $\bar{\nu}_{\mu}$ [115]. Em janeiro de 2007 este resultado foi atualizado para $R_{\bar{\nu}_{\mu} / \nu_{\mu}}^{\text {dados }} / R_{\bar{\nu}_{\mu} / \nu_{\mu}}^{\mathrm{MC}}=0,72_{-0,12-0,04}^{+0,24+0,08}$ [116] que é consistente com a conservação da simetria CPT.

\subsubsection{Experimentos com água: Kamiokande/SK e IMB}

Apenas três experimentos de neutrinos atmosféricos utilizam radiação Cherenkov na água. Estes também podem ser divididos em duas gerações de acordo com seu tamanho. Na primeira geração encontram-se os detectores de IMB e Kamiokande. Com um porte maior, apenas SK se inclue na segunda geração.

\section{IMB e Kamiokande}

IMB, sigla de Irvine-Michigan-Brookhaven, tomou dados entre 1982 e 1991 após duas grandes atualizações [123]. O IMB 3 contava com 8 kt de massa total cuja parte fiducial representava $3,3 \mathrm{kt}$. Um total de $2048 \mathrm{TFMs}$ foram utilizados neste experimento.

Kamiokande, descrito em 3.1.3, era um pouco menor. Entretanto, contava com um detector externo que tornava possível identificar eventos PC [124, 125].

Ambos experimentos mostraram que a fração observada de múons em relação à elétrons era muito menor que a previsão por Monte Carlo. Os resultados de Kamiokande para eventos multi-GeV (acima de 1,33 GeV) e PC sugeriram uma dependência no ângulo zenital que indicava a presença de oscilação.

\section{Super-Kamiokande}

A atual geração é o SK [126, 127] que domina nossa compreensão de neutrinos atmosféricos. Em junho de 1998, na conferência Neutrino98, SK apresentou 
evidências de oscilação de $\nu_{\mu}$ baseadas na distribuição angular de sua amostra de eventos FC.
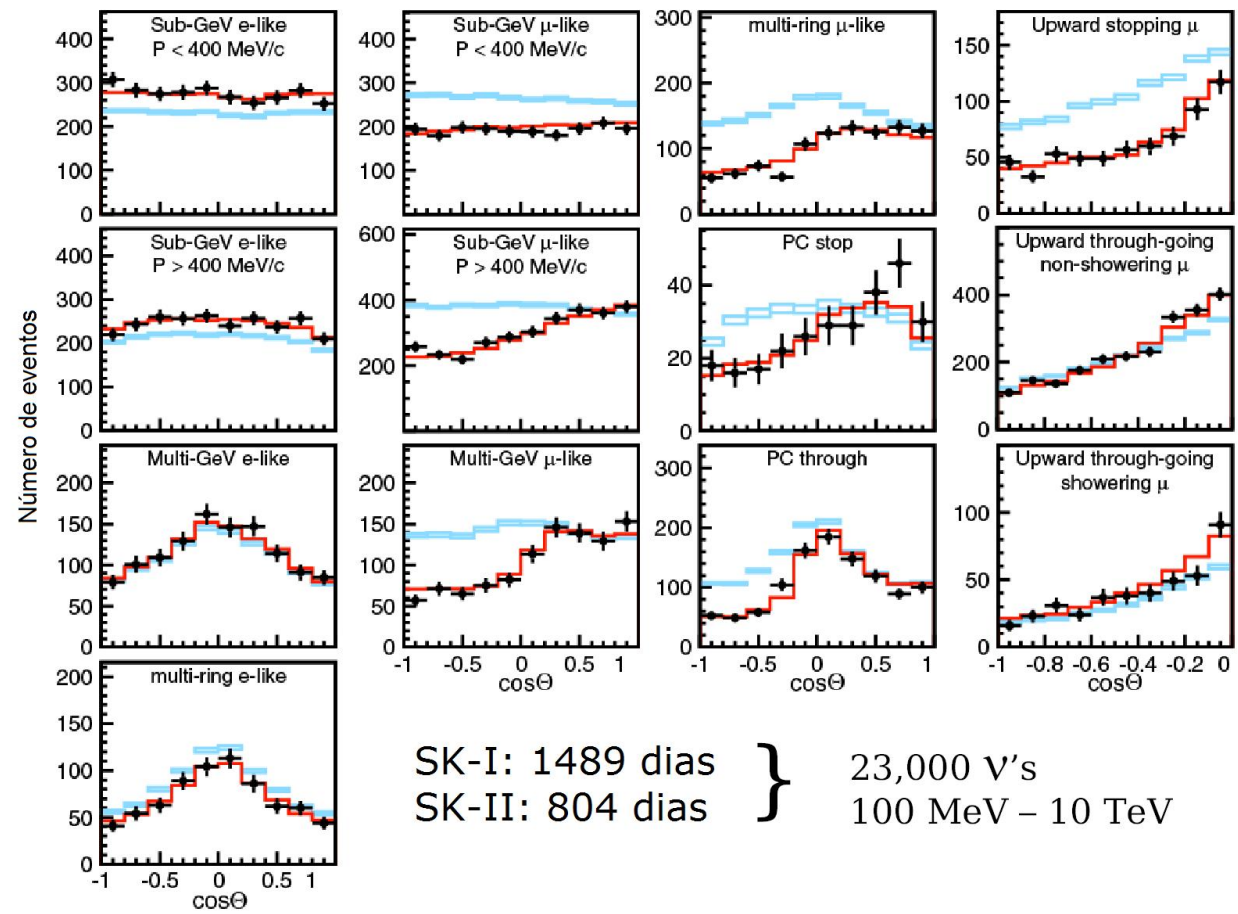

Figura 3.7: Distribuição angular obtida no experimento de SK para os eventos FC, PC e UG. Os pontos representam os dados experimentais, os blocos a previsão de MC sem oscilação e as linhas o melhor valor ajustado com oscilação.

Desde então, SK acumulou muito mais estatística e seus resultados mais importantes são as taxas de fluxos com dependência angular. A Figura 3.7 apresenta os resultados atualizados [128]. A partir dos gráficos, as seguintes observações podem ser feitas:

- o déficit de neutrinos atmosféricos deve-se principalmente ao desaparecimento de $\nu_{\mu}$ e não ao aparecimento de $\nu_{e}$, já que as distribuições do último são bem descritas por MC;

- a assimetria obtida foi $A_{U-D}^{S K}=-0,29 \pm 0,03$, diferindo do valor nulo previsto por 10 desvios padrões. A dependência angular implica que o déficit de neutrinos cresce com a distância percorrida, ou seja, para grandes valores de $\cos \theta$;

- o déficit de múons TH-UG é menor, o que implica que a grandes energias o neutrino tem menos chance de desaparecer. 


\subsubsection{Futuros experimentos: grandes calorímetros e detec- tores Cherenkov de megatoneladas}

Os experimentos futuros de detecção de neutrinos atmosféricos incluem tanto projetos de calorímetros quanto de Cherenkov em água.

Existem planos para se construir um calorímetro de aço magnetizado de 3050 kt na Índia dentro do projeto India-based Neutrino Observatory (INO) [129] cujo objetivo principal é o estudo de oscilação de neutrinos atmosféricos $\nu_{\mu} \mathrm{e}$ $\bar{\nu}_{\mu}$. Embora não existam estudos detalhados das capacidades de tal detector, sua sensibilidade deverá ser comparável àquela obtida numa proposta anterior para um detector parecido denominado MONOLITH [130].

Como comentado anteriormente, uma próxima geração com detectores à base de água Cherenkov está sendo proposta nos EUA, no Japão e na Europa [89, 91]. Apesar desses detectores terem vários propósitos como a procura do decaimento do próton, eles deverão investigar com sensibilidade maior a de SK os parâmetros de oscilação para neutrinos atmosféricos, ou mesmo solar e de acelerador. Além disso, eles poderão trazer mais informações sobre neutrinos de explosões de supernova e neutrinos relíquias de supernova.

\subsubsection{Resumo dos resultados}

A Tabela 3.3 resume os resultados obtidos nos experimentos de neutrinos atmosféricos para a taxa $R=\frac{\left(\frac{\nu_{\mu}+\bar{\nu}_{\mu}}{\nu_{e}+\bar{\nu}_{e}}\right)_{\exp }}{\left(\frac{\nu_{\mu}+\bar{\nu}_{\mu}}{\nu_{e}+\bar{\nu}_{e}}\right)_{\text {teo }}}$.

\begin{tabular}{|c|c|c|c|c|}
\hline Experimento & Data & Localização & Tipo de evento & R (Medido/MC) \\
\hline \hline Fréjus & $1984-1988$ & França/Itália & FC e PC & $1,00 \pm 0,15 \pm 0,08$ \\
\hline NUSEX & $1982-1991$ & Itália & FC & $0,96_{-0,28}^{+0,32}$ \\
\hline Soudan 2 & $1989-2001$ & EUA & FC e PC & $0,64 \pm 0,11 \pm 0,06$ \\
\hline Baksan & $1978-$ & Rússia & TH-UG & não calculado \\
\hline MACRO & $1988-2001$ & Itália & TH-UG & $0,72 \pm 0,026 \pm 0,043$ \\
\hline MINOS & $2002-$ & EUA & - & sem resultado \\
\hline IMB 3 [123] & $1982-1991$ & EUA & sub-GeV & $0,71 \pm 0,04 \pm 0,08$ \\
\hline & & & multi-GeV & $1,1_{-0,12}^{+0,07} \pm 0,11$ \\
\hline Kamiokande 3 & $1983-1986$ & Japão & sub-GeV $[124]$ & $0,60_{-0,06}^{+0,07} \pm 0,05$ \\
\hline & & & multi-GeV $[125]$ & $0,57_{-0,07}^{+0,08} \pm 0,02$ \\
\hline SK & $1996-$ & Japão & sub-GeV $[126]$ & $0,61 \pm 0,03 \pm 0,05$ \\
\hline & & & multi-GeV $[127]$ & $0,66 \pm 0,06 \pm 0,08$ \\
\hline
\end{tabular}

Tabela 3.3: Resumo dos resultados obtidos em experimentos de neutrinos atmosféricos. 
O resultado mais preciso para a simetria U-D também é devido à SK: $A_{U-D}^{S K}=$ $-0,29 \pm 0,03$.

\subsection{Experimentos de neutrinos em aceleradores}

Os neutrinos produzidos em aceleradores possuem altas energias, como foi visto na seção de neutrinos de aceleradores do Capítulo 2. Além disso, por serem produzidos em laboratórios, diversos tipos de experimentos podem ser executados. Isso tudo tem como consequência que um número realmente elevado de experimentos que procuram por neutrinos de aceleradores já foram realizados. Desta forma, não cabe na presente seção fazer uma lista exaustiva dos mesmos como feito nos casos de neutrinos solares, atmosféricos e de reatores.

Os experimentros de neutrinos em aceleradores também podem ser classificados de SBL e LBL. No caso, experimentos de SBL costumam apresentar uma distância entre o acelerador e o detector $\sim 10^{2}-10^{3} \mathrm{~m}$, enquanto os de LBL apresentam $\sim 10^{2} \mathrm{~km}$. Além dessa classificação, por sua vasta quantidade, existem tanto os experimentos de aceleradores de desaparecimento quanto os de aparecimento.

Uma das principais diferenças entre detectores de neutrinos em aceleradores e outros detectores de alvo fixo ou colisões é que o ponto de interação do neutrino não é bem conhecido. Portanto, o detector como um todo funciona como alvo. E como já foi explicado, um grande e denso detector é necessário para obter um número razoável de eventos.

Um detector ideal de neutrinos de aceleradores deveria satisfazer os seguintes requisitos [131]:

- um alvo de massa grande (da ordem de toneladas ou kilotoneladas) para obter uma taxa significativa de eventos;

- boa identificação dos léptons carregados (principalmente múon e elétron) para distinguir $\nu_{\mu}$ e $\nu_{e}$;

- identificação das cargas dos léptons para separar neutrinos de antineutrinos;

- medida completa do quadrivetor energia-momento para os léptons e sistemas hadrônicos de forma à determinar todas as variáveis cinemáticas;

- identificação dos hádrons individuais e de partículas de curta meia-vida para medir os estados finais exclusivos, tais como $K^{0}, \tau$, ou produção do charm. 
Na prática, é muito difícil alcançar todos esses requisitos. Como os múons são partículas penetrantes, essencialmente todos os detectores de neutrinos de aceleradores conseguem identificar múons de altas energias.

As maiores taxas de eventos são obtidas com calorímetros de rastreamento de aço, sacrificando a identificação dos elétrons e dos hádrons individuais por um detector com massa grande. Exemplos deste tipo de detector são: CDHS [132] no CERN, o CCFR/NuTeV [133] no Fermilab e o MINOS [134] em Soudan nos EUA.

Na busca de maior resolução na trajetória individual costuma-se empregar as câmaras de bolhas, como o BEBC [135] no CERN, e emulsão nuclear, cujos exemplos incluem E531 [136] no Fermilab, CHORUS [137] no CERN, e OPERA [138] em Gran Sasso.

Finalmente, alguns detectores são otimizados na detecção do elétron usando alvos relativamente mais leves. Os detectores CHARM/CHARM II [139] e NOMAD [140] no CERN são exemplos desse tipo.

\subsubsection{Experimentos de SBL}

Os primeiros experimentos de neutrinos em aceleradores, e a maior parte deles, foram realizados com SBL. As distâncias características nesse caso são da ordem de algumas centenas de metros.

Com excessão do experimento de LSND, e relacionados, que serão discutido mais abaixo, todas as procuras por troca de sabor tiveram resultados negativos. A Tabela 3.4 apresenta os principais experimentos e resultados. Uma lista completa dos experimentos nesse contexto é muito extensa e pode ser encontrada na Ref. [141].

\section{LSND, KARMEN e MiniBooNE}

LSND (Liquid Scintillator Neutrino Detector) no acelerador linear Los Alamos Meson Physics Facility LAMPF/LANSCE, EUA, e KARMEN (KArlsruhe Rutherford Medium Energy Neutrino) no ISIS em Rutherford Appleton Laboratory, UK, são experimentos otimizados para a detecção de neutrinos na faixa 10-100 MeV. Em ambos os aceleradores, os neutrinos são produzidos pela colisão de prótons de $800 \mathrm{MeV}$ com um alvo.

O detector LSND [147] era constituído por um tanque de $\approx 210 \mathrm{~m}^{3}$ preenchido com baixa concentração de um cintilador líquido dissolvido em óleo mineral que permitia a detecção via luz Cherenkov e cintilação. O detector ficava localizado $30 \mathrm{~m}$ abaixo da fonte de neutrinos. O experimento foi o único a observar um excesso de eventos comparados com o ruído esperado, consistente com a troca de 


\begin{tabular}{|c|c|c|c|c|}
\hline Experimento & Feixe & Canal & Energia $(\mathrm{GeV})$ & Resultado $(90 \%)$ \\
\hline \hline CDSHW [132] & CERN & $\nu_{\mu} \rightarrow \nu_{\mu}$ & $\approx 1$ & $P_{\mu \mu}>0,95$ \\
\hline E776 [142] & BNL & $\nu_{\mu} \rightarrow \nu_{e}$ & $\approx 1,4$ & $P_{e \mu}<1,5 \times 10^{-3}$ \\
\hline E734 [143] & BNL & $\nu_{\mu} \rightarrow \nu_{e}$ & $\approx 1,2$ & $P_{e \mu}<1,6 \times 10^{-3}$ \\
\hline KARMEN2 [144] & Rutherford & $\bar{\nu}_{\mu} \rightarrow \bar{\nu}_{e}$ & $\sim 0,03$ & $P_{e \mu}<6,5 \times 10^{-4}$ \\
\hline E531 [136] & FNAL & $\nu_{\mu} \rightarrow \nu_{\tau}$ & - & $P_{\mu \tau}<2,5 \times 10^{-3}$ \\
\hline CCFR [133] & FNAL & $\nu_{\mu} \rightarrow \nu_{\tau}$ & $30-600$ & $P_{\mu \tau}<4 \times 10^{-3}$ \\
\hline$[145]$ & & $\nu_{e} \rightarrow \nu_{\tau}$ & $30-600$ & $P_{e \tau}<0,1$ \\
\hline$[146]$ & & $\nu_{\mu} \rightarrow \nu_{e}$ & $30-500$ & $P_{\mu e}<9 \times 10^{-4}$ \\
\hline CHORUS [137] & CERN & $\nu_{\mu} \rightarrow \nu_{\tau}$ & $\approx 27$ & $P_{\mu \tau}<3,4 \times 10^{-4}$ \\
\hline & & $\nu_{e} \rightarrow \nu_{\mu}$ & $\approx 27$ & $P_{e \tau}<2,6 \times 10^{-2}$ \\
\hline NOMAD [140] & CERN & $\nu_{\mu} \rightarrow \nu_{\tau}$ & $\approx 50$ & $P_{\mu \tau}<1,7 \times 10^{-4}$ \\
\hline & & $\nu_{e} \rightarrow \nu_{\tau}$ & $\approx 50$ & $P_{e \tau}<7,5 \times 10^{-3}$ \\
\hline & & $\nu_{\mu} \rightarrow \nu_{e}$ & $\approx 50$ & $P_{\mu e}<6 \times 10^{-4}$ \\
\hline
\end{tabular}

Tabela 3.4: Principais resultados negativos de procura por oscilação em experimentos SBL de neutrinos em aceleradores.

sabor $\bar{\nu}_{\mu} \rightarrow \bar{\nu}_{e}$. A última análise de seus dados, em 2001, apontava um excesso de 87, $9 \pm 22,4 \pm 6$ eventos, correspondendo à uma probabilidade de oscilação de $(2,64 \pm 0,67 \pm 0,45) \times 10^{-3}$.

KARMEN [144] é um calorímetro com camadas de cintilador líquido com excelente resolução em tempo e energia. Eles encontraram um número de eventos consistente com a expectativa de ruído. KARMEN, um ano após LSND, não confirmou os resultados anteriores.

O experimento de MiniBooNE [148], ainda em funcionamento no Fermilab, reutiliza alguns equipamentos do LSND para um feixe de neutrinos com energia 0,5 - 3,0 GeV iniciado por prótons de 8,89 GeV colidindo com um alvo de berílio. Com o auxílio de um sistema magnético, o experimento é capaz de selecionar mésons positivos ou negativos, escolhendo assim dados com neutrinos ou antineutrinos.

O detector de MiniBooNE é uma esfera de 12,2 m de diâmetro preenchida com 800 t de óleo mineral puro. O centro do detector está localizado à uma distância de $541 \mathrm{~m}$ do alvo de berílio. Em sua última análise de dados divulgada em abril de 2007, eles estudaram eventos de neutrinos com energia entre $300<E_{\nu}<$ $3000 \mathrm{MeV}$ [149]. Apesar do espectro apresentar um excesso de $96 \pm 17 \pm 20$ eventos para energias abaixo de $475 \mathrm{MeV}$, nenhum excesso significativo é observado para energias acima deste valor ( $22 \pm 19 \pm 35$ eventos acima do esperado).

Desta forma, a análise conjunta dos três experimentos: LSND, KARMEN e 
MiniBooNE descartam a hipótese de oscilação em duas gerações como explicação para a anomalia encontrada em LSND com $98 \%$ de nível de confiança.

\subsubsection{Experimentos de LBL: K2K, MINOS e OPERA}

Assim como para os neutrinos de reatores, são poucos os experimentos com neutrinos de aceleradores do tipo LBL. No momento, apenas dois deles já divulgaram resultados de suas análises de dados (K2K e MINOS), enquanto um terceiro está em fase final e deverá começar a coletar dados em breve.

\section{K2K e MINOS}

O primeiro experimento de LBL de neutrinos em aceleradores foi o $\mathrm{K} 2 \mathrm{~K}$, proposto em 1997 [150]. Um feixe praticamente puro (98,2\%) de $\nu_{\mu}$ é gerado no acelerador KEK na cidade de Tsukuba no Japão, percorrendo $235 \mathrm{~km}$ até ser detectado em Gifu, no detector de SK.

Este é outro experimento de desaparecimento, em que os neutrinos do múon são inicialmente detectados à 300 m de sua produção por dois "detectores frontais" (DF), e depois medido por SK no denominado "detector remoto" (DR). O DF é formado de um detector Cherenkov de água de $1 \mathrm{kt}$ que é uma miniatura do detector SK.

O feixe de neutrinos do KEK é produzido por prótons de $12 \mathrm{GeV}$ que colidem com um alvo de alumínio. Este alvo de $66 \mathrm{~cm}$ de comprimento é encaixado em dois imãs que servem para focalizar (desfocalizar) os píons positivos (negativos) e káons gerados pelas interações. Os hádrons positivos passam então por um longo túnel de decaimento $(200 \mathrm{~m})$, onde decaem produzindo um feixe de neutrinos de banda larga com uma energia média $\sim 1,4 \mathrm{GeV}$.

O experimento de MINOS já foi descrito anteriormente [134] e possui atualmente uma distância de $730 \mathrm{~km}$ do Fermilab, onde o detector próximo está colocado, até a mina Soudan onde o detector distante se encontra. O feixe de neutrinos do projeto NuMI do Fermilab é capaz de ajustar três regimes de energia: 3, 7 e $15 \mathrm{GeV}$.

Os resultados de ambos os experimentos, K2K e MINOS, observaram um déficit no número de eventos [151, 152, 153]. Em sua última análise, K2K relatou uma observação de 107 eventos enquanto a expectativa na ausência de troca de sabor é $151_{-10}^{+12}$ [152]. MINOS observou um total de 122 eventos abaixo de 10 $\mathrm{GeV}$, enquanto a expectativa sem oscilação é $238 \pm 10,7$ [153]. 


\section{OPERA}

O detector OPERA [154] localizado no subsolo do Laboratório de Gran Sasso (LNGS) na Itália foi desenvolvido para executar a primeira detecção de neutrinos no modo de aparecimento, através da observação de $\nu_{\tau}$. Ele está colocado no feixe LBL de alta energia entre o CERN e LNGS, com uma distância entre a fonte e o detector de $730 \mathrm{~km}$. Em agosto de 2006 uma primeira tomada de dados foi realizada com sucesso [155].

\subsubsection{Futuros experimentos: superfeixes de neutrinos}

A próxima geração de experimentos com neutrinos de aceleradores deverá fazer uso de superfeixes de neutrinos. Esses superfeixes são definidos como os convencionais, porém produzidos por instalações com potências na escala de megawatts. Os dois experimentos mais próximos são: T2K, no Japão [90] e NuMI-OA, no Fermilab [76, 156].

O T2K (Tokai to Kamioka) se utiliza da nova instalação de $0.8 \mathrm{MW}$ em JAERI, J-Parc com energia inicial dos prótons de $40 \mathrm{GeV}$. Um FFE permitirá que os neutrinos de T2K alcancem energias entre 500 e $700 \mathrm{MeV}$. A distância percorrida será de $295 \mathrm{~km}$ (LBL), até o detector de SK.

No Fermilab, enquanto produz feixes para o experimento de MINOS, NuMI também produz neutrinos de FFE. Por exemplo, um detector colocado à $20 \mathrm{mrad}$ da linha do feixe de NuMI veria um feixe de neutrinos estreitamente acentuado em $2 \mathrm{GeV}$. A distância percorrida poderia estar entre 700 e $900 \mathrm{~km}$. Usando esta idéia, o conceito de $\mathrm{NO} \nu \mathrm{A}$ [76] foi proposto utilizando um calorímetro baseado em cintilador líquido. Existe também uma proposta de se utilizar uma câmara de projeção de argônio líquido como detector, FLARE [156].

Numa próxima geração de experimentos, poderão ser empregados feixes $\beta$ [157] ou Fábricas de Neutrinos [158], cujos neutrinos teriam energias, respectivamente, $0,2-5 \mathrm{GeV}$ e $7-50 \mathrm{GeV}$, e as distâncias percorridas estariam entre algumas centenas e $8000 \mathrm{~km}$.

\subsubsection{Resumo dos resultados}

\begin{tabular}{|c|c|}
\hline Experimento & Resultado: Medido/MP \\
\hline \hline SBL & consistentes com a unidade \\
\hline LSND & déficit não confirmado \\
\hline K2K & $0,71_{-0,09}^{+0,06}$ \\
\hline MINOS & $0,51 \pm 0,1$ \\
\hline
\end{tabular}

Tabela 3.5: Resumo dos resultados de oscilação de neutrinos em aceleradores [109]. 


\section{Capítulo 4}

\section{Análise dos parâmetros de oscilação}

No capítulo anterior foram apresentados todos os resultados de experimentos de neutrinos que evidenciaram a oscilação no setor. Durante a exposição foi ficando cada vez mais forte a necessidade de algum mecanismo de oscilação de sabor para explicação dos dados experimentais.

Neste capítulo introduzimos a idéia de oscilação da maneira convencional para podermos aplicar os resultados teóricos na análise dos dados experimentais. Na segunda parte, os valores dos parâmetros que melhor ajustam a oscilação são discutidos.

\subsection{Oscilação de neutrinos}

A primeira parte desta seção é consagrada à uma breve discussão sobre a maneira habitual de se introduzir à oscilação de neutrinos, que por não ser completamente rigorosa, não poderia ser apresentada sem explicações.

Nas seções seguintes são discutidos os métodos de interpretação dos resultados de neutrinos com mecanismos de oscilação no vácuo e na matéria.

\subsubsection{Abordagem convencional}

A abordagem convencional do estudo de oscilação de neutrinos vem da extensão natural de oscilação no setor de quarks, onde emprega-se a MQ clássica. O formalismo matemático da MQ para um sistema qualquer com um número finito de graus de liberdade é bem estabelecido e relativamente simples de se usar.

A noção fundamental que necessitamos da MQ é a adoção de uma hamiltoniana para descrever o sistema e a utilização de ferramentas para encontrar a solução de estados estacionários. Para aplicar este formalismo é necessário portanto postular a forma desta hamiltoniana. 
Isto é consideravelmente simples no caso de oscilação de neutrinos induzida por massa, onde os autoestados de massa são considerados diferentes (combinações lineares) dos estados que participam das interações, chamados também de autoestados de sabor, família ou geração.

O formalismo se torna mais ambíguo quando efeitos de matéria (que ocorre quando os neutrinos atravessam um meio material) devem ser levados em consideração. Um Ansatz, proposto por Wolfenstein, foi utilizado por algum tempo na falta de uma demonstração mais precisa da forma do potencial. Obviamente, os físicos sempre estiveram cientes de que uma demonstração mais rigorosa, baseada no MP, deveria existir. Contudo, isso não coloca problemas para que uma primeira introdução possa ser feita de maneira simples e intuitiva, sendo assim convencionalmente adotada.

Um exemplo dessa metodologia convencional é a "função delta de Dirac", cuja primeira abordagem é sempre dada com enfoque prático e sobre a noção de funções. Somente anos mais tarde, o conceito de distribuições (criado por Schwartz) aparece e então o rigor é retomado. Outro exemplo, mais atual são os cursos introdutórios de Teoria Quântica de Campos. Estes costumam ser lecionados com o formalismo de integrais de trajetórias de Feynman, as quais não possuem medidas bem definidas no sentido matemático.

Portanto, acreditamos que uma primeira abordagem concisa possa ser feita para chegar rapidamente aos resultados necessários, e num próximo capítulo daremos uma explicação mais detalhada.

\subsubsection{Oscilação de neutrinos monoenergéticos}

Considerando apenas neutrinos monoenergéticos, se eles possuem massa, seus autoestados de massa podem diferir dos autoestados de interação. Uma matriz de mistura, $U$, deve ser introduzida na combinação linear

$$
\left|\nu_{\alpha}\right\rangle=\sum_{i=1}^{3} U_{\alpha j}\left|\nu_{j}\right\rangle
$$

em que $\left|\nu_{\alpha}\right\rangle$ são os componentes da base de autoestados de sabor, $\alpha=e, \mu, \tau$, e $\left|\nu_{j}\right\rangle$ os componentes da base de autoestados de massa, $j=1,2$, 3. A matriz de mistura depende efetivamente de três ângulos $\left(\theta_{12}, \theta_{13}, \theta_{23}\right.$, que misturam as famílias) e uma fase ( $\delta$, que dá origem à violação da simetria $\left.\mathrm{CP}^{1}\right)$.

Utilizando os limites superiores de massa dos neutrinos, estas são consideradas partículas relativísticas na grande maioria dos casos, pois $E \gg m_{\nu}$. A

\footnotetext{
${ }^{1} \mathrm{~A}$ violação de $\mathrm{CP}$ implica em diferentes probabilidades para neutrinos e antineutrinos oscilarem: $P\left(\nu_{\alpha} \rightarrow \nu_{\beta}\right) \nrightarrow P\left(\bar{\nu}_{\alpha} \rightarrow \bar{\nu}_{\beta}\right)$.
} 
relação relativística entre energia e momento é $E_{j}=\sqrt{p^{2}+m_{j}^{2}}$, e utilizando a aproximação usual para pequenas massas, $E_{j} \simeq|p|+\frac{m_{j}^{2}}{2|p|}$, chega-se facilmente a equação de evolução no vácuo $i \frac{d}{d t}\left|\nu_{j}\right\rangle=\left[p+\frac{1}{2 p} m_{j}^{2}\right]\left|\nu_{j}\right\rangle$. Os autoestados dessa equação são os próprios autoestados de massa, por isso às vezes são chamados de autoestados do vácuo, para não confundir com os autoestados de massa que existem num meio material.

Como as partículas são sempre criadas e detectadas nos autoestados de interação, a equação que nos interessa deve estar nesta outra base. Com a substituição $\left|\nu_{\alpha}\right\rangle=U_{\alpha j}\left|\nu_{j}\right\rangle$, obtém-se $i \frac{d}{d t}\left|\nu_{\beta}\right\rangle=\left[p+\frac{1}{2 p} U_{\beta j} m_{j}^{2} U_{j \alpha}^{\dagger}\right]\left|\nu_{\alpha}\right\rangle$. Já que o resultado da probabilidade de transição não depende de termos proporcionais à identidade na hamiltoniana, pode-se subtrair o valor $p+\frac{m_{1}^{2}}{2 p}$ e obter finalmente a forma tradicional da equação de evolução de neutrinos no vácuo:

$$
i \frac{d}{d x}\left(\begin{array}{c}
\nu_{e} \\
\nu_{\mu} \\
\nu_{\tau}
\end{array}\right)=\frac{1}{2 E} U\left(\begin{array}{ccc}
0 & 0 & 0 \\
0 & \Delta m_{21}^{2} & 0 \\
0 & 0 & \Delta m_{31}^{2}
\end{array}\right) U^{\dagger}\left(\begin{array}{c}
\nu_{e} \\
\nu_{\mu} \\
\nu_{\tau}
\end{array}\right),
$$

cujas soluções são da forma $P=P\left(\frac{\Delta m_{21}^{2}}{E} x, \frac{\Delta m_{31}^{2}}{E} x, \theta_{12}, \theta_{13}, \theta_{23}, \delta\right)$, e que apesar de extensas, são analiticamente simples ${ }^{2}$. Neste último passo é utilizada a aproximação relativística $x \simeq t$. Desta forma, pode-se observar que as probabilidades dependem apenas dos valores das diferenças de massas ao quadrado, e mais que isso, da quantidade $\frac{\Delta m_{i j}^{2}}{E} x$ com $\Delta m_{i j}^{2}=m_{i}^{2}-m_{j}^{2}$. Portanto, o valor $L_{i j}=\frac{4 \pi E}{\Delta m_{i j}^{2}}$ recebe o nome de comprimento de oscilação, pois fornece a distância mínima em que o sistema retorna ao estado original.

\subsubsection{Fórmulas em duas gerações}

Apoiado nos estudos de oscilação no setor de quarks, onde os káons produzidos $K^{0}$ e $\bar{K}^{0}$ são combinações lineares dos estados físicos $K_{L}$ e $K_{S}$, uma análise em duas gerações é sugerida. Isto simplifica muito mais o estudo e compreensão das oscilações no setor de neutrinos.

Neste cenário, apenas uma diferença de massa $\Delta m^{2}$ e um ângulo $\theta$ estão presentes. Para generalizar a aplicação, as contas são feitas com dois estados de interação $\nu_{X}$ e $\nu_{Y}$, que representam os autoestados físicos de interação ou combinações lineares deles. A equação se torna, após alguma manipulação,

$$
i \frac{d}{d x}\left(\begin{array}{c}
\nu_{X} \\
\nu_{Y}
\end{array}\right)=\frac{\Delta m^{2}}{4 E}\left(\begin{array}{cc}
-\cos 2 \theta & \sin 2 \theta \\
\sin 2 \theta & \cos 2 \theta
\end{array}\right)\left(\begin{array}{c}
\nu_{X} \\
\nu_{Y}
\end{array}\right),
$$

\footnotetext{
${ }^{2}$ Todos os resultados completos que aqui não são apresentados podem ser encontrados nas Ref. [159, 160].
} 
cujas soluções para as probabilidades de conversão de sabor e sobrevivência são respectivamente

$$
\begin{gathered}
P_{X Y}(x)=\sin ^{2} 2 \theta \sin ^{2}\left(\frac{\Delta m^{2}}{4 E} x\right) \\
P_{X X}(x)=1-\sin ^{2} 2 \theta \sin ^{2}\left(\frac{\Delta m^{2}}{4 E} x\right),
\end{gathered}
$$

em que para fazer aplicação numérica (retornando os valores de $\hbar$ e $c$ ao problema), o argumento é $\frac{\Delta m^{2}}{4 E} x=1,27\left(\frac{\Delta m^{2}}{\mathrm{eV}^{2}}\right)\left(\frac{x}{\mathrm{~m}}\right)\left(\frac{\mathrm{MeV}}{E}\right)$.

Das soluções na Eq. (4.4) vemos que o espaço de parâmetros é coberto com $\Delta m^{2} \geq 0$ e $0 \leq \theta \leq \pi / 2$ (ou de maneira alternativa por $0 \leq \theta \leq \pi / 4$ e ambos os sinais de $\Delta m^{2}$ ). Na mudança do sinal de massa, $\Delta m^{2} \rightarrow-\Delta m^{2}$, e do octante do ângulo de mistura, $\theta \rightarrow \frac{\pi}{2}-\theta$, leva à redefinição dos autoestados de massa $\nu_{1} \leftrightarrow \nu_{2}$. Como visto na Eq. (4.4), $P_{X Y}$ é de fato invariante por tais transformações separadamente. Isto implica que existe uma ambigüidade na interpretação de $P_{X Y}$ em termos da mistura de dois neutrinos: os dois conjuntos de parâmetros, $\left(\Delta m^{2}, \theta\right)$ e $\left(\Delta m^{2}, \frac{\pi}{2}-\theta\right)$, resultam na mesma probabilidade de transição no vácuo.

\subsubsection{Efeitos com distribuição energética}

Para entender melhor a natureza dos resultados, devemos levar em consideração que as fontes de neutrinos não são monoenergéticas. Isto ocorre não apenas por causa das relações de incerteza, mas sobretudo devido à distribuição energética que existe num feixe físico.

Essa distribuição pode ser representada por uma função $\Phi(E)$ que engloba a resolução do detector, as seções de choque, o espectro do fluxo e outros fatores de um determinado experimento, como por exemplo a eficiência de detecção. Normalmente esta função pode ser escrita da seguinte forma,

$$
\Phi(E)=\frac{\frac{d \phi(E)}{d E} \sigma(E) R(E)}{\int d E^{\prime} \frac{d \phi\left(E^{\prime}\right)}{d E^{\prime}} \sigma\left(E^{\prime}\right) R\left(E^{\prime}\right)},
$$

onde $\frac{d \phi(E)}{d E}$ é o espectro de energia de neutrinos, $\sigma$ a seção de choque do processo de detecção, e $R(E)$ a resolução do detector.

A probabilidade de conversão média obtida num experimento real é então dada por

$$
\left\langle P_{X Y}(x)\right\rangle=\sin ^{2} 2 \theta \int d E \Phi(E) \sin ^{2}\left(\frac{\Delta m^{2}}{4 E} x\right)
$$

num canal de oscilação entre dois sabores quaisquer.

Supondo agora que num experimento o número de eventos observados foi $N_{o b s}$, menor ou igual à previsão teórica sem oscilação, $N_{t e o}$. Então a taxa $P_{0} \equiv$ 


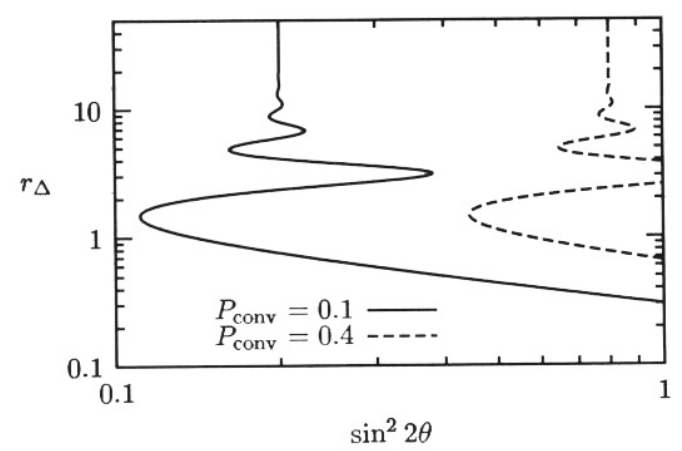

Figura 4.1: Contornos de probabilidades de conversão iguais no vácuo para dois valores diferentes, 0,1 e 0,4 .

$1-\frac{N_{o b s}}{N_{t e o}}$ fornece a probabilidade de conversão obtida. Para analisar os dados pela interpretação de oscilação fazemos $\left\langle P_{X Y}\right\rangle=P_{0}$. É conveniente definir a razão $r_{\Delta}=\frac{\Delta m^{2}}{4\langle E\rangle} x$ para entender o significado de $P_{0}$. Assim, a Eq. (4.6) pode se escrever de duas formas

$$
\begin{aligned}
P_{0} & =\sin ^{2} 2 \theta \int d E \Phi(E) \sin ^{2}\left(r_{\Delta}\langle E\rangle / E\right) \\
& =\frac{1}{2} \sin ^{2} 2 \theta \int d E \Phi(E)\left[1-\cos \left(2 r_{\Delta}\langle E\rangle / E\right)\right] .
\end{aligned}
$$

Se $r_{\Delta} \gg 1$,olhamos para a segunda igualdade, Eq. (4.8), onde o termo do cosseno terá grandes flutuações e terá média zero pelo lema de Schwartz. Isto significa que $\sin ^{2} 2 \theta=2 P_{0}$. Portanto, no gráfico de $r_{\Delta} \times \sin ^{2} 2 \theta$, o contorno de igual probabilidade de conversão é vertical, como visto na Figura 4.1.

Em contrapartida, se $r_{\Delta} \ll 1$, a primeira igualdade, Eq. (4.7), pode ser aproximada com a troca do seno pelo argumento, obtendo $r_{\Delta}^{2} \sin ^{2} 2 \theta=\frac{P_{0}}{\langle E\rangle^{2}\left\langle 1 / E^{2}\right\rangle}$. Como o lado direito dessa igualdade é constante num dado experimento, isto mostra que no gráfico logarítmico a parte mais baixa se aproxima de uma reta com coeficiente angular de $-1 / 2$, como visto na Figura 4.1. O comportamento intermediário da curva depende das características do experimento, principalmente da resolução do detector.

A análise de neutrinos se propagando no vácuo em duas gerações é simples. De fato, um experimento com resultado negativo para oscilação, ou seja, se a probabilidade de conversão é apenas menor que um certo valor, por exemplo 0,1 , no cenário de duas famílias o canto superior direito da linha cheia na Figura 4.1 fica descartado. Caso contrário, se existe uma certa probabilidade de transição encontrada com valores fixos para $\langle E\rangle$ e $x$, uma região intermediária é aceita, por exemplo entre as curvas correspondentes à 0,1 e 0,4 da Figura 4.1.

Pela ambigüidade observada no final da seção anterior, a Figura 4.1 deve ser 
simétrica, ou ter a imagem refletida, pela reta $\theta=\frac{\pi}{4}$.

\subsubsection{Efeitos de matéria}

Quando a trajetória dos neutrinos passa por um meio que não o vácuo ${ }^{3}$, deve-se incluir na equação de evolução um potencial correspondente às interações dos neutrinos com a matéria [37, 38].

Os meios materiais tradicionais não contém múons ou taus em seu interior, somente elétrons. Sendo assim, as interações de NC do MP serão as mesmas para todos os sabores de neutrinos, adicionando um termo que é proporcional à identidade na hamiltoniana. Por ocasionar apenas uma fase ao resultado final, não consideramos este potencial. Já as interações de CC ocorrem apenas aos neutrinos eletrônicos, e devem ser proporcionais à quantidade de elétrons do meio. Desta forma, o Ansatz proposto por Wolfenstein ${ }^{4}$ [38] foi

$$
V(x)=\sqrt{2} G_{F} N_{e}(x) \simeq 7,6 Y_{e} \frac{\rho}{10^{14} \mathrm{~g} / \mathrm{cm}^{3}} \mathrm{eV},
$$

onde $N_{e}$ é a densidade de elétrons, $Y_{e}=\frac{N_{e}}{N_{p}+N_{n}}$ a densidade relativa entre elétrons e núcleons, e $\rho$ a densidade da matéria. Os valores típicos são: no núcleo terrestre $\rho \sim 10 \mathrm{~g} / \mathrm{cm}^{3}$ e $V \sim 10^{-13} \mathrm{eV}$; no interior solar $\rho \sim 100 \mathrm{~g} / \mathrm{cm}^{3}$ e $V \sim 10^{-12} \mathrm{eV}$; e próximo ao centro de uma supernova $\rho \sim 10^{14} \mathrm{~g} / \mathrm{cm}^{3}$ e $V \sim 1 \mathrm{eV}$.

A equação de evolução com o potencial de matéria se torna

$$
i \frac{d}{d x}\left(\begin{array}{c}
\nu_{e} \\
\nu_{\mu} \\
\nu_{\tau}
\end{array}\right)=\left[U\left(\begin{array}{ccc}
0 & 0 & 0 \\
0 & \frac{\Delta m_{21}^{2}}{2 E} & 0 \\
0 & 0 & \frac{\Delta m_{31}^{2}}{2 E}
\end{array}\right) U^{\dagger}+V(x)\left(\begin{array}{ccc}
1 & 0 & 0 \\
0 & 0 & 0 \\
0 & 0 & 0
\end{array}\right)\right]\left(\begin{array}{c}
\nu_{e} \\
\nu_{\mu} \\
\nu_{\tau}
\end{array}\right),
$$

cujo estudo de soluções exatas e aproximadas formam o tema central do presente projeto de mestrado, e será discutido em detalhers nos Capítulos 6,7 e 8 .

Contudo, análogo ao caso do vácuo, podemos estudar os efeitos em duas gerações. Neste caso a equação se reduz ${ }^{5}$ à

$$
i \frac{d}{d x}\left(\begin{array}{c}
\nu_{e} \\
\nu_{Y}
\end{array}\right)=\frac{\Delta m^{2}}{4 E}\left(\begin{array}{cc}
-\cos 2 \theta+A(x) & \sin 2 \theta \\
\sin 2 \theta & \cos 2 \theta-A(x)
\end{array}\right)\left(\begin{array}{c}
\nu_{X} \\
\nu_{Y}
\end{array}\right)
$$

onde $A(x)=\frac{2 E V(x)}{\Delta m^{2}}$. É interessante notar que os autoestados de massa na matéria

\footnotetext{
${ }^{3}$ Os casos reais considerados são o Sol, a Terra e supernovas.

${ }^{4}$ Corrigido mais tarde por Bethe [161].

${ }^{5}$ Basta adicionar $-\left(p+\frac{m_{1}^{2}+m_{2}^{2}}{4 E}\right)-V(x) / 2$ à equação original.
} 
dependem da posição, e que a Eq. (4.11) na base desses autoestados é

$$
i \frac{d}{d x}\left(\begin{array}{c}
\tilde{\nu}_{1} \\
\tilde{\nu}_{2}
\end{array}\right)=\left(\begin{array}{cc}
\Delta(x) & -i \dot{\tilde{\theta}}(x) \\
i \dot{\tilde{\theta}}(x) & -\Delta(x)
\end{array}\right)\left(\begin{array}{c}
\tilde{\nu}_{1} \\
\tilde{\nu}_{2}
\end{array}\right)
$$

onde $\Delta(x)=\frac{\Delta m^{2}}{4 E} \sqrt{(\cos 2 \theta-A(x))^{2}+\sin ^{2} 2 \theta}$ é o autovalor na posição $x$, e $\dot{\tilde{\theta}}=\frac{\left(\Delta m^{2}\right)^{2} \sin 2 \theta}{2 \Delta(x)^{2}} \dot{A}$ é a variação do ângulo de mistura efetivo na matéria, proporcional à variação do potencial. Portanto, diferente do caso no vácuo, os autoestados de massa não evoluem independentemente, mas possuem um termo fora da diagonal. Assim como na base de sabor, e em vários problemas na MQ, o termo fora da diagonal é responsável por uma probabilidade de transição dos autoestados (cruzamento). A matriz não diagonal é proporcional à matriz de Pauli $\sigma_{2}$, desta forma as probabilidades de cruzamento de nível em questão são chamadas de Landau-Zener [162, 163]. Independentes de Stueckelberg [164], Landau e Zener foram os primeiros à estudarem tais probabilidades no estudo de colisões atômicas. Na época, eles se interessavam pelo problema aproximado com potencial linear, cuja solução obtida aplicada ao problema de neutrinos é $P_{L Z}=e^{-\frac{\pi}{2} \gamma}$ [165], em que $\gamma=\frac{\Delta m^{2} \sin ^{2} 2 \theta}{2 E \cos 2 \theta|d / d x \ln V(x)| \text { res }}$ é o chamado parâmetro de adiabaticidade, cujo significado veremos mais abaixo. Esta não é a melhor fórmula para todo tipo de potencial, sendo assim veremos no Capítulo 8 um método para a obtenção de fórmulas mais precisas e o Ansatz proposto por Kuo e Pantaleone [160].

A maneira mais simples de se resolver a Eq. (4.11) é através da aproximação adiabática. Como o nome já diz, o método é uma aproximação válida apenas no caso de um potencial que varia lentamente, mais precisamente a condição de validade do resultado é $\gamma \gg 1$. Isto conduz à desprezar os termos fora da diagonal na Eq. (4.12), e o procedimento de resolução se torna evidente, já que a equação é linear e a condição $[H(x), H(y)]=0$ é satisfeita. Além disso, os termos oscilantes podem ser desconsiderados já que existem muitas oscilações para uma variação lenta nas quantidades. Classicamente, o cálculo da probabilidade se torna

$$
\begin{aligned}
P_{e e} & =\left(\begin{array}{ll}
1 & 0
\end{array}\right)\left(\begin{array}{cc}
\cos ^{2} \theta & \sin ^{2} \theta \\
\sin ^{2} \theta & \cos ^{2} \theta
\end{array}\right)\left(\begin{array}{cc}
\cos ^{2} \tilde{\theta} & \sin ^{2} \tilde{\theta} \\
\sin ^{2} \tilde{\theta} & \cos ^{2} \tilde{\theta}
\end{array}\right)\left(\begin{array}{l}
1 \\
0
\end{array}\right) \\
& =\frac{1}{2}(1+\cos 2 \theta \cos 2 \tilde{\theta}),
\end{aligned}
$$

onde foi usado o ângulo efetivo na matéria, definido por

$$
\sin 2 \tilde{\theta}=\frac{\sin 2 \theta}{\sqrt{(\cos 2 \theta-A(x))^{2}+\sin ^{2} 2 \theta}} .
$$




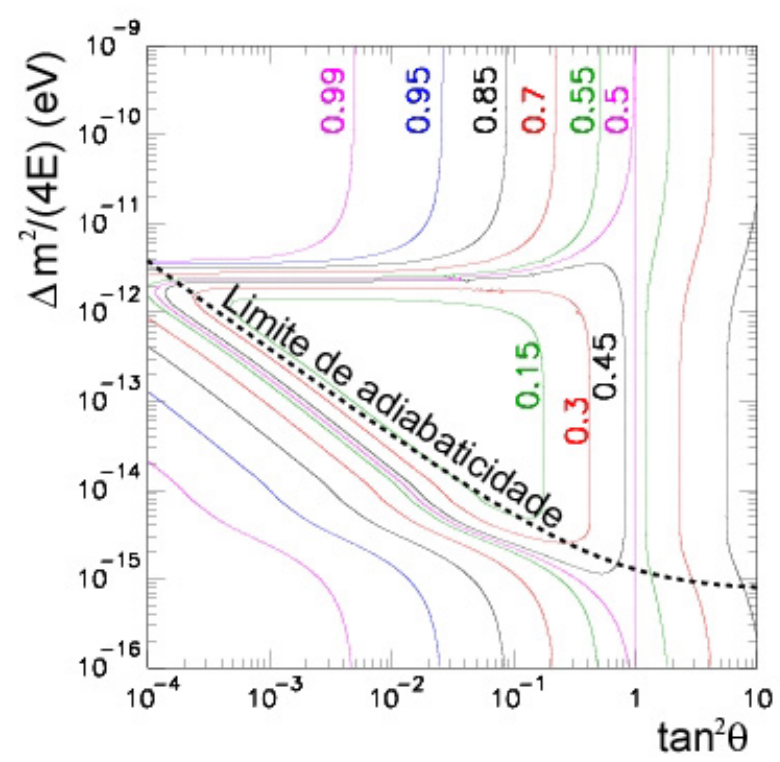

Figura 4.2: Isocontornos de mesma probabilidade de sobrevivência $P_{e e}$ no Sol. O limite de aplicabilidade da aproximação adiabática está representado pelas linhas tracejadas.

Quando trabalhamos numa região em que a aproximação de adiabaticidade não pode ser utilizada, costuma-se utilizar uma correção à solução através da introdução das probabilidades de cruzamento de nível $P_{L Z}$. Neste caso, a solução pode ser escrita da seguinte forma

$$
\begin{aligned}
P_{e e}= & \left(\begin{array}{ll}
1 & 0
\end{array}\right)\left(\begin{array}{cc}
\cos ^{2} \theta & \sin ^{2} \theta \\
\sin ^{2} \theta & \cos ^{2} \theta
\end{array}\right)\left(\begin{array}{cc}
1-P_{L Z} & P_{L Z} \\
P_{L Z} & 1-P_{L Z}
\end{array}\right) \times \\
& \times\left(\begin{array}{cc}
\cos ^{2} \tilde{\theta} & \sin ^{2} \tilde{\theta} \\
\sin ^{2} \tilde{\theta} & \cos ^{2} \tilde{\theta}
\end{array}\right)\left(\begin{array}{c}
1 \\
0
\end{array}\right) \\
= & \frac{1}{2}+\left(\frac{1}{2}-P_{L Z}\right) \cos 2 \theta \cos 2 \tilde{\theta} .
\end{aligned}
$$

A análise no caso de neutrinos que atravessam um meio material não é tão simples, mas a Figura 4.2 representa a curva obtida para $\gamma \simeq 1$. A aproximação adiabática não pode ser aplicada à esquerda da linha tracejada, neste caso utilizamos a correção pelo método de Landau-Zener. Podemos observar também que a simetria em torno de $\theta=\pi / 4$ é perdida pela adição do efeito de matéria.

\subsection{Determinação dos parâmetros de oscilação}

Nesta parte nos ocupamos de combinar os resultados dos experimentos discutidos na capítulo anterior para verificar se a hipótese de oscilação no setor de neutrinos é consistente. Em seguida iremos discutir quais os valores mais prováveis para os parâmetros de oscilação. 
Nas análises gerais verificamos a consistência da hipótese de oscilação como provável explicação para os resultados experimentais (com excessão de LSND), além de apresentarmos os argumentos para analisar os resultados no contexto simplificado de duas gerações.

Nas análises precisas, utilizamos as idéias apresentadas na seção 4.1, sobretudo o fato que para neutrinos se propagando no vácuo devemos obter um resultado semelhante ao da Figura 4.1, enquanto a propagação num meio material fornece dados semelhante ao da Figura 4.2, com suas regiões permitidas ou proibidas. Se ao invés disso os dados forem tomados para vários valores de $x$ e $\langle E\rangle$ num mesmo experimento, a região correspondente pode ser mais precisa e informações mais diretas podem ser tiradas.

\subsubsection{Análises gerais}

As fontes mais confiáveis de neutrinos são as humanas, pois tem-se de alguma forma controle sobre sua produção, reforçado pelos resultados negativos para oscilação de experimentos de SBL. Sendo assim, a primeira análise recai sobre neutrinos de reatores e aceleradores.

A escala de massa investigada por um determinado experimento é $\gtrsim\langle E\rangle / L$, afinal como foi visto $L=4 \pi E / \Delta m^{2}$ é o comprimento de oscilação. As principais escalas investigadas nos experimentos são apresentadas na Tabela 4.1.

\begin{tabular}{|c|c|c|c|}
\hline Experimentos & $\Delta m^{2}\left(\mathrm{eV}^{2}\right)$ & Canal & Resultado \\
\hline \hline reatores SBL & $\gtrsim 2 \times 10^{-2}$ & $\bar{\nu}_{e} \rightarrow \bar{\nu}_{e}$ & concorda \\
\hline CHOOZ e Palo Verde & $\gtrsim 2 \times 10^{-3}$ & $\bar{\nu}_{e} \rightarrow \bar{\nu}_{e}$ & concorda \\
\hline KamLAND & $\gtrsim 1,5 \times 10^{-5}$ & $\bar{\nu}_{e} \rightarrow \bar{\nu}_{e}$ & desaparece \\
\hline K2K e MINOS & $\gtrsim 10^{-3}$ & $\nu_{\mu} \rightarrow \nu_{\mu}$ e $\nu_{\mu} \rightarrow \nu_{e}$ & desaparece \\
\hline LSND e MiniBOONE & $\gtrsim 2 \times 10^{-1}$ & $\bar{\nu}_{\mu} \rightarrow \bar{\nu}_{e}$ e $\nu_{\mu} \rightarrow \nu_{e}$ & não confirmado \\
\hline
\end{tabular}

Tabela 4.1: Análise geral dos principais resultados obtidos em experimentos com neutrinos produzidos pelo homem. Dizemos que o resultado concorda se a previsão teórica sem oscilação é compatível com o número de eventos observados e desaparece caso haja um déficit no número de eventos medidos.

Portanto as seguintes observações podem ser consideradas:

1. CHOOZ e Palo Verde mostram que ou os ângulos que misturam a primeira família $\left(\theta_{12}\right.$ e $\left.\theta_{13}\right)$ são pequenos, ou se algum ângulo é grande, a escala de massa envolvida é $\ll 10^{-3} \mathrm{eV}^{2}$;

2. K2K e MINOS mostram que a segunda família está envolvida numa oscilação com escala de massa $\Delta M^{2} \sim 10^{-3} \mathrm{eV}^{2}$ e um ângulo grande que não envolve a primeira família, portanto $\theta_{23} \approx 45^{\circ}$. 
3. KamLAND mostra que a primeira família está envolvida numa oscilação cuja escala de massa é $\Delta m^{2} \sim 10^{-5} \mathrm{eV}^{2}$ com um ângulo de mistura grande $\left(\theta_{12}\right.$ ou $\left.\theta_{13}\right)$;

4. LSND indica que existe uma terceira escala de massa $\Delta \mu^{2} \sim 1 \mathrm{eV}^{2}$, o que seria inconsistente com a hipótese de oscilação com três famílias, já que esta só possibilita a existência de duas diferenças de massa independentes. Como seu resultado não foi confirmado por MiniBooNE, ainda buscamos compreender esse resultado.

Conclui-se portanto que existem duas escalas distintas envolvidas nas diferenças de massas, de forma que $\Delta m^{2} / \Delta M^{2} \ll 1$. A escala de massa grande, $\Delta M^{2} \sim$ $10^{-3} \mathrm{eV}^{2}$, não influencia o resultado de CHOOZ e Palo Verde, implicando num ângulo de mistura pequeno entre a primeira família e essa escala de massa, $\theta_{13} \approx$ 0. KamLAND aponta para a existência de um ângulo grande envolvendo a primeira famíla, só sobra $\theta_{12}$, e uma escala de massa $\Delta m^{2} \sim 10^{-5} \mathrm{eV}^{2}$.

\begin{tabular}{|c|c|c|c|c|}
\hline Experimento & $x(\mathrm{~km})$ & $E(\mathrm{GeV})$ & $\Delta m^{2}\left(\mathrm{eV}^{2}\right)$ & canal \\
\hline \hline solar & $10^{8}$ & $10^{-4}-10^{-2}$ & $\gtrsim 10^{-12}$ & $\nu_{e} \rightarrow \nu_{\mu, \tau}$ \\
\hline atmosférico & $10^{2}-10^{4}$ & $10^{-1}-10^{2}$ & $\gtrsim 10^{-5}$ & $\nu_{\mu} \rightarrow \nu_{\tau}$ \\
\hline
\end{tabular}

Tabela 4.2: Escala de massa investigada com neutrinos de fontes naturais. A sensibilidade é estimada para oscilação no vácuo.

As escalas de massas envolvidas em experimentos com neutrinos de fontes naturais são resumidas na Tabela 4.2. Vê-se facilmente que as conclusões tomadas acima não contradizem os experimentos, apesar de uma sutileza que envolverá os neutrinos solares quando feita as análises mais precisas. Desta forma colocamos:

$$
\begin{array}{cccc}
\Delta m_{\odot}^{2} & =\Delta m_{21}^{2}=\Delta m^{2} \sim 10^{-5} \mathrm{eV}^{2} & \text { e } & \theta_{\odot}=\theta_{12} \\
\Delta m_{\mathrm{atm}}^{2} & =\Delta m_{31}^{2}=\Delta M^{2} \sim 10^{-3} \mathrm{eV}^{2} & \text { e } & \theta_{\mathrm{atm}}=\theta_{23} .
\end{array}
$$

\subsubsection{Análises precisas}

Nesta última seção apresentamos os melhores ajustes para os valores dos parâmetros de oscilação.

As análises feitas empregam os resultados em duas gerações utilizando as aproximações $\Delta m_{21}^{2} / \Delta m_{31}^{2} \ll 1 \mathrm{e} /$ ou $\theta_{13} \rightarrow 0$, obtidas das análises gerais feitas aos parâmetros na seção anterior. 
Setor com pequena escala de massa: $\Delta m_{21}^{2} \sim 10^{-5} \mathrm{eV}^{2}$

A explicação usual para os dados solares de neutrinos descritos no terceiro capítulo é a oscilação de $\nu_{e}$ para uma combinação de $\nu_{\mu}$ ou $\nu_{\tau}$, ou para um neutrino estéril ${ }^{6} \nu_{s}$. Contudo, as medidas de $\mathrm{NC}$ de SNO descartam a última possibilidade com certeza de mais de $7-\sigma$, já que no caso de oscilação $\nu_{e} \rightarrow \nu_{s}$ as medidas CC e NC de SNO deveriam ser iguais.

A análise somente dos resultados de neutrinos solares faz uso do efeito de matéria para acomodar todos os valores na explicação por oscilação induzida por massa descrita acima. Apesar disso, sem os dados de KamLAND, existiam ainda quatro regimes de oscilação compatíveis dentro dos erros com os dados experimentais. Estes eram denotados: VAC - oscilação apenas no vácuo; SMA - na matéria com ângulo pequeno; LMA - na matéria com ângulo grande; e LOW - na matéria e massa pequena. Sob a suposição da validade de CPT ser conservada, as medidas em KamLAND apontaram LMA como a única solução capaz de acomodar a oscilação vista neste experimento de reator.

Os resultados das análises feitas com experimentos solares (incluindo efeito de matéria) e KamLAND estão mostradas na Figura 4.3. Uma descrição detalhada de incluir todas as incertezas e correlações nesta análise pode ser encontrada na Ref. [166] e referências inclusas.
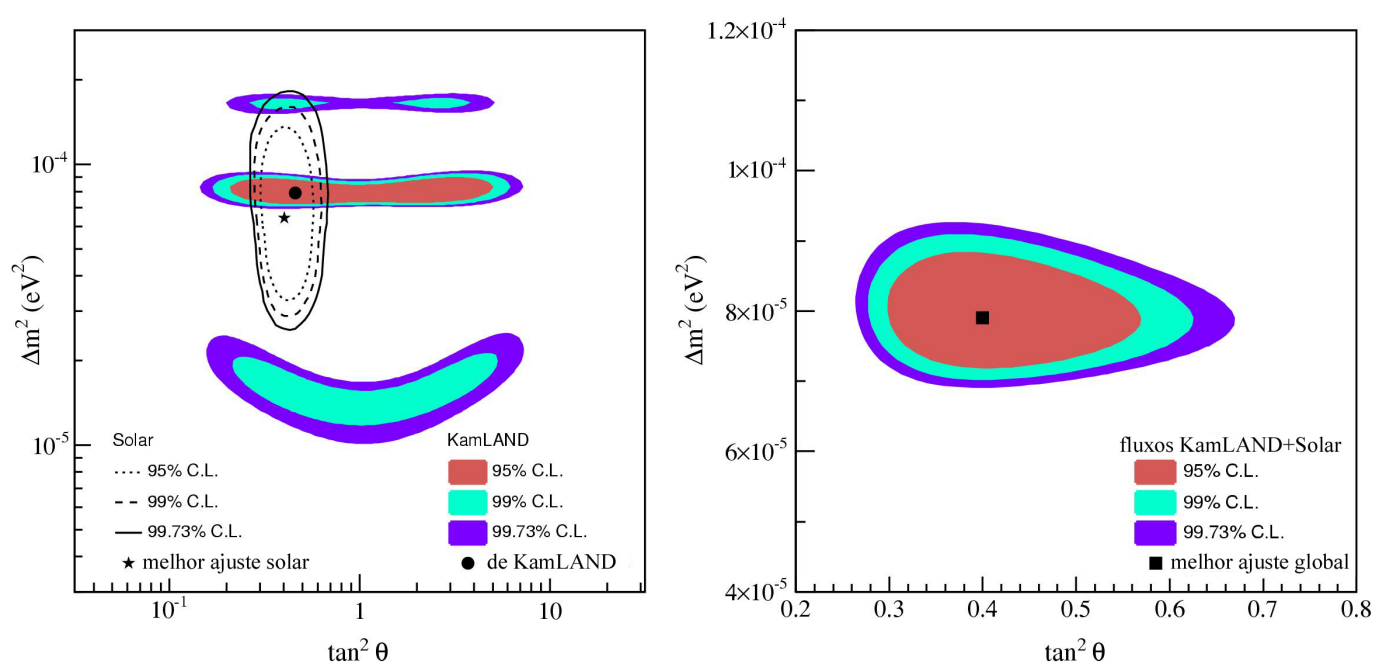

Figura 4.3: O gráfico da esquerda apresenta a região permitida dos parâmetros de oscilação dos dados de antineutrinos de KamLAND (regiões preenchidas) e de experimentos de neutrinos solares (linhas). Na direita, o gráfico mostra o resultado combinado da análise em duas gerações dos fluxos de neutrinos solares e KamLAND assumindo a invariância CPT. O ajuste fornece $\Delta m_{21}^{2}=7,9_{-0,5}^{+0,6} \times 10^{-5} \mathrm{eV}^{2}$ e $\tan ^{2} \theta_{12}=0,40_{-0,07}^{+0,10}[98]$.

\footnotetext{
${ }^{6}$ Neutrino estéril é aquele que não interage pelas interações fracas de forma usual.
} 
Setor com grande escala de massa: $\Delta m_{31}^{2} \sim 10^{-3} \mathrm{eV}^{2}$

A explicação mais simples e direta dos dados de neutrinos atmosféricos é a de oscilação $\nu_{\mu} \rightarrow \nu_{\tau}$ no vácuo. Outros canais de oscilação estão praticamente descartados.

De fato, o canal $\nu_{\mu} \rightarrow \nu_{e}$ tem implicações diretas nas medidas de neutrinos de reatores. Em particular, assumindo a invariância de CPT, o experimento de CHOOZ deveria ter visto desaparecimento no canal $\bar{\nu}_{e} \rightarrow \bar{\nu}_{\mu}$, o que não ocorreu. Além disso, os fluxos medidos de neutrinos eletrônicos é consistente com as previsões de MC. No caso de oscilação com neutrino estéril, $\nu_{\mu} \rightarrow \nu_{s}$, deveríamos esperar uma queda no sinal de correntes neutras em SK que não foi observada [167].

Uma análise detalhada utilizando as incertezas e os experimentos de aceleradores pode ser encontrada em [68]. Na Figura 4.4 mostramos os resultados atuais na análise de oscilação de neutrinos em duas gerações.

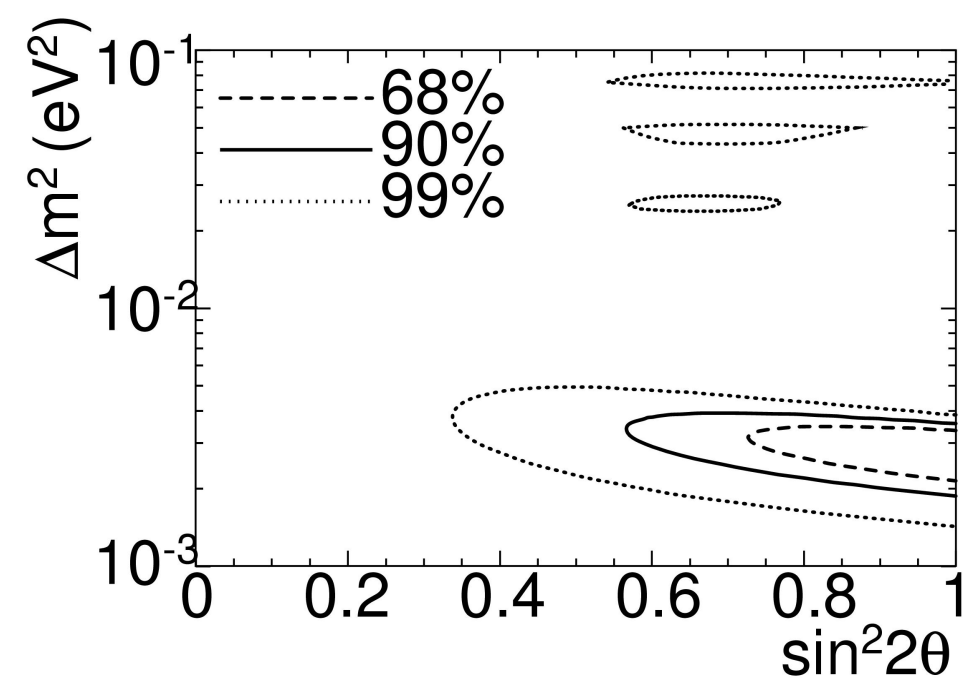

Figura 4.4: Regiões permitidas dos parâmetros de oscilação. Linhas tracejada, sólida e pontilhada representam respectivamente os contornos com $64,4 \%, 90 \%$ e $99 \%$ de confiança. O melhor ajuste é obtido com $\left|\Delta m_{31}^{2}\right|=2,6 \pm 0,2 \times 10^{-3} \quad \mathrm{eV}^{2}$ e $\sin ^{2} 2 \theta_{23}=1,0$ [152].

Além disso, o canal $\nu_{\mu} \rightarrow \nu_{\tau}$ não inclui efeitos de matéria, já que não existem $\mu$ ou $\tau$ na trajetória dos neutrinos. Assim, como o sinal das diferenças quadradas de massa não pode ser investigado para oscilações apenas no vácuo, demonstrado pela Eq. 4.6, $\Delta m_{31}^{2}$ é apenas obtido em módulo.

Resultado de LSND e MiniBooNE: $\Delta \mu^{2} \sim 1 \mathrm{eV}^{2}$

O experimento de LSND [147] apresentou indício de oscilação numa terceira escala de massa quadrada, $\sim 1 \mathrm{eV}^{2}$, o que não é consistente com a hipótese de oscilação de neutrinos em três famílias. 
Para que possamos observar uma terceira escala de massa quadrada nas diferenças de massa é necessário a inclusão de uma quarta massa, ou seja, um quarto autoestado de massa. Desta necessidade surgiu a idéia de neutrino estéril, afinal, como comentado no primeiro capítulo o MP só permite a existência de três neutrinos ativos leves.

Os resultados acima analisados normalmente não consideram o resultado de LSND pelo fato de este ser o único sem confirmação de outro experimento independente. Antes de MiniBooNE, KARMEN já havia descartado uma região permitida por LSND. Os dados divulgados em abril de 2007 por MiniBooNE [149], eliminam com mais de $3 \sigma$ a hipótese de que LSND pode ser explicada por oscilação no contexto de duas gerações. As primeiras análises com os dados desses três experimentos são apresentadas na Figura 4.5. Recentemente no cenário chamado de $3+2$ com dois neutrinos estéreis os resultados de MiniBooNE foram estudados e estão em perfeito acordo com a evidência de aparecimento de LSND graças a possibilidade de violação CP disponível em tais esquemas de oscilação [168].
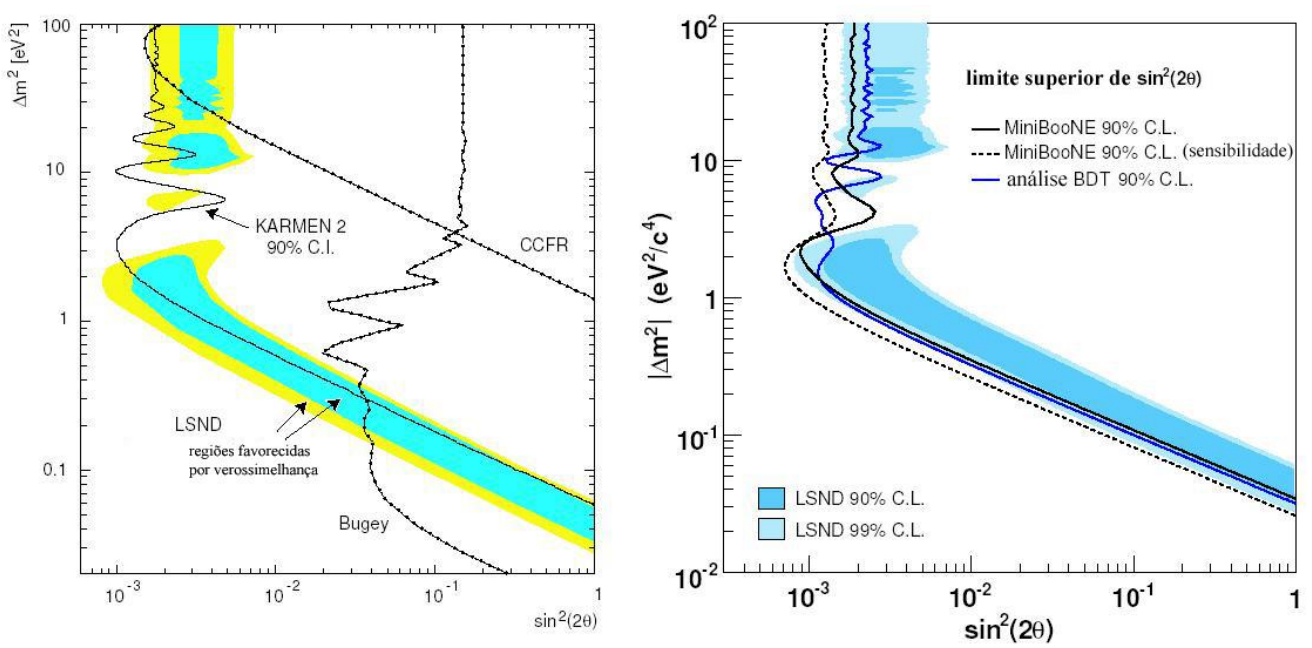

Figura 4.5: Na esquerda estão as regiões permitidas para oscilações $\nu_{e} \rightarrow \nu_{\mu}$ do experimento de LSND comparada com as regiões excluídas por KARMEN, CCFR e Bugey. Na direita, a região excluída por MiniBooNE [68, 147, 149].

\section{Ângulo pequeno: $\theta_{13} \sim 0$}

O problema das análises feitas em duas gerações é que, na redução de três famílias para duas, perdemos informações no terceiro ângulo de mistura $\theta_{13}$ e na fase $\delta$ de violação CP. Esta última sempre vem acompanhada do ângulo pequeno, o que dificulta obter informações sobre seu valor.

Um método que podemos utilizar é através dos experimentos de antineutrinos 
de reatores. A probabilidade de sobrevivência destes tem a forma:

$$
P_{e e}^{\text {reator }} \simeq 1-\sin ^{2} 2 \theta_{13} \sin ^{2}\left(\frac{\Delta m_{31}^{2} x}{4 E}\right)
$$

onde a partir da solução exata no vácuo, devemos utilizar as aproximações $\left|\Delta m_{31}^{2}\right| \simeq\left|\Delta m_{32}^{2}\right|$ e $\Delta m_{21}^{2} \ll E / x$, que são facilmente asseguradas para $\Delta m_{21}^{2} \lesssim$ $10^{-4} \mathrm{eV}^{2}$ e os valores típicos de experimentos de reatores LBL.

Como pode ser observado na Figura 4.6 que apresenta o resultado de antineutrinos de reatores, o experimento de CHOOZ é o mais restritivo na ordem de $\Delta m_{31}^{2} \sim 10^{-3} \mathrm{eV}^{2}$, com $\sin ^{2} 2 \theta \lesssim 0,1$.

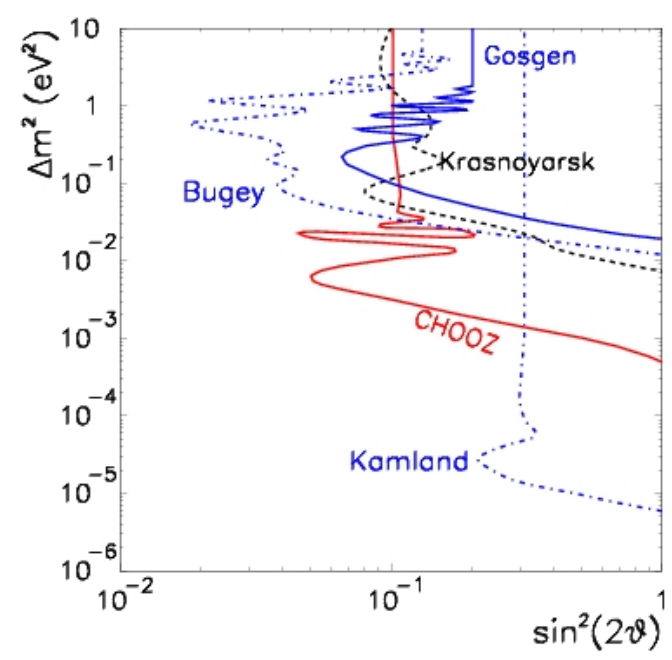

Figura 4.6: Os resultados de antineutrinos de reatores colocam um limite superior no valor do ângulo $\theta_{13} \simeq 0$, mas não o conseguem determinar precisamente. 


\subsection{Resumo da análise dos resultados}

A Figura 4.7 junta os resultados mais importantes num mesmo gráfico.

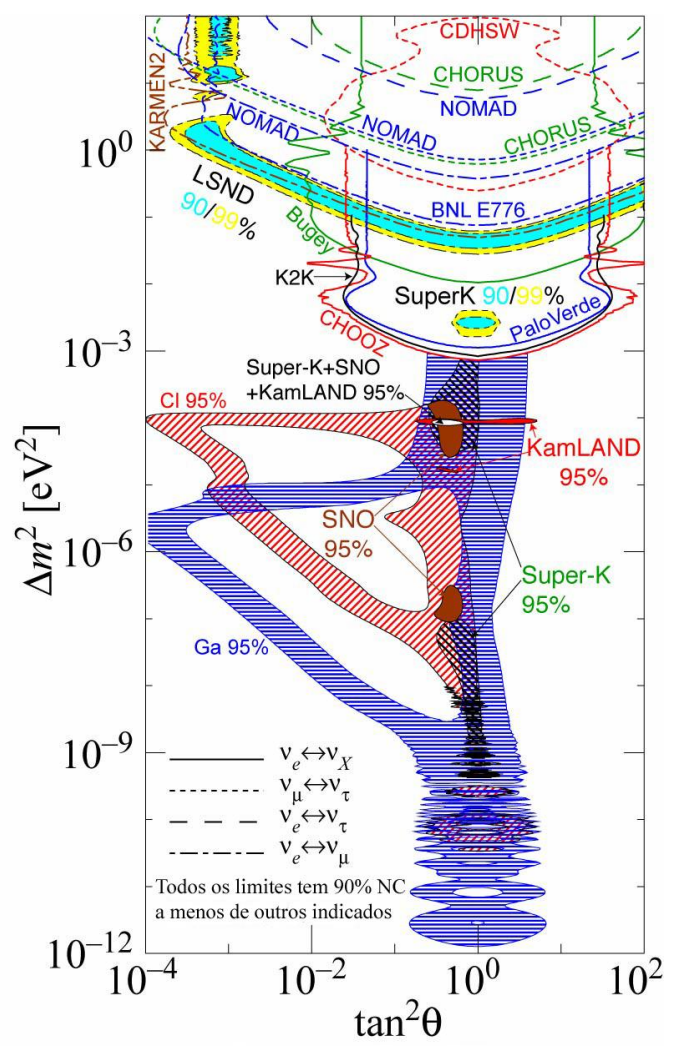

Figura 4.7: Regiões favorecidas ou excluídas das diferenças de massas ao quadrado e ângulos de mistura. As referências dos dados utilizados podem ser encontrados em http://hitoshi.berkeley.edu/neutrino/ref2006.html.

A Tabela 4.3 mostra os valores ajustados dos seis parâmetros de oscilação.

\begin{tabular}{|c|c|}
\hline Parâmetro & Valor $1 \sigma(3 \sigma)$ \\
\hline \hline$\Delta m_{21}^{2}$ & $7,9_{-0,28}^{+0,27}\left(_{-0,89}^{+1,1}\right) \times 10^{-5} \mathrm{eV}^{2}$ \\
\hline$\left|\Delta m_{31}^{2}\right|$ & $2,6 \pm 0,2(0,6) \times 10^{-3} \mathrm{eV}^{2}$ \\
\hline$\theta_{12}$ & $33,7 \pm 1,3\left(_{-3,5}^{+4,3}\right)^{\circ}$ \\
\hline$\theta_{23}$ & $43,3_{-3,8}^{+4,3}\left({ }_{-8,8}^{+9,8}\right)^{\circ}$ \\
\hline$\theta_{13}$ & $0_{-0,0}^{+5,2}\left(_{-0,0}^{+11,5}\right)^{\circ}$ \\
\hline$\delta$ & $\in\left[0^{\circ}, 360^{\circ}\right]$ \\
\hline
\end{tabular}

Tabela 4.3: Valores com melhor ajuste dos seis parâmetros de oscilação de neutrinos em 3 gerações [68].

Finalmente, escrevemos a matriz de mistura com 90\% de confiança [68]:

$$
U=\left(\begin{array}{lll}
0,81 \rightarrow 0,85 & 0,53 \rightarrow 0,58 & 0,00 \rightarrow 0,12 \\
0,32 \rightarrow 0,49 & 0,52 \rightarrow 0,69 & 0,60 \rightarrow 0,76 \\
0,27 \rightarrow 0,46 & 0,47 \rightarrow 0,64 & 0,65 \rightarrow 0,80
\end{array}\right)
$$




\section{Capítulo 5}

\section{Mecanismos de geração de massa}

Neste capítulo fazemos uma breve apresentação dos mecanismos que geram massa para neutrinos em modelos com simetria $S U(2)_{L} \times U(1)_{Y}$. A necessidade da inclusão de massas decorre das evidências apresentadas nos capítulos anteriores para a oscilação de sabores no setor de neutrinos. Não existe nenhuma simetria que impeça massas não nulas para neutrinos, o que sugere que a oscilação seja induzida por massa.

A discussão dos diferentes mecanismos de geração de massa é importante pois traz informação sobre o estudo das oscilações de neutrinos.

$\mathrm{Na}$ primeira parte discutimos um método de análise de diagramas de interação útil para obtenção de parâmetros quando a massa para os neutrinos é gerada por correções radiativas. Na segunda parte comentamos a nulidade das massas dos neutrinos no atual MP, o que faz com que qualquer termo de massa de neutrinos esteja além do MP. Nas seguintes seções estão apresentados os principais mecanismos geradores de massa para neutrinos dentro de um modelo com simetria similar ao do MP: $S U(2)_{L} \times U(1)_{Y}$. Não é feita análise com modelos unificadores ou supersimétricos, pela não necessidade no que segue. Na última seção demonstramos a equivalência dos modelos para o estudo das probabilidades de oscilação.

\subsection{O método dos diagramas de sabor}

É conveniente utilizar os diagramas de Feynman em cálculos para obter amplitudes de transição utilizadas para o cálculo de quantidades físicas, como seções de choque. Entretanto, questões de massa e quebra de simetria ficam mais claras quando trabalhadas em diagramas na chamada base de sabor.

Nos diagramas de Feynman, linhas externas correspondem à partículas físicas. Essas partículas decorrem da lagrangeana final da teoria. No método dos 

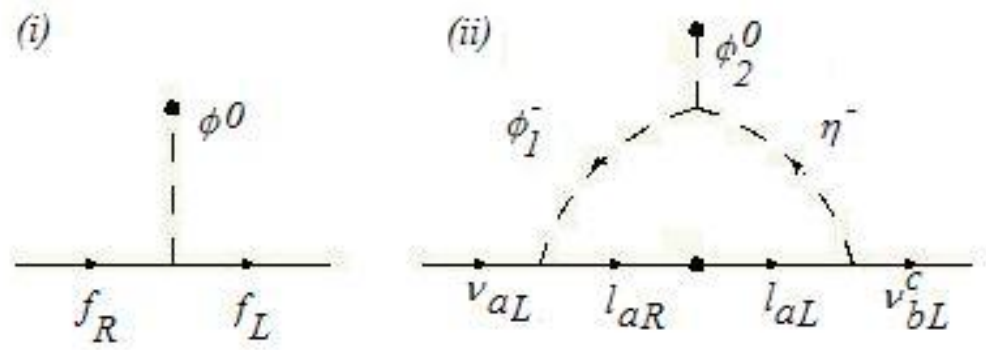

Figura 5.1: Em (i) mostramos o diagrama que representa a contribuição do vev para a geração de massa dos férmions. Em (ii) o diagrama na base de sabor de ordem mais baixa que gera massa para os neutrinos no modelo de Zee.

diagramas de sabor, desenhamos diagramas de Feynman utilizando os campos que aparecem na lagrangeana invariante de calibre. No caso de férmions, isso significa que tratamos os campos quirais de mão esquerda e direita separadamente. Para o campo de Higgs usual do MP, usamos o campo clássico original que aparece antes da quebra de simetria, e não o campo quântico obtido pela flutuação deste campo pelo seu valor esperado de vácuo (vev). Como férmions carregados não possuem massa antes da quebra de simetria, a geração de massa após a quebra de simetria é representada pela Fig. 5.1 (i). O ponto negro no final da linha de $\phi^{0}$ representa sua aniquilação no vácuo, que manifesta o fato de $\phi^{0}$ possuir vev não nulo. Isso representa também que a massa dos férmions pode ser encarada como uma constante de acoplamento entre campos de quiralidades opostas.

Para finalizar essa introdução ao método dos diagramas de sabor observamos o exemplo da Fig. 5.1 (ii). Este diagrama é um exemplo de geração de massa para neutrinos que será discutido em 5.4. O ponto negro na linha interna fermiônica é uma abreviação para o diagrama da Fig. 5.1 (i). Neste caso, um breve olhar no diagrama nos diria que a contribuição para a amplitude correspondente ao laço deveria apresentar:

- um fator de acoplamento $f_{a b}$ correspondente ao vértice com $\eta^{-}$;

- um fator $m_{l}$ da massa inserida;

- um fator $v_{2}$ do vev de $\phi_{2}^{0}$;

- um fator do acoplamento com $\phi^{-}$e $l$ que é $m_{l} / v$.

Este tipo de diagrama também é útil para observar diretamente a quebra de números quânticos globais, no caso é fácil ver que $B-L$ é violado. 


\subsection{O Modelo Padrão implica massa nula para neutrinos}

O MP [19] é baseado na simetria:

$$
G_{M P}=S U(3)_{C} \times S U(2)_{L} \times U(1)_{Y}
$$

O vev do dubleto de Higgs $\phi \sim\left(0, \frac{1}{2},-1\right)$ quebra a simetria para $G_{M P} \rightarrow$ $S U(3)_{C} \times U(1)_{E M}$. As componentes indicam respectivamente o isospin forte, fraco e a hipercarga do multipleto.

A massa dos férmions tem origem nas interações de Yukawa, no setor de léptons

$$
-\mathcal{L}_{\text {Yukawa }}=g^{l} \overline{D_{L}} \phi l_{R}+\text { h.c. },
$$

após a quebra espontânea de simetria, onde $D_{L}=\left(\begin{array}{ll}\nu_{L} & l_{L}\end{array}\right)^{T}$. Essas interações implicam em neutrinos sem massa. Poderíamos pensar que neutrinos obteriam massa através de correções radiativas se estas correções induzissem ao aparecimento de termos efetivos do tipo $g_{i j}^{\nu} \phi \phi D_{L, i} D_{L, j}$. Entretanto tais termos não podem aparecer, como pode ser entendido analisando as simetrias acidentais do MP:

$$
G_{M P}^{\text {global }}=U(1)_{B} \times U(1)_{e} \times U(1)_{\mu} \times U(1)_{\tau}
$$

Termos gerando massa para neutrinos por correções radiativas apresentam $B-$ $L= \pm 2$, enquanto que o número leptônico no MP, sendo uma simetria global, não é violado em nenhuma ordem de perturbação [166].

Somente efeitos não perturbativos poderiam dar origem à massa de neutrinos no MP. De fato, para garantir a renormalizabilidade de uma teoria, a condição de cancelamento de anomalias só é válida para simetrias de calibre. Entretanto, a contribuição da anomalia para a não conservação da corrente de número bariônico tem o mesmo valor que do número leptônico, e sendo assim efeitos não perturbativos no setor de calibre não podem induzir violação da simetria $B-L$, ou seja, $U(1)_{B-L}$ é um subgrupo não anômalo das simetrias globais [166].

Portanto se quisermos neutrinos massivos sem quebrar $B-L$ devemos incluir novos campos no MP - os neutrinos de mão direita. De outra forma, $B-L$ deverá ser violada por algum termo novo. Existem duas formas de violar $B-L$ :

1. Incluindo termos que violam explicitamente a simetria $B-L$. Neste caso podemos pensar em expandir o setor fermiônico incluindo novos férminos como neutrinos de mão direita. Desta forma seremos levados ao mecanismo de gangorra [169]. Se não, podemos expandir o setor escalar, introduzindo 
novos bósons de Higgs. Assim, dependendo do modelo escolhido os neutrinos recebem massa à nível de árvore [170] ou por correções radiativas de primeira [171] ou segunda ordem [172]. Além destes, podemos estudar expansões em ambos os setores $[173,174]$.

2. Incluindo termos que violarão $B-L$ espontaneamente. Aparecem partículas nestes modelos que recebem o nome de Majorons e dão espaço à novos canais de decaimento, cujas não observações em laboratórios colocam limites nos acoplamentos da teoria. Modelos desse tipo não serão abordados aqui $[22]$.

\subsection{Expansão no setor fermiônico}

Se neutrinos têm massa, devemos incluir termos de massa para eles no MP.

Neste ponto nos perguntamos quais termos de massa devem ser incluídos. De um lado podemos pensar que neutrinos são partículas fermiônicas como as demais e assim introduzir os seus respectivos campos de mão direita no MP e atribuir-lhes massas de Dirac. As massas no MP são geradas pelos acoplamentos de Yukawa quando o bóson de Higgs $\phi$ quebra a simetria do modelo adquirindo vev. Aqui não há violação de $B-L$.

Por outro lado, como só observamos neutrinos de mão esquerda e antineutrinos de mão direita, podemos pensar que neutrinos são as suas próprias antipartículas e desta forma partículas de Majorana (veja Apêndice B), com termos de massa violando $B-L$ por duas unidades. Neste último caso não precisamos necessariamente incluir novos férmions ao MP.

Se incluirmos $\nu_{R}$ também de Majorana, e atribuirmos à ele grande valor de massa para explicar a sua não observação em laboratórios, chegaremos ao mecanismo de gangorra que explica a pequenez das massas dos neutrinos de mão esquerda observados.

\subsubsection{Termo de massa de Dirac}

Incluindo três neutrinos de mão direita, eles recebem massa de maneira análoga aos quarks,

$$
-\mathcal{L}_{Y u k a w a}=g_{a b}^{\nu} \overline{D_{a, L}} \tilde{\phi} \nu_{b, R}+\text { h.c. } \rightarrow m_{D}^{a b} \overline{\nu_{a, L}} \nu_{b, R}+\text { h.c. }=-\mathcal{L}_{D},
$$

onde $\tilde{\phi}=i \sigma_{2} \phi^{*}$, os índices $a, b$ se referem as gerações e a flecha indica a quebra de simetria. 


\subsubsection{Termos de massa de Majorana}

Se neutrinos são partículas que possuem termos de massa apenas do tipo de Majorana, não precisamos incluir nenhum mecanismo de geração de massa, já que ambos os campos $\nu_{L}$ e seu conjugado se transformam de acordo com $S U(2)$. No Apêndice B é explicado que ao introduzirmos neutrinos de mão direita, obtemos os seguintes termos de massa:

$$
-\mathcal{L}_{L}^{M}=\frac{1}{2} m_{L} \overline{\nu_{L}^{c}} \nu_{L}+\text { h.c. e }-\mathcal{L}_{R}^{M}=\frac{1}{2} m_{R} \overline{\nu_{R}^{c}} \nu_{R}+\text { h.c. }
$$

onde $\nu_{L}$ e $\nu_{R}$ representam os campos de $\nu=\left(\begin{array}{lll}\nu_{e} & \nu_{\mu} & \nu_{\tau}\end{array}\right)^{T}$ de mão esquerda e direita respectivamente.

\subsubsection{Termos de massa Dirac-Majorana e mecanismo de gangorra}

O caso mais geral é obtido combinando os três termos de massa. Escrevendo $\mathcal{L}_{D+M}=\mathcal{L}_{D}+\mathcal{L}_{L}^{M}+\mathcal{L}_{R}^{M}$ e usando o resultado $\overline{\nu_{L}} \nu_{R}=\overline{\nu_{L}^{c}} \nu_{R}^{c}$, obtemos [175]

$$
-\mathcal{L}_{D+M}=\frac{1}{2}\left(\begin{array}{cc}
\overline{\nu_{L}} & \overline{\nu_{R}^{c}}
\end{array}\right)\left(\begin{array}{cc}
m_{L} & m_{D} \\
m_{D}^{T} & m_{R}
\end{array}\right)\left(\begin{array}{c}
\nu_{L}^{c} \\
\nu_{R}
\end{array}\right)+\text { h.c. }
$$

Devemos digonalizar essa matriz de massa para encontrar as partículas físicas [22, 176]. Neste caso, para simplificar a análise, estudamos o caso de apenas uma geração. Além disso, como discutido, supomos $m_{R} \gg m_{D}$ e $m_{L}=0$ [169]. Nestas condições, uma simples conta fornece os autovalores

$$
m_{1} \simeq m_{R} \quad \text { e } \quad m_{2} \simeq m_{D}^{2} / m_{R}
$$

O mais massivo coincidiria com o auto-estado quase-de-mão-direita e o menos massivo, com o de quase-de-mão-esquerda. Como $m_{D}$ vem dos acoplamentos de Yukawa, é natural supor que seja da mesma ordem das massas dos outros férmions da mesma geração. Dado que apenas os neutrinos mais leves são observados, $m_{R}$ estaria numa escala muito acima da interação fraca, $10^{2} \mathrm{GeV}$, e seria de ordem $m_{R} \gtrsim 10^{9} \mathrm{GeV}$ para acomodar as massa dos neutrinos com $m_{2} \lesssim 1 \mathrm{eV}$ e $m_{D} \lesssim 1 \mathrm{GeV}$, que é aproximadamente a massa do tau (lépton mais pesado).

Além das massas, ao analisarmos os auto-estados para $m_{1}$ e $m_{2}$ denotados por $n_{1}$ e $n_{2}$ respectivamente, obtemos $n_{1}=n_{1}^{c}$ e $n_{2}=-n_{2}^{c}$. Desta forma, ambos neutrinos são partículas de Majorana. Em geral, o mesmo resultado é obtido para mais gerações. 


\subsection{Expansão no setor escalar}

O MP é um modelo mínimo, ou seja, só inclui termos necessários para explicar os resultados observados. Assim, no setor escalar só é incluido um bóson, conhecido como bóson escalar de Higgs, necessário para quebrar a simetria $S U(2)_{L} \times U(1)_{Y}$ e também gerar massa das outras partículas. Entretanto, nada impede que se inclua mais termos neste setor, já que alguns deles não alteram o sucesso do MP - os singletos de $S U(2)$.

No MP existem somente dois tipos de multipletos de léptons

$$
D_{L} \sim\left(\frac{1}{2},-1\right) \quad \text { e } \quad l_{R} \sim(0,-2)
$$

Aqui a primeira componente indica o isospin fraco do multipleto e a segunda, a hipercarga $Y$. Formando todas as combinações bilineares possíveis desses léptons obtemos quais os tipos de bósons de calibre que podem se acoplar aos férmions e ainda manter a simetria do MP:

$$
\begin{array}{cccc}
D_{L} \otimes l_{R} & \frac{1}{2} \otimes 0=\frac{1}{2} & Y=-1 & \phi \text { dubleto de Higgs } \\
D_{L} \otimes D_{L} & \frac{1}{2} \otimes \frac{1}{2}=0 \oplus 1 & Y=-2 & \eta^{+} \text {singleto, } \Delta \text { tripleto } \\
l_{R} \otimes l_{R} & 0 \otimes 0=0 & Y=-4 & k^{++} \text {singleto }
\end{array}
$$

Os singletos possuem a vantagem de não interagir com os bósons do MP, mantendo assim o sucesso dessa teoria.

Os neutrinos são considerados partículas de Majorana pois não há adição de férmions nestes modelos. Portanto a simetria $B-L$ deve ser violada para aparecer os termos de massa. A idéia é tentar incluir apenas um bóson no MP, para mantê-lo mínimo, entretanto somos conduzidos, em alguns casos, à introdução de mais de um.

\subsubsection{Modelo com um tripleto}

Um tripleto, $\vec{\Phi}$, nada mais é do que uma partícula com isospin fraco 1. Assim ele possui três componentes e deve se transformar por $S U(2)$ na sua representação adjunta. Além disso, ele interage com os bósons de interação da força fraca $Z^{0}$ e $W^{ \pm}$.

É fácil ver que $\overline{D_{L}^{c}} \vec{\sigma} D_{L}$ também se tranforma de acordo com a representação adjunta de $S U(2)$. Portanto, o objeto $\overline{D_{L}^{c}} \vec{\sigma} \cdot \vec{\Phi} D_{L}$ comporta-se como um singleto. Costuma-se escrever o tripleto em sua forma matricial $2 \times 2, \Delta=\vec{\Phi} \cdot \vec{\sigma}$. Sob uma transformação $S \in S U(2)$ tem-se $D_{L} \rightarrow S D_{L}$ e $\Delta \rightarrow S \Delta S^{\dagger}$. Nestes termos, pela conservação da carga elétrica, vemos que 


$$
\Delta=\vec{\Phi} \cdot \vec{\sigma}=\left(\begin{array}{cc}
H^{+} & \sqrt{2} H^{++} \\
\sqrt{2} H^{0} & -H^{+}
\end{array}\right)
$$

$\operatorname{com} \vec{\Phi}^{T}=\left(\begin{array}{lll}\Phi_{1} & \Phi_{2} & \Phi_{3}\end{array}\right)=\left(\begin{array}{cc}\frac{1}{\sqrt{2}}\left(H^{0}+H^{++}\right), & \frac{-i}{\sqrt{2}}\left(H^{0}-H^{++}\right), \quad H^{+}\end{array}\right)$.

Os termos a serem incluídos no MP neste modelo são

$$
-\mathcal{L}=f_{a b} \overline{D_{a, L}^{c}} \Delta D_{b, L}+t \phi^{\dagger} \Delta \tilde{\phi}+\text { h.c. }+\frac{1}{2} M^{2} \operatorname{Tr} \Delta^{\dagger} \Delta,
$$

além dos termos de massa e interação para o tripleto, provenientes de suas interações com $\phi$ e sua derivada covariante, lembrando que $\phi$ é o bóson de Higgs usual do MP. As massas dos neutrinos serão geradas se o tripleto apresentar um vev não nulo, isto é $\left\langle H^{0}\right\rangle_{0}=v_{T} / \sqrt{2}$.

Primeiramente, a matriz dos termos de acoplamento para o tripleto é simétrica, ou seja, $f_{a b}=f_{b a}$. Isto ocorre pois a lagrangeana sendo uma função bilinear de férmions deve satisfazer a estatística de Fermi. Explicitando os fatores, observamos que $i \sigma_{2} \vec{\sigma}$ (um tensor simétrico) contrai os índices do espaço $S U(2)$, enquanto $C$ que é antisimétrico contrai os índices espinoriais. Portanto, a matriz dos acoplamentos do espaço de sabores deve ser simétrica. Em seguida, vemos que a lagrangeana deve violar $B-L$. De fato, mesmo que designemos um número leptônico duplo ao tripleto para manter a conservação deste número no primeiro termo, o termo- $t$ a violará explicitamente.

Analisando os resultados obtidos das derivadas covariantes e dos vevs não nulos, obtemos a seguinte correção ao MP para as massas dos bósons [170]

$$
\begin{aligned}
& M_{W}^{2}=\frac{1}{4} g^{2} v^{2} \rightarrow \frac{1}{4} g^{2}\left(v^{2}+2 v_{T}^{2}\right) \\
& M_{Z}^{2}=\frac{1}{4}\left(g^{2}+g^{\prime 2}\right) v^{2} \rightarrow \frac{1}{4}\left(g^{2}+g^{\prime 2}\right)\left(v^{2}+4 v_{T}^{2}\right),
\end{aligned}
$$

onde usando os dados do LEP precisamos ter $\left|v_{T} / v\right| \lesssim 0.03$ [177], onde $v \sim 10^{2}$ $\mathrm{GeV}$. Contudo, se as constantes de acoplamento forem da ordem de 0.01 ou 0.1 , para explicar o porquê as massas dos neutrinos são ao menos seis ordens de grandeza menor que a dos léptons carregados da mesma geração este vev deverá ser bem menor, mais precisamente $v_{T} \sim 0.1 \div 1 \mathrm{eV}$.

Transformando todos os campos por $U(1)$, o vev do dubleto de Higgs $\phi, v$, sempre pode ser escolhido real e positivo. Entretanto, por causa do termo-t no potencial, não encontramos nenhuma simetria para $v_{T}$ ser escolhido real, e o denotamos por $v_{T}=w e^{i \gamma}$.

Uma hipótese plausível de análise é supor $M \sim v$ e $|t| \ll v[178]$. Neste caso, minimizando o potencial devido as interações entre $\Delta$ e $\phi$ obtém-se $w \sim|t|$, 
como desejado. Além disso, sejam os campos reais $\varphi_{R}, \varphi_{I}, H_{R}$ e $H_{I}$ definidos por $\phi^{0}=\frac{1}{2}\left(v+\varphi_{R}+i \varphi_{I}\right)$ e $H^{0}=\frac{1}{\sqrt{2}} e^{i \gamma}\left(w+H_{R}+i H_{I}\right)$. Então os autovalores de massa correspondentes são

$$
\begin{aligned}
& m_{R 1}^{2} \simeq c_{1} v^{2}+\frac{\left(c_{2}-2 q\right)^{2}}{c_{1}-q} w^{2} \\
& m_{R 2}^{2} \simeq q v^{2}-\left[\frac{\left(c_{2}-2 q\right)^{2}}{c_{1}-q}-c_{3}\right] w^{2} \\
& m_{I 1}^{2}=q\left(v^{2}+4 w^{2}\right) \\
& m_{I 2}^{2}=0
\end{aligned}
$$

em que $c_{1}, c_{2}, c_{3}$ são constantes supostamente de ordem 1 , provenientes do potencial minimizado, e $q=|t| / w$. As massas dos campos- $R$ são dadas até ordem $w^{2}$.

$\mathrm{O}$ autovalor nulo corresponde à combinação linear $2 w H_{I}+v \varphi_{I}$, que é proporcional ao pseudo bóson de Goldstone associado com $Z^{0}$. No primeiro modelo com um tripleto proposto por Gelmini e Roncadelli [170], o termo- $t$ não havia sido incluído, $q=0$. Sendo assim, o bóson de Goldstone proveniente da quebra espontânea de $U(1)_{L}$, chamado de Majoron, não adquiria massa, pois esta é proporcional à $q$. Além disso, neste caso $m_{R 2}^{2}$ é da ordem de $w^{2}$, e portanto, o $Z^{0}$ pode decair no Majoron e escalar leve com uma largura de decaimento de dois sabores de neutrinos [179]. Então este modelo de Gelmini e Roncadelli está descartado pelos resultados do LEP.

A inclusão do termo-t possibilitou manter consistente com resultados experimentais o modelo do tripleto, pois desta forma vê-se que as partículas resultantes podem ter massa bem superiores às produzidas hoje em laboratório. Entretanto, outra hipótese pode ser feita com relação às ordens de grandeza dos acoplamentos para obter $v_{T} \lesssim 1 \mathrm{eV}$, esta é $M,|t| \gg v[180]$. Assim, após outro cálculo extenso $\left|v_{T}\right| \simeq|t| v^{2} / M^{2}$.

De certo modo, o mecanismo para obter massas pequenas para neutrinos através da adição de um tripleto é análogo ao mecanismo de gangorra, já que em ambos os casos temos que introduzir uma segunda escala muito maior (ou menor) que a escala da teoria eletrofraca.

Ainda no modelo com tripleto, vários outros casos já foram estudados na literatura, por exemplo a adição de um quarto neutrino estéril ao MP tentando explicar os resultados obtidos no experimento LSND [178] assim como a adição de dois tripletos ao invés de um só [180]. 


\subsubsection{Modelo de Zee}

O chamado modelo de Zee [171] se diferencia dos tradicionais por adicionar massa aos neutrinos através de correções radiativas. Pela adição de escalares que não interagem com a força fraca, o método é mais fácil de se implementar por não ter restrições provenientes dos vínculos do MP.

No Apêndice B é comentado que propagadores de Majorana são iguais aos de Dirac. Desta forma, após um processo de renormalização obtemos

$$
S_{F}(\not p)=\frac{i}{\not p-m_{n}-\Sigma(\not p)}
$$

onde $m_{n}$ é a massa não renormalizada do neutrino e $\Sigma(\not p)$ é sua auto-energia. Além disso, o contratermo de massa é definido por $\delta m=m_{n}-m$, em que $m$ é a massa física. Esta massa física é definida pelo pólo do propagador, ou seja, $\left[\not p-m_{n}-\Sigma(\not p)\right]_{p=m}=0$. Como a correção da massa não renomalizada à massa física é proveniente de loops, temos $m=m_{n}+O(f)$, em que $f$ é a constante de acoplamento. A primeira correção é da ordem deste acoplamento, e levando apenas esta ordem em consideração, obtemos:

$$
\delta m=\Sigma_{1}(\not p=m) \simeq \Sigma_{1}\left(m_{n}=0\right),
$$

onde $\Sigma_{1}(\not p) \sim O\left(f^{2}\right)$. Em geral não precisamos nos preocupar com a convergência dos loops pois a renormalização da teoria está garantida pelo teorema de 't Hooft.

Em seu primeiro modelo [171], Zee tentou incluir o singleto com carga simples, $\eta^{+}$. Entretanto, como este não pode desenvolver vev não nulo, $B-L$ se mantém conservado caso designemos um valor duplo de número leptônico para $\eta^{+}$. Não há termos de interação desta partícula com o dubleto de Higgs que violem tal simetria, pois $\bar{\phi} \tilde{\phi} \eta^{+}=\epsilon_{i j} \phi_{i} \phi_{j} \eta^{+}$se anula pela antisimetria de $\epsilon$. A saída proposta por Zee foi considerar dois dubletos de Higgs, $\phi_{1,2}$. Desta forma, o termo adicionado ao MP é

$$
-\mathcal{L}=f_{a b} \overline{D_{L}^{c}} D_{L} \eta^{+}+t \phi_{1}^{\dagger} \tilde{\phi}_{2} \eta^{+}+\text {h.c. }
$$

Pela mesma análise feita no Modelo do tripleto, $f_{a b}=-f_{b a}$.

O primeiro caso considerado foi o mais simples, quando apenas um dos dubletos se acopla aos léptons [182]. Sendo assim, a geração de massa para neutrinos seria dada pelo diagrama da Fig. 5.1 (ii). Pelo método dos diagramas de sabor, obtemos facilmente que $m_{a b}=A f_{a b}\left(m_{a}^{2}-m_{b}^{2}\right)$, onde A é uma constante de proporcionalidade. Diferente dos modelos anteriores, este oferece uma previsão 
para a mistura e massa dos neutrinos. Normalmente modelos que geram massa através de correções radiativas fornecem padrões para essas matrizes.

Definindo $\tan \alpha=\frac{f_{\mu \tau}}{f_{e \tau}}\left(1-\frac{m_{\mu}^{2}}{m_{\tau}^{2}}\right)$ e $\sigma=\frac{f_{e \mu}}{f_{e \tau}} \frac{m_{\mu}^{2}}{m_{\tau}^{2}} \cos \alpha$, e desprezando a massa do elétron frente às massas do muon e do tau, a matriz de massa dos neutrinos pode ser reescrita como

$$
M=m_{0}\left(\begin{array}{ccc}
0 & \sigma & \cos \alpha \\
\sigma & 0 & \sin \alpha \\
\cos \alpha & \sin \alpha & 0
\end{array}\right) \text {, }
$$

onde $m_{0}=A m_{\tau}^{2} f_{\tau e} / \cos \alpha$. Assim, se um dos parâmetros $\sigma, \cos \alpha$ ou $\sin \alpha$ for pequeno, os autovalores de massa correspondentes a essa matriz considerando até primeira ordem serão

$$
\begin{aligned}
& m_{1}=-m_{\star} K_{1}^{2}(1-\sigma \sin \alpha \cos \alpha), \\
& m_{2}=m_{\star} K_{2}^{2}(1+\sigma \sin \alpha \cos \alpha), \\
& m_{3}=-2 m_{\star} K_{3}^{2} \sigma \sin \alpha \cos \alpha,
\end{aligned}
$$

em que $K_{i}^{2}= \pm 1$, para que os autovalores de massa sejam positivos. A constante $m_{\star}$ apresenta uma relação simples com $m_{0}$ : se o parâmetro pequeno for $\sigma$, então $m_{\star}=m_{0}$; senão, $m_{\star}=m_{0} \sqrt{1+\sigma^{2}}$ na primeira ordem de teoria de perturbação em termos do menor parâmetro. É natural pensar que o menor parâmetro seja $\sigma$, por envolver a razão $m_{\mu}^{2} / m_{\tau}^{2}$. Neste caso, a matriz de mistura que diagonaliza $M$ deve ser

$$
O=\frac{1}{\sqrt{2}}\left(\begin{array}{ccc}
\sqrt{2} \sin \alpha & \cos \alpha & \cos \alpha \\
-\sqrt{2} \cos \alpha & \sin \alpha & \sin \alpha \\
0 & -1 & 1
\end{array}\right)
$$

que é inconsistente com os dados experimentais, pois para ter um ângulo quase máximo no setor atmosférico precisamos de $\sin \alpha \simeq 1$ e assim $\cos \alpha \simeq 0$, levando à um ângulo máximo também no setor solar, o que já foi descartado pelos experimentos de SNO e KamLAND [86, 97]. Um resultado parecido é obtido se considerarmos $\sin \alpha \ll 1$ [183].

Na tentativa de salvar o modelo, devemos generalizá-lo para o caso onde os dois dubletos se acoplam aos léptons. Isto é feito para um padrão simples de massas e mistura de neutrinos em [184], aliviando assim algumas restrições e mantendo o modelo sustentável. Entretanto, o limite $\left|f_{a b}\right| \lesssim 10^{-4}$ deve ser respeitado. 


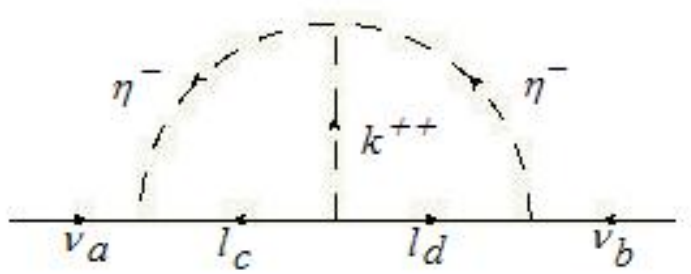

Figura 5.2: Diagrama na base de sabor de menor ordem para geração de massa para os neutrinos no Modelo de Zee-Babu.

\subsubsection{Modelo de Zee-Babu}

O último modelo a se considerar é aquele onde o singleto de carga dupla, $k^{++}$, é adicionado ao MP. Assim como no modelo anterior, a simples inclusão desta partícula não proporciona nenhum termo de interação que viole $B-L$. Contudo, como apontado por Babu [172], a situação muda ao incluirmos juntamente o singleto com carga única, $\eta^{+}$. Desta forma, o termo adicionado ao MP é

$$
-\mathcal{L}=F_{a b} \bar{l}_{a, R}^{c} l_{b, R} k^{++}+f_{a b} \overline{D_{a, L}^{c}} D_{b, L} \eta^{+}+t \eta^{-} \eta^{-} k^{++}+h . c .
$$

Um ponto interessante neste modelo é o fato de adicionarmos quase todos os escalares possíveis ao MP, com excessão somente do tripleto. Além disso, o diagrama de menor ordem que pode gerar massa para os neutrinos é de ordem 2 e está apresentado na Fig. 5.2, isto oferece uma explicação para a pequenez das massas dos neutrinos. A matriz de massa correspondente a este gráfico é

$$
m_{a b}=8 t m_{c} m_{d} f_{a c} F_{c d} f_{b d} I_{c d}
$$

onde

$$
I_{c d}=\int \frac{d^{4} p}{(2 \pi)^{4}} \int \frac{d^{4} q}{(2 \pi)^{4}} \frac{1}{p^{2}-m_{c}^{2}} \frac{1}{q^{2}-m_{d}^{2}} \frac{1}{p^{2}-m_{\eta}^{2}} \frac{1}{q^{2}-m_{\eta}^{2}} \frac{1}{(p-q)^{2}-m_{k}^{2}} .
$$

Outro resultado interessante é que por $f_{a b}$ ser antisimétrica, o determinante da matriz $m_{a b}$ se anula quando há três gerações. Então, algum dos autovalores é zero. Isto não implica que haja um neutrino sem massa, pois esta pode ser proveniente de correções de ordens ainda mais altas. Assim, é de se esperar um autovalor consideravelmente menor do que os outros dois, podendo dar origem à uma hierarquia de massas consistente com os experimentos de oscilação.

Com a finalidade de se obter fórmulas analíticas simples e assim analisar o modelo, podemos desprezar as massas dos léptons no denominador, já que a massa dos escalares devem ser significativamente maiores [185]. Então $I_{c d} \simeq$ $I=\frac{1}{\left(16 \pi^{2}\right)^{2}} \frac{1}{m_{\eta}^{2}} \tilde{I}\left(\frac{m_{k}^{2}}{m_{\eta}^{2}}\right)$, para uma quantidade sem dimensão $\tilde{I}$ definida por $\tilde{I}(r)=$ 
$-\int_{0}^{1} d x \int_{0}^{1-x} d y \frac{1-y}{x+(r-1) y+y^{2}} \log \frac{y(1-y)}{x+r y}$.

Vários resultados de decaimentos e reações podem ser usados para se chegar nas seguintes restrições: $m_{\eta}>590 \mathrm{GeV}(5.04 \mathrm{TeV})$ no caso de hierarquia normal (invertida) [186], $\left|F_{\mu \mu}\right|:\left|F_{\mu \tau}\right|:\left|F_{\tau \tau}\right| \simeq 1:\left(m_{\mu} / m_{\tau}\right):\left(m_{\mu} / m_{\tau}\right)^{2}$ e $\left|f_{a b}\right|,\left|F_{a b}\right| \lesssim$ 0.1 [185]. Finalmente, este modelo poderá ser confirmado pelo LHC, através da

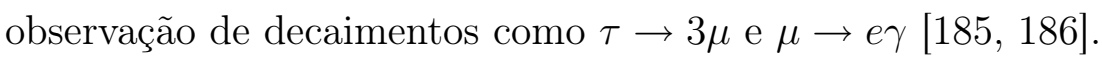

\subsection{Expansão nos dois setores}

Além dos modelos mencionados, poderíamos pensar em combiná-los. Afinal não necessariamente apenas o setor escalar ou fermiônico deve ser extendido.

Um exemplo seria adicionar neutrinos de mão direita e obter massas de Dirac provenientes de acoplamentos com o dubleto de Higgs $\phi$, enquanto os termos de massa de Majorana viriam dos acoplamentos com o tripleto. O resultado final seria novamente o mecanismo de gangorra. A mesma idéia vale para qualquer um dos outros modelos [173].

Além disso, novos modelos podem ser estudados, como a inclusão de dois dubletos e apenas um neutrino de mão direita. Neste caso, apenas uma das três massas dos neutrinos viria à nivel de árvore, a segunda viria por correção radiativa de ordem mais baixa. Novamente o mecanismo de gangorra estaria presente [174].

\subsection{Massas e oscilação}

Foi visto nas seções anteriores os principais mecanismos conhecidos para geração de massa no setor de neutrinos dentro de um modelo que mantenha a simetria $S U(2)_{L} \times U(1)_{Y}$. Contudo ainda não fizemos a ligação entre as massas e a oscilação. Esta última parte do capítulo se dedica à demonstrar que a oscilação induzida por massa é independente do mecanismo de geração adotado.

No caso da expansão no setor escalar, ambas a geração através de correções radiativas e por novos escalares com vev não nulo conduzem à inclusão de um termo efetivo de massa de Majorana. Desta forma, a análise se reduz à mesma feita para a expansão no setor fermiônico.

Na expansão do setor fermiônico, por simplicidade, começaremos a análise para os neutrinos de Dirac. Neste caso a lagrangeana que descreve as massas dos léptons e as interações de CC são dadas por:

$$
-\mathcal{L}_{C C}^{D}=\frac{g}{\sqrt{2}} \overline{E_{a, L}} \gamma^{\mu} \nu_{a, L} W_{\mu}^{-}+m_{l}^{a b} \overline{E_{a, L}} E_{b, R}+m_{D}^{a b} \overline{\nu_{a, L}} \nu_{b, R}+\text { h.c. }
$$


onde as primeiras letras do alfabeto indicam os sabores: $a, b=e, \mu, \tau$ e os léptons carregados estão representados pelo vetor $E=\left(\begin{array}{ccc}e^{-} & \mu^{-} & \tau^{-}\end{array}\right)^{T}$.

O teorema da decomposição singular nos diz que as matrizes de massa podem ser diagonalizadas, com valores na diagonal não negativos, por duas matrizes unitárias. Definindo novos estados leptônicos por estas matrizes:

$$
E_{i, L}=V_{L}^{i a} E_{a, L}, \quad E_{j, R}=V_{R}^{j b} E_{b, R}, \quad \nu_{i, L}=U_{L}^{i a} \nu_{a, L}, \quad \nu_{j, R}=U_{R}^{j b} \nu_{b, R},
$$

onde $i, j=1,2,3$ e já que $V_{L}^{\dagger} m_{l} V_{R}=M_{l}$ e $U_{L}^{\dagger} m_{D} U_{R}=M_{\nu}$, então a Eq. (5.22) pode ser reescrita nesta base, chamada de base de autoestados de massa, resultando em

$$
-\mathcal{L}_{C C}^{D}=\frac{g}{\sqrt{2}} \overline{E_{i, L}} \gamma^{\mu}\left(V_{L}^{\dagger} U_{L}\right)_{i j} \nu_{j, L} W_{\mu}^{-}+M_{l}^{i} \overline{E_{i, L}} E_{i, R}+M_{\nu}^{j} \overline{\nu_{j, L}} \nu_{j, L}+\text { h.c. } .
$$

Isto mostra claramente que a matriz que mistura as interações e que vai dar origem a oscilação de neutrinos entre os estados de sabor e de massa é $U=V_{L}^{\dagger} U_{L}$, portanto unitária.

Além disso, podemos mudar a fase dos campos leptônicos desde que a fase introduzida aos campos de mão esquerda anulem às dos campos de mão direita nos termos de massa. Desta forma, estas fases só irão aparecer no termo de CC, podendo assim ser absorvida na matriz de mistura. Se as novas fases são dadas pelas matrizes diagonais $P_{l}$ para os léptons carregados e $P_{\nu}$ para os neutrinos, então a matriz de mistura se transforma por $U \rightarrow P_{l}^{\dagger} U P_{\nu}$. No caso de neutrinos de Majorana não há como redefinir as fases dos neutrinos sem tornar o termo de massa complexo, pois $\overline{\nu^{c}} \nu=\nu^{T} C \nu$ (veja Apêndice B). Isto implica que neste caso $P_{\nu}$ deve ser a identidade.

A diferença então entre os casos de Dirac e Majorana se resume a quantidade de fases que aparecem na matriz de mistura. Mais explicitamente $U_{M a j}=U_{D i r} P_{\nu}$. Entretanto, como veremos no próximo capítulo, a equação de evolução dos neutrinos é tal que as hamiltonianas são $H_{M a j}=P_{\nu}^{\dagger} H_{D i r} P_{\nu}$ o que acarreta em probabilidades de transição iguais.

Conclui-se portanto que os efeitos de oscilação são de modo geral insensíveis aos tipos de termos de massa dos neutrinos. Se por um lado isto simplifica o estudo de oscilação, por outro lado isto significa que as medidas dos parâmetros de oscilação não nos fornece informação sobre a origem das massas. Já era esperado que a oscilação não auxiliasse na distinção entre neutrinos de Dirac e Majorana, pois a simetria $U(1)_{B-L}$ não é violada pelo fenômeno. 


\section{Capítulo 6}

\section{Equação de evolução na matéria}

Num estudo analítico detalhado das probabilidades de oscilação de neutrinos na matéria não podemos aceitar a abordagem convencional de oscilação proposta no capítulo anterior. É necessário investigar os motivos e sob quais condições as expressões utilizadas nas análises dos dados são válidas.

Neste capítulo propomos um procedimento de demonstração de cada resultado utilizado, que trazem à tona as condições de sua validade. Em primeiro lugar, apresentamos uma demonstração do potencial de matéria e sob quais hipóteses ele é válido. Após isso, é obtida a equação de Schrödinger de evolução a partir da lagrangeana do MP, como desejado. Uma extensa apresentação da parametrização da matriz de mistura é feita, pois seus vários parâmetros indicam a presença de fenômenos físicos. Na última seção são mostrados alguns resultados importantes e gerais das probabilidades de oscilação que são soluções da equação de evolução.

\subsection{Efeito de matéria}

O objetivo desta seção é demonstrar o seguinte resultado:

No limite de baixas energias comparadas à massa de $W, m_{W} \sim 80 \mathrm{GeV}$, e dentro de um meio estático, isotrópico e homogêneo, tal que os únicos léptons presentes são os elétrons, a evolução dos neutrinos é dada sob o efeito de um potencial da forma $V(x)=\sqrt{2} G_{F} N_{e}(x)$, com $G_{F}$ a constante de interação fraca e $N_{e}(x)$ a densidade de elétrons no meio.

De fato a lagrangeana que descreve a evolução dos neutrinos no MP é a soma de uma parte cinética que envolve a massa (independente do mecanismo de geração) com uma parte de interação. Essa última provém da simetria $S U(2)_{L} \times$ 

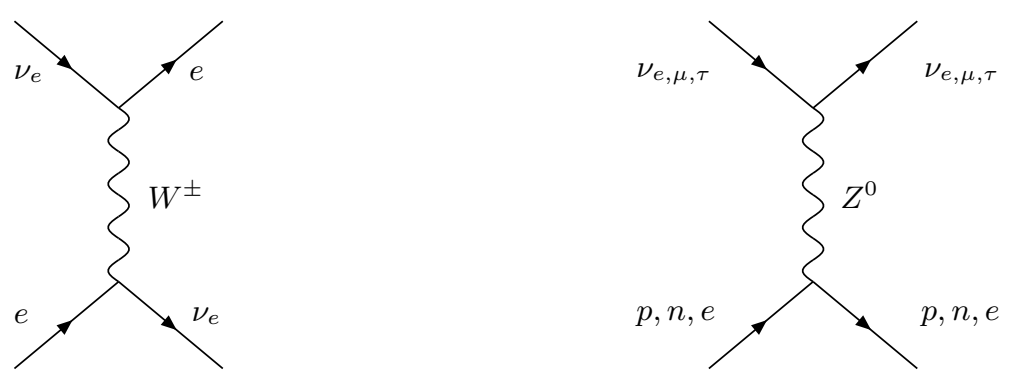

Figura 6.1: Diagramas de espalhamento dos neutrinos na matéria com troca de um bóson.

$U(1)_{Y}$. Entretanto, a baixas energias comparadas às massas dos bósons vetoriais $W^{ \pm}$e $Z^{0}$, os acoplamentos dos bósons fracos têm seus maiores efeitos através de processos que envolvem uma troca de bóson fraco virtual.

Pode-se mostrar que [187]

$$
\begin{aligned}
\left\langle W_{\mu}^{+}(p) W_{\nu}^{-}(-p)\right\rangle & =\frac{g_{\mu \nu}}{p^{2}-m_{W}^{2}} \\
\left\langle Z_{\mu}^{0}(p) Z_{\nu}^{0}(-p)\right\rangle & =\frac{g_{\mu \nu}}{p^{2}-m_{Z}^{2}}
\end{aligned}
$$

onde \langle\rangle indica a média sobre todas as polarizações. Estes resultados estão corretos até diagramas que trocam $W$ ou $Z$ da ordem de $m_{f} / m_{W}$, onde $m_{f}$ indica a massa do férmion envolvido. Quando podemos desprezar o momento $p$ comparado à $m_{W}<m_{Z}$, ou seja, com $p^{2} \ll m_{W}^{2} \simeq(80 \mathrm{GeV})^{2}$, a teoria eletrofraca pode ser tratada pela teoria efetiva de 4 vértices. Desta forma a lagrangeana de interação dos neutrinos pode ser descrita por

$$
\mathcal{L}_{i n t}=\mathcal{L}_{W}+\mathcal{L}_{Z}=-\frac{g^{2}}{m_{W}^{2}} J_{W}^{\mu-} J_{\mu W}^{+}-\frac{g^{2}}{m_{Z}^{2}} J_{Z}^{\mu} J_{\mu Z}
$$

onde $\frac{g^{2}}{8 m_{W}^{2}}=\frac{G_{F}}{\sqrt{2}}$, e as correntes são $J_{W}^{\mu-}=\frac{1}{\sqrt{2}} \bar{\nu}_{L} \gamma^{\mu} l_{L}, J_{\mu W}^{+}=\frac{1}{\sqrt{2}} \bar{l}_{L} \gamma_{\mu} \nu_{L}$ e $J_{Z}^{\mu}=$ $\frac{1}{2 \cos \theta_{W}} \bar{\nu}_{L} \gamma^{\mu} \nu_{L}$.

\subsubsection{Interações de correntes carregadas}

Analisamos primeiro as interações de CC. Utilizando a transformação de Fierz, basta considerar $\mathcal{L}_{W}=-\frac{G_{F}}{\sqrt{2}} j^{\mu} \bar{\nu} \gamma_{\mu}\left(1-\gamma_{5}\right) \nu$, definindo $j^{\mu}=\bar{l} \gamma^{\mu}\left(1-\gamma_{5}\right) l$. A priori não há motivos para haver evolução da matéria, portanto esta é suposta estática. Assim seu efeito sobre o estado do neutrino pode ser estudado em média: $j^{\mu} \rightarrow\left\langle j^{\mu}\right\rangle=\left\langle\bar{l} \gamma^{\mu}\left(1-\gamma_{5}\right) l\right\rangle$, com \langle\rangle indicando a média sobre o meio. Na matéria ordinária não encontra-se nem $\mu^{ \pm}$e nem $\tau^{ \pm}$, o que leva à corrente não nula somente para os elétrons. Como só são considerados léptons de mão esquerda na corrente, tem-se a igualdade $\left\langle\bar{l} \gamma^{\mu}\left(1-\gamma_{5}\right) l\right\rangle=2\left\langle j_{L}^{\mu}\right\rangle$. Chamando de $N_{e}(x)$ a densidade de elétrons do meio, e $\vec{v}_{e}$ a velocidade dos mesmos, obtém-se 
as relações:

- $\left\langle j_{L}^{0}\right\rangle=\frac{1}{2}\left\langle N_{e}\right\rangle$, para um meio homogêneo (sem polarizações);

- $\left\langle\vec{j}_{L}\right\rangle=\left\langle\vec{v}_{e}\right\rangle=0$, para um meio isotrópico;

concluindo que $\left\langle j^{\mu}\right\rangle=\delta^{\mu 0} N_{e}(x)$. Portanto $\mathcal{L}_{W}=-\sqrt{2} G_{F} N_{e} \bar{\nu}_{L} \gamma_{0} B \nu_{L}$, onde $B$ é uma matriz que age no espaço de sabores com $B=\operatorname{diag}(1,0,0)$. Podemos interpretar este termo dependente da densidade como uma contribuição à energia potencial

$$
V_{C C}(x)=\sqrt{2} G_{F} N_{e}(x) B
$$

\subsubsection{Interações de correntes neutras}

Olhando agora para as contribuições da corrente neutra, encontraremos de maneira análoga as seguintes contribuições para os potenciais efetivos de todos os sabores de neutrinos:

$$
V_{N C}(x)=\sqrt{2} G_{F} \sum_{f} N_{f}\left[I_{f}-2 \sin ^{2} \theta_{W} Q_{f}\right],
$$

onde $f$ são os férmions encontrados na matéria: elétrons, prótons e nêutrons, $Q_{f}$ é a carga de $f$ e $I_{f}$ é a componente de isospin de $f$, tomando próton e nêutron como integrantes de um dubleto de núcleon. Então, para o próton $Q=1 \mathrm{e}$ $I=\frac{1}{2}$, enquanto para o elétron $Q=-1$ e $I=-\frac{1}{2}$. Além disso, a matéria usual é neutra, $N_{e}=N_{p}$, assim as contribuições do elétron e do próton se cancelam e o potencial efetivo só possui contribuição do nêutron, que é

$$
V_{N C}(x)=-\sqrt{2} G_{F} N_{n}(x) / 2 .
$$

Como a influência de $\mathcal{L}_{Z}$ será idêntica na matéria para as três gerações de neutrinos, acarretará em um termo na equação de evolução proporcional à identidade. Desta forma, o mesmo não implica mudança nos resultados das probabilidades de oscilação de onde encontramos o resultado desejado.

\subsection{Equação efetiva de Schrödinger}

Uma partícula de spin 1/2 como é o caso do neutrino, é governada pela equação de Dirac. Entretanto, no estudo das probabilidades de oscilação de sabor de neutrinos é sempre utilizada na literatura uma equação de Schrödinger para descrever a evolução destes. Seguindo [188], esta seção demonstrará o seguinte resultado: 
A evolução de neutrinos monocromáticos de mão esquerda, com massas de Dirac ou Majorana, no limite de altas energias $m_{\nu} / E \ll 1$, sob o efeito de um potencial devido à presença de matéria satisfazendo as condições acima se reduz da equação de Dirac

$$
(i \not \partial-M) \nu=V \gamma^{0} \frac{1-\gamma_{5}}{2} B \nu,
$$

que está escrita na base de autoestados de sabor, com $M$ uma matriz de massa não necessariamente diagonal e $B=\operatorname{diag}(1,0,0)$, para uma equação de Schrödinger

$$
i \frac{d}{d x} \nu=\left[\frac{1}{2 E} U \operatorname{diag}\left(m_{1}^{2}, m_{2}^{2}, m_{3}^{2}\right) U^{\dagger}+V(x) \operatorname{diag}(1,0,0)\right] \nu
$$

se considerarmos apenas termos até ordem $M^{2} / E^{2}$ e $V / E$, com o potencial satisfazendo a seguinte regularidade $\frac{\partial_{x} V}{V} \ll 1 / \lambda_{\nu}$, em que $\lambda_{\nu}$ é o comprimento de onda do neutrino para o problema. Para antineutrinos, considerados como as soluções de energia negativa, a equação continua valendo com $V \leftrightarrow-V$ e $U \rightarrow U^{*}$.

De fato, utilizando o resultado da seção anterior, a lagrangeana completa do problema é dada pelo potencial mais a parte cinética:

$$
\mathcal{L}_{\nu}=\frac{i}{2} \bar{\nu} \partial_{\mu} \gamma^{\mu} \nu-\bar{\nu} M \nu-\frac{G_{F}}{\sqrt{2}} \delta_{\mu 0} N_{e} \bar{\nu} \gamma^{\mu}\left(1-\gamma_{5}\right) B \nu
$$

lembrando que a matriz de massa $M$ não é necessariamente diagonal. As equações de Euler-Lagrange neste caso são

$$
(i \not \partial-M) \nu=\frac{G_{F}}{\sqrt{2}} \delta_{\mu 0} N_{e} \gamma^{\mu}\left(1-\gamma_{5}\right) N \nu
$$

porém é mais interessante trabalhar com as projeções quirais. Aplicando $P_{L} \mathrm{e}$ $P_{R}$ respectivamente na equação acima, e usando $P_{L} P_{R}=0$ o par de equações obtido para ambos neutrinos de Dirac ou Majorana é (veja Apêndice B):

$$
\begin{aligned}
i \not \partial \nu_{R}-M \nu_{L} & =0 \\
i \not \partial \nu_{L}-M \nu_{R} & =\sqrt{2} G_{F} N_{e} \gamma^{0} B \nu_{L}
\end{aligned}
$$

O $\nu_{R}$ da segunda equação é eliminado fazendo uso da primeira. A partir daí, procura-se soluções estacionárias (autofunções da energia). Como é possível controlar a energia de um neutrino autoestado de interação, coloca-se $\nu_{\alpha}(x, t)=$ $\nu_{\alpha}(x) e^{-i E t}, \operatorname{com} \alpha=e, \mu, \tau$. Obtém-se

$$
\left(E^{2}-M^{2}+\triangle\right) \nu_{L}=\sqrt{2} G_{F} i \gamma^{\mu} \partial_{\mu}\left(N_{e} \gamma^{0} B \nu_{L}\right)
$$

Para neutrinos relativísticos $E \simeq p \Rightarrow i \gamma^{\mu} \partial_{\mu} \nu \simeq 0$, assim $\partial_{0} \nu \simeq \gamma^{i} \gamma^{0} \partial_{i} \nu, \operatorname{com} i=$ 
1,2,3. Supondo $\frac{\partial_{i} N_{e}}{N_{e} E} \ll 1$ (equivalente à $\frac{\partial_{x} V}{V} \ll 1 / \lambda_{\nu}$, onde $\lambda_{\nu}$ é o comprimento de onda do neutrino para o problema e $V=\sqrt{2} G_{F} N_{e}$ ), chega-se à

$$
\left(E^{2}-M^{2}+\triangle\right) \nu_{L}=2 \sqrt{2} G_{F} N_{e} E B \nu_{L}
$$

Definindo $F=E^{2}-M^{2}-2 \sqrt{2} G_{F} N_{e} E B$ e considerando apenas termos até ordem $M^{2} / E^{2}$ e $V / E$ encontra-se $\sqrt{F}=E-\frac{M^{2}}{2 E}-\sqrt{2} G_{F} N_{e} B$, mesmo que $M$ e $B$ não comutem. A equação de segunda ordem $(F+\triangle) \nu_{L}=0$ se divide em duas de primeira, que passando para o problema unidimensional (propagação do neutrino em apenas uma direção) se torna

$$
\left(\sqrt{F}-i \frac{d}{d x}\right)\left(\sqrt{F}+i \frac{d}{d x}\right) \nu_{L}(x)=0
$$

As duas soluções desta equação caminham em sentidos opostos. Omitindo a solução refletida no espalhamento, subtraindo o termo proporcional a identidade e diagonalizando $M^{2}=U \operatorname{diag}\left(m_{1}^{2}, m_{2}^{2}, m_{3}^{2}\right) U^{\dagger}$, com a matriz de mistura $U$ definida na última seção do capítulo anterior, chega-se à equação desejada.

Para encontrar a equação de propagação para antineutrinos, além de modificar as equações de Euler-Lagrange adequadamente deve-se tomar as soluções estacionárias de energia negativa. A mudança que aparece nas equações de EulerLagrange induzem a substituição $U \rightarrow U^{*}$. Enquanto que a escolha da solução estacionária de energia negativa faz aparecer um sinal negativo na parte direita da Eq. (6.11). Este sinal é associado à $N_{e}$, e portanto para passar da equação de neutrinos para antineutrinos basta fazer a mudança $V \rightarrow-V$.

Outras demonstrações para a Eq. (6.7) podem ser encontradas nas Refs. $[189,190]$.

\subsection{Parametrização da matriz de mistura}

Para finalizar a forma da equação de evolução de neutrinos monocromáticos para o estudo de oscilações devemos parametrizar a matriz de mistura $U$. Como foi discutido $U \in U(3)$, o grupo das matrizes unitárias em 3 dimensões. Além disso, o número de fases em $U$ é diferente nos casos de neutrinos de Dirac e Majorana, mas pela Eq. (6.7) as fases adicionais não modificam as fórmulas de probabilidades de transição.

A diferença entre uma matriz $B \in S U(n)$ e uma matriz $U \in U(n)$ é apenas uma fase, ou seja,

$$
U=B \times \operatorname{diag}\left(1, \ldots, e^{i \phi}\right) .
$$

Para simplificar o estudo da parametrização olhemos para as matrizes de $S U(n)$. 
Uma matriz genérica $B \in S U(n)$ pode ser parametrizada por uma sequência de vetores complexos unitários $\xi, \ldots, \beta, \alpha$ de dimensões $n, n-1, \ldots, 3,2$. Isto é garantido por um teorema [191] que pode ser visto como segue: seja $\Sigma_{n}=$ $\left\{\psi \in \mathbb{C}^{n} \mid \psi^{\dagger} \psi=1\right\}$ o conjunto dos vetores unitários num espaço de Hilbert de dimensão $n$, ou seja, um conjunto real de dimensão $2 n-1$. Como a condição de unitariedade impõe restrição apenas aos módulos das componentes do vetor então o grau de liberdade é de $n$ fases (parâmetros imaginários) e $n-1$ ângulos (parâmetros reais). Qualquer $\psi \in \Sigma_{n}$ pode ser mapeado no vetor $e_{n}=\left(\begin{array}{llll}0 & 0 & \ldots & 0\end{array}\right)$ por um determinado elemento de $S U(n)$. O subgrupo que deixa $e_{n}$ invariante é $S U(n-1)$ nas primeiras $(n-1)$ dimensões e então

$$
\Sigma_{n} \simeq \text { coset space } S U(n) / S U(n-1)
$$

Desta forma, qualquer elemento de $S U(n)$ pode ser unicamente especificado por um par consistindo de um elemento de $S U(n-1)$ e um vetor $\psi \in \Sigma_{n}$. Recursivamente vemos que um elemento $B \in S U(n)$ pode ser parametrizado por $B=B(\xi, \ldots, \beta, \alpha)$, uma sequência de vetores unitários complexos de dimensões $n, n-1, \ldots, 3,2$.

\subsubsection{Número de graus de liberdade de $U(n)$ e $S U(n)$}

Podemos agora contar o número de graus de liberdade da matriz de mistura $U$ no caso genérico de $n$ gerações. Para tanto basta contar para uma matriz de $S U(n)$ e adicionar uma fase.

Do teorema acima, o número de ângulos de $U$ é $N_{A}(n)=1+2+\ldots+(n-1)=$ $\frac{n(n-1)}{2}$, e o número de fases $N_{F}(n)=2+3+\ldots+(n-1)+n+1=\frac{n(n+1)}{2}$.

Portanto o número de graus de liberdade de uma matriz de $U(n)$ é $n^{2}$ com $\frac{n(n-1)}{2}$ ângulos e $\frac{n(n+1)}{2}$ fases, e de $S U(n)$ é $n^{2}-1$ com o mesmo número de ângulos e uma fase a menos, eliminada pela condição de determinante real e positivo.

\subsubsection{Matrizes de $S U(2)$ e $S U(3)$}

Vamos agora parametrizar as matrizes de $S U(n)$. Por conveniência deixaremos o vetor unitário na última coluna, pois quando uma matriz de $S U(n)$ é multiplicada pela direita por uma matriz de $S U(n-1)$ deixando $e_{n}$ invariante, é a última coluna que permanece inalterada. Evidentemente $S U(1)=\{1\}$. Qualquer matriz $A \in S U(2)$ é dada por

$$
A=A(\alpha)=\left(\begin{array}{cc}
\alpha_{2}^{*} & \alpha_{1} \\
-\alpha_{1}^{*} & \alpha_{2}
\end{array}\right) \quad ; \quad \alpha^{\dagger} \alpha=1
$$


Agora vamos construir a matriz de $B(\beta) \in S U(3)$ dependendo apenas dos parâmetros de $\beta \in \Sigma_{3}$. Na forma mais geral temos

$$
B(\beta)=\left(\begin{array}{ccc}
a_{1} & a_{2} & \beta_{1} \\
b_{1} & b_{2} & \beta_{2} \\
c_{1} & c_{2} & \beta_{3}
\end{array}\right)
$$

entretanto podemos fixar 4 parâmetros dos valores de $a_{1,2}, b_{1,2}$ e $c_{1,2}$ referentes aos graus de liberdade que serão atribuídos às componentes de $\alpha$. Contudo a restrição desses elementos não deve implicar em nenhuma restrição aos valores de $\beta$. Pela troca de linhas e colunas não perdemos a generalidade escolhendo $a_{2}=0$ e $a_{1}>0$ (se $a_{1}>0$ e $a_{2}>0$ então restringiríamos o valor de $\beta_{1}$ ). Pelas condições de unitariedade da matriz, com a escolha de $a_{1}$ real e a fase de $b_{2}$ o oposto da fase de $\beta_{3}$, encontramos para $\left|\beta_{1}\right|<1$, a matriz

$$
B(\beta)=\left(\begin{array}{ccc}
P^{-1} & 0 & \beta_{1} \\
-P \beta_{1}^{*} \beta_{2} & P \beta_{3}^{*} & \beta_{2} \\
-P \beta_{1}^{*} \beta_{3} & -P \beta_{2}^{*} & \beta_{3}
\end{array}\right) \quad, \quad P=\left(1-\left|\beta_{1}\right|^{2}\right)^{-1 / 2}
$$

que está em $S U(3)$, com

$$
B(\beta)=\left(\begin{array}{ccc}
1 & 0 & 0 \\
0 & P \beta_{3}^{*} & P \beta_{2} \\
0 & -P \beta_{2}^{*} & P \beta_{3}
\end{array}\right) \times\left(\begin{array}{ccc}
P^{-1} & 0 & \beta_{1} \\
0 & 1 & 0 \\
-\beta_{1}^{*} & 0 & P^{-1}
\end{array}\right)
$$

assim como sua transposta e permutações de linhas e colunas. A escolha da ordem das linhas e colunas só define a representação do espaço $S U(3) / S U(2)$. Então, qualquer matriz $B \in S U(3),\left|B_{13}\right|<1$, pode ser univocamente escrita como $B=B(\beta) \cdot\left(\begin{array}{cc}A(\alpha) & 0 \\ 0 & 1\end{array}\right)$, obtendo [192]

$$
B(\alpha, \beta)=\left(\begin{array}{ccc}
P^{-1} \alpha_{2}^{*} & P^{-1} \alpha_{1} & \beta_{1} \\
-P \beta_{1}^{*} \beta_{2} \alpha_{2}^{*}-P \beta_{3}^{*} \alpha_{1}^{*} & -P \beta_{3}^{*} \beta_{2} \alpha_{1}+P \beta_{3}^{*} \alpha_{2} & \beta_{2} \\
-P \beta_{1}^{*} \beta_{3} \alpha_{2}^{*}+P \beta_{2}^{*} \alpha_{1}^{*} & -P \beta_{1}^{*} \beta_{3} \alpha_{1}-P \beta_{2}^{*} \alpha_{2} & \beta_{3}
\end{array}\right)
$$

\subsubsection{Fases de Dirac e Majorana em $U(n)$ e $S U(n)$}

A matriz de mistura $U$ pode ter suas fases redefinidas através da absorção de fases pelos campos que a multiplicam na lagrangeana, como foi descrito na última seção do capítulo anterior. A quantidade de fases que podem ser incluídas nesta transformação depende do tipo de massa do neutrino.

Se os neutrinos são de Dirac, ambos os lados da matriz $U \in U(n)$ podem ser 
multiplicados por uma matriz diagonal de fases, ou seja, $2 n$ fases. Entretanto, apenas $2 n-1$ fases são efetivamente utilizadas, pois deve-se descontar uma fase global que pode ser absorvida pela redefinição de todas as outras. Desta forma, o grau de liberdade de $U$ diminui para $\frac{(n-1)(n-2)}{2}$ fases físicas. Na análise de uma matriz de $S U(n)$, as matrizes de fase devem possuir determinante real e positivo, e portanto apenas $2 n-2$ fases são absorvidas, levando assim ao mesmo grau de liberdade de uma matriz unitária.

No caso de neutrinos de Majorana, a análise é parecida. Como apenas o lado em que os léptons carregados se encontram é utilizado para redefinir as fases da matriz de mistura, somente $n$ fases podem ser utilizadas, com isso o grau de liberdade da matriz unitária cai para $\frac{n(n-1)}{2}$ fases físicas. Enquanto se a análise fosse feita para matrizes de $S U(n)$ apenas $n-1$ fases seriam absorvidas, levando ao mesmo resultado.

Portanto, conclui-se que a análise da parametrização pode ser feita para $U \in$ $S U(n)$. Além disso, em duas gerações obtemos um ângulo e nenhuma (uma) fase para neutrinos de Dirac (Majorana) e em três gerações obtemos três ângulos e uma (três) fase(s).

\subsubsection{Redefinição de fases em $S U(2)$ e $S U(3)$}

Estudamos então como uma matriz de $S U(2)$ ou de $S U(3)$ se comporta sob uma transformação de fase para massa do tipo de Dirac já que a oscilação é insensível às fases de Majorana.

No caso de $A \in S U(2)$ temos $A^{\prime}=D\left(\varphi^{\prime}\right) A D(\varphi)$, onde $D(\varphi)=\operatorname{diag}\left(e^{i \varphi}, e^{-i \varphi}\right)$ e $D\left(\varphi^{\prime}\right)$ é definido de maneira análoga. Encontramos assim $A^{\prime}=A\left(\alpha^{\prime}\right)$, onde $\alpha_{1}^{\prime}=\alpha_{1} e^{-i\left(\varphi-\varphi^{\prime}\right)}$ e $\alpha_{2}^{\prime}=\alpha_{2} e^{-i\left(\varphi+\varphi^{\prime}\right)}$, ou seja, todas as fases são absorvidas, o que implica em $U$ real.

No caso de $B \in S U(3)$ temos $B^{\prime}=D\left(\theta^{\prime}\right) B D(\theta)$, onde

$$
D(\theta)=\operatorname{diag}\left(e^{i\left(\theta_{1}+\theta_{2}\right)}, e^{-i\left(\theta_{1}-\theta_{2}\right)}, e^{-2 i \theta_{2}}\right)
$$

com $D\left(\theta^{\prime}\right)$ definida de maneira parecida. Então encontramos que $B^{\prime}=B\left(\alpha^{\prime}, \beta^{\prime}\right)$ onde

$$
\begin{array}{ll}
\alpha_{1}^{\prime}=\alpha_{1} e^{i\left(\theta_{1}^{\prime}+\theta_{2}^{\prime}-\theta_{1}+\theta_{2}\right)} ; & \alpha_{2}^{\prime}=\alpha_{2} e^{-i\left(\theta_{1}^{\prime}+\theta_{2}^{\prime}+\theta_{1}+\theta_{2}\right)} \\
\beta_{1}^{\prime}=\beta_{1} e^{i\left(\theta_{1}^{\prime}+\theta_{2}^{\prime}-2 \theta_{2}\right)} ; \quad \beta_{2}^{\prime}=\beta_{2} e^{i\left(-\theta_{1}^{\prime}+\theta_{2}^{\prime}-2 \theta_{2}\right)} ; \quad \beta_{3}^{\prime}=\beta_{3} e^{-2 i\left(\theta_{2}^{\prime}+\theta_{2}\right)},
\end{array}
$$

ou seja, não é difícil de ver que $\arg \left(\alpha_{1} \alpha_{2}^{*} \beta_{1}^{*} \beta_{2} \beta_{3}\right)$ fica invariante sob a transformação de fase. 


\subsubsection{Parametrização de $U$ em duas gerações}

Pela análise feita acima, no caso de duas gerações $U \in U(2)$ depende apenas de um ângulo. Assim $\alpha$ em (6.16) é real. Por sua unitariedade, $\alpha_{1}$ e $\alpha_{2}$ podem ser escritos como senos e cosenos de um ângulo. A escolha destes e do sinal não altera a parametrização, apenas gira o ângulo. No caso tradicional escolhe-se $\alpha_{1}=\sin \theta$ e $\alpha_{2}=\cos \theta$, levando à matriz de mistura:

$$
U=\left(\begin{array}{cc}
\cos \theta & \sin \theta \\
-\sin \theta & \cos \theta
\end{array}\right)
$$

\subsubsection{Parametrização de $U$ em três gerações}

A parametrização de $U$ é dada pela Eq. (6.20) em três gerações. Pela análise feita em 6.3.4 apenas um dos $\alpha_{1}, \alpha_{2}, \beta_{1}, \beta_{2}, \beta_{3}$ deve ser escolhido complexo e todos os outros reais. Sem levar em consideração a fase, existem 9 maneiras independentes de se parametrizar essa matriz que podem ser obtidas através da permutação de linhas, colunas e por transposição [193].

A maneira mais simples de escolher os ângulos é: $\alpha_{1}=\sin \theta_{12}, \alpha_{2}=\cos \theta_{12}$, $\beta_{1}=\sin \theta_{13} e^{-i \delta}, \beta_{2}=\cos \theta_{13} \sin \theta_{23}$ e $\beta_{3}=\cos \theta_{13} \cos \theta_{23}$. Esta escolha leva a parametrização tradicional de Chau-Keung [194]

$$
U=\left(\begin{array}{ccc}
c_{12} c_{13} & s_{12} c_{13} & s_{13} e^{-i \delta} \\
-s_{12} c_{23}-c_{12} s_{13} s_{23} e^{i \delta} & c_{12} c_{23}-s_{12} s_{13} s_{23} e^{i \delta} & c_{13} s_{23} \\
s_{12} s_{23}-c_{12} s_{13} c_{23} e^{i \delta} & -c_{12} s_{23}-s_{12} s_{13} c_{23} e^{i \delta} & c_{13} c_{23}
\end{array}\right)
$$

Aqui $c_{i j}=\cos \theta_{i j}$ e $s_{i j}=\sin \theta_{i j}$, e $\theta_{i j}$ são entendidos como os ângulos que misturam as famílias $i$ e $j$. De fato, temos nesta parametrização a igualdade $U=O_{23} U_{\delta} O_{13} U_{\delta}^{\dagger} O_{12}$ em que $O_{i j}$ é a matriz ortogonal de rotação no plano $i j$

$$
O_{23}=\left(\begin{array}{ccc}
1 & 0 & 0 \\
0 & c_{23} & s_{23} \\
0 & -s_{23} & c_{23}
\end{array}\right), O_{13}=\left(\begin{array}{ccc}
c_{13} & 0 & s_{13} \\
0 & 1 & 0 \\
-s_{13} & 0 & c_{13}
\end{array}\right), O_{12}=\left(\begin{array}{ccc}
c_{12} & s_{12} & 0 \\
-s_{12} & c_{12} & 0 \\
0 & 0 & 1
\end{array}\right)
$$

e $U_{\delta}=\operatorname{diag}\left(1,1, e^{i \delta}\right)$.

Para se obter uma parametrização parecida, $U=O_{23} U_{\delta} O_{13} O_{12}$, que é muito utilizada na literatura, precisamos lembrar que $U \in U(3)$ e portanto sua fórmula mais geral é dada pela Eq. (6.14). Ao se colocar $\phi=\delta$ e observando que $\left[U_{\delta}, O_{12}\right]=0$, obtemos o resultado desejado. 


\subsection{Propriedades das soluções}

No estudo que faremos das probabilidades de oscilação de neutrinos utilizaremos a seguinte equação de evolução em três gerações:

$$
i \frac{d}{d x}|\nu\rangle=\left[\frac{1}{2 E} U \operatorname{diag}\left(0, \Delta m_{21}^{2}, \Delta m_{31}^{2}\right) U^{\dagger}+V(x) \operatorname{diag}(1,0,0)\right]|\nu\rangle,
$$

onde $|\nu\rangle=\left(\begin{array}{lll}\nu_{e} & \nu_{\mu} & \nu_{\tau}\end{array}\right)^{T}, \Delta m_{i j}^{2}=m_{i}^{2}-m_{j}^{2}$ para $i, j=1,2,3$ e a matriz é parametrizada por $U=O_{23} U_{\delta} O_{13} U_{\delta}^{\dagger} O_{12}$ ou $U=O_{23} U_{\delta} O_{13} O_{12}$.

No caso de apenas duas gerações esta equação se reduz à

$$
i \frac{d}{d x}|\nu\rangle=\frac{\Delta m^{2}}{4 E}\left(\begin{array}{cc}
-\cos 2 \theta+A(x) & \sin 2 \theta \\
\sin 2 \theta & \cos 2 \theta-A(x)
\end{array}\right)|\nu\rangle,
$$

onde $|\nu\rangle=\left(\nu_{X} \nu_{Y}\right)^{T}, \Delta m^{2}=m_{2}^{2}-m_{1}^{2} \mathrm{e}$

$$
A(x)=\frac{2 E V(x)}{\Delta m^{2}} .
$$

\subsubsection{Operador de evolução}

Nos interessamos agora à transformação que leva do estado inicial dos neutrinos, $\left|\nu\left(x_{0}\right)\right\rangle$, à um estado final, $|\nu(x)\rangle$. Esta transformação é chamada de operador de evolução, representada aqui por $S\left(x, x_{0}\right)$, e é definida por

$$
|\nu(x)\rangle=S\left(x, x_{0}\right)\left|\nu\left(x_{0}\right)\right\rangle, \quad S\left(x_{0}, x_{0}\right)=I .
$$

Evidentemente esta operação é unitária e satisfaz a mesma equação de Schrödinger que os neutrinos $|\nu(x)\rangle$ com hamiltoniana $H(x)$ :

$$
i \frac{d}{d x} S\left(x, x_{0}\right)=H(x) S\left(x, x_{0}\right)
$$

Por esta abordagem de solução, as probabilidades de oscilação de neutrinos são dadas por

$$
P_{\alpha \beta}(x)=P\left(\nu_{\alpha} \rightarrow \nu_{\beta} ; x\right)=\left|\left\langle\nu_{\beta} \mid \nu(x)\right\rangle\right|^{2}=\left|\left\langle\nu_{\beta}\left|S\left(x, x_{0}\right)\right| \nu_{\alpha}\right\rangle\right|^{2}=\left|S_{\beta \alpha}\right|^{2} .
$$

Além disso, a solução formal para este operador num ponto $x$ qualquer é

$$
\begin{array}{cc}
S\left(x, x_{0}\right)= & \sum_{n=0}^{\infty}(-i)^{n} \int_{x_{0}}^{x} d x_{1} \int_{x_{0}}^{x_{1}} d x_{2} \ldots \int_{x_{0}}^{x_{n-1}} d x_{n} H\left(x_{1}\right) \ldots H\left(x_{0}\right) \\
= & T \exp \left(-i \int_{x_{0}}^{x} d s H(s)\right)
\end{array}
$$


onde $T$ é o chamado operador de ordenamento temporal [195], cuja solução se reduz à $S\left(x, x_{0}\right)=e^{-i H x}$ quando o potencial é constante.

\subsubsection{Probabilidades independentes}

A unitariedade de $S\left(x, x_{0}\right)$ está ligada com a conservação da probabilidade:

$$
\sum_{\alpha} P_{\alpha \beta}=\sum_{\beta} P_{\alpha \beta}=1
$$

Em duas gerações esta conservação implica que as quatro probabilidades de oscilação podem ser escritas em termos de apenas uma. De fato, $P_{X X}+P_{X Y}=1$ e $P_{X X}+P_{Y X}=1$ implicam em $P_{X Y}=P_{Y X}$, de onde escolhendo $P_{X Y}$ como a fórmula independente temos:

$$
\begin{aligned}
P_{X X} & =1-P_{X Y} \\
P_{Y X} & =P_{X Y} \\
P_{Y Y} & =1-P_{X Y}
\end{aligned} .
$$

Em três gerações a situação é mais complicada. A conservação da probabilidade nos fornece seis equações das quais apenas cinco são linearmente independentes. Sendo assim, cinco das nove fórmulas de probabilidades de oscilação podem ser escritas em termos das outras quatro.

Além destas propriedades lineares, existe uma simetria adicional devida à escolha da parametrização da matriz de mistura dada pela Eq. (6.23). Fazendo uma rotação de $-\theta_{23}$ na hamiltoniana obtemos outra que não depende desse ângulo, pois $\left[O_{23}, V(x) \operatorname{diag}(1,0,0)\right]=0$. É simples de ver que o operador de evolução irá rodar pelo ângulo contrário. Chamamos os operadores após a rotação de $H^{\prime}=O_{23}^{T} H O_{23}$ e $S^{\prime}=O_{23} S O_{23}^{T}$. Podemos usar esse operador de evolução que não depende de $\theta_{23}$ para demonstrar algumas relações úteis entre as fórmulas de probabilidades [196].

Escrevemos a transformação $\theta_{23} \rightarrow \theta_{23}+\pi / 2$ por

$$
\tilde{P}_{\alpha \beta} \equiv P_{\alpha \beta}\left(s_{23}^{2} \leftrightarrow c_{23}^{2}, \sin 2 \theta_{23} \rightarrow-\sin 2 \theta_{23}\right), \alpha, \beta=e, \mu, \tau
$$

A rotação permite portanto ver que:

$$
\begin{aligned}
P_{e \tau} & =\left|S_{\tau e}\right|^{2} \\
& =c_{23}^{2}\left|S_{\tau e}^{\prime}\right|^{2}+s_{23}^{2}\left|S_{\mu e}^{\prime}\right|^{2}-\sin 2 \theta_{23} \operatorname{Re}\left[S_{\mu e}^{\prime} S_{\tau e}^{\prime *}\right]
\end{aligned},
$$


e que

$$
\begin{aligned}
P_{e \mu} & =\left|S_{\mu e}\right|^{2} \\
& =c_{23}^{2}\left|S_{\mu e}^{\prime}\right|^{2}+s_{23}^{2}\left|S_{\tau e}^{\prime}\right|^{2}+\sin 2 \theta_{23} \operatorname{Re}\left[S_{\mu e}^{\prime} S_{\tau e}^{\prime *}\right]
\end{aligned}
$$

então usando a Eq. (6.34) conclui-se que $P_{e \tau}=\tilde{P}_{e \mu}$. Do mesmo modo podemos mostrar as relações $P_{\tau \mu}=\tilde{P}_{\mu \tau}$ e $P_{\tau \tau}=\tilde{P}_{\mu \mu}$, enquanto $P_{e e}$ é independente de $\theta_{23}$. Destas três equações, apenas duas são independentes, pois a outra pode ser escrita utilizando as condições de unitariedade.

Portanto chegamos à importante conclusão de que o número de fórmulas independentes para oscilação de neutrinos é de apenas duas, contanto que a dependência no ângulo $\theta_{23}$ seja conhecida. Sendo assim, a escolha destas probabilidades não é livre: não pode ser $P_{e e}$ que não depende de $\theta_{23}$; nem deve ser um par de probabilidades ligadas por reversão temporal $\left(P_{\alpha \beta}\right.$ e $\left.P_{\beta \alpha}\right)$; e nem ir de uma à outra pela transformação $s_{23}^{2} \leftrightarrow c_{23}^{2}, \sin 2 \theta_{23} \rightarrow-\sin 2 \theta_{23}$.

Uma escolha possível, e que é usualmente feita, é usar a transição entre as famílias vizinhas $P_{e \mu}$ e $P_{\mu \tau}$. Usando as relações acima encontramos

$$
\begin{aligned}
P_{e e} & =1-\left(P_{e \mu}-\tilde{P}_{e \mu}\right) \\
P_{e \tau} & =\tilde{P}_{e \mu} \\
P_{\mu e} & =P_{e \mu}-P_{\mu \tau}+\tilde{P}_{\mu \tau} \\
P_{\mu \mu} & =1-P_{e \mu}-\tilde{P}_{\mu \tau} \\
P_{\tau e} & =\tilde{P}_{e \mu}+P_{\mu \tau}-\tilde{P}_{\mu \tau} \\
P_{\tau \mu} & =\tilde{P}_{\mu \tau} \\
P_{\tau \tau} & =1-\left(\tilde{P}_{e \mu}+P_{\mu \tau}\right)
\end{aligned}
$$

Em conclusão, quando trabalhamos com oscilação de neutrinos em duas gerações só precisamos encontrar a expressão analítica de $P_{X Y}$, enquanto em três gerações, só precisamos procurá-las para:

$$
P\left(\nu_{e} \rightarrow \nu_{\mu} ; x\right) \quad \text { e } \quad P\left(\nu_{\mu} \rightarrow \nu_{\tau} ; x\right)
$$

\subsubsection{Hierarquia de massas}

Experimentalmente são obtidas duas escalas de diferenças de massas quadradas. Pela discussão feita no capítulo anterior, $\Delta m_{21}^{2} \ll\left|\Delta m_{31}^{2}\right| \simeq\left|\Delta m_{32}^{2}\right|$.

A determinação do sinal de $\Delta m_{31}^{2}$ ainda é um problema em aberto. Isto significa que temos $m_{1}<m_{2} \ll m_{3}$ ou $m_{3} \ll m_{1}<m_{2}$, que correspondem ao caso de hierarquia normal e invertida respectivamente.

A oscilação no vácuo não nos fornece informação sobre tal sinal, pois as fórmulas de probabilidades obtidas só dependem do módulo. Entretanto, se durante sua trajetória os neutrinos passarem por uma zona em que o efeito de 
matéria aumenta significativamente a probabilidade de transição (chamada de zona de ressonância), então alguma informação podemos obter. Esta zona de ressonância ocorre se $A(x) \sim 1$, onde $A(x)$ foi definido na Eq. (6.27). Isto ocorre com neutrinos solares. De fato o núcleo solar possui uma densidade $100 \mathrm{~g} / \mathrm{cm}^{3}$ o que equivale à um potencial $V_{C C} \sim 10^{-12} \mathrm{eV}$, o que fornece com $\Delta m_{21}^{2} \sim 10^{-5} \mathrm{eV}^{2}$ o valor $A(x) \sim 1$. É desta forma que inferimos o sinal positivo de $\Delta m_{21}^{2}$, diferença de massa relacionada a transição de neutrinos solares.

A outra escala de massa está envolvida na transição entre $\nu_{\mu} \leftrightarrow \nu_{\tau}$ nos neutrinos atmosféricos, em que o potencial efetivo de matéria não entra na equação de evolução desprezando a contribuição de $\nu_{e}$. Desta forma não obtemos informação conclusiva sobre o sinal de $\Delta m_{31}^{2}$. Além disso seria necessário uma densidade $\sim 10^{4} \mathrm{~g} / \mathrm{cm}^{3}$ para neutrinos com energia perto de $20 \mathrm{MeV}$ para que $A(x) \sim 1$ usando esta escala de diferença de massas quadradas. Este cenário é encontrado em supernovas, o que é uma das razões que torna relevante o estudo desses neutrinos. 


\section{Capítulo 7}

\section{Soluções exatas da equação de evolução}

A primeira abordagem à equação de evolução é a procura por soluções exatas. Idealmente gostaríamos de encontrar uma solução no caso de três gerações para um potencial arbitrário. Entretanto tal solução não existe analiticamente. Sendo assim prosseguimos a busca restringindo as formas possíveis do potencial e o número de gerações.

$\mathrm{Na}$ natureza encontramos apenas potenciais nos seguintes meios: interior do Sol, cuja densidade pode ser aproximada por uma exponencial ou uma tangente hiperbólica; interior da Terra, que pode ser dividido em duas camadas de densidade praticamente constante, o núcleo e o manto; em supernovas, cujas densidades ainda não conhecidas perfeitamente são geralmente descritas por potenciais que caem como $x^{-3}$ ou $x^{-5}$; além, evidentemente, do vácuo. É claro que na procura por soluções exatas podemos ainda considerar o caso linear dada sua importância pela possibilidade de aproximação de diversas funções localmente por uma reta.

Outra simplificação que podemos fazer na equação de evolução é reduzir o número de famílias envolvidas. No Capítulo 4 vimos que os resultados experimentais conduzem à divisão do estudo de oscilação em duas escalas de diferenças de massas quadradas, uma solar $\left(\nu_{e} \rightarrow \nu_{\mu, \tau}\right)$ e outra atmosférica $\left(\nu_{\mu} \leftrightarrow \nu_{\tau}\right)$. No próximo capítulo veremos como tal redução é alcançada quando aproximamos $\frac{\Delta m_{21}^{2}}{\Delta m_{31}^{2}} \rightarrow 0$ ou $\theta_{13} \rightarrow 0$.

Nas últimas décadas várias soluções para a equação de evolução foram obtidas. Em duas gerações conhecemos as soluções para $V(x) \propto x[197,198], 1 / x$ [160], $e^{-x}$ [199, 200, 201, 202], e $1+\tanh x$ [203], além do potencial com densidade constante e vácuo. Em três gerações apenas as soluções para os potenciais $V(x) \propto x[197], e^{-x}[202,204]$ e constante (incluindo vácuo) foram apresentadas 
até agora.

No presente projeto estudamos todas essas soluções. No entanto fomos além disso pois ao estudar as Refs. [197, 204], notamos que a utilização de apenas um formalismo independente do número de gerações é capaz de nos conduzir à todas as soluções conhecidas, possibilitando inclusive obter solução para alguns novos potenciais. Chegamos à duas conclusões importantes: existe a mesma quantidade de potenciais com soluções exatas em duas e em três gerações; e além dos casos conhecidos, existem mais potenciais com soluções exatas ${ }^{1}$.

O capítulo se divide em quatro partes: na primeira apresentamos o formalismo utilizado na obtenção das fórmulas exatas; na segunda aplicamos ao caso de duas gerações e analisamos as soluções comparando-as com as propostas na literatura; na terceira parte extendemos as soluções para o caso de três gerações; e na última parte fazemos um comentário geral sobre os outros métodos propostos para resolução da equação de evolução na matéria com potencial constante.

\subsection{Método de resolução}

Nesta seção apresentamos o formalismo utilizado para obtenção das soluções exatas. Para facilitar a análise generalizamos a equação de evolução para $N$ gerações. No que segue $N=2,3$. Entretanto, matematicamente $N$ pode ser superior à 3, mas não inclui neutrinos estéreis pois isto conduziria a inclusão do potencial $V_{N C}$, que não é considerado aqui. Desta forma $N>3$ não tem aplicação física. Isto é particularmente interessante pois para $N \geq 5$ polinômios não possuem soluções por radicais e neste caso nem potenciais com densidade constante possuem expressões analíticas fechadas.

\subsubsection{Equação de evolução}

Em $N$ gerações trabalhamos com a equação

$$
i \frac{d}{d x}|\nu\rangle=\left[\frac{1}{2 E} U \operatorname{diag}\left(m_{1}^{2}, \ldots, m_{N}^{2}\right) U^{\dagger}+V(x) \operatorname{diag}(1,0, \ldots, 0)\right]|\nu\rangle,
$$

\footnotetext{
${ }^{1}$ Este método traz soluções para novos comportamentos de potencial, incluindo tanh $x$, $\operatorname{coth} x, \tan x$ e $\cot x$.
} 
$\operatorname{com}|\nu\rangle=\left(\begin{array}{llll}\nu_{e}=\nu_{1} & \nu_{\mu}=\nu_{2} & \cdots & \nu_{N}\end{array}\right)^{T}$ e $U$ a matriz de mistura. Assim, definindo $M^{2}=U \operatorname{diag}\left(m_{1}^{2}, \ldots, m_{N}^{2}\right) U^{\dagger}$ obtemos [204]

$$
i \frac{d}{d x}|\nu\rangle=\left[\begin{array}{ccc}
\frac{1}{2 E} M_{11}^{2}+V(x) & \cdots & \frac{1}{2 E} M_{1 N}^{2} \\
\vdots & \ddots & \vdots \\
\frac{1}{2 E} M_{N 1}^{2} & \cdots & \frac{1}{2 E} M_{N N}^{2}
\end{array}\right]|\nu\rangle .
$$

\subsubsection{Formalismo}

Por convenção adotamos $V(x)=V f\left(x / x_{0}\right)+V_{0}$. A função que dá a forma do potencial, $f$, sem dimensão. A mudança de variável $u=x / x_{0}$ fornece uma equação adimensional. Desta forma, definimos $H(u)$ por

$$
H(u)=H+\left(x_{0} V f(u)+x_{0} V_{0}\right) \operatorname{diag}(1,0, \ldots, 0),
$$

com $H=\frac{x_{0}}{2 E} M^{2}$, para obter a equação de evolução na forma $i \frac{d}{d u}|\nu\rangle=H(u)|\nu\rangle$.

Trabalhamos agora apenas com a submatriz a partir da segunda linha e segunda coluna, onde generalizaremos o resultado obtido em [204],

$$
W=\frac{x_{0}}{2 E}\left[\begin{array}{ccc}
M_{22}^{2} & \cdots & M_{2 N}^{2} \\
\vdots & \ddots & \vdots \\
M_{N 2}^{2} & \cdots & M_{N N}^{2}
\end{array}\right]
$$

assim $W=W^{\dagger}$, ou seja, existe uma matriz unitária $R$ que diagonaliza $W$, $W=R^{\dagger} D R$ com $D=\operatorname{diag}\left(\omega_{2}, \ldots, \omega_{3}\right)$, sendo todos $\omega_{j}$ reais. Em $N$ dimensões, definimos

$$
R_{N}=\left[\begin{array}{cccc}
1 & 0 & \cdots & 0 \\
0 & & & \\
\vdots & & R & \\
0 & & &
\end{array}\right]
$$

e as funções

$$
\left[\begin{array}{c}
\psi_{1}=\nu_{e} \\
\vdots \\
\psi_{N}
\end{array}\right]=R_{N}\left[\begin{array}{c}
\nu_{e} \\
\vdots \\
\nu_{N}
\end{array}\right]
$$

É interessante notar que a função de onda do neutrino eletrônico permanece inalterada.

Através de uma diagonalização mantendo a primeira linha e primeira coluna inalteradas, pelas matrizes acima definidas, obtemos a seguinte equação de 
evolução

$$
i \frac{d}{d u}|\psi\rangle=\left(\begin{array}{ccccc}
\frac{x_{0}}{2 E} M_{11}^{2}+x_{0} V f(u) & \chi_{2}^{*} & \chi_{3}^{*} & \ldots & \chi_{N}^{*} \\
\chi_{2} & \omega_{2} & 0 & \ldots & 0 \\
\chi_{3} & 0 & \omega_{3} & \ldots & 0 \\
\vdots & \vdots & \vdots & \ddots & \vdots \\
\chi_{N} & 0 & 0 & \ldots & \omega_{N}
\end{array}\right)|\psi\rangle
$$

com

$$
\chi=\left[\begin{array}{c}
\chi_{2} \\
\vdots \\
\chi_{N}
\end{array}\right]=\frac{x_{0}}{2 E} R\left[\begin{array}{c}
M_{21}^{2} \\
\vdots \\
M_{N 1}^{2}
\end{array}\right]
$$

Quando $x_{0} V_{0}=0$, a matriz $\tilde{H}=R_{N} H R_{N}^{\dagger}$ tem como autovalores as massas quadradas dos neutrinos no vácuo multiplicadas por um fator $x_{0} / 2 E$. Definimos portanto $\mu_{j}=\frac{x_{0}}{2 E} m_{j}^{2}, j=1, \ldots, N$. No caso em que $x_{0} V_{0} \neq 0$, esta solução muda para as massas dos neutrinos autoestados de massas sob efeito de densidade constante com $V(x)=V_{0}$, ou seja, $\tilde{\mu}_{j}=\frac{x_{0}}{2 E} \tilde{m}_{j}^{2}$.

O polinômio característico de $\tilde{H}$ é

$$
P_{\tilde{H}}(X)=\prod_{j=1}^{N}\left(X-\omega_{j}\right)-\sum_{j=2}^{N}\left|\chi_{j}\right|^{2} \prod_{\substack{k=2 \\ k \neq j}}^{N}\left(X-\omega_{k}\right) \equiv \prod_{j=1}^{N}\left(X-\mu_{j}\right) .
$$

Por conveniência adotamos $V_{0}=0$. Caso contrário basta trocar $\mu_{j} \rightarrow \tilde{\mu}_{j}$.

De volta à equação, definimos as funções $\psi_{11}, \psi_{22}, \ldots, \psi_{N N}$ de maneira não única através das equações

$$
\begin{aligned}
& \psi_{1}=\prod_{j=2}^{N}\left(i \frac{d}{d u}-\omega_{j}\right) \psi_{11} \\
& \psi_{j}=\chi_{j} \prod_{\substack{k=2 \\
k \neq j}}^{N}\left(i \frac{d}{d u}-\omega_{k}\right) \psi_{j j}, j=2, \ldots, N .
\end{aligned}
$$

As equações para $\psi_{2}, \ldots, \psi_{N}$ na Eq. (7.7) vão implicar em

$$
\prod_{k=2}^{N}\left(i \frac{d}{d u}-\omega_{k}\right)\left(\psi_{11}-\psi_{j j}\right)=0, \quad j=2, \ldots, N
$$

ou seja, $\psi_{j j}=\psi_{11}+$ solução homogênea, $j=2, \ldots, N$. Contudo a definição de $\psi_{11}, \ldots, \psi_{N N}$ é dada por equações não homogêneas. Para $\psi_{11}$ a equação é de ordem $N-1$ e para as restantes de ordem $N-2$. Portanto a solução mais geral ${ }^{2}$ é

\footnotetext{
${ }^{2}$ Uma equação linear possui solução única dadas as condições iniciais, e esta solução é a
} 
$\psi_{11}=\psi_{p}+\sum_{j=2}^{N} C_{j} e^{-i \omega_{j} u}$, já que $\omega_{2} \neq \ldots \neq \omega_{N}$ pois senão o problema poderia ser reduzido à uma geração inferior. Finalmente, chamando $C_{j}=K_{j j}$ encontramos as expressões analíticas $\psi_{j j}=\psi_{p}+\sum_{k=2}^{N}\left(K_{j k}-K_{k k}\right) e^{-i \omega_{k} u}$. Conclui-se assim que basta encontrar a solução particular $\psi_{p}$ para resolver o problema da equação de evolução.

Para simplificar a notação chamamos $\psi_{p} \equiv \psi$. A única equação ainda não utilizada na Eq. (7.7) é aquela para $\psi_{1}$ :

$$
\begin{gathered}
i \frac{d}{d u} \psi_{1}=\left(\omega_{1}+x_{0} V f(u)\right) \psi_{1}+\sum_{k=2}^{N} \chi_{k}^{*} \psi_{k} \\
\left(i \frac{d}{d u}-\omega_{1}-x_{0} V f(u)\right) \prod_{j=2}^{N}\left(i \frac{d}{d u}-\omega_{j}\right) \psi= \\
\quad=\sum_{j=2}^{N}\left|\chi_{j}\right|^{2} \prod_{\substack{k=2 \\
k \neq j}}^{N}\left(i \frac{d}{d u}-\omega_{j}\right) \psi \\
P_{\tilde{H}}\left(i \frac{d}{d u}\right) \psi=x_{0} V f(u) \prod_{j=2}^{N}\left(i \frac{d}{d u}-\omega_{j}\right) \psi
\end{gathered}
$$

de onde obtemos o principal resultado desse formalismo:

$$
\prod_{j=1}^{N}\left(i \frac{d}{d u}-\mu_{j}\right) \psi=x_{0} V f(u) \prod_{j=2}^{N}\left(i \frac{d}{d u}-\omega_{j}\right) \psi
$$

Conclui-se assim que esta equação é válida em $N$ gerações com o potencial agindo apenas nos neutrinos eletrônicos. Além disso, vemos que a matriz massa não precisa ser real como considerada nas Refs. [197, 204]. Finalmente, observamos que a função de onda do neutrino eletrônico permanece inalterada:

$$
\nu_{e}(u)=\psi_{1}(u)=\prod_{j=2}^{N}\left(i \frac{d}{d u}-\omega_{j}\right) \psi(u)
$$

$\operatorname{com} u=x / x_{0}$.

\subsection{Resultados exatos em duas gerações}

A equação de evolução dos neutrinos em duas gerações pode ser escrita da seguinte forma,

$$
i \frac{d}{d x}|\nu\rangle=\left(\begin{array}{cc}
V(x)-\Delta \cos 2 \theta & \Delta \sin 2 \theta \\
\Delta \sin 2 \theta & \Delta \cos 2 \theta
\end{array}\right)|\nu\rangle,
$$

onde $\Delta=\frac{\Delta m^{2}}{4 E}$ e $\Delta m^{2}=m_{2}^{2}-m_{1}^{2}$, de maneira que o formalismo desenvolvido acima possa ser facilmente aplicado. Assim temos $\omega_{2}=x_{0} \Delta \cos 2 \theta$ e $-\mu_{1}=\mu_{2}=$ $x_{0} \Delta$.

soma das soluções homogêneas linearmente independentes com uma solução particular. 
Através desse formalismo notamos que os potenciais cujas soluções são conhecidas na literatura podem ser classificados como pertencentes à três grupos distintos: constante $f(u)=1$, linear generalizado $f(u)=\frac{a_{1}+b_{1} u}{a_{2}+b_{2} u}$ e exponencial generalizado $f(u)=\frac{a_{1}+b_{1} e^{-u}}{a_{2}+b_{2} e^{-u}}$. O potencial constante abrange o vácuo quando é nulo, e quando não é nulo pode auxiliar no entendimento do efeito $M S W[37,38]$ como veremos adiante. Os potenciais trigonométricos estão contidos dentro do caso exponencial na troca $x \rightarrow i x$.

Todas as soluções $\nu_{e}\left(x, x_{1}\right)$ nesta seção serão dadas considerando as condições iniciais $\nu_{e}\left(x_{1}\right)=0$ e $\nu_{X}\left(x_{1}\right)=1$, onde $X=\mu, \tau$ ou uma mistura destes. Desta forma, a probabilidade de transição de sabor entre os pontos $x_{1}$ e $x$ é $P\left(\nu_{X} \rightarrow \nu_{e} ; x_{1}, x\right)=\left|\nu_{e}\left(x_{1}, x\right)\right|^{2}$. Evidentemente, no caso da propagação no vácuo podemos substituir $\nu_{e}$ por qualquer outro sabor.

\subsubsection{Caso constante}

O potencial constante $V(x)=V$ é obtido com $f(u)=1$ e $V_{0}=0$ (ou analogamente $f=0$ e $V_{0}=V$ ). Desta forma, a partir da Eq. (7.15) é evidente que as soluções são $\psi_{p}^{(1,2)}(u)=e^{-i r_{1,2} u}$, onde $r_{1,2}$ são as raízes de

$$
\left(r-\mu_{1}\right)\left(r-\mu_{2}\right)-x_{0} V\left(r-\omega_{2}\right)=0 .
$$

Essas raízes são os autovalores de massa dos neutrinos dentro de um meio de densidade constante. Como a Eq. (7.17) já possui subtrações de termos proporcionais à identidade, não vale a pena escrever a solução completa aqui, podemos assim subtrair os seus termos proporcionais à identidade que resultam numa fase comum à ambas soluções. Obtemos portanto como resultado as duas soluções linearmente independentes

$$
\nu_{e}^{1,2}(x)=e^{ \pm i x \Delta \sqrt{\left(\frac{V}{2 \Delta}-\cos 2 \theta\right)^{2}+\sin ^{2} 2 \theta}} .
$$

Ao aplicar as condições iniciais, chegamos à

$$
\nu_{e}\left(x_{1}, x\right)=-i \frac{\sin 2 \theta}{C} \sin \Delta C\left(x-x_{1}\right),
$$

em que $C=\sqrt{\left(\frac{V}{2 \Delta}-\cos 2 \theta\right)^{2}+\sin ^{2} 2 \theta}$.

Estas soluções, $\nu_{e}^{(1,2)}$, são as funções autoestados da hamiltoniana, chamadas de autoestados de massa na matéria, diferente dos autoestados de massa no vácuo. Delas extraimos os seguintes parâmetros efetivos na matéria: $\sin 2 \tilde{\theta}=$ $\sin 2 \theta / C$ e $\Delta \tilde{m}^{2}=C \Delta m^{2}$. Elas não se misturam durante todo o processo de evolução. 


\section{Vácuo}

No vácuo, $V=0 \Rightarrow C=1$, a probabilidade de transição é

$$
P\left(\nu_{X} \rightarrow \nu_{Y} ; x_{1}, x\right)=\sin ^{2} 2 \theta \sin ^{2} \Delta\left(x-x_{1}\right) .
$$

cuja expressão já foi usada e comentada no Capítulo 4. Ressaltamos novamente apenas que o ângulo de mistura delimita a profundidade de oscilação, sendo tão pequena quanto $\sin ^{2} 2 \theta$, e máxima quando $\theta=\pi / 4$. Além disso, a média sobre a energia num feixe não monocromático é a metade da profundidade: $\left\langle P_{X Y}\right\rangle=$ $\sin ^{2} 2 \theta / 2$.

\section{Efeito MSW}

Se a matéria através da qual o feixe se propaga contiver elétrons à uma densidade constante, e diferente de zero, $V$ é chamado de um potencial constante não nulo. Isso resulta em

$$
P\left(\nu_{X} \rightarrow \nu_{e} ; x_{1}, x\right)=\frac{\sin ^{2} 2 \theta}{C^{2}} \sin ^{2} \Delta C\left(x-x_{1}\right)
$$

O ângulo efetivo, que define a profundidade de oscilação, pode atingir o valor máximo $\tilde{\theta}=\pi / 4$ mesmo se $\theta$ for pequeno, contanto que $V=2 \Delta \cos 2 \theta$ seja satisfeito.

A Eq. (7.22) é a solução exata para um potencial com densidade constante. Entretanto, a variação do valor de $\mathrm{V}$ nesta equação nos ajuda à compreender melhor o efeito de matéria de um potencial arbitrário. De fato, a profundidade de oscilação definida por

$$
\sin ^{2} 2 \tilde{\theta}=\frac{\sin ^{2} 2 \theta}{(A(x)-\cos 2 \theta)^{2}+\sin ^{2} 2 \theta}
$$

onde $A(x)=\frac{2 E V(x)}{\Delta m^{2}}$, é amplificada pelo potencial dentro de uma certa região, como vemos na Figura 7.1. Esta região é chamada de zona de ressonância. Definimos a largura (em distância) de ressonância, $\delta x_{R}$, correspondendo à região onde $\sin ^{2} 2 \tilde{\theta} \geq \frac{1}{2}$, em torno do ponto de ressonância $A_{R}=\cos 2 \theta$. Como $\sin ^{2} 2 \tilde{\theta}=$ $\frac{1}{2}$ fornece $A=\cos 2 \theta \pm \sin 2 \theta$, então $\delta A_{R}=2 \sin 2 \theta \mathrm{e}$

$$
\delta x_{R} \simeq \frac{\delta A_{R}}{\left|\frac{d}{d x} A\right|_{R}}=\frac{2 \tan 2 \theta}{h_{R}}
$$

onde definimos a altura de ressonância $h_{R} \equiv|\dot{A} / A|_{R}$. Este efeito ressonante é conhecido como efeito $M S W[37,38]$. Além disso, uma vez que o sinal de $V(x)$ 
é conhecido, a ressonância só pode ocorrer para um dado sinal de $\Delta m^{2} \cos 2 \theta$. Portanto a simetria presente na análise de oscilações no vácuo é quebrada pelo efeito de matéria. Também é interessante ver que se o ponto de ressonância é alcançado para dois sabores de neutrinos, este ponto não pode ser obtido para antineutrinos do mesmo sabor e vice-versa.

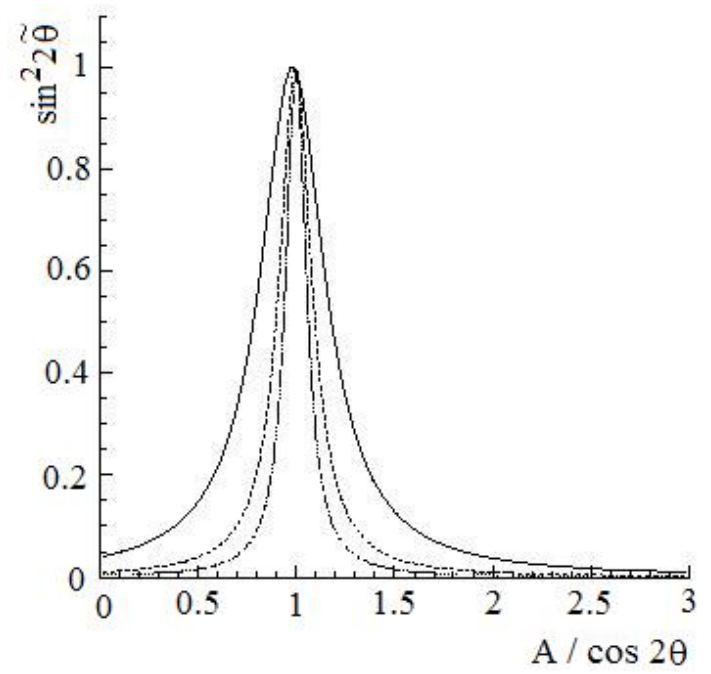

Figura 7.1: Profundidade de oscilação, $\sin ^{2} 2 \theta$, em função de $A / \cos 2 \theta$ para três valores diferentes do ângulo de mistura no vácuo: $\sin ^{2} 2 \theta=0,04$ (linha sólida), $\sin ^{2} 2 \theta=0,01$ (linha intermediária) e $\sin ^{2} 2 \theta=0,004$ (linha pontilhada no interior).

O interessante na ressonância não é apenas o fato de que ela aumenta a probabilidade de transição localmente, mas se considerarmos a média de um feixe de neutrinos percebemos que ela aumenta na mesma proporção: $\left\langle P_{X Y}\right\rangle=\frac{\sin ^{2} 2 \theta}{2 C^{2}}$. De fato, supondo que o ângulo de mistura no vácuo seja pequeno, $\theta \simeq 0$, ao passar por uma região ressonante, uma grande quantidade de neutrinos deverá mudar de sabor $\nu_{X} \rightarrow \nu_{Y}$. Ao voltar para a oscilação no vácuo, não ocorrerá mais tanta transição, mantendo assim os neutrinos no novo sabor.

\subsubsection{Caso exponencial generalizado}

A Eq. (7.15) se parece muito com a equação diferencial das funções hipergeométricas generalizadas $[205,206]$. Para transformar uma na outra o potencial deve apresentar um comportamento exponencial, sendo o caso mais geral $f(u)=$ $\frac{a_{1}+b_{1} e^{-u}}{a_{2}+b_{2} e^{-u}}$.

De fato, introduzindo a função exponencial generalizada na Eq. (7.15) temos

$$
\left(i \frac{d}{d u}-\mu_{1}\right)\left(i \frac{d}{d u}-\mu_{2}\right) \psi=x_{0} V \frac{a_{1}+b_{1} e^{-u}}{a_{2}+b_{2} e^{-u}}\left(i \frac{d}{d u}-\omega_{2}\right) \psi
$$


Rearranjando os termos encontramos

$$
\begin{gathered}
{\left[\left(i \frac{d}{d u}-\mu_{1}\right)\left(i \frac{d}{d u}-\mu_{2}\right)-x_{0} V \frac{a_{1}}{a_{2}}\left(i \frac{d}{d u}-\omega_{2}\right)\right] \psi+} \\
\frac{b_{2}}{a_{2}} e^{-u}\left[\left(i \frac{d}{d u}-\mu_{1}\right)\left(i \frac{d}{d u}-\mu_{2}\right)-x_{0} V \frac{b_{1}}{b_{2}}\left(i \frac{d}{d u}-\omega_{2}\right) \psi\right]=0
\end{gathered}
$$

de onde vemos que é importante introduzir os parâmetros

$$
\begin{aligned}
& \tilde{\mu}_{1,2}^{a}=\frac{x_{0} V}{2} \frac{a_{1}}{a_{2}} \mp x_{0} \Delta \sqrt{\left(\cos 2 \theta-\frac{V}{2 \Delta} \frac{a_{1}}{a_{2}}\right)^{2}+\sin ^{2} 2 \theta} \\
& \tilde{\mu}_{1,2}^{b}=\frac{x_{0} V}{2} \frac{b_{1}}{b_{2}} \mp x_{0} \Delta \sqrt{\left(\cos 2 \theta-\frac{V}{2 \Delta} \frac{b_{1}}{b_{2}}\right)^{2}+\sin ^{2} 2 \theta}
\end{aligned},
$$

para chegar à forma

$$
\left[\left(i \frac{d}{d u}-\tilde{\mu}_{1}^{a}\right)\left(i \frac{d}{d u}-\tilde{\mu}_{2}^{a}\right)+\frac{b_{2}}{a_{2}} e^{-u}\left(i \frac{d}{d u}-\tilde{\mu}_{1}^{b}\right)\left(i \frac{d}{d u}-\tilde{\mu}_{2}^{b}\right)\right] \psi=0 .
$$

A equação hipergeométrica generalizada em duas gerações é justamente a equação da função hipergeométrica. Para encontrar esta equação basta fazer a mudança de variável $z=-\frac{b_{2}}{a_{2}} e^{-u}$, o que significa que $\frac{d}{d u}=-z \frac{d}{d z}$. Isto conduz à

$$
\left[\left(z \frac{d}{d z}-i \tilde{\mu}_{1}^{a}\right)\left(z \frac{d}{d z}-i \tilde{\mu}_{2}^{a}\right)+z\left(z \frac{d}{d z}-i \tilde{\mu}_{1}^{b}\right)\left(z \frac{d}{d z}-i \tilde{\mu}_{2}^{b}\right)\right] \psi=0
$$

cujas duas soluções linearmente independentes são

$$
\begin{aligned}
& \psi^{(1)}(z)=z^{i \tilde{\mu}_{1}^{a}}{ }_{2} F_{1}\left(i \tilde{\mu}_{1}^{a}-i \tilde{\mu}_{1}^{b}, i \tilde{\mu}_{1}^{a}-i \tilde{\mu}_{2}^{b} ; 1+i \tilde{\mu}_{1}^{a}-i \tilde{\mu}_{2}^{a} ; z\right) \\
& \psi^{(2)}(z)=z^{i \tilde{\mu}_{2}^{a}}{ }_{2} F_{1}\left(i \tilde{\mu}_{2}^{a}-i \tilde{\mu}_{1}^{b}, i \tilde{\mu}_{2}^{a}-i \tilde{\mu}_{2}^{b} ; 1+i \tilde{\mu}_{2}^{a}-i \tilde{\mu}_{1}^{a} ; z\right)
\end{aligned} .
$$

Definindo os parâmetros

$$
\begin{gathered}
\lambda_{a}=i x_{0} V \frac{a_{1}}{a_{2}}, \lambda_{b}=i x_{0} V \frac{b_{1}}{b_{2}} \\
\nu_{a}=i x_{0} \Delta \sqrt{\left(\frac{V a_{1}}{2 \Delta a_{2}}-\cos 2 \theta\right)^{2}+\sin ^{2} 2 \theta} \\
\nu_{b}=i x_{0} \Delta \sqrt{\left(\frac{V b_{1}}{2 \Delta b_{2}}-\cos 2 \theta\right)^{2}+\sin ^{2} 2 \theta}
\end{gathered}
$$

e fazendo as substituições

$$
i \tilde{\mu}_{1,2}^{a}=\frac{\lambda_{a}}{2} \mp \nu_{a} \quad \text { e } \quad i \tilde{\mu}_{1,2}^{b}=\frac{\lambda_{b}}{2} \mp \nu_{b}
$$




$$
\text { e } \quad i \omega_{2}=\frac{\nu_{b}^{2}-\nu_{a}^{2}+\left(\lambda_{a}^{2}-\lambda_{b}^{2}\right) / 4}{\lambda_{a}-\lambda_{b}}
$$

percebemos que

$$
\psi^{(1,2)}(z)=z^{\frac{\lambda_{a}}{2} \mp \nu_{a}}{ }_{2} F_{1}\left(\nu_{b} \mp \nu_{a}+\frac{\lambda_{a}-\lambda_{b}}{2},-\nu_{b} \mp \nu_{a}+\frac{\lambda_{a}-\lambda_{b}}{2} ; 1 \mp 2 \nu_{a} ; z\right),
$$

ou seja, $\psi^{(1)} \leftrightarrow \psi^{(2)}$ sob $\nu_{a} \leftrightarrow-\nu_{a}$.

Vamos agora voltar à função de onda do neutrino eletrônico, lembrando que $\nu_{e}=\left(i \frac{d}{d u}-\omega_{2}\right) \psi$ ou com a mudança de variável $\nu_{e}^{(1,2)}=\left(z \frac{d}{d z}-i \omega_{2}\right) \psi^{(1,2)}$. Sendo assim, obtemos

$$
\nu_{e}^{(1,2)}(z)=z^{\frac{\lambda a}{2} \mp \nu_{a}}\left(\frac{\lambda_{a}}{2} \mp \nu_{a}-\frac{\nu_{b}^{2}-\nu_{a}^{2}+\left(\lambda_{a}^{2}-\lambda_{b}^{2}\right) / 4}{\lambda_{a}-\lambda_{b}}+z \frac{d}{d z}\right){ }_{2} F_{1}
$$

onde o argumento da função hipergeométrica foi omitido para abreviar a notação. Para simplificar ainda mais os cálculos, renomeamos as váriaveis com as letras usuais das funções hipergeométricas

$$
a=\nu_{b}-\nu_{a}+\frac{\lambda_{a}-\lambda_{b}}{2}, \quad b=-\nu_{b}-\nu_{a}+\frac{\lambda_{a}-\lambda_{b}}{2} \quad \text { e } \quad c=1-2 \nu_{a} .
$$

Isto conduz ao resultado

$$
\nu_{e}^{(1)}(z)=z^{\frac{\lambda_{a}}{2}-\nu_{a}}\left(\frac{a b}{a+b+1-c}+z \frac{d}{d z}\right){ }_{2} F_{1}(a, b ; c ; z) .
$$

Após algumas manipulações elementares, as propriedades da função hipergeométrica [207, 208] nos leva à

$$
\nu_{e}^{(1)} \propto z^{\frac{\lambda a}{2}-\nu_{a}}(1-z){ }_{2} F_{1}(a+1, b+1 ; c ; z) .
$$

De fato, vamos demonstrar este resultado a partir da Eq. (7.36). Por definição de equação hipergeométrica, ${ }_{2} F_{1}(a, b ; c ; z)$ é solução de

$$
\left[z(1-z) \frac{d^{2}}{d z^{2}}+[c-(a+b+1) z] \frac{d}{d z}-a b\right]{ }_{2} F_{1}=0,
$$

ou analiticamente

$$
{ }_{2} F_{1}(a, b ; c ; z)=\sum_{k=0}^{\infty} \frac{(a)_{k}(b)_{k}}{(c)_{k}} \frac{z^{k}}{k !}
$$

onde $(a)_{k}=\frac{\Gamma(a+k)}{\Gamma(a)}=a(a+1) \cdots(a+k-1)$. Utilizando a Eq. (7.38) na Eq.(7.36) 
encontramos

$$
\begin{gathered}
\nu_{e}^{(1)}(z) \propto z^{\frac{\lambda a}{2}-\nu_{a}}\left[z(1-z) \frac{d^{2}}{d z^{2}}+c(1-z) \frac{d}{d z}\right]{ }_{2} F_{1}(a, b ; c ; z) \\
=z^{\frac{\lambda a}{2}-\nu_{a}}(1-z) \frac{d}{d z}\left[z \frac{d}{d z}+(c-1)\right]_{2} F_{1}(a, b ; c ; z)
\end{gathered} .
$$

Agora basta aplicar a definição da Eq. (7.39) para ver que

$$
\begin{aligned}
\frac{d}{d z}\left[z \frac{d}{d z}+(c-1)\right]_{2} F_{1}(a, b ; c ; z) & =(c-1) \frac{d}{d z}{ }_{2} F_{1}(a, b ; c-1 ; z) \\
& =a b_{2} F_{1}(a+1, b+1 ; c ; z)
\end{aligned} .
$$

Com a finalidade de comparar o resultado obtido por este método com os conhecidos na literatura façamos a mudança $y=z /(z-1)$. Portanto o par de soluções é

$$
\nu_{e}^{(1,2)}(y)=y^{\frac{\lambda a}{2} \pm \nu_{a}}(1-y)^{-\frac{\lambda_{b}}{2}-\nu_{b}} \quad{ }_{2} F_{1}(\alpha, \beta ; \gamma ; y)
$$

em que $\alpha=\mp \nu_{a}-\nu_{b}-\lambda_{a} / 2+\lambda_{b} / 2, \beta=\mp \nu_{a}-\nu_{b}+\lambda_{a} / 2-\lambda_{b} / 2+1$ e $\gamma=1 \mp 2 \nu_{a}$.

Entretanto, a solução aqui apresentada vai além dos dois exemplos conhecidos [203, 199, 200, 201, 202] pelo fato da forma mais geral do potencial que utilizamos ter 3 parâmetros livres, assim ela pode ajustar mais potenciais, por exemplo uma densidade que varie $\operatorname{como} \operatorname{coth} \frac{u}{2}$.

\section{Potencial em tangente hiperbólica}

Na Ref. [203] é apresentada a solução para o potencial do tipo tangente hiperbólica equivalente à $f(u)=1+\tanh (u / 2)$. Neste caso, $a_{1}=2, a_{2}=1, b_{1}=$ $0, b_{2}=1$ e fazendo a alteração $x_{0} \rightarrow x_{0} / 2$ obtemos os parâmetros $\lambda_{a}=-2 \lambda$, $\lambda_{b}=0, \nu_{a}=\nu$ e $\nu_{b}=-\mu$ introduzidos em [203].

Portanto, as soluções obtidas aqui são as mesmas da Ref. [203]:

$$
\nu_{e}^{(1,2)}(y)=y^{\lambda \mp \nu}(1-y)^{\mu}{ }_{2} F_{1}(\mu \mp \nu+\lambda, \mu \mp \nu-\lambda+1 ; 1 \mp 2 \nu ; y) .
$$

\section{Potencial variando exponencialmente}

Nas Refs. [199, 200, 201, 202] encontram-se várias resoluções para um potencial equivalente à $f(u)=e^{-u}$. Neste caso $a_{1}=0, b_{1}=1, a_{2}=1$ e devemos tomar o limite $b_{2} \rightarrow 0$, pois este parâmetro aparece em denominadores. 
Calculemos portanto os parâmetros da solução neste limite:

$$
\begin{aligned}
& \lim _{b_{2} \rightarrow 0} \quad \nu_{b}-\nu_{a}+\frac{\lambda_{a}}{2}-\frac{\lambda_{b}}{2}+1= \\
& \lim _{b_{2} \rightarrow 0} \quad \nu_{b}-\mu-\frac{\lambda_{b}}{2}+1= \\
& \lim _{b_{2} \rightarrow 0} \quad i x_{0} \Delta \sqrt{\left(\cos 2 \theta-\frac{V}{2 \Delta} \frac{1}{b_{2}}\right)^{2}+\sin ^{2} 2 \theta}-\mu-\frac{i x_{0} V}{2 b_{2}}+1= \\
& \lim _{b_{2} \rightarrow 0} i x_{0} \Delta\left(\frac{V}{2 \Delta b_{2}}\right)\left[1+\frac{b_{2}}{2}\left(\frac{-2 \cos 2 \theta V / 2 \Delta}{(V / 2 \Delta)^{2}}\right)+O\left(b_{2}^{2}\right)\right]-\mu-\frac{i x_{0} V}{2 b_{2}}+1= \\
& -i x_{0} \Delta \cos 2 \theta-\mu+1 \\
& -\mu-\lambda+\frac{1}{2}
\end{aligned}
$$

já que $\nu_{a}=\mu$ e $\lambda_{a}=0$. E o outro parâmetro que devemos considerar é

$$
I(n)=\frac{\Gamma\left(-\nu_{b}-\mu-\frac{\lambda_{b}}{2}+1+n\right)}{\Gamma\left(-\nu_{b}-\mu-\frac{\lambda_{b}}{2}+1\right)}\left(-b_{2}\right)^{n},
$$

o qual não é difícil de ver que o limite é

$$
\lim _{b_{2} \rightarrow 0} I(n)=\left(i x_{0} V\right)^{n}
$$

Portanto a função hipergeométrica se torna uma hipergeométrica confluente com $z \rightarrow z=i x_{0} V e^{-u}$

$$
{ }_{2} F_{1}(a+1, b+1 ; c ; z) \rightarrow \quad{ }_{1} F_{1}\left(-\mu-\lambda+\frac{1}{2} ; 1-2 \mu ; z\right),
$$

quando $b_{2} \rightarrow 0$.

Desta forma encontramos os resultados apresentados nas Refs. [200, 201] utilizando a definição de funções de Whittaker:

$$
\nu_{e}^{(1,2)}(z)=e^{z^{\prime} / 2} z^{1 / 2} M_{\lambda, \mp \mu}(z),
$$

onde os fatores multiplicativos representam apenas uma fase comum.

Como podemos utilizar qualquer duas funções de Whittaker linearmente independentes, escolhemos $W_{ \pm \lambda}( \pm z)$ correspondentes à mesma equação. Aplicando as condições iniciais não é difícil de se chegar ao seguinte resultado final:

$$
\nu_{e}(z)=\left(z z_{1}\right)^{-\frac{1}{2}}\left(-i \Delta x_{0} \sin 2 \theta\right) e^{i \pi \lambda}\left(W_{-\lambda, \mu}\left(-z_{1}\right) W_{\lambda, \mu}(z)-W_{\lambda, \mu}\left(z_{1}\right) W_{-\lambda, \mu}(-z)\right)
$$

lembrando que o Wronskiano da função de Whittaker é $W\left[W_{-\lambda, \mu}(-z) ; W_{\lambda, \mu}(z)\right]=$ $-e^{-i \pi \lambda}$. 


\section{Potenciais trigonométricos}

Com pequenas modificações nas variáveis $(u \rightarrow i u)$ e nos parâmetros $\left(\lambda_{a, b} \rightarrow\right.$ $-i \lambda_{a, b}$ e $\left.\nu_{a, b} \rightarrow-i \nu_{a, b}\right)$, as solução para potenciais trigonométricos como $f(u)=$ $\tan \frac{u}{2}$ ou cot $\frac{u}{2}$ também podem ser encontrados pela Eq. (7.42).

Deve-se entretanto notar que neste caso o domínio de aplicabilidade fica restringido e o fato de não conhecermos aplicação física destes tipos de potenciais não nos motivou um estudo mais profundo destas soluções.

\subsubsection{Caso linear generalizado}

No caso linear, cuja forma mais geral é $f(u)=\frac{a_{1}+b_{1} u}{a_{2}+b_{2} u}$, nossa análise será no caminho inverso daquela feita para o caso exponencial. De fato, uma mudança de variável reduz o caso geral à $1 / x$, se $b_{2} \neq 0$, ou ao caso linear se $b_{2}=0$.

Em duas gerações existem duas maneiras de se proceder para encontrar a solução. Acontece que a Eq. (7.15) já é uma equação hipergeométrica confluente para tal potencial linear generalizado, porém este método de resolução não se aplicaria à 3 gerações. Como observado na Ref. [197], a vantagem de um potencial linear é que podemos usar a representação de Fourier (a mais intuitiva na resolução da Eq. (7.15)) para obter uma equação diferencial linear homogênea de primeira ordem e portanto expressar a solução pelo menos por uma integral.

Portanto é evidente que em duas gerações devemos encontrar como solução a representação integral de alguma função hipergeométrica confluente. Isto já não é verdade em três gerações, e a solução será apenas dada por uma representação integral.

No que segue primeiro encontramos a solução de um potencial que varia linearmente com a distância, $V(x) \propto x$. Isto ocorre se $b_{2}=0$ e fazemos $V_{0}=\frac{a_{1}}{a_{2}}$ e $f(u)=u$, absorvendo o termo $\frac{b_{1}}{a_{2}}$ em $V$. Na segunda parte $b_{2} \neq 0$, mas para simplificar $u \rightarrow u-\frac{a_{2}}{b_{2}}$ restando apenas a análise de $V(x) \propto 1 / x$, ou seja, o potencial variando como uma hipérbole.

\section{Potencial linear}

Evidentemente o estudo aqui realizado tem como objetivo final comparar o resultado obtido através do formalismo proposto com as soluções apresentadas na literatura para um potencial $V(x) \propto x$ [197]. Desta forma, para facilitar a comparação subtraimos da Eq. (7.17) o termo proporcional a identidade $\Delta \cos 2 \theta$. Além disso, fazemos a mudança $z=e^{i \frac{\pi}{4}} \sqrt{V / x_{0}}\left(x-\frac{2 \Delta \cos 2 \theta}{V} x_{0}\right)$ de modo que $\mu_{1,2}= \pm e^{-i \frac{\pi}{4}} \Delta \sqrt{x_{0} / V}$ e $\omega_{2}=0$. 
A Eq. (7.15) neste caso torna-se

$$
\left(i \frac{d}{d z}-\mu_{1}\right)\left(i \frac{d}{d z}-\mu_{2}\right) \psi=e^{-i \frac{\pi}{2}} z\left(i \frac{d}{d z}-\omega_{2}\right) \psi
$$

Definimos agora a transformada de Fourier como

$$
\begin{aligned}
\psi(z) & =\int_{-\infty}^{+\infty} d t e^{-i t z} \phi(t) \\
\phi(t) & =\frac{1}{2 \pi} \int_{-\infty}^{+\infty} d z e^{i t z} \psi(z)
\end{aligned}
$$

de modo que a Eq. (7.50) se transforma em

$$
\left(t-\mu_{1}\right)\left(t-\mu_{2}\right) \phi=-i\left(-i \frac{d}{d t}\right)\left(t-\omega_{2}\right) \phi
$$

Como $\omega_{2}=0$ e $\nu_{e}(z)=i \frac{d}{d z} \psi=\int_{-\infty}^{+\infty} d t e^{-i t z} t \phi(t)$, chamamos $\varphi(t)=t \phi(t) \mathrm{e}$ introduzimos a notação $\mu=\mu_{2}=-\mu_{1}$ para obter

$$
\frac{\left(t^{2}-\mu^{2}\right)}{t} \varphi=-\frac{d}{d t} \varphi \Rightarrow \varphi(t)=\varphi_{0} e^{-t^{2} / 2}|t|^{\mu^{2}} .
$$

Apesar de aparentar a obtenção de apenas uma solução, a Eq. (7.53) indica singularidade em $t=0$. Isto significa que em uma única expressão temos duas soluções, uma para $t>0$ e outra para $t<0$, cuja expressão analítica é dada sob o sinal de integração pela transformada de Fourier:

$$
\begin{aligned}
& \nu_{e}^{(1)}(z)=\int_{0}^{\infty} d t e^{-i t z} e^{t^{2} / 2}|t|^{\mu^{2}}=\int_{0}^{\infty} d t e^{-i t z} e^{t^{2} / 2} t^{-i p_{o}} \mathrm{e} \\
& \nu_{e}^{(2)}(z)=\int_{-\infty}^{0} d t e^{-i t z} e^{t^{2} / 2}|t|^{\mu^{2}}=\int_{0}^{\infty} d t e^{i t z} e^{t^{2} / 2} t^{-i p_{o}}
\end{aligned}
$$

onde introduzimos os parâmetros utilizados na Ref. [198] $p=-i p_{0}$ e $\sqrt{p_{0}}=$ $\Delta \sqrt{x_{0} / V}$. É fácil ver que $\nu_{e}^{(2)}(z)=\nu_{e}^{(1)}(-z)$. Pela Ref. [208] as funções de Weber podem ser definidas por

$$
D_{p^{\prime}}(z)=\frac{e^{-z^{2} / 4}}{\Gamma\left(-p^{\prime}\right)} \int_{0}^{\infty} d x e^{-x z-x^{2} / 2-p^{\prime}-1}
$$

cuja condição $\operatorname{Re}\left[p^{\prime}\right]<0$ é satisfeita na nossa solução colocando $-p^{\prime}-1=p$, de onde verificamos que as soluções encontradas são as funções de Weber a menos 
de uma fase, a saber $e^{-z^{2} / 4}$ :

$$
\nu_{e}^{(1)}(z) \propto D_{-p-1}(i z) \quad \text { e } \quad \nu_{e}^{(2)}(z) \propto D_{-p-1}(-i z)
$$

Novamente mudamos a escolha das funções linearmente independentes utilizadas para $D_{p}(z)$ e $D_{p}(-z)$ e lembramos que o Wronskiano das funções de Weber é $W\left[D_{p}(z) ; D_{p}(-z)\right]=\frac{-\sqrt{2 \pi}}{\Gamma(-p)}$, para chegar à forma final da solução aplicando as condições iniciais:

$$
\nu_{e}(z)=e^{-i \frac{3 \pi}{4}} \Gamma\left(i p_{0}\right) \sqrt{\frac{p_{0}}{2 \pi}}\left(D_{p}\left(-z_{1}\right) D_{p}(z)-D_{p}\left(z_{1}\right) D_{p}(-z)\right)
$$

\section{Caso de uma hipérbole}

Dos casos conhecidos, falta apenas encontrar a solução para um potencial $V(x) \propto$ $1 / x$. Assim como $V(x) \propto x$, esse potencial não é encontrado na natureza. A solução dele foi apresentada com o objetivo de investigar o comportamento de soluções na região do potencial que varia não adiabaticamente com a posição.

Não vamos aqui refazer todo o cálculo apresentado no caso linear, pois o método de resolução é o mesmo. As mudanças de variáveis feitas nesse caso para comparação com [160] são $z=-i 2 \Delta x_{0} u, \mu^{\prime}=\frac{ \pm \mu_{1,2}}{2 x_{0} \Delta}=\frac{1}{2}$ e $\omega^{\prime}=\frac{\omega_{2}}{2 x_{0} \Delta}=\frac{\cos 2 \theta}{2}$. Deve-se ainda introduzir os parâmetros da Ref. [160] onde $i x_{0} V\left(\frac{\mu^{\prime}-\omega^{\prime}}{2 \mu^{\prime}}\right)-1=$ $\mu+\lambda-\frac{3}{2}$ e $i x_{0} V\left(\frac{\mu^{\prime}+\omega^{\prime}}{2 \mu^{\prime}}\right)-1=\mu-\lambda-\frac{3}{2}$ levando à conclusão que $\sin ^{2} \theta=\frac{\mu+\lambda-1 / 2}{2 \mu-1}$ e $\cos ^{2} \theta=\frac{\mu-\lambda-1 / 2}{2 \mu-1}$. Com estes parâmetros e utilizando a transformada de Fourier definida nas Eqs. (7.54) e (7.55) devemos obter três soluções referentes às duas singularidades. Como a equação diferencial neste caso é de ordem 2, apenas duas destas soluções são linearmente independentes, então a idéia é trabalhar com duas delas e no final mostrar que a terceira é de fato combinação linear dessas duas.

Devido à posição invertida de $x$ em $1 / x$ em relação ao potencial linear, as singularidades se encontram em $\pm \mu^{\prime}$ e não em $\omega_{2}$ como anteriormente. Intuitivamente, as duas soluções mais simples para se estudar devem ser aquelas referentes aos domínios $t<-\mu^{\prime}$ e $t>\mu^{\prime}$ pela simetria em torno de 0. De fato, após um longo cálculo obtemos

$$
\nu_{e}^{(1,2)}(z)=e^{ \pm \frac{z}{2}} \int_{0}^{\infty} d t\left(t+\frac{\mu \pm \lambda-\frac{1}{2}}{2 \mu-1}\right)(1+t)^{\mu \mp \lambda-\frac{1}{2}} t^{\mu \pm \lambda-\frac{3}{2}} e^{ \pm z t}
$$

ou seja, $\nu_{e}^{(1)}(z) \leftrightarrow \nu_{e}^{(2)}(z)$ sob a troca de $z \leftrightarrow-z$ e $\lambda \leftrightarrow-\lambda$.

Olhemos então apenas para $\nu_{e}^{(2)}(z)$. Se queremos mostrar que ela é uma 
função de Whittaker como na Ref. [160] devemos utilizar sua representação integral, obtendo

$$
\nu_{e}^{(2)}(z)=z^{-\mu} \frac{\Gamma\left(\mu-\lambda+\frac{1}{2}\right)}{2 \mu-1}\left[(2 \mu-1) W_{\lambda-\frac{1}{2}, \mu-\frac{1}{2}}(z)+z^{\frac{1}{2}} W_{\lambda, \mu-1}(z)\right] .
$$

Agora utilizamos as relações de recorrência [206, 208] para mostrar que

$$
\nu_{e}^{(1,2)}(z)=\frac{\Gamma\left(\mu \mp \lambda+\frac{1}{2}\right)}{2 \mu-1} z^{-\mu} z^{\frac{1}{2}} W_{\mp \lambda, \mu}(\mp z)
$$

de forma que as soluções são realmente duas funções de Whittaker linearmente independentes e que coincidem com a solução da Ref. [160].

A terceira solução, $\nu_{e}^{(3)}(z)$, com uma mudança de variável pode ser escrita como

$$
\nu_{e}^{(3)}(z)=\int_{-1}^{1} d t(t-\cos 2 \theta)(1-t)^{\mu+\lambda-\frac{3}{2}}(1+t)^{\mu-\lambda-\frac{3}{2}} e^{z t / 2},
$$

que após um tedioso cálculo e uso de fórmulas de recorrência pode ser mostrada proporcional à função de Whittaker $\nu_{e}^{(3)}(z) \propto z^{-\mu+\frac{1}{2}} M_{\lambda, \mu}(z)$, portanto linearmente dependente.

Desconsiderando as fases comuns e aplicando as condições iniciais chegamos ao resultado final:

$$
\nu_{e}(z)=\frac{-\sin 2 \theta}{2} e^{\pi x_{0} V \cos 2 \theta / 2}\left(W_{-\lambda, \mu}\left(-z_{1}\right) W_{\lambda, \mu}(z)-W_{\lambda, \mu}\left(z_{1}\right) W_{-\lambda, \mu}(-z)\right) .
$$

\subsection{Resultados exatos em três gerações}

Partimos agora para o estudo da equação de evolução em três gerações. É evidente desde já que os cálculos realizados neste caso devem ser mais extensos e complicados. Entretanto, como vimos em duas gerações, o formalismo proposto é sistemático e por isso pode ser aplicado ao caso de três gerações da mesma forma. Isso garante que mesmo que de maneira tediosa encontraremos soluções para os mesmos tipos de potenciais.

Em três gerações é necessário diagonalizar uma matriz $2 \times 2$ para encontrar os valores de $\omega_{2}$ e $\omega_{3}$. Além disso, os valores $\mu_{1,2,3}$ não são tão simples neste caso por serem as raízes de um polinômio de grau 3, cuja solução analítica é obtida pela fórmula de Cardan, diferentemente do simples cálculo em duas famílias.

Como a principal parte das contas do formalismo já foi realizada em duas gerações, nesta seção apresentamos somente as diferenças e dificuldades encontradas 
em três gerações.

Além disso, embora devêssemos encontrar $P_{e \mu}$ e $P_{\mu \tau}$ para obter a expressão analítica de todas as soluções, o que significa encontrar ambos $\nu_{e}(x), \nu_{\mu}(x) \mathrm{e}$ $\nu_{\tau}(x)$, no intuito de simplificar o estudo olhamos apenas para $\nu_{e}(x)$ que não necessita da rotação $R_{N}$.

\subsubsection{Caso constante}

É simples de ver, através de 7.2.1, que as soluções no caso de um potencial constante são $\psi^{(1,2,3)}(u)=e^{-i r_{1,2,3} u}$, onde $r_{1,2,3}$ são as três raízes de

$$
\left(r-\mu_{1}\right)\left(r-\mu_{2}\right)\left(r-\mu_{3}\right)-x_{0} V\left(r-\omega_{2}\right)\left(r-\omega_{3}\right)=0
$$

Para obter a expressão analítica das probabilidades de oscilação de neutrinos é necessário encontrar as autofunções, além dos autovalores, o que aumenta significativamente a quantidade de cálculos realizados.

Tendo em vista a quantidade de operações a serem realizadas num cálculo direto de tais autovalores e autofunções, dois métodos alternativos foram propostos para obtenção das expressões analíticas para as probabilidades de oscilação. Devido à sua importância, todos os métodos conhecidos que tratam de um potencial constante são discutidos em 7.4.

\section{Vácuo}

Obviamente, no vácuo não existe dificuldade nenhuma em encontrar os autovalores e autofunções pois sabemos que estes são as diferenças de massas quadradas e autoestados de massa no vácuo. Desta forma podemos escrever as probabilidades de oscilação para todos os sabores:

$$
\begin{aligned}
P_{\alpha \beta}(x) & =\left|\sum_{j=1}^{N} U_{\alpha j}^{*} e^{-i \frac{\Delta_{j 1}}{4 E}\left(x-x_{1}\right)} U_{\beta j}\right|^{2} \\
& =\delta_{\alpha \beta}-4 \sum_{(j k)}^{c i c l o} \operatorname{Re} J_{\alpha \beta}^{j k} \sin ^{2} \Delta_{j k} \pm 2 \sum_{(j k)}^{c i c l o} J \sin 2 \Delta_{j k}
\end{aligned}
$$

onde

$$
\begin{gathered}
J_{\alpha \beta}^{j k} \equiv U_{\alpha j} U_{\beta j}^{*}\left(U_{\alpha k} U_{\beta k}^{*}\right)^{*}, \quad J \equiv \operatorname{Im} J_{e \mu}^{12}, \\
\Delta_{j k} \equiv \frac{\Delta m_{j k}^{2}}{4 E}\left(x-x_{1}\right) .
\end{gathered}
$$


O sinal \pm no terceiro termo vale $-(+)$ no caso em que $(\alpha, \beta)$ seja dado pela permutação (anti) cíclica de $(e, \mu)$. A soma cíclica é sobre $(j k)=(12),(23),(31)$.

\subsubsection{Caso exponencial generalizado}

Em três gerações devemos encontrar seis raízes de dois polinômios de grau três ${ }^{3}$ : $\tilde{\mu}_{1,2,3}^{a}$ e $\tilde{\mu}_{1,2,3}^{b}$, para transformar a equação

$$
\begin{gathered}
{\left[\prod_{j=1}^{3}\left(i \frac{d}{d u}-\mu_{j}\right)-x_{0} V \frac{a_{1}}{a_{2}} \prod_{k=2}^{3}\left(i \frac{d}{d u}-\omega_{k}\right)\right] \psi+} \\
\frac{b_{2}}{a_{2}} e^{-u}\left[\prod_{j=1}^{3}\left(i \frac{d}{d u}-\mu_{j}\right)-x_{0} V \frac{b_{1}}{b_{2}} \prod_{k=2}^{3}\left(i \frac{d}{d u}-\omega_{k}\right)\right] \psi=0
\end{gathered}
$$

em

$$
\left[\prod_{j=1}^{3}\left(z \frac{d}{d z}-i \tilde{\mu}_{j}^{a}\right)+z \prod_{j=1}^{3}\left(z \frac{d}{d z}-i \tilde{\mu}_{j}^{b}\right)\right] \psi=0,
$$

onde já fizemos a mudança $z=-\frac{b_{2}}{a_{2}} e^{-u}$, cujas soluções são as funções hipergeométricas generalizadas

$$
\begin{aligned}
& \psi^{(1)}(z)=z^{i \tilde{\mu}_{1}^{a} F_{2}}\left[\begin{array}{ccc}
i \tilde{\mu}_{1}^{a}-i \tilde{\mu}_{1}^{b}, & i \tilde{\mu}_{1}^{a}-i \tilde{\mu}_{2}^{b}, & i \tilde{\mu}_{1}^{a}-i \tilde{\mu}_{3}^{b} \\
& 1+i \tilde{\mu}_{1}^{a}-i \tilde{\mu}_{2}^{a}, & 1+i \tilde{\mu}_{1}^{a}-i \tilde{\mu}_{3}^{a}
\end{array}\right] z \\
& \psi^{(2)}(z)=z^{i \tilde{\mu}_{2}^{a} F_{2}}\left[\begin{array}{ccc}
i \tilde{\mu}_{2}^{a}-i \tilde{\mu}_{1}^{b}, & i \tilde{\mu}_{2}^{a}-i \tilde{\mu}_{2}^{b}, & i \tilde{\mu}_{2}^{a}-i \tilde{\mu}_{3}^{b} \\
& 1+i \tilde{\mu}_{2}^{a}-i \tilde{\mu}_{1}^{a}, & 1+i \tilde{\mu}_{2}^{a}-i \tilde{\mu}_{3}^{a}
\end{array}\right] z \\
& \psi^{(3)}(z)=z^{i \tilde{\mu}_{3}^{a}}{ }_{3} F_{2}\left[\begin{array}{ccc}
i \tilde{\mu}_{3}^{a}-i \tilde{\mu}_{1}^{b}, & i \tilde{\mu}_{3}^{a}-i \tilde{\mu}_{2}^{b}, & i \tilde{\mu}_{3}^{a}-i \tilde{\mu}_{3}^{b} \\
& 1+i \tilde{\mu}_{3}^{a}-i \tilde{\mu}_{1}^{a}, & 1+i \tilde{\mu}_{3}^{a}-i \tilde{\mu}_{2}^{a}
\end{array}\right] z
\end{aligned}
$$

\section{Potencial variando exponencialmente}

Na Ref. [204] é apresentada a solução para um potencial que varia exponencialmente: $V(x) \propto e^{-x}$. Assim como fizemos em duas gerações, devemos tomar o limite $b_{2} \rightarrow 0$ para achar a mesma solução. Analogamente ao caso anterior de dois sabores, quando ${ }_{2} F_{1} \rightarrow{ }_{1} F_{1}$, aqui teremos ${ }_{3} F_{2} \rightarrow{ }_{2} F_{2}$, que são justamente as soluções obtidas em [204].

Não acreditamos que seja necessário colocar aqui todos os detalhes do extenso cálculo necessário para verificar a equivalência de nossos parâmetros com os obtidos na Ref. [204], cujas expressões são dadas também em função dos autovalores de massa na matéria.

\footnotetext{
${ }^{3} \mathrm{Na}$ verdade basta procurar as três raízes de um deles e fazer a troca $a_{1,2} \leftrightarrow b_{1,2}$.
} 


\subsubsection{Caso linear generalizado}

Novamente de modo sistemático, aplicamos o raciocínio empregado em 7.2.3. Aqui, como a solução obtida não possui artigo para comparação (apenas para o caso linear [197]), não faremos mudanças de variáveis.

Para abreviar a notação, denotamos

$$
\Pi_{\mu} \equiv \prod_{j=1}^{3}\left(i \frac{d}{d u}-\mu_{j}\right) \quad \text { e } \quad \Pi_{\omega} \equiv \prod_{k=2}^{3}\left(i \frac{d}{d u}-\omega_{k}\right)
$$

e analogamente $\Pi_{\tilde{\mu}_{a, b}}$ com os parâmetros $\tilde{\mu}_{1,2,3}^{a, b}$ definidos na seção anterior.

A transformada de Fourier da Eq. (7.51) faz com que

$$
\Pi_{\tilde{\mu}_{a, b}} \rightarrow P_{\tilde{\mu}_{a, b}}(t) \equiv \prod_{j=1}^{3}\left(t-\tilde{\mu}_{j}^{a, b}\right) \text { e } \Pi_{\omega} \rightarrow P_{\omega}(t) \equiv \prod_{k=2}^{3}\left(t-\omega_{k}\right)
$$

Desta forma, a expressão para a função de onda do neutrino eletrônico é

$$
\nu_{e}(u)=\int d t \frac{P_{\omega}(t)}{P_{\tilde{\mu}_{b}}(t)} e^{-i \frac{a_{2}}{b_{2}} \int^{t} d s P_{\tilde{\mu}_{a}}(s) / P_{\tilde{\mu_{b}}}(s)},
$$

cujas diferentes soluções linearmente independentes são encontradas limitando a integral pelas singularidades que são raízes de $P_{\tilde{\mu}_{b}}(t)$, ou seja, $\tilde{\mu}_{b}$.

\section{Potencial linear}

Quando o potencial tem a forma linear $V(x) \propto x$, devemos tomar o limite $b_{2} \rightarrow 0$. Entretanto é mais simples refazer as contas com $b_{2}=0$, como foi feito no caso de duas gerações.

Por este procedimento encontramos a expressão analítica apresentada em [197]

$$
\nu_{e}^{(n)}(u)=\int_{\omega_{n}}^{\omega_{n+1}} d \zeta e^{-i \zeta u} e^{-i \zeta^{2} / 2}\left(\prod_{j=2}^{3}\left|\zeta-\omega_{j}\right|^{i\left|\chi_{j}\right|^{2}}\right),
$$

onde $n=1,2,3, \omega_{1}=-\infty$ e $\omega_{4}=\infty$.

\subsection{Outros métodos - potencial constante}

A resolução de uma equação linear à coeficientes constantes é conhecida pela exponenciação de uma matriz. Entretanto, a expressão analítica se torna bem complicada para um problema em três dimensões, como vimos na seção anterior.

Na busca pela expressão completa, o caminho sistemático é fazer as contas 
longas sem se ater às sutilezas do problema em questão. Este é o método empregado por Zaglauer e Schwarzer em [209], que foram os primeiros a apresentarem um estudo completo das soluções exatas da equação de evolução com um potencial constante. Eles encontraram os autovalores de massas e os ângulos efetivos na matéria e então substituíram nas fórmulas de probabilidade de oscilação no vácuo. Uma década mais tarde Snellman e Ohlsson [210] propuseram um outro método, baseado no teorema de Cayley-Hamilton, para encontrar expressões mais simples para as probabilidades de oscilação em três gerações. E três anos depois, Kimura, Takamura e Yokomakura [211] propuseram outra forma de simplificar o cálculo direto das probabilidades de oscilação que foi generalizado para $N$ dimensões por Yasuda em 2007 [212].

\subsubsection{Autovalores efetivos na matéria}

Um estudo completo dos ângulos efetivos de mistura e autoestados de massa na matéria foi apresentado por Zaglauer e Schwarzer em 1988 [209]. Ainda que várias interpretações e explicações foram dadas para os resultados obtidos por Zaglauer e Schwarzer, o único método utilizado para determinação dos autovalores foi através da fórmula de Cardan para o polinômio característico de uma matriz $3 \times 3$. Acreditamos que seu resultado é o mais esclarecedor para os autoestados de massa na matéria e por isso o apresentamos aqui.

Denotando por $D=2 E V$ o potencial de matéria, o polinômio característico da hamiltoniana é

$$
\begin{gathered}
X^{3}-\left(m_{1}^{2}+m_{2}^{2}+m_{3}^{2}+D\right) X^{2}+\left[m_{1}^{2} m_{2}^{2}+m_{2}^{2} m_{3}^{2}+m_{1}^{2} m_{3}^{2}\right. \\
+D\left(m_{3}^{2} c_{13}^{2}+m_{2}^{2}\left(c_{13}^{2} c_{12}^{2}+s_{13}^{2}\right)+m_{1}^{2}\left(c_{13}^{2} s_{12}^{2}+s_{13}^{2}\right)\right] X \\
-\left[m_{1}^{2} m_{2}^{2} m_{3}^{2}+D\left(m_{3}^{2} m_{2}^{2} c_{13}^{2} c_{12}^{2}+m_{3}^{2} m_{1}^{2} c_{13}^{2} s_{12}^{2}+m_{2}^{2} m_{1}^{2} s_{13}^{2}\right)\right]=0
\end{gathered}
$$

o que leva aos autovalores de massa na matéria

$$
\begin{aligned}
& \tilde{m}_{1}^{2}=m_{1}^{2}+\frac{A}{3}-\frac{1}{3} \sqrt{A^{2}-3 B} S-\frac{\sqrt{3}}{3} \sqrt{A^{2}-3 B} \sqrt{1-S^{2}} \\
& \tilde{m}_{2}^{2}=m_{1}^{2}+\frac{A}{3}-\frac{1}{3} \sqrt{A^{2}-3 B} S+\frac{\sqrt{3}}{3} \sqrt{A^{2}-3 B} \sqrt{1-S^{2}} \\
& \tilde{m}_{3}^{2}=m_{1}^{2}+\frac{A}{3}+\frac{2}{3} \sqrt{A^{2}-3 B} S
\end{aligned}
$$


onde introduzimos, por conveniência, as abreviações

$$
\begin{gathered}
A=\Delta m_{21}^{2}+\Delta m_{31}^{2}+D \\
B=\Delta m_{31}^{2} \Delta m_{21}^{2}+D\left[\Delta m_{31}^{2} c_{13}^{2}+\Delta m_{21}^{2}\left(c_{13}^{2} c_{12}^{2}+s_{13}^{2}\right)\right] \\
C=D \Delta m_{31}^{2} \Delta m_{21}^{2} c_{13}^{2} c_{12}^{2} \\
S=\cos \frac{1}{3} \cos ^{-1} \frac{2 A^{3}-9 A B+27 C}{2 \sqrt[3]{A^{2}-3 B}}
\end{gathered}
$$

As diferenças dos autovalores que determinam os comprimentos de oscilação são dados por

$$
\begin{gathered}
\Delta \tilde{m}_{21}^{2}=\frac{2 \sqrt{3}}{3} \sqrt{A^{2}-3 B} \sqrt{1-S^{2}} \\
\Delta \tilde{m}_{31}^{2}=\sqrt{A^{2}-3 B} S+\frac{\sqrt{3}}{3} \sqrt{A^{2}-3 B} \sqrt{1-S^{2}} \\
\Delta \tilde{m}_{32}^{2}=\sqrt{A^{2}-3 B} S-\frac{\sqrt{3}}{3} \sqrt{A^{2}-3 B} \sqrt{1-S^{2}}
\end{gathered}
$$

de onde pode-se comprovar que eles só dependem dos valores de $\Delta m_{21}^{2}, \Delta m_{31}^{2}$, $\theta_{12}$ e $\theta_{13}$ devido à simetria no ângulo $\theta_{23}$ e fase $\delta$ apresentada na última seção do capítulo anterior.

\subsubsection{Teorema de Cayley-Hamilton}

O teorema de Cayley-Hamilton diz que o polinômio característico de uma matriz $M$ é satisfeito pela própria matriz $M$. Desta forma, supondo uma matriz $N \times N$, tem-se a seguinte igualdade $M^{N}=-c_{N-1} M^{N-1}-\cdots-c_{1} M-c_{0} I$. Utilizando esta fórmula recursivamente, a exponencial de uma matriz pode ser escrita como $e^{M}=a_{0} I+a_{1} M+\cdots+a_{N-1} M^{N-1}$. Aplicando esta idéia ao problema de neutrinos em três gerações Snellman e Ohlsson obtiveram uma expressão simplificada para o operador de evolução [210].

Ao invés de utilizar a matriz da hamiltoniana com potencial constante tradicional para a equação de evolução, eles trabalham na base de neutrinos autovetores do vácuo e subtraem um termo proporcional à identidade que torna a hamiltoniana de traço nulo. Isto vai simplificar algumas contas, pois denotando esta nova hamiltoniana por $T$, sabemos que o operador de evolução nesta base poderá ser escrito como $e^{-i x T}=a_{0} I-a_{1} i x T-a_{2} x^{2} T^{2}$. Para encontrar os coeficientes $a_{0}, a_{1}$ e $a_{2}$, basta escrever a identidade na base dos autovetores de $T$, ou seja, $e=\Lambda a \mathrm{com}$

$$
e=\left(\begin{array}{c}
e^{-i x \lambda_{1}} \\
e^{-i x \lambda_{2}} \\
e^{-i x \lambda_{3}}
\end{array}\right), \quad \Lambda=\left(\begin{array}{ccc}
1 & -i x \lambda_{1} & -x^{2} \lambda_{1}^{2} \\
1 & -i x \lambda_{2} & -x^{2} \lambda_{2}^{2} \\
1 & -i x \lambda_{3} & -x^{2} \lambda_{3}^{2}
\end{array}\right), \quad \text { e } \quad a=\left(\begin{array}{c}
a_{1} \\
a_{2} \\
a_{3}
\end{array}\right)
$$


onde $\lambda_{1}, \lambda_{2}$ e $\lambda_{3}$ são os autovalores de $T$, soluções do polinômio característico

$$
\lambda^{3}+c_{2} \lambda^{2}+c_{1} \lambda_{1}+c_{0}=0
$$

com $c_{2}=-\operatorname{Tr}(T)=0, c_{1}=\operatorname{det} T \operatorname{Tr}\left(T^{-1}\right)$ e $c_{0}=-\operatorname{det} T$. Quando estes sistemas são resolvidos, obtemos

$$
e^{-i x T}=\sum_{k=1}^{3} e^{-i x \lambda_{k}} \frac{1}{3 \lambda_{k}^{2}+c_{1}}\left[\left(\lambda_{k}^{2}+c_{1}\right) I+\lambda_{k} T+T^{2}\right] .
$$

Voltamos agora à base de autoestados de sabor, onde temos

$$
P_{\alpha \beta}(x)=\left|\left(\sum_{k=1}^{3} e^{-i x \lambda_{k}} \frac{1}{3 \lambda_{k}^{2}+c_{1}}\left[\left(\lambda_{k}^{2}+c_{1}\right) I+\lambda_{k} \tilde{T}+\tilde{T}^{2}\right]\right)_{\beta \alpha}\right|^{2},
$$

$\operatorname{com} \tilde{T} \equiv U T U^{-1}$. Se impusermos $\delta=0$ e $\tilde{x}_{a b} \equiv\left(\lambda_{a}-\lambda_{b}\right) / 2$, podemos chegar às seguintes fórmulas de probabilidades de transição (que incluem as duas independentes) para $\alpha \neq \beta$ e $\alpha, \beta=e, \mu, \tau$ :

$$
P_{\alpha \beta}(x)=-4 \sum_{a=1}^{3} \sum_{\substack{b=1 \\ a<b}}^{3} \frac{\lambda_{a} \tilde{T}_{\alpha \beta}+\left(\tilde{T}^{2}\right)_{\alpha \beta}}{3 \lambda_{a}^{2}+c_{1}} \frac{\lambda_{b} \tilde{T}_{\alpha \beta}+\left(\tilde{T}^{2}\right)_{\alpha \beta}}{3 \lambda_{b}^{2}+c_{1}} \sin ^{2} \tilde{x}_{a b} x
$$

Este método ainda permite determinar a matriz de mistura e os ângulos efetivos na matéria. Um simples cálculo mostra que

$$
\tilde{U}_{\alpha k} \tilde{U}_{\beta k}=\frac{\left(\lambda_{k}^{2}+c_{1}\right) \delta_{\alpha \beta}+\lambda_{k} \tilde{T}_{\alpha \beta}+\left(\tilde{T}^{2}\right)_{\alpha \beta}}{3 \lambda_{k}^{2}+c_{1}}
$$

E com este resultado podemos expressar os ângulos de mistura da seguinte maneira

$$
\begin{gathered}
\tilde{\theta}_{23} \equiv \tan ^{-1} \frac{\tilde{U}_{\mu 1}}{\tilde{U}_{\tau 1}}=\tan ^{-1} \frac{\lambda_{3} \tilde{T}_{e \mu}+\left(\tilde{T}^{2}\right)_{e \mu}}{\lambda_{3} \tilde{T}_{e \mu}+\left(\tilde{T}^{2}\right)_{e \mu}} \\
\tilde{\theta}_{13} \equiv \sin ^{-1} \tilde{U}_{e 1}=\sin ^{-1} \sqrt{\frac{\lambda_{3}^{2}+c_{1}+\lambda_{3} \tilde{T}_{e e}+\left(\tilde{T}^{2}\right)_{e e}}{3 \lambda_{3}^{2}+c_{1}}} \\
\tilde{\theta}_{12} \equiv \tan ^{-1} \frac{\tilde{U}_{e 2}}{\tilde{U}_{e 3}}=\tan ^{-1} \frac{\lambda_{2} \tilde{T}_{e \alpha}+\left(\tilde{T}^{2}\right)_{e \alpha}}{\lambda_{3} \tilde{T}_{e \alpha}+\left(\tilde{T}^{2}\right)_{e \alpha}} \frac{3 \lambda_{3}^{2}+c_{1}}{3 \lambda_{2}^{2}+c_{1}}, \alpha=\mu, \tau .
\end{gathered}
$$

Quando os neutrinos atravessam um meio com uma série de perfis de densidade constante, de larguras $L_{1}, \ldots, L_{n}$, o operador de evolução total é simplesmente dado por

$$
S(L)=\prod_{j=1}^{n} e^{-i x T}
$$


onde $L=L_{1}+\ldots+L_{n}$. Isto ocorre por exemplo, como já foi mencionado, no interior da Terra.

\subsubsection{Método de Kimura-Takamura-Yokomakura}

Como foi feito na Ref. [209] comentada em 7.4.1, uma das abordagens para obter as expressões das probabilidades de oscilação é calcular $\tilde{U}_{\alpha j}$ diagonalizando diretamente a hamiltoniana e daí calcular os produtos desses elementos, $\tilde{J}_{\alpha \beta}^{j k}$, que aparecem nas fórmulas das Eqs. (7.65) e (7.66). Entretanto, as expressões para as probabilidades obtidas nesta abordagem são complicadas e não é fácil de extrair informação dos efeitos de matéria e da violação CP. Kimura, Takamura e Yokomakura notaram que somente os produtos $\tilde{U}_{\alpha j} \tilde{U}_{\beta j}^{*}$ aparecem na probabilidade da Eq. (7.65) [211]. Então eles propuseram um método mais simples de obter tais produtos que foi generalizado para $N$ gerações por Yasuda [212].

Para simplificar a notação, chamamos $X_{j}^{\alpha \beta} \equiv U_{\alpha j} U_{\beta j}^{*}$ e $\tilde{X}_{j}^{\alpha \beta} \equiv \tilde{U}_{\alpha j} \tilde{U}_{\beta j}^{*}$. Apresentamos aqui o procedimento para três gerações, cuja generalização é evidente.

Da condição de unitariedade da matriz $\tilde{U}$, nós temos

$$
\delta_{\alpha \beta}=\left[\tilde{U} \tilde{U}^{-1}\right]_{\alpha \beta}=\sum_{j=1}^{3} \tilde{U}_{\alpha j} \tilde{U}_{\beta j}^{*}=\sum_{j=1}^{3} \tilde{X}_{j}^{\alpha \beta}
$$

Outra igualdade que podemos encontrar é

$$
\left[U \frac{M^{2}}{2 E} U^{\dagger}+V(x)\right]_{\alpha \beta}=\left[\tilde{U} \frac{\tilde{M}^{2}}{2 E} \tilde{U}\right]_{\alpha \beta}=\sum_{j=1}^{3} \tilde{U}_{\alpha j} \tilde{m}_{j}^{2} \tilde{U}_{\beta j}^{*}=\sum_{j=1}^{3} \tilde{m}_{j}^{2} \tilde{X}_{j}^{\alpha \beta}
$$

Além disso, podemos elevar ao quadrado a hamiltoniana para conseguir um última identidade independente

$$
\left[\left(U \frac{M^{2}}{2 E} U^{\dagger}+V(x)\right)^{2}\right]=\sum_{j=1}^{3}\left(\tilde{m}_{j}^{2}\right)^{2} \tilde{X}_{j}^{\alpha \beta} .
$$

Combinando as Eqs. (7.91), (7.92) e (7.93) na forma matricial obtemos um sistema linear, onde aparece a matriz de Vandermonde:

$$
\left(\begin{array}{ccc}
1 & 1 & 1 \\
\tilde{m}_{1}^{2} & \tilde{m}_{2}^{2} & \tilde{m}_{3}^{2} \\
\tilde{m}_{1}^{4} & \tilde{m}_{2}^{4} & \tilde{m}_{3}^{4}
\end{array}\right)\left(\begin{array}{c}
\tilde{X}_{1}^{\alpha \beta} \\
\tilde{X}_{2}^{\alpha \beta} \\
\tilde{X}_{3}^{\alpha \beta}
\end{array}\right)=\left(\begin{array}{c}
\delta_{\alpha \beta} \\
{\left[U \frac{M^{2}}{2 E} U^{\dagger}+V(x)\right]_{\alpha \beta}} \\
{\left[\left(U \frac{M^{2}}{2 E} U^{\dagger}+V(x)\right)^{2}\right]_{\alpha \beta}}
\end{array}\right),
$$

cuja inversa é conhecida, e a partir daí basta substituir o valor de $\tilde{X}_{j}^{\alpha \beta}$ na fórmula da Eq. (7.65). 


\section{Capítulo 8}

\section{Soluções aproximadas da equação de evolução}

Em geral existem poucos fenômenos ou processos físicos que podem ser resolvidos exatamente, e então para os outros problemas é necessário utilizar métodos de aproximação.

Hoje em dia existem vários métodos de aproximação que são usados na Física. A teoria de perturbação é uma das técnicas mais utilizadas em sistemas quânticos, mas existem outros métodos como, por exemplo, o variacional.

Neste último capítulo nós apresentamos os principais métodos de aproximação quando trabalhamos com a equação de evolução de neutrinos para um potencial arbitrário. Na primeira seção discutimos as motivações para os métodos empregados, isso inclui a redução de três para duas gerações. Na seção seguinte apresentamos os métodos que serão utilizados na busca por soluções aproximadas para as probabilidades de transição. A aproximação mais discutida no capítulo é a adiabática pelo seu vasto domínio de aplicação e sobretudo por sua simplicidade. A outra aproximação apresentada foi escolhida devida a sua simplicidade e por tratar de toda a região fora da ressonância, o que cobre boa parte das situações físicas. Na última parte fazemos um comentário sobre as aproximações feitas quando queremos tratar de problemas em três gerações.

\subsection{Motivações}

No capítulo anterior cobrimos todas as densidades de matéria que possuem soluções analíticas exatas. Como foi visto não se trata de uma lista grande e de fato muitos dos principais potenciais conhecidos na natureza não fazem parte da lista. Desta forma partimos então em busca de soluções aproximadas. Neste caso não precisamos especificar a forma do potencial, que então assumimos como 
arbitrário.

Abaixo segue uma breve discussão sobre a necessidade de fórmulas analíticas mais simples do que as encontradas para as soluções exatas. Após isso, apresentamos uma demonstração matemática de que devido aos valores obtidos experimentalmente para as diferenças de massas quadradas e ângulos de mistura podemos reduzir o problema inicialmente em três gerações para apenas duas.

\subsubsection{Complexidade das fórmulas exatas}

Com excessão da propagação no vácuo ou em um meio de densidade constante, todas as soluções encontradas estão apresentadas sob o sinal de integração (no caso linear generalizado) ou são dadas pelas chamadas funções especiais [207].

Em ambos os casos é difícil extrair significado físico das soluções. Se não fizéssemos uma análise para o caso constante, dificilmente veríamos que existe uma região de ressonância que amplifica a probabilidade de transição. A aplicação de métodos de aproximação visa, entre outras coisas, clarificar a física por trás de uma solução.

Além destes motivos, é evidente que a aplicação numérica de funções especiais não é tão simples. Elas possuem expressões analíticas em forma de séries, ou ainda podem ser dadas por uma representação integral. Isto tudo torna o processo de aplicação numérica mais lento. Sendo assim, um outro objetivo da procura por soluções aproximadas é encontrar soluções simples o suficiente para tornar tais cálculos mais rápidos.

\subsubsection{Redução de 3 gerações para 2 gerações}

Os resultados experimentais indicam que podemos fazer as seguintes aproximações:

$$
\begin{aligned}
\theta_{13} \simeq 0 & \Rightarrow \theta_{13} \rightarrow 0 . \\
\frac{\Delta m_{21}^{2}}{\Delta m_{31}^{2}} \ll 1 & \Rightarrow \frac{\Delta m_{21}^{2}}{\Delta m_{31}^{2}} \rightarrow 0
\end{aligned}
$$

Consideramos a equação de evolução dada por

$$
i \frac{d}{d x}|\nu\rangle=\left[\frac{1}{2 E} U \operatorname{diag}\left(0, \Delta m_{21}^{2}, \Delta m_{31}^{2}\right) U^{\dagger}+V(x) \operatorname{diag}(1,0,0)\right]|\nu\rangle,
$$

com $U=O_{23} U_{\delta} O_{13} O_{12}$ discutida no Capítulo 6. É simples ver que

$$
\left[O_{23} U_{\delta}, \operatorname{diag}(1,0,0)\right]=0
$$


Isto implica que na mudança $\left|\nu^{\prime}\right\rangle=U_{\delta}^{\dagger} O_{23}^{T}|\nu\rangle$ obtemos a equação

$$
i \frac{d}{d x}\left|\nu^{\prime}\right\rangle=\left[\frac{1}{2 E} O_{13} O_{12} \operatorname{diag}\left(0, \Delta m_{21}^{2}, \Delta m_{31}^{2}\right) O_{12}^{T} O_{13}^{T}+V(x) \operatorname{diag}(1,0,0)\right]\left|\nu^{\prime}\right\rangle .
$$

Utilizando a aproximação da Eq. (8.1) na Eq. (8.5) obtemos

$$
i \frac{d}{d x}\left|\nu^{\prime}\right\rangle=\left[\frac{\Delta m_{21}^{2}}{2 E}\left(\begin{array}{ccc}
s_{12}^{2} & s_{12} c_{12} & 0 \\
s_{12} c_{12} & c_{12}^{2} & 0 \\
0 & 0 & \frac{\Delta m_{31}^{2}}{\Delta m_{21}^{2}}
\end{array}\right)+V(x) \operatorname{diag}(1,0,0)\right]\left|\nu^{\prime}\right\rangle
$$

pois $O_{13} \rightarrow I$. Pela Eq. (8.6) vemos que a terceira componente de $\left|\nu^{\prime}\right\rangle$ se desacopla do sistema e evolui sozinha. Isto reduz o problema à apenas duas gerações, como gostaríamos de demonstrar. Entretando vamos verificar que esta aproximação é conveniente para explicar a oscilação encontrada para os neutrinos solares.

De fato, a mudança $\left|\nu^{\prime}\right\rangle=U_{\delta}^{\dagger} O_{23}^{T}|\nu\rangle$ significa que

$$
\begin{aligned}
\left|\nu_{e}^{\prime}\right\rangle & =\left|\nu_{e}\right\rangle \\
\left|\nu_{\mu}^{\prime}\right\rangle & =c_{23}\left|\nu_{\mu}\right\rangle-s_{23}\left|\nu_{\tau}\right\rangle \\
\left|\nu_{\tau}^{\prime}\right\rangle & =e^{-i \delta}\left(s_{23}\left|\nu_{\mu}\right\rangle+c_{23}\left|\nu_{e}\right\rangle\right)
\end{aligned}
$$

ou seja, mantém a primeira componente inalterada e mostra que não precisamos considerar a fase de violação CP. A Eq. (8.6) para as duas primeiras componentes é a mesma que no caso de duas gerações. Admitindo $V=0$ para uma breve análise no vácuo, a probabilidade de transição deve ser

$$
P_{2} \equiv P\left(\nu_{e} \leftrightarrow \nu_{\mu} ; x\right)=\sin ^{2} 2 \theta_{12} \sin ^{2}\left(\frac{\Delta m_{21}^{2}}{4 E} x\right)
$$

Das Eqs. (8.7) e (8.8), as oscilações dos neutrinos eletrônicos são determinadas por

$$
P\left(\nu_{e} \leftrightarrow \nu_{\mu} ; x\right)=c_{23}^{2} P_{2} \quad \text { e } \quad P\left(\nu_{e} \leftrightarrow \nu_{\tau} ; x\right)=s_{23}^{2} P_{2}
$$

Sendo assim a probabilidade de sobrevivência medida nos detectores é

$$
P_{e e}=1-P_{e \mu}-P_{e \tau}=1-\sin ^{2} 2 \theta_{12} \sin ^{2}\left(\frac{\Delta m_{21}^{2}}{4 E} x\right)
$$

controlada apenas pelo ângulo $\theta_{12}$ e diferença quadrada de massa $\Delta m_{21}^{2}$, como foi utilizado no Capítulo 4 para KamLAND.

Na aproximação da Eq. (8.2) os cálculos são todos parecidos, basta ver que 
$\left[O_{12}, \operatorname{diag}(0,0,1)\right]=0$ para obter a equação

$$
i \frac{d}{d x}\left|\nu^{\prime}\right\rangle=\left[\frac{\Delta m_{31}^{2}}{2 E}\left(\begin{array}{ccc}
s_{13}^{2} & 0 & s_{13} c_{13} \\
0 & 0 & 0 \\
s_{13} c_{13} & 0 & c_{13}^{2}
\end{array}\right)+V(x) \operatorname{diag}(1,0,0)\right]\left|\nu^{\prime}\right\rangle
$$

cujas soluções no vácuo para neutrinos eletrônicos são

$$
P\left(\nu_{e} \leftrightarrow \nu_{\mu} ; x\right)=s_{23}^{2} \tilde{P}_{2} \quad \text { e } \quad P\left(\nu_{e} \leftrightarrow \nu_{\tau} ; x\right)=c_{23}^{2} \tilde{P}_{2}
$$

com $\tilde{P}_{2} \equiv P_{2}\left(\Delta m_{21}^{2} \rightarrow \Delta m_{31}^{2}, \theta_{12} \rightarrow \theta_{13}\right)$. Isto mostra que o canal $\nu_{\mu} \leftrightarrow \nu_{e}$ fica suprimido pelo ângulo $\theta_{13}$ e que a oscilação observada em neutrinos atmosféricos deve estar entre a segunda e terceira família. Pode-se mostrar que nesta aproximação os detectores observam

$$
P_{\mu \mu}(x)=1-\sin ^{2} 2 \theta_{23} \sin ^{2}\left(\frac{\Delta m_{31}^{2}}{4 E} x\right) .
$$

\subsection{Métodos de aproximação}

Os métodos de aproximação de soluções desenvolvidos nesta parte são aqueles utilizados durante o capítulo: expansão perturbativa do operador de evolução, que utilizaremos para encontrar solução aproximada para um potencial arbitrário fora da zona de ressonância; e aproximação adiabática, quando um potencial qualquer varia lentamente com a posição.

Além destes dois, uma última seção é consagrada à uma breve apresentação de outros métodos que já foram utilizados na literatura e suas aplicações são comentadas.

\subsubsection{Expansão perturbativa do operador de evolução}

Consideremos o operador de evolução $S\left(x, x_{0}\right)$ definido em 6.4.1 para o caso de três gerações. Lembramos que este operador satisfaz a equação de evolução dos neutrinos

$$
i \frac{d}{d x} S\left(x, x_{0}\right)=H(x) S\left(x, x_{0}\right)
$$

com a condição inicial $S\left(x_{0}, x_{0}\right)=I$.

Agora decompomos a hamiltoniana em duas partes como $H(x)=H_{E}(x)+H_{I}$, onde $H_{E}(x)$ é de ordem zero em algum parâmetro de expansão e $H_{I}$ inclui os demais termos. Desta forma, o operador de evolução pode ser escrito como 
$S\left(x, x_{0}\right)=S_{E}\left(x, x_{0}\right) S_{I}\left(x, x_{0}\right)$, onde $S_{E}\left(x, x_{0}\right)$ satisfaz

$$
i \frac{d}{d x} S_{E}\left(x, x_{0}\right)=H_{E}(x) S_{E}\left(x, x_{0}\right), \quad S_{E}\left(x_{0}, x_{0}\right)=I ;,
$$

e assim a matriz $S_{I}\left(x, x_{0}\right)$ satisfaz

$$
i \frac{d}{d x} S_{I}\left(x, x_{0}\right)=S_{E}\left(x, x_{0}\right)^{-1} H_{I} S_{E}\left(x, x_{0}\right), \quad S_{I}\left(x_{0}, x_{0}\right)=I .
$$

É fácil ver que uma boa escolha de $H_{E}(x)$ é aquela em que podemos encontrar $S_{E}\left(x, x_{0}\right)$ exatamente.

Até agora nenhuma aproximação foi feita. Agora vamos encontrar o operador de evolução perturbativamente. Sendo $H_{1}$ a parte de $H_{I}$ na primeira ordem do parâmetro de perturbação escolhido, então desenvolvendo o operador de ordenamento temporal encontramos

$$
S\left(x, x_{0}\right) \simeq S_{E}\left(x, x_{0}\right)\left[I-i \int_{x_{0}}^{x}\left[S_{E}\left(s, x_{0}\right)^{-1} H_{1} S_{E}\left(s, x_{0}\right)\right] d s\right] .
$$

Este processo não depende do número de famílias e continua válido tanto em duas quanto em três gerações. Além disso, podemos prolongar a expansão do mesmo modo usando mais ordens do parâmetro de expansão escolhido para atingir a precisão desejada.

Neste método de aproximação os passos mais importantes são a escolha do parâmetro de expansão e a forma de divisão da hamiltoniana em $H_{E}(x)$ e $H_{I}$.

As probabilidades de oscilação são dadas por

$$
P_{\alpha \beta}(x)=\left|S_{\beta \alpha}\left(x, x_{0}\right)\right|^{2}
$$

$\operatorname{com} \alpha, \beta=e, \mu, \tau$.

\subsubsection{Aproximação adiabática}

Motivados pela seção anterior, só iremos trabalhar aqui com dois sabores de neutrinos. Além disso, o resultado em três gerações é tecnicamente mais complicado, porém utiliza a mesma idéia.

Na mudança de base dos neutrinos de sabores para os neutrinos autoestados instantâneos de massa, $|\tilde{\nu}\rangle=\tilde{U}(\tilde{\theta})|\nu\rangle$ encontramos a seguinte equação de evolução:

$$
i \frac{d}{d x}|\tilde{\nu}\rangle=\left[-\frac{\pi}{\tilde{\lambda}} \sigma_{3}+\frac{d}{d x} \tilde{\theta} \sigma_{2}\right]|\tilde{\nu}\rangle,
$$

onde $\sigma_{2}$ e $\sigma_{3}$ são a segunda e terceira matriz de Pauli e nós utilizamos o compri- 
mento de oscilação na matéria $\tilde{\lambda}=\frac{4 \pi E}{\Delta m^{2} C(x)}, C(x)=\sqrt{(A(x)-\cos 2 \theta)^{2}+\sin ^{2} 2 \theta}$ e $A(x)=\frac{2 E V(x)}{\Delta m^{2}}$. Pela definição do ângulo efetivo, $\sin 2 \tilde{\theta}=\frac{\sin 2 \theta}{C(x)}$, temos

$$
\frac{d}{d x} \tilde{\theta}=\frac{\Delta m^{2} \sin 2 \theta}{[4 E \Delta C(x)]^{2}} \frac{\dot{A}}{2}
$$

Para poder desprezar os termos fora da diagonal (que misturam os autovetores), devemos ter a seguinte condição

$$
\left|\frac{d}{d x} \tilde{\theta}\right| \ll \frac{\pi}{\tilde{\lambda}}
$$

O ponto em que a variação do ângulo é máxima deve ocorrer na posição de ressonância (isso não ocorre sempre). Desta forma introduzimos o parâmetro de adiabaticidade definido por

$$
\gamma=\frac{\frac{\pi}{\grave{\lambda}}}{\left|\frac{d}{d x} \tilde{\theta}\right|_{R}}=\frac{\Delta m^{2} \sin ^{2} 2 \theta}{2 E \cos 2 \theta\left|\frac{d}{d x} \ln A\right|_{R}},
$$

onde a derivada é calculada no ponto de ressonância. Com isto concluímos que se $\gamma \gg 1$ a evolução é adiabática, ou seja, a densidade da matéria varia lentamente e os autoestados de massa instântaneos não se misturam, e caso contrário, se $\gamma \sim 1$ devemos considerar esses efeitos de mistura.

\subsubsection{Outros métodos com expansão em série}

Obviamente existem outros métodos de encontrar soluções aproximadas com alguma expansão. Nesta parte comentamos a teoria de perturbação utilizada em sistemas quânticos para equações independentes do tempo e a resolução de equações diferenciais pela expansão em série.

\section{Teoria de perturbação}

A teoria de perturbação independente do tempo é descrita em praticamente todos os livros de MQ. Portanto não iremos desenvolvê-la aqui. Deixamos claro que ela possui importância apenas no caso de potencial constante, e como em duas gerações a solução exata é simples, só tem utilidade no caso de três famílias.

A aplicação deste método pode ser encontrada em [196], onde foram considerados os parâmetros de expansão $\alpha=\Delta m_{21}^{2} / \Delta m_{31}^{2}$ e $s_{13}=\sin \theta_{13}$. As soluções obtidas são expansões até primeira ordem em $\alpha$ ou em $s_{13}$, ou ainda até segunda ordem em $\alpha$ e $s_{13}$. 


\section{Solução com expansão em série}

A procura por soluções em série de equações diferenciais é um método muito utilizado em matemática.

Um desses métodos é o chamado método de Frobenius que resulta em séries de Laurent [207]. Este método é prático quando temos de resolver uma equação diferencial linear com coeficientes polinomiais. Isto pode ser obtido através da expansão do potencial em série de Taylor.

A Ref. [213] apresenta um estudo da solução em série para um potencial qualquer. Ele não apresenta um resultado simples, sobretudo para a fórmula de recorrência dos coeficientes. Nós tentamos aplicar o método para potenciais simples, mas também não obtivemos resultado significativo, ou seja, simples de usar e compreensíveis. As soluções obtidas por este método devem ser truncadas em algum grau da série, o que pode torná-la muito longe da solução real dependendo das condições do problema.

\subsection{Aproximação adiabática}

Nesta seção descrevemos os resultados existentes quando utilizamos a aproximação adiabática na resolução da equação de evolução. Além disso, quando os neutrinos passam por uma região de não adiabaticidade, $\gamma \sim 1$, mas só são detectados muito tempo depois, existem fórmulas que corrigem as probabilidades de oscilação obtidas na aproximação adiabática e consideram a amplitude de transição entre os autoestados de massa instântaneos. Na última parte desta seção, lidamos com o tratamento simples utilizado na aproximação adiabática no caso de três gerações.

\subsubsection{Solução adiabática}

Considerando um feixe inicial de neutrinos eletrônicos numa posição $x_{i}$ em que o ângulo de mistura efetivo é dado por $\tilde{\theta}\left(x_{i}\right)=\theta_{i}$, o feixe inicial é descrito na base dos autoestados de massa instantâneos, por

$$
\left|\nu\left(x_{i}\right)\right\rangle=\left|\nu_{e}\right\rangle=\cos \theta_{i}\left|\tilde{\nu}_{1}\right\rangle+\sin \theta_{i}\left|\tilde{\nu}_{2}\right\rangle
$$

Então, na aproximação adiabática, numa posição $x_{f}$ teremos

$$
\left|\nu\left(x_{f}\right)\right\rangle=\cos \theta_{i}\left|\tilde{\nu}_{1}\right\rangle+\sin \theta_{i} e^{-i \int_{x_{i}}^{x_{f}} d x \frac{\Delta m^{2} C(x)}{2 E}}\left|\tilde{\nu}_{2}\right\rangle
$$

Levando em consideração que na posição final o ângulo de mistura efetivo é 
diferente daquele na posição inicial temos

$$
\left|\nu_{Y}\right\rangle=-\sin \theta_{f}\left|\tilde{\nu}_{1}\right\rangle+\cos \theta_{f}\left|\tilde{\nu}_{2}\right\rangle
$$

e portanto

$$
P_{2}\left(\nu_{e} \rightarrow \nu_{Y} ; x_{f}\right)=\frac{1}{2}-\frac{1}{2} \cos 2 \theta_{i} \cos 2 \theta_{f}-\frac{1}{2} \sin 2 \theta_{i} \sin 2 \theta_{f} \cos \phi\left(x_{i}, x_{f}\right),
$$

onde encontramos uma fase, denominada fase adiabática, dada por

$$
\phi\left(x_{i}, x_{f}\right) \equiv \frac{\Delta m^{2}}{2 E} \int_{x_{i}}^{x_{f}} d x C(x)=\frac{\Delta m^{2}}{2 E} \int_{x_{i}}^{x_{f}} d x \sqrt{(A(x)-\cos 2 \theta)^{2}+\sin ^{2} 2 \theta} .
$$

Nota-se que a solução da Eq. (8.25) é simétrica na troca da posição inicial com a final $x_{i} \leftrightarrow x_{f}$. Além disso, se a densidade de matéria no ponto de produção do neutrino estiver muito acima do ponto de ressonância MSW, então $\sin 2 \theta_{i} \simeq 0$ e o terceiro termo da Eq. (8.25) fica extremamente suprimido.

\subsubsection{Feixe de neutrinos com distribuição energética}

Se considerarmos um feixe com distribuição energética, após uma longa trajetória o termo oscilante é mediado à zero. Dizemos que o feixe deixa de ser coerente. Sendo assim, para simplificar os cálculos, podemos trabalhar com a solução descoerente, cuja fórmula na aproximação adiabática é

$$
P_{2}\left(\nu_{e} \rightarrow \nu_{Y} ; x_{f}\right)=\frac{1}{2}\left[1-\cos 2 \theta_{i} \cos 2 \theta_{f}\right]
$$

Quando trabalhamos com feixes descoerentes, não é necessário fazer todo o cálculo exato de neutrinos monocromáticos para se chegar à expressão analítica da probabilidade de oscilação. Neste caso, podemos fazer a soma incoerente sobre os autoestados de massa durante a propagação:

$$
\begin{aligned}
P_{e e} & =\sum_{j} P_{f}\left(\nu_{e} \rightarrow \nu_{j}\right) P_{i}\left(\nu_{j} \rightarrow \nu_{e}\right) \\
& =\left[\begin{array}{ll}
1 & 0
\end{array}\right]\left[\begin{array}{cc}
\cos ^{2} 2 \theta_{f} & \sin ^{2} 2 \theta_{f} \\
\sin ^{2} 2 \theta_{f} & \cos ^{2} 2 \theta_{f}
\end{array}\right]\left[\begin{array}{cc}
1 & 0 \\
0 & 1
\end{array}\right]\left[\begin{array}{cc}
\cos ^{2} 2 \theta_{i} & \sin ^{2} 2 \theta_{i} \\
\sin ^{2} 2 \theta_{i} & \cos ^{2} 2 \theta_{i}
\end{array}\right]\left[\begin{array}{l}
1 \\
0
\end{array}\right] . \\
& =\sin ^{2} \theta_{f} \sin ^{2} \theta_{i}+\cos ^{2} \theta_{f} \cos ^{2} \theta_{i}=\frac{1}{2}\left(1+\cos 2 \theta_{f} \cos 2 \theta_{i}\right)
\end{aligned}
$$

Vejamos agora o que esse resultado significa fisicamente. Assumimos que a densidade de matéria seja novamente decrescente durante a trajetória do neutrino. Consideramos também que neutrinos eletrônicos sejam produzidos numa região de alta densidade, bem acima da zona de ressonância MSW. Neste caso 
$\theta_{i} \simeq \pi / 2$, o que significa que a mistura de neutrinos está fortemente suprimida pelo efeito de matéria. Durante a propagação dos neutrinos à regiões de densidades menores, sua mistura aumenta e se torna máxima no ponto de ressonância. Conforme os neutrinos continuam à se propagar em direção de densidades menores, seu ângulo de mistura efetivo diminui até atingir o valor do ângulo no vácuo, $\theta_{f} \simeq \theta$, quando $A \ll A_{R}=\cos 2 \theta$. A Eq. (8.28) fornece então o seguinte valor de probabilidade de transição:

$$
P_{e \mu}=1-P_{e e} \simeq \cos ^{2} \theta
$$

ou seja, no caso de ângulo de mistura no vácuo pequeno, podemos ter conversão adiabática de $\nu_{e}$ para $\nu_{\mu}$ quase completa. Isto está ilustrado na Figura 8.1, que mostra os níveis de energia dos autoestados de matéria $\tilde{\nu}_{1}$ e $\tilde{\nu}_{2}$. No ponto de produção, onde $\theta_{i} \simeq \pi / 2$ o $\nu_{e}$ produzido quase coincide com o autoestado $\tilde{\nu}_{2}$. Se a densidade de matéria varia adiabaticamente durante a trajetória do neutrino, as transições $\tilde{\nu}_{1} \leftrightarrow \tilde{\nu}_{2}$ ficam exponencialmente suprimidas como veremos mais adiante. Assim um sistema produzido no autoestado $\tilde{\nu}_{2}$ permanecerá neste mesmo estado durante todo a trajetória. Entretanto, a composição dos sabores em função dos autoestados de matéria muda com a posição, e no ponto final de evolução o autoestado de matéria $\tilde{\nu}_{2}$ tem a componente do $\nu_{e}$ com peso de $\sin ^{2} \theta$ (e não mais $\sin ^{2} \theta_{i}$ ), e de $\nu_{\mu}$ com peso $\cos ^{2} \theta$, explicando assim o resultado obtido.

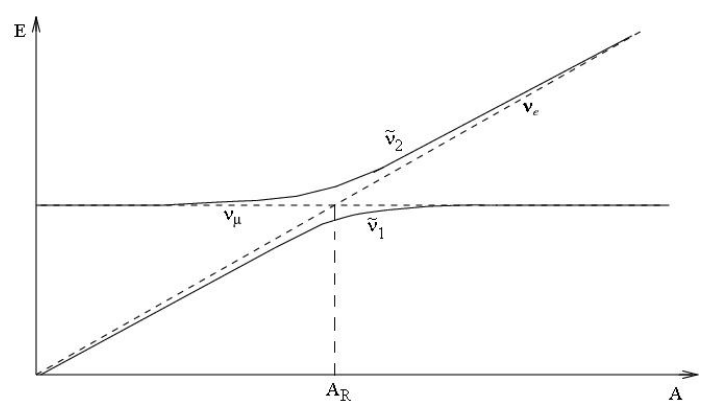

Figura 8.1: Níveis de energia na matéria por $A$. Na ausência de mistura - linhas tracejadas, com mistura - linhas sólidas.

\subsubsection{Correções à aproximação adiabática}

Analogamente ao efeito túnel que uma partícula pode sofrer, um estado de energia mais baixa pode transitar para um estado de energia mais alta caso exista algum instante (mesmo complexo, o que caracteriza a natureza quântica do fenômeno) em que estas duas energias são iguais.

Landau propôs um método para calcular esta probabilidade de transição 
através da evolução de um estado passando pelo ponto de igualdade das energias [162, 164, 163]. Vamos desenvolvê-lo aplicado ao contexto de neutrinos.

\section{Método de Landau}

Consideramos a Eq. (8.19), onde vemos que a diferença dos autovalores que é $\Delta \tilde{m}^{2}=\Delta m^{2} C(x)$ se anula quando $C(x)=0$, ou seja, $A\left(x_{c}\right)=e^{ \pm i 2 \theta}$. A probabilidade de transição de $\left|\tilde{\nu}_{1}\right\rangle \rightarrow\left|\tilde{\nu}_{2}\right\rangle$ se dá pela evolução que passa pelo ponto $x_{c}$. Como primeira aproximação, digamos que o neutrino seja preparado em $x_{i} \rightarrow-\infty$ no estado $\left|\tilde{\nu}_{1}\right\rangle$ e detectado em $x_{f} \rightarrow \infty$. Utilizando a analiticidade da hamiltoniana em questão, podemos escolher qualquer trajetória de integração no operador de evolução, contanto que ela passe por $x_{c}$ onde o estado troca de autovalor. O caminho mais simples é aquele que o neutrino caminha pelo eixo real durante toda a trajetória até $r_{c}=\operatorname{Re}\left[x_{c}\right]$, quando percorre pelo eixo imaginário até $x_{c}$ e retorna com energia diferente. Desta forma, desprezando a coerência, obtemos

$$
\begin{aligned}
|\nu(x \rightarrow \infty)\rangle= & e^{-i \int_{r_{c}}^{\infty} d s \tilde{m}_{2}^{2}(s) / 2 E} e^{-i \int_{x_{c}}^{r_{c}} d s \tilde{m}_{2}^{2}(s) / 2 E}\left|\tilde{\nu}_{2}\right\rangle \times \\
& \times\left\langle\tilde{\nu}_{1}\left|e^{-i \int_{r_{c}}^{x_{c}} d s \tilde{m}_{1}^{2}(s) / 2 E} e^{-i \int_{-\infty}^{r_{c}} d s \tilde{m}_{1}^{2}(s) / 2 E}\right| \tilde{\nu}_{1}\right\rangle,
\end{aligned}
$$

de onde encontramos a amplitude de transição

$$
\left\langle\tilde{\nu}_{2} \mid \nu(x \rightarrow \infty)\right\rangle=e^{-i \int_{r_{c}}^{\infty} d s \tilde{m}_{2}^{2}(s) / 2 E} e^{-i \int_{-\infty}^{r_{c}} d s \tilde{m}_{1}^{2}(s) / 2 E} e^{i \int_{r_{c}}^{x_{c}} d s \Delta \tilde{m}^{2}(s) / 2 E} .
$$

Note que apenas a última exponencial possui uma integral de caminho sobre o eixo complexo e por isso é o único termo que não é uma fase. Desta forma achamos a probabilidade de transição conhecida por probabilidade de LandauZener [165, 214]:

$$
P_{L Z}=\left|\left\langle\tilde{\nu}_{2} \mid \nu(x \rightarrow \infty)\right\rangle\right|^{2}=e^{-2 \frac{\Delta m^{2}}{2 E} \operatorname{Im}\left[\int_{r_{c}}^{x_{c}} d s \sqrt{\left((A(s)-\cos 2 \theta)^{2}+\sin ^{2} 2 \theta\right.}\right]} .
$$

Lembramos que existem dois pontos soluções de $A\left(x_{c}\right)=e^{ \pm i 2 \theta}$, a escolha deve ser feita tal que $P_{L Z}<1$, pois senão a solução não é fisicamente possível. Ressaltamos também que $P_{L Z}$ não é um resultado exato e sim obtido por um método aproximado e assintótico de cálculo.

O fato da fórmula de $P_{L Z}$ na Eq. (8.31) possuir uma integral relativamente complicada (com raiz quadrada), torna impossível de encontrar uma expressão analítica sem integração para um potencial qualquer. De fato, na Ref. [160] é apresentada uma extensa tabela com os métodos utilizadas na resolução desta integral para as formas mais simples de potencial. Contudo, não é difícil a 
obtenção de $P_{L Z}$ para os casos de potencial linear, exponencial e $1 / x$, onde encontramos respectivamente:

$$
P_{L Z}=e^{-\frac{\pi}{2} \gamma}, \quad P_{L Z}=e^{-\frac{\pi}{2} \gamma\left(1-\tan ^{2} \theta\right)} \text { e } \quad P_{L Z}=e^{-\frac{\pi}{2} \gamma\left(1-\tan ^{2} \theta\right) /\left(1+\tan ^{2} \theta\right)} .
$$

Desta forma introduzimos um parâmetro $F$ tal que toda probabilidade de transição calculada por este método seja dada por $P_{L Z}=e^{-\frac{\pi}{2} \gamma F}$.

\section{Aplicação nas fórmulas de probabilidades de oscilação}

Agora vamos introduzir esta probabilidade de transição no problema onde o feixe de neutrinos descoerente passe pela zona de ressonância. Neste caso basta trocar a matriz identidade por aquela com a probabilidade de transição:

$$
\begin{aligned}
P_{e e}= & {\left[\begin{array}{ll}
1 & 0
\end{array}\right]\left[\begin{array}{cc}
\cos ^{2} 2 \theta_{f} & \sin ^{2} 2 \theta_{f} \\
\sin ^{2} 2 \theta_{f} & \cos ^{2} 2 \theta_{f}
\end{array}\right]\left[\begin{array}{cc}
1-P_{L Z} & P_{L Z} \\
P_{L Z} & 1-P_{L Z}
\end{array}\right] \times } \\
& {\left[\begin{array}{cc}
\cos ^{2} 2 \theta_{i} & \sin ^{2} 2 \theta_{i} \\
\sin ^{2} 2 \theta_{i} & \cos ^{2} 2 \theta_{i}
\end{array}\right]\left[\begin{array}{l}
1 \\
0
\end{array}\right] } \\
= & \frac{1}{2}+\left(\frac{1}{2}-P_{L Z}\right) \cos 2 \theta_{f} \cos 2 \theta_{i}
\end{aligned}
$$

No exemplo mencionado em 8.3.2 não consideramos a probabilidade $P_{L Z}$ de troca $\tilde{\nu}_{1} \leftrightarrow \tilde{\nu}_{2}$. A inclusão deste valor deve diminuir a probabilidade de conversão adiabática $P_{e \mu}$. De fato, substituindo os valores do exemplo na Eq. (8.32) obtemos

$$
P_{e \mu} \simeq \cos ^{2} \theta-P_{L Z} \cos 2 \theta \simeq 1-P_{L Z}
$$

quando $\theta_{f} \simeq \theta \simeq 0$.

\section{Cálculo exato de $P_{L Z}$}

Existe, porém, um outro método de obter $P_{L Z}$ com as condições assintóticas e que deve fornecer um resultado mais preciso. A idéia é utilizar as soluções exatas apresentadas no capítulo anterior e observar o comportamento das funções nos limites extremos, e assim calcular $P_{e e}$ identificando $P_{L Z}$ pela Eq. (8.32).

Com auxílio da Eq. (7.58) obtemos $P_{L Z}=e^{-\frac{\pi}{2} \gamma}$ como calculado com o método de Landau. Contudo, se utilizamos a solução na Eq. (7.49) encontramos

$$
P_{L Z}=\frac{e^{-\frac{\pi}{2} \gamma F}-e^{-\frac{\pi}{2} \gamma\left(\frac{F}{\sin ^{2} \theta}\right)}}{1-e^{-\frac{\pi}{2} \gamma\left(\frac{F}{\sin ^{2} \theta}\right)}},
$$

com $F=1-\tan ^{2} \theta$ como calculado no método de Landau, porém a probabilidade de transição obtida é diferente. Isto ocorre de maneira semelhante com a solução 
para $V(x) \propto 1 / x[160]$. Na próxima seção discutimos o motivo dessa forma de $P_{L Z}$.

\subsubsection{Ansatz de Kuo e Pantaleone}

Em seu artigo Ref. [160], Kuo e Pantaleone analisam a razão da discrepância apontada acima. A maneira mais simples de ver é colocando um potencial do tipo escada onde $\dot{\tilde{\theta}}=\delta(x)$ e portanto $\gamma \rightarrow 0$, ou seja, onde se atinge o limite extremo de não adiabaticidade. Nesta situação temos

$$
\begin{aligned}
\operatorname{Amp}\left(\nu_{i}^{(1)} \rightarrow \nu_{j}^{(2)}\right) & =\left(\begin{array}{cc}
\cos \theta_{2} & -\sin \theta_{2} \\
\sin \theta_{2} & \cos \theta_{2}
\end{array}\right)\left(\begin{array}{cc}
\cos \theta_{1} & -\sin \theta_{1} \\
\sin \theta_{1} & \cos \theta_{1}
\end{array}\right) \\
& =\left(\begin{array}{cc}
\cos \left(\theta_{1}-\theta_{2}\right) & \sin \left(\theta_{1}-\theta_{2}\right) \\
-\sin \left(\theta_{1}-\theta_{2}\right) & \cos \left(\theta_{1}-\theta_{2}\right)
\end{array}\right)
\end{aligned}
$$

e assim $P_{L Z}=\sin ^{2}\left(\theta_{1}-\theta_{2}\right)$. Chamando os potenciais constantes referentes às posições de $\theta_{1}$ e $\theta_{2}$ de $A_{1}$ e $A_{2}$, respectivamente, vemos que se $A_{1} \rightarrow A_{2}$ (potencial constante) obtemos o limite extremo de adiabaticidade $(\gamma \rightarrow \infty)$ e $P_{L Z} \rightarrow 0$. Se $A_{1}$ está muito acima da zona de ressonância $\left(\theta_{1} \rightarrow \frac{\pi}{2}\right)$ e $A_{2} \rightarrow 0$ (vácuo), temos o limite extremo de não adiabaticidade $(\gamma \rightarrow 0)$, e $P_{L Z}=\cos ^{2} \theta$.

Quando calculamos $P_{L Z}$ apenas pelo método de Landau, sempre encontramos $P_{L Z} \rightarrow 0$ se $\gamma \rightarrow \infty$, entretanto para alguns potenciais que passam pelo limite extremo de não adiabaticidade $(\gamma \sim 1)$ devemos encontrar $P_{L Z} \rightarrow \cos ^{2} \theta$ quando $\gamma \rightarrow \infty$. Por isso, baseado nos dois resultados conhecidos $V(x) \propto 1 / x$ e $e^{-x}$, e na análise da solução para o caso $V(x) \propto 1+\tanh x$, Kuo e Pantaleone propõem a fórmula da Eq. (8.34) como um Ansatz para a correção de todos os potenciais que em algum ponto alcançam $\gamma \lesssim 1$.

\subsubsection{Aproximação adiabática e o efeito MSW}

A fórmula na Eq. (8.32) é a forma final que utilizamos no cálculo pela aproximação adiabática. Independemente do método utilizado para encontrar $P_{L Z}$, podemos usar tal solução para reencontrar o efeito MSW.

De fato, fazendo o gráfico de $P_{e e}$ cmo função de $4 E / \Delta m^{2}$ obtemos a Figura 8.2. Nesta figura, levando em consideração os efeitos de matéria (b) e (c), fica visível a região dentro da qual ocorre ampliação da probabilidade de conversão. esta é a zona de ressonância discutida anteriormente. Quanto menor o ângulo de mistura no vácuo, maior a supressão da probabilidade de sobrevivência $P_{e e}$. 


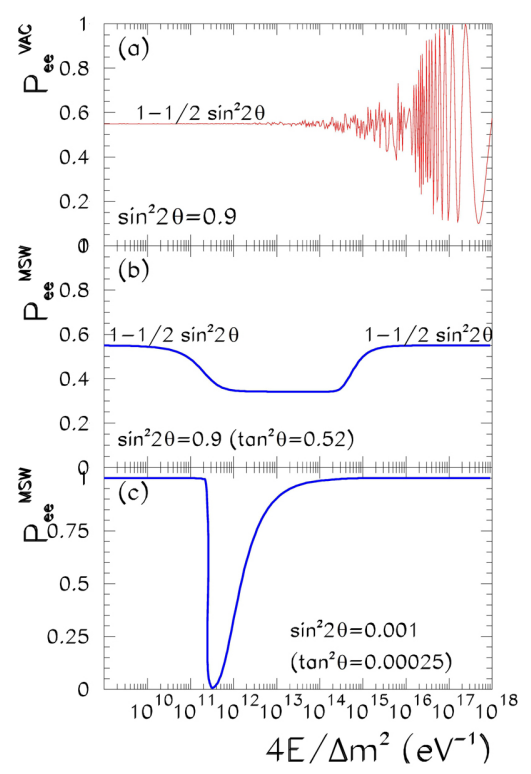

Figura 8.2: $P_{e e}$ como função de $4 E / \Delta m^{2}$. No quadro (a) os efeitos de matéria são ignorados. Nos painéis (b) (ângulo de mistura grande) e (c) (ângulo de mistura pequeno) o efeito MSW é levado em consideração.

\subsubsection{Aplicação em três gerações}

Se desenvolvermos a equação de evolução em três gerações na base dos autoestados de massa veremos que a aproximação adiabática neste caso consiste em ignorar o termo $\tilde{U}^{\dagger} \frac{d}{d x} \tilde{U}$, como no caso de duas gerações [159]. Mais precisamente, é equivalente à desprezar os termos $\left|\frac{d}{d x} \tilde{\theta}_{12}\right|,\left|\sin \tilde{\theta}_{12} \frac{d}{d x} \tilde{\theta}_{13}\right|$ e $\left|\cos \tilde{\theta}_{12} \frac{d}{d x} \tilde{\theta}_{13}\right|$ frente aos autovalores de massa efetivos.

A aproximação adiabática só recebe correções próximas às ressonâncias. No menor ponto em que ocorre ressonância, $\left|\frac{d}{d x} \tilde{\theta}_{12}\right|$ é grande e pode ocorrer transiçào entre as famílias 1 e 2. Na ressonância superior, $\left|\frac{d}{d x} \tilde{\theta}_{13}\right|$ é que é grande e pode ocorrer transição entre as famílias 2 e 3. Sendo assim, a fórmula de probabilidade de oscilação em três gerações desprezando a coerência na aproximação adiabática é

$$
\begin{aligned}
& P_{e e}=\left[\left|\tilde{U}_{e 1}^{f}\right|^{2}\left|\tilde{U}_{e 2}^{f}\right|^{2}\left|\tilde{U}_{e 3}^{f}\right|^{2}\right] X_{12} X_{23}\left[\begin{array}{c}
\left|\tilde{U}_{e 1}^{i}\right|^{2} \\
\left|\tilde{U}_{e 2}^{i}\right|^{2} \\
\left.\tilde{U}_{e 3}^{i}\right|^{2}
\end{array}\right], \\
& X_{12}=\left[\begin{array}{ccc}
1-P_{L Z} & P_{L Z} & 0 \\
P_{L Z} & 1-P_{L Z} & 0 \\
0 & 0 & 1
\end{array}\right] \quad \text { e } \quad X_{23}=\left[\begin{array}{ccc}
1 & 0 & 0 \\
0 & 1-P_{L Z}^{\prime} & P_{L Z}^{\prime} \\
0 & P_{L Z}^{\prime} & 1-P_{L Z}^{\prime}
\end{array}\right] \text {, }
\end{aligned}
$$

em que $P_{L Z}$ e $P_{L Z}^{\prime}$ se diferenciam pois são calculados em dois pontos distintos. 


\subsection{Aproximação fora da zona de ressonância}

$\mathrm{Na}$ análise dos resultados experimentais comentamos que apenas os neutrinos solares passavam pela região de ressonância. De fato, quando trabalhamos com neutrinos atmosféricos e de aceleradores, suas energias são muito elevadas e desta forma podemos utilizar uma outra aproximação. Neste caso desenvolvemos a expansão do operador de evolução com o ângulo efetivo na matéria como parâmetro de expansão, $\epsilon=\sin 2 \theta_{m}$, já que ele é pequeno no limite de altas energias [215]

$$
\frac{2 E V(x)}{\Delta m^{2}} \gg \cos 2 \theta
$$

ou seja, acima da zona de ressonância de MSW.

A divisão da hamiltoniana é feita da seguinte forma

$$
H_{E}(x)=\Delta C(x)\left(\begin{array}{cc}
1 & 0 \\
0 & -1
\end{array}\right) \quad \text { e } H_{I}=\sin 2 \theta \Delta\left(\begin{array}{cc}
\xi & 1 \\
1 & -\xi
\end{array}\right) \text {. }
$$

Como gostaríamos, a razão entre o segundo e o primeiro termos da hamiltoniana é determinado pelo ângulo de mistura na matéria $\epsilon=\sin 2 \theta_{m}=\frac{\sin 2 \theta}{C(x)}$. A diferença $\xi$ é

$$
\xi \equiv \frac{\cos 2 \theta \Delta-V / 2+\Delta C(x)}{\sin 2 \theta \Delta} \approx \frac{\Delta}{V} \sin 2 \theta \ll 1 .
$$

Então $\xi \sim \epsilon$, e assim os termos na diagonal de $H_{I}$ podem ser desprezados na aproximação de menor ordem.

Como $H_{E}(x)$ é diagonal temos

$$
S_{E}\left(x, x_{0}\right)=\left(\begin{array}{cc}
e^{-i \phi\left(x_{0}, x\right) / 2} & 0 \\
0 & e^{i \phi\left(x_{0}, x\right) / 2}
\end{array}\right)
$$

onde $\phi\left(x_{0}, x\right)$ é a fase adiabática definidana Eq. (8.26). Como $H_{1}$ é

$$
H_{1}=\sin 2 \theta \Delta\left(\begin{array}{ll}
0 & 1 \\
1 & 0
\end{array}\right)
$$

é fácil ver da Eq. (8.17) que

$$
S\left(x, x_{0}\right)=S_{E}\left(x, x_{0}\right)\left[I-i \Delta \sin 2 \theta \int_{x_{0}}^{x} d s\left(\begin{array}{cc}
0 & e^{i \phi\left(x_{0}, s\right)} \\
e^{-i \phi\left(x_{0}, s\right)} & 0
\end{array}\right)\right]
$$

Isto implica na seguinte probabilidade de transição

$$
P_{2}=\left|S_{Y e}\right|^{2}=\left(\frac{\Delta m^{2}}{4 E}\right)^{2} \sin ^{2} 2 \theta\left|\int_{x_{0}}^{x} d s e^{i \phi\left(x_{0}, s\right)}\right|^{2} .
$$


Para potenciais cujas densidades são simétricas em relação ao ponto no interior da trajetória dos neutrinos, como ocorre no interior da Terra $V(x)=$ $V\left(x_{\text {diam }}-x\right)$, a Eq. (8.44) se torna

$$
P_{2, \operatorname{sim}}=4\left(\frac{\Delta m^{2}}{4 E}\right)^{2} \sin ^{2} 2 \theta\left[\int_{x_{0}}^{x / 2} d z \cos \phi\left(x_{0}, z\right)\right]^{2}
$$

onde $z=s-x / 2$ é a distância até o centro da trajetória.

Um cálculo direto e extenso mostra que na próxima (segunda) ordem em $\epsilon$ somente os elementos diagonais de $S$ são modificados, enquanto as amplitudes de transição $S_{Y e}$ só são corrigidas a partir da terceira ordem $\epsilon^{3}: S_{Y e} \approx\left[O(\epsilon)+O\left(\epsilon^{3}\right)\right]$. Sendo assim, as correções para as probabilidades $P_{2}$ são da ordem $\epsilon^{4}$. Isto explica a notável precisão das fórmulas obtidas.

Outro ponto interessante desta aproximação é que o resultado permanece válido para baixas energias caso $\frac{2 E V(x)}{\Delta m^{2}} \ll \cos 2 \theta$ seja satisfeito. Neste caso apenas algumas modificações devem ser feitas: o sinal de $H_{E}(x)$ tem que ser trocado, pois ocorre a troca dos autoestados na passagem pela zona de ressonância; e assim dos autovalores $\Delta C(x) \rightarrow-\Delta C(x)$. Contudo, a expressão da probabilidade de transição permanece a mesma.

\subsection{Aproximações em três gerações}

A análise das probabilidades em duas gerações é bem mais simples do que em três. Isso também é válido na procura por soluções aproximadas, como já pode ser verificado pela aproximação adiabática, cujo resultado em três gerações seguiu imediatamente das contas em duas. Desta forma, a maneira tradicional de analisar os resultados em três gerações não é partindo da equação de evolução com três famílias, mas sim adicionando os parâmetros descartados na redução apresentada em 8.1.2 [68].

\section{Inclusão de $\theta_{13}$ na análise de neutrinos solares}

Para análise dos neutrinos solares, tomamos o limite $\theta_{13} \rightarrow 0$. Desta forma, a primeira tentativa para generalizar as contas em três gerações é incluir os efeitos deste ângulo nas fórmulas de probabilidade de oscilação.

A maneira mais simples que alguém pode pensar em fazer é utilizar o outro limite, $\Delta m_{21}^{2} / \Delta m_{31}^{2} \rightarrow 0$, em que sabemos que o ângulo que controla a oscilação é $\theta_{13}$. Contudo, como a Eq. (8.12) mostra, teríamos $P_{e e}=1-\tilde{P}_{2}$ e a escala de diferença de massa que controla a oscilação neste caso não é a detectada nos experimentos. Por isso, esta forma é descartada. 
Existe, porém, outra simplificação que podemos fazer. A distância entre o Sol e a Terra, ou nos experimentos terrestres entre a fonte e o detector, é em geral muito superior ao comprimento $L_{31}=4 \pi E / \Delta m_{31}^{2}$, o que leva ao seguinte resultado $[68,176]$ :

$$
P_{e e} \simeq \sin ^{4} \theta_{13}+\cos ^{4} \theta_{13} P_{2}\left(\Delta m_{21}^{2}, \theta_{12}\right) .
$$

No caso de neutrinos solares devemos levar em consideração o efeito de matéria, mas como $\Delta m_{31}^{2} \gg 2 E V(x)$ basta utilizar um potencial efetivo $V(x) \rightarrow$ $V(x) \cos ^{2} \theta_{13}$ para $P_{2}$ na matéria da Eq. (8.46).

\section{Inclusão de $\Delta m_{21}^{2}$ na análise de neutrinos atmosféricos}

Para obter a aproximação da análise de neutrinos atmosféricos fizemos $\frac{\Delta m_{21}^{2}}{\Delta m_{31}^{2}} \rightarrow 0$. Novamente, a maneira mais simples de introduzir de volta o efeito de $\Delta m_{21}^{2}$ na análise é considerar $\theta_{13} \rightarrow 0$, como feito no caso anterior. Entretanto devemos calcular $P_{\mu \mu}$ que pode depender das duas escalas de diferenças de massa. De fato, obtemos [68]

$$
P_{\mu \mu} \simeq 1-c_{23}^{2} P_{2}-\frac{\sin ^{2} 2 \theta_{23}}{2}\left[1-\sqrt{1-P_{2} \cos \varphi}\right]
$$

$\operatorname{com} \varphi \approx\left(\Delta m_{31}^{2}+s_{12}^{2} \Delta m_{21}^{2}\right) x / 2 E$.

\section{Inclusão de $\theta_{13}$ na análise de neutrinos atmosféricos}

A inclusão de $\theta_{13}$ na análise de neutrinos atmosféricos é mais simples, basta considerar o efeito de matéria com densidade constante na aproximação $\frac{\Delta m_{21}^{2}}{\Delta m_{31}^{2}} \rightarrow 0$ [68]. Assim obtemos

$$
P_{\mu \mu} \simeq 1-\sin ^{2} 2 \tilde{\theta}_{13} s_{23}^{4} S_{31}-\sin ^{2} \tilde{\theta}_{13} \sin ^{2} 2 \theta_{23} S_{21}-\cos ^{2} \tilde{\theta}_{13} \sin ^{2}{ }_{23} S_{32},
$$

onde $\sin 2 \tilde{\theta}_{13}=\frac{\sin 2 \theta_{13}}{\sqrt{\left(\cos 2 \theta_{13}-2 E V / \Delta m_{31}^{2}\right)^{2}+\sin ^{2} 2 \theta_{13}}}$, e os fatores oscilantes $S_{i j}=\sin ^{2} \frac{\Delta \mu_{i j}^{2}}{4 E} x$ são dados com

$$
\begin{aligned}
\Delta \mu_{21}^{2} & =\frac{\Delta m_{31}^{2}}{2}\left(\frac{\sin 2 \theta_{13}}{\sin 2 \tilde{\theta}_{13}}-1\right)-E V \\
\Delta \mu_{32}^{2} & =\frac{\Delta m_{31}^{2}}{2}\left(\frac{\sin 2 \theta_{13}}{\sin 2 \tilde{\theta}_{13}}+1\right)+E V, \\
\Delta \mu_{31}^{2} & =\frac{\Delta m_{21}^{2}}{2} \frac{\sin 2 \theta_{13}}{\sin 2 \tilde{\theta}_{13}}
\end{aligned}
$$

que se reduz ao caso utilizado na Eq. (8.13) quando a densidade é nula. 


\section{Conclusões e perspectivas}

No final da década passada e início desta, a física de neutrinos recebeu grandes contribuições dos dados experimentais cada vez mais precisos que acabaram por confirmar o fenômeno de conversão de sabor no setor, apontando firmemente para a oscilação induzida por massa como explicação. Futuros experimentos estão prestes à entrar em operação e uma nova geração de dados experimentais já começarão a existir com precisão potencial de levar a determinação dos parâmetros de oscilação até ordem de poucos por centos, almejando assim também medir os valores dos parâmetros de oscilação $\theta_{13}$ e $\delta$ desconhecidos até o presente momento.

Nesta conclusão descrevemos primeiro o que foi apresentado nos capítulos introdutórios do fenômeno de oscilação de neutrinos. Depois disso comentaremos os resultados principais de nosso estudo mostrado nos capítulos finais. Além disso discutimos as perspectivas de aplicação de nosso trabalho.

No primeiro capítulo relacionamos o desenvolvimento da idéia de neutrinos e suas propriedades com a criação da teoria eletrofraca do MP. Acreditamos na importância de relacionar ambas histórias para exemplificar a influência que as características dos neutrinos exerceram e ainda exercem no desenvolvimento dos modelos que descrevem as partículas e suas interações.

No segundo capítulo apresentamos as principais fontes de neutrinos disponíveis para o homem realizar experimentos. Complementamos o capítulo com uma descrição das principais técnicas de detecção empregadas até hoje. É imprescindível um conhecimento da distribuição energética das partículas estudadas e das formas que podemos detectá-las. Portanto, no Capítulo 3 decidimos descrever da maneira mais completa possível sobre os experimentos que de alguma forma se relacionam com a observação de oscilação de neutrinos. É importante conhecer quais as idéias já realizadas e em que posição se encontram as medidas do que vamos estudar.

O Capítulo 4 introduz o modelo teórico de análise dos resultados previamente apresentados fornecendo os valores mais prováveis dos parâmetros de oscilação. Desta forma ele indica de maneira concisa onde e como será utilizado nosso estudo, apontando evidentemente na direção em que devemos prosseguir. Com 
este capítulo fechamos a parte introdutória da dissertação.

O Capítulo 5 já engloba a parte teórica do estudo aqui realizado. Ele apresenta os tipos de termos de massa e seus mecanismos de geração para neutrinos. Concluimos o capítulo com a observação de que o estudo de oscilação não evidencia o tipo de massa, Dirac ou Majorana, porque o fenômeno não viola a conservação do número leptônico (somente de sabor). Além disto as medidas dos parâmetros de oscilação são úteis para descartar modelos de mecanismos de geração de massa.

No Capítulo 6 demonstramos que a equação de evolução dos neutrinos com o potencial devido à densidade de neutrinos na matéria, $N_{e}$, é dada sob certas condições por:

$$
i \frac{d}{d x}|\nu\rangle=\left[\frac{1}{2 E} U \operatorname{diag}\left(m_{1}^{2}, m_{2}^{2}, m_{3}^{2}\right) U^{\dagger}+V(x) \operatorname{diag}(1,0,0)\right]|\nu\rangle,
$$

onde $V(x)=\sqrt{2} G_{F} N_{e}(x)$ e $|\nu\rangle=\left(\nu_{e} \nu_{\mu} \nu_{\tau}\right)^{T}$. Detalhamos um estudo sobre a parametrização da matriz de mistura até chegar na tradicional

$$
U=\left(\begin{array}{ccc}
c_{12} c_{13} & s_{12} c_{13} & s_{13} e^{-i \delta} \\
-s_{12} c_{23}-c_{12} s_{13} s_{23} e^{i \delta} & c_{12} c_{23}-s_{12} s_{13} s_{23} e^{i \delta} & c_{13} s_{23} \\
s_{12} s_{23}-c_{12} s_{13} c_{23} e^{i \delta} & -c_{12} s_{23}-s_{12} s_{13} c_{23} e^{i \delta} & c_{13} c_{23}
\end{array}\right) .
$$

As demonstrações foram suficientemente rigorosas para sanar qualquer dúvida existente nos limites de sua validade e maneiras de ir além. Apresentamos no final o importante resultado de que em três famílias apenas duas fórmulas de probabilidade precisam ser encontradas, $P_{e \mu}$ e $P_{\mu \tau}$, sendo as outras sistematicamente deduzidas a partir delas.

No Capítulo 7 apresentamos todas as soluções exatas conhecidas para as equações de evolução acima. Nós propusemos um formalismo capaz de resolver a equação de evolução sistematicamente para todos os tipos de potenciais cujas soluções eram conhecidas em duas e três gerações. O resultado principal generalizado obtido foi que as soluções para $N$ famílias de neutrinos podem ser deduzidas através apenas do conhecimento da solução $\psi$ da seguinte equação:

$$
\prod_{j=1}^{N}\left(i \frac{d}{d u}-\mu_{j}\right) \psi=x_{0} V f(u) \prod_{j=2}^{N}\left(i \frac{d}{d u}-\omega_{j}\right) \psi
$$

onde $f(u)$ dá a forma do potencial e $N=2,3$.

Através dos polinômios $P_{\mu}(t)$ e $P_{\omega}(t)$ cujas raízes são $\mu_{j}$ e $\omega_{k}$, vemos que a forma polinomial nas derivadas em que a equação diferencial se apresenta sugere a resolução do problema por transformada de Fourier. Evidentemente 
este método só é interessante quando $f$ também é um polinômio. Sendo assim, em duas gerações, o método só tem utilidade se $f(u)=\frac{a_{1}+b_{1} u}{a_{2}+b_{2} u}$, o que chamamos de caso linear generalizado. Este caso cobre as soluções conhecidas de $V(x) \propto x$ e $1 / x$. Acima de duas gerações o procedimento realizado é o mesmo, o que significa que também encontramos soluções para o caso linear generalizado em três gerações. Aparentemente a transformada de Fourier também deveria ser útil para $f$ de segundo grau. Entretanto a equação obtida neste caso não possui solução analítica exata. Desta forma esgotamos os potenciais cujas formas tem solução exata empregando a transformada de Fourier.

Por outro lado podemos tentar chegar numa equação diferencial para as chamadas funções especiais (Bessel, Whittaker, Weber e outras, assim como os polinômios de Jacobi, Legendre e outros), que era o método utilizado na literatura para as soluções conhecidas. Essas funções especiais costumam ser soluções de uma equação linear de segunda ordem. De fato, na Ref. [207], o autor demonstra que essas funções especiais decorrem todas de limites ou casos particulares da função hipergeométrica

$$
{ }_{2} F_{1}(a, b ; c ; z)=\sum_{k=0}^{\infty} \frac{(a)_{k}(b)_{k}}{(c)_{k}} \frac{z^{k}}{k !}
$$

onde $(a)_{k}=\frac{\Gamma(a+k)}{\Gamma(a)}=a(a+1) \cdots(a+k-1)$, que é solução da chamada equação hipergeométrica:

$$
\left[z(1-z) \frac{d^{2}}{d z^{2}}+[c-(a+b+1) z] \frac{d}{d z}-a b\right]{ }_{2} F_{1}=0
$$

Esta equação por sua vez é um caso particular da equação hipergeométrica generalizada

$$
z \frac{d}{d z} \prod_{j=1}^{q}\left(z \frac{d}{d z}+b_{j}-1\right) F=z \prod_{k=1}^{p}\left(z \frac{d}{d z}+a_{k}\right) F,
$$

cuja solução é

$$
{ }_{p} F_{q}\left[\begin{array}{ccc}
a_{1}, & \ldots, & a_{p} \\
b_{1}, & \ldots, & b_{q}
\end{array} ; z\right]=\sum_{k=0}^{\infty} \frac{\left(a_{1}\right)_{k} \cdots\left(a_{p}\right)_{k}}{\left(b_{1}\right)_{k} \cdots\left(b_{q}\right)_{k}} \frac{z^{k}}{k !}
$$

Não é difícil notar a semelhança entre esta equação e aquela obtida para $\psi$, com excessão de um caso em duas gerações, a mudança de variável necessária para transformar uma na outra é $z \propto e^{-u}$ e $f(u)=\frac{a_{1}+b_{1} e^{-u}}{a_{2}+b_{2} e^{-u}}$, potencial que chamamos de exponencial generalizado.

A excessão em duas gerações é o caso linear generalizado, que sem nenhuma mudança de variável, pode ser rearranjado para mostrar que neste caso a equação 
já é uma hipergeométrica confluente. Isto explica o porquê em duas gerações a resolução por transformada de Fourier nos leva à soluções dadas por funções especiais.

O caso exponencial generalizado engloba os conhecidos $V(x) \propto e^{-x}$ e $1+$ $\tanh x$, além de trazer solução para outros $\operatorname{como} \tanh x$ e tan $x$. Além disso, notamos que existem três parâmetros livres: $f(u)=\alpha_{1} \frac{1+\alpha_{2} e^{-u}}{1+e^{-u}}$ e $V_{0}$. É possível que haja potenciais físicos que possam ser ajustados com essa parametrização. Esse método pode então ser uma alternativa aos métodos de aproximação. Até então apenas potenciais com dois parâmetros livres possuíam soluções exatas na literatura para o problema de oscilação de neutrinos na matéria.

Acreditamos que a partir de agora fica difícil pensar numa outra forma de encontrar soluções exatas, pois os métodos existentes devem nos levar à uma solução com representação integral (transformada de Fourier), ou de alguma maneira simplificar a equação levando-a à alguma conhecida (cujas soluções são funções especiais). Entretanto quase todas as funções especiais bem estudadas são casos particulares da hipergeométrica generalizada. Para as que restam (Mathieu e outras bem menos conhecidas), podemos procurar os potenciais que dariam origem à tais equações, e estes não devem ser simples e por vezes não resolúveis analiticamente (pois para encontrar o potencial a partir da solução, no caso de oscilação de neutrinos na matéria, temos que resolver uma equação diferencial não linear).

Portanto acreditamos ter agora uma compreensão mais completa das possibilidades de soluções analíticas para as equações de evolução de neutrinos na matéria.

No último capítulo, descrevemos os métodos de procura por soluções aproximadas da equação de evolução. A aproximação adiabática foi extensamente apresentada por ser muito utilizada nas análises dos dados. Procuramos, nesse capítulo, apresentar a demonstração do método de Landau e do Ansatz de Kuo e Pantaleone. Discutimos também uma outra solução com grande região de aplicação (fora da zona de ressonância) e de grande precisão. Explicamos a situação atual das fórmulas aproximadas em três gerações.

O estudo realizado foi motivado pela crescente precisão que os experimentos de oscilação têm atingido e a necessidade de entender melhor o papel da matéria em três gerações genuínas. Acreditamos que o formalismo desenvolvido aqui abre caminho para estudo de novos potenciais e também para uma análise mais profunda das soluções obtidas para outros domínios de aplicações. 


\section{Apêndice A}

\section{Sobre a helicidade e a quiralidade de partículas}

Para uma partícula sem massa, os estados de quiralidade e helicidade são os mesmos, e além disso, conservados ao longo da evolução. Ao passar para uma partícula massiva, estes diferem, e no caso de neutrinos, um neutrino (antineutrino) de mão direita (esquerda) deveria existir, indo além do Modelo Padrão. O objetivo desta seção é demonstrar a seguinte expansão de helicidade em termos da quiralidade para uma partícula de massa pequena:

Seja $\pi(h)$ o operador de helicidade de uma particula de spin $1 / 2$ de energia positiva, e $P_{R}=\frac{1+\gamma_{5}}{2}$ o operador quiralidade de mão direita da mesma partícula, $m$ sua massa e $p=\left(\sqrt{|\vec{p}|^{2}+m^{2}}, \vec{p}\right)$ o seu quadrimomento, então:

$$
\pi(h)=\frac{1+\gamma_{5}}{2}+m \frac{\gamma_{5} \not p^{\prime}}{4|\vec{p}|^{2}}+O\left(\frac{m^{3}}{|\vec{p}|^{4}}\right)
$$

onde $\not p=p_{i} \gamma^{i}$ e $p^{\prime}=(-|\vec{p}|, \vec{p})$.

De fato, podemos definir os dois operadores, helicidade e quiralidade, como segue:

- Helicidade: projeção do spin sobre o momento da partícula. No referencial de repouso, é fácil de ver que a projeção do spin sobre um eixo qualquer $\vec{n}$ é

$$
\pi(\vec{n})=\frac{1+\vec{\Sigma} \cdot \vec{n}}{2}=\frac{1+\gamma_{5} \not h}{2}
$$

$\operatorname{com} \vec{\Sigma}=\gamma_{5} \gamma^{0} \vec{\gamma}$ e $n=(0, \vec{n})$. Ao executar uma transformação de Lorentz, $n^{\prime}=\Lambda n$ vê-se que $\pi$ não é invariante de Lorentz. No caso da helicidade toma-se $\vec{n}=\vec{p} /|\vec{p}|$. Passando para um referencial qualquer, obtém-se

$$
\overrightarrow{n^{\prime}}=\left(\frac{|\vec{p}|}{m}, \frac{p^{0} \vec{p}}{m|\vec{p}|}\right)
$$


e finalmente

$$
\pi(h)=\frac{1+\gamma_{5} \not h}{2}=\frac{1+\vec{\Sigma} \cdot \vec{p} /|\vec{p}|}{2},
$$

onde a última igualdade é tomada para definição de helicidade de partículas sem massa. Também é fácil de verificar que $\pi$ é um projetor, afinal $\pi(n)^{2}=$ $\pi(n)$ e $\pi(n)+\pi(-n)=1$.

- Quiralidade: é dita como sendo a reflexão por um espelho. Os operadores de projeção que podem ser definidos e invariantes de Lorentz são

$$
P_{R, L}=\frac{1 \pm \gamma_{5}}{2}
$$

Para partículas massivas de spin $1 / 2$, os operadores de projeção de energia positiva e negativa são $\Lambda_{ \pm}=\frac{ \pm \not p+m}{2 m}$. Olhando para a helicidade de uma partícula de energia positiva no limite de massas pequenas ou altas energias $m / p^{0} \rightarrow 0$, tem-se $p^{0}=\sqrt{|\vec{p}|^{2}+m^{2}}=|\vec{p}|+\frac{m^{2}}{2|\vec{p}|}+O\left(m^{4} /|\vec{p}|^{3}\right)$ e $n^{\mu}=\frac{p^{\mu}}{m}+\frac{m}{2|\vec{p}|^{2}}(-|\vec{p}|, \vec{p})+O\left(m^{3} /|\vec{p}|^{4}\right)$, de onde vemos que

$$
\pi(h) \Lambda_{+}=\frac{1+\gamma_{5}}{2} \Lambda_{+}+m \frac{\gamma_{5} \not p^{\prime}}{4|\vec{p}|^{2}} \Lambda_{+}+O\left(\frac{m^{3}}{|\vec{p}|^{4}}\right) .
$$

Para partículas sem massa, a equação de Dirac é $i \not \partial \psi=0$, multiplicando a equação por $\gamma_{5} \gamma^{0}$ chega-se à $i \vec{\Sigma} \cdot \vec{\nabla} \psi=-i \gamma_{5} \partial_{0} \psi$. A solução de onda plana com energia positiva é da forma $\psi(x)=\psi(p) e^{-i p x}$, e como $p^{0}=|\vec{p}|$ então a equação se transforma em

$$
\frac{\vec{\Sigma} \cdot \vec{p}}{|\vec{p}|} \psi=\gamma_{5} \psi,
$$

ou seja, $\pi(h)=P_{R}$.

A conservação da quiralidade pode ser vista utilizando novamente a equação de Dirac, $(i \not \partial-m) \psi=0$, aplicando os operadores de projeção de quiralidade obtém-se $i \not \partial P_{L, R} \psi=m P_{R, L} \psi$, que diz claramente que se $m \rightarrow 0$ as quiralidades evoluem independetemente.

Conclui-se assim que para partículas de energia positiva, o operador de helicidade tende ao operador de quiralidade de mão direita quando a massa vai à zero. Analogamente se a energia for negativa, o operador de helicidade tenderá ao de quiralidade de mão esquerda. 


\section{Apêndice B}

\section{Neutrinos de Majorana}

O campo que descreve os férmions carregados é o de Dirac. Estas partículas obedecem a equação de Dirac e são descritas por espinores de quatro componentes. Este tópico é muito bem trabalhado em todos os cursos de Teoria Quântica de Campos. Entretanto, partículas sem massa ou sem carga elétrica podem ser descritas por espinores de duas componentes. No primeiro caso, tratam-se de espinores de Weyl, enquanto que no segundo, denomina-se espinores de Majorana. Pela pouca exposição destes em cursos tradicionais, e dada a importância dos mesmos no tratamento fenomenológico de neutrinos, este apêndice foi criado.

O intuito deste apêndice é apresentar a diferença existente nos termos de massa para campos de Dirac e de Majorana. Portanto supõe-se que neutrinos são massivos e desta forma não podem ser descritos por espinores de Weyl.

\section{B.1 Definição do campo de Majorana}

A maneira mais física de se pensar numa partícula de Majorana é dizendo que esta é uma partícula fermiônica idêntica à sua antipartícula. Deste modo introduzimos um operador de conjugação partícula-antipartícula, $C$, e igualamos a partícula com seu campo conjugado

$$
\psi=e^{i \eta} \psi^{c} \equiv e^{i \eta} C \gamma_{0} \psi^{*},
$$

que é uma condição física válida em qualquer referencial, diferente do caso mais simples $\psi=\psi^{*}$. Para tanto, $C$ é definido pela relação $C \gamma_{0} \sigma_{\mu \nu}^{*}=-\sigma_{\mu \nu}^{*} C \gamma_{0}$, cuja forma depende da representação das matrizes $\gamma$. Na representação quiral $C=i \gamma_{2} \gamma_{0}$. A fase que aparece pode ser absorvida na definição do campo e assim $\eta=0$ pode ser escolhido, porém esta liberdade é conveniente em alguns casos. Ela é útil na demonstração que $C \gamma_{0}$ é equivalente à $\gamma_{0} C$ na definição do campo de Majorana. 
Uma definição alternativa é baseada na busca de uma equação relativística que descreve um férmion massivo de 2 componentes espinoriais. Um método é completar a equação de Weyl com um termo de massa da seguinte forma

$$
i\left(\partial_{0}-\vec{\sigma} \cdot \vec{\nabla}\right) \psi-i m \sigma_{2} \psi^{*}=0
$$

onde trabalhamos na representação quiral, $\psi=\psi_{L}$. Através de um procedimento não trivial, com a inclusão de váriaveis de Grassmann, podemos mostrar que esta equação decorre da densidade lagrangeana

$$
\mathcal{L}_{M}=\psi^{\dagger}\left(\partial_{0}-\vec{\sigma} \cdot \vec{\nabla}\right) \psi+\frac{i m}{2}\left(\psi^{T} \sigma_{2} \psi-\psi^{\dagger} \sigma_{2} \psi^{*}\right)
$$

Esta é a lagrangeana livre de uma partícula de Majorana.

No MP os campos de mão esquerda se transformam como dubletos de $S U(2)$. Desta forma o campo conjugado de carga é $\Psi^{c}=\gamma_{0} C i \sigma_{2} \Psi^{*}$, pois $\Psi^{*}$ não se transforma como um dubleto: $\Psi \rightarrow e^{i \frac{\vec{\sigma} \cdot \vec{\theta}}{2}} \Psi$, mas $i \sigma_{2} \Psi^{*} \operatorname{sim}$.

\section{B.2 Propriedades}

Algumas propriedades do operador $C$ podem ser obtidas se impusermos algumas condições no campo conjugado. Se $\psi^{c}$ é normalizado quando $\psi$ o é, usando a definição de $C$, e assumindo $\left(\psi^{c}\right)^{c}=\psi$, que é bem natural, obtém-se as relações

$$
C^{\dagger}=C^{T}=C^{-1}=-C \quad \text { e } \quad C^{-1} \gamma_{\mu} C=-\gamma_{\mu}^{T}
$$

Como consequência destas seguem as relações

$$
\overline{\psi^{c}}=\psi^{T} C, \quad \overline{\psi_{1}} \psi_{2}^{c}=\overline{\psi_{2}^{c}} \psi_{1} \quad \text { e } \quad \overline{\psi_{1}} A \psi_{2}=\overline{\psi_{2}^{c}}\left(c^{-1} A^{T} C\right) \psi_{1}^{c}
$$

Na representação quiral obtém-se

$$
\left(\psi_{L}\right)^{c}=\left(\psi^{c}\right)_{R}, \quad\left(\psi_{R}\right)^{c}=\left(\psi^{c}\right)_{L} \quad \text { e } \overline{\psi_{L}} \psi_{R}=\overline{\left(\psi_{R}\right)^{c}}\left(\psi_{L}\right)^{c}
$$

\section{B.3 Propagador de Majorana}

Assim como a extensão do propagador do campo escalar para o complexo é igual ao do campo escalar real, o campo de Majorana tem propagador igual ao de Dirac:

$$
S(\not p)=\frac{i}{\not p-m}
$$




\section{B.4 Termos de massa para neutrinos}

Para neutrinos, caso incluamos um neutrino esteril de mão direita no MP, podemos construir a partir dos campos $\psi_{L}$ e $\psi_{R}$, dois novos campos satisfazendo a condição de Majorana: $\chi=\psi_{L}+\psi_{L}^{c}$ e $\omega=\psi_{R}+\psi_{R}^{c}$. Assim seus termos de massa se escrevem

$$
\mathcal{L}_{L}^{M}=-\frac{1}{2} \bar{\chi} m_{L} \chi=-\frac{m_{L}}{2}\left(\overline{\psi_{L}^{c}} \psi_{L}+\text { h.c. }\right)
$$

e

$$
\mathcal{L}_{R}^{M}=-\frac{1}{2} \bar{\omega} m_{R} \omega=-\frac{m_{R}}{2}\left(\overline{\psi_{R}^{c}} \psi_{R}+\text { h.c. }\right),
$$

onde o fator $\frac{1}{2}$ aparece devido ao termo cinético da lagrangeana de Majorana. Notamos que os termos de Majorana violam o número leptônico total por duas unidades. 


\section{Apêndice C}

\section{Glossário}

CC - Corrente carregada

EDQ - Eletrodinâmica Quântica

ES - Espalhamento elástico

FBE - Feixe de Banda Estreita

FBL - Feixe de Banda Larga

FC - "Fully Contained"

FFE - Feixe Fora do Eixo

LBL - "Long BaseLine"

LHC - "Large Hadron Collider"

MC - Monte Carlo

MP - Modelo Padrão

MQ - Mecâninca Quântica

MSP - Modelo Solar Padrão

NC - Corrente neutra

PC - "Partially Contained"

SBL - "Short BaseLine"

SK - Super-Kamiokande

ST-UG - "Stopping UpGoing muon" 
TFM - Tubo Fotomultiplicador

TH-UG - "Through UpGoing muon"

UG - "UpGoing muon"

vev - valor esperado de vácuo 


\section{Referências Bibliográficas}

[1] Christine Sutton, em "Spaceship neutrino", Cambridge University Press, 1992.

[2] H. Becquerel, Compt. Ren. 122, 501 (1896) - cinco artigos foram publicados na mesma edição, veja também as páginas 559, 689, 762 e 1086.

[3] J. Chadwick, Verhandl Dtsch. Phys. Ges. 16, 383 (1914).

[4] C. D. Ellis e W. A. Wooster, Proc. Roy. Soc. A 117, 109 (1927).

[5] N. Bohr, em uma carta à W. Pauli, tradução em inglês: Niels Bohr Collected Works (North Holland, Amsterdam, 1986), vol 6, ed. U. Hoyer, p. 443.

[6] W. Pauli, Open Letter to Radioactive Persons, 1930, para tradução em inglês veja: Physics Today 31, 27 (1978).

[7] W. Pauli, em Septième Conseil de Physique, Solvay (Gauthier-Villars, Paris, 1934), p. 324.

[8] E. Fermi, Z. Phys. 88, 161 (1934); Nuovo Cim. 11, 1 (1934).

[9] R. Peierls, Cont. Phys. 24, 221 (1983).

[10] F. Reines e C. L. Cowan Jr., Phys. Rev. 90, 492 (1953); C. L. Cowan Jr., F. Reines, F. B. Harrison, E. C. Anderson e F. N. Hayes, Phys. Rev 90, 493 (1953).

[11] C. L. Cowan Jr., F. Reines, F. B. Harrison, H. B. Kruse e A. D. McGuire, Science 124, 103 (1956); F. Reines e C. L. Cowan Jr., Nature 178, 446 (1956).

[12] T. D. Lee e C. N. Yang, Phys. Rev. 104, 254 (1956).

[13] W. Pauli, em uma carta à V. F. Weisskopf, tradução em inglês: Collected Scientific Papers by Wolfgang Pauli (Wiley Interscience, New York, 1964), vol 1, ed. R. Kronig e V. F. Weisskopf, p. xiii. 
[14] A. Salam, Nobel Lecture, 8 de dezembro 1979. The Nobel Foundation 1980.

[15] C. S. Wu et al., Phys. Rev. 105, 1413 (1957).

[16] M. Goldhaber, L.Grodzins, e A. W. Sunyar, Phys. Rev. 109, 1015 (1958).

[17] R. P. Feynman, Phys. Rev. 74, 1930 (1948); Phys. Rev. 76, 769 (1949); J. Schwinger, Phys. Rev. 74, 1439 (1948); Phys. Rev. 75, 651 (1949); T. Tati e S. Tomonaga, Prog. Theor. Phys. 3, 391 (1948); veja também: Z. Koba, T. Tati e S. Tomonaga, Prog. Theor. Phys. 2, 101 e 198 (1947).

[18] J. Schwinger, Ann. Phys. 2, 407 (1957); T. D. Lee e C. N. Yang, Phys. Rev. 108, 1611 (1957).

[19] S. L. Glashow, Nucl. Phys. 22, 579 (1961); A. Salam e J. C. Ward, Phys. Lett. 13, 168 (1964); S. Weinberg, Phys. Rev. Lett. 19, 1264 (1967); A. Salam, em "Elementary Particle Theory", The Nobel Symposium no 8, editado por N. Svartholm (Almqvist e Wiksell, Stackholm, 1968), p. 367.

[20] G. 't Hooft, Nucl. Phys. B 33, 173 (1971); Nucl. Phys. B 35, 167 (1971).

[21] P. W. Higgs, Phys. Lett. 12, 132 (1964).

[22] R. N. Mohapatra e P. Pal, em "Massive neutrinos in physics and astrophysics", World Scientific, 2005.

[23] W.-M. Yao et al. [Particle Data Group], J. Phys. G 33, 1 (2006) [http://pdg.lbl.gov].

[24] ALEPH, http://aleph.web.cern.ch/aleph/, baseado nos dados do LEP até 1993; P. Abreu et al., Z. Phys. C 74, 577 (1997).

[25] N. Jarosik et al., astro-ph/0603449.

[26] Mainz Collab., J. Bonn et al., Nucl. Phys. Proc. Suppl. 91, 273 (2001).

[27] K. A. Assamagan et al., Phys. Rev. D 53, 6065 (1996).

[28] R. Barate et al., Eur. Phys. J. C 1, 395 (1998).

[29] H. Klapdor-Kleingrothaus e S. Stoica, em "Double-beta decay and related topics", World Scientific, 1995.

[30] S. R. Elliot, A. A. Hahn, M. K. Moe, Phys. Rev. Lett. 59, 2020 (1987).

[31] J. Bahcall, Phys. Rev. Lett. 12, 300 (1964). 
[32] Raymond Davis Jr., D. Harmer e K. Hoffman, Phys. Rev. Lett. 20, 1205 (1968).

[33] Para uma coletânea de todos os modelos solares, veja o sítio http://www.sns.ias.edu/ jnb/ .

[34] B. Pontecorvo, J. Exptl. Theoret. Phys. 33, 549 (1957); B. Pontecorvo, J. Exptl. Theoret. Phys. 34, 247 (1958).

[35] Z. Maki, M. Nakagawa e S. Sakata, Prog. Theor. Phys. 28, 870 (1962).

[36] V. Gribov e B. Pontecorvo, Phys. Lett. B 28, 493 (1969).

[37] S. P. Mikheev e A. Yu. Smirnov, Sov. J. Nucl. Phys. 42, 913 (1985); S. P. Mikheyev e A. Yu. Smirnov, Nuovo Cimento C 9, 17 (1986).

[38] L. Wolfenstein, Phys. Rev. D 17, 2369 (1978).

[39] E. Majorana, Nuovo Cimento 14, 171 (1937).

[40] J. Chadwick, Proc. Roy. Soc. A 136, 692 (1932).

[41] B. Pontecorvo, em "Inverse $\beta$ Process", Chalk River Report PD-205, novembro de 1946 (não publicado).

[42] S. Sakata e T. Inounë, Prog. Theor. Phys. 1, 143 (1946).

[43] B. Pontecorvo, Phys. Rev. 72, 246 (1947).

[44] A. Salam, Nuovo Cim. 5, 299 (1957); T. D. Lee e C. N. Yang, Phys. Rev. 105, 1671 (1957); L. D. Landau, Zh. Eksp. Teor. Fiz. 32, 407 (1957); veja também JETP 5, 337 (1957).

[45] G. Danby, J. M. Gaillard, K. Goulianos, L. M. Lederman, N. Mistry, M. Schwartz e J. Steinberger, Phys. Rev. Lett. 9, 36 (1962).

[46] F. Englert e R. Brout, Phys. Rev. Lett. 13, 321 (1964); G. S. Guralnik, C. R. Hagen, e T. W. B. Kibble, Phys. Rev. Lett. 13, 585 (1964).

[47] Raymond Davis Jr., Phys. Rev. Lett. 12, 303 (1964).

[48] M. L. Perl et al., Phys. Rev. Lett. 35, 1489 (1975).

[49] K. Hirata et al. (Kamiokande), Phys. Rev. Lett. 58, 1490 (1987).

[50] G. S. Abrams et al. (MARK-II Collab.), Phys. Rev. Lett. 63, 2173 (1989). 
[51] LEP Collaborations: ALEPH, DELPHI, L3 e OPAL, Phys. Lett. B 276, 247 (1992).

[52] Y. Fukuda et al. (Super-Kamiokande), Phys. Rev. Lett. 81, 1562 (1998) [hep-ex/9807003].

[53] Q. R. Ahmad et al. [SNO Collaboration], Phys. Rev. Lett. 87, 071301 (2001); ibid. 89, 011301 (2002); ibid. 89, 011302 (2002).

[54] K. Kodama et al., Phys. Lett. B 504, 218 (2001).

[55] A nova força dos neutrinos, Revista Pesquisa Fapesp, 45 (1999).

[56] J. N. Bahcall, M. H. Pinsonneault, e S. Basu, Astrophys. J. 555, 990 (2001), astro-ph/0010346.

[57] J. N. Bahcall, http://sns.ias.edu/ jnb.

[58] J. N. Bahcall, A. Serenelli, e S. Basu, Astrophys. J. 621, L85 (2005) [astro$\mathrm{ph} / 0412440]$.

[59] M. Honda et al., Phys. Lett. B 248, 193 (1990); M. Honda et al., Phys. Rev. D 52, 4985 (1995); M. Honda et al., Prog. Theor. Phys. Suppl. 123, 483 (1996); G. Fiorentini et al., Phys. Lett. B 510, 173 (2001).

[60] T.K. Gaisser et al., Phys. Rev. D 38, 85 (1988); G. Barr et al., Phys. Rev. D 39, 3532 (1989); V. Agraval et al., Phys. Rev. D 43, 1314 (1996); P. Lipari et al., Phys. Rev. D 58, 073005 (1998).

[61] J. A. Simpson, Ann. Rev. Nucl. and Part. Sci. 33, 323 (1983).

[62] G. Raffelt, em "Stars as laboratories for Fundamental Physics", Chicago University Press (1996).

[63] A. Burrows et al., astro-ph/0510687.

[64] E. Sanshiro, Tese de Doutorado: "Neutrino Geophysics and Observation of GeoNeutrinos at KamLAND", Tohoku University, (2005), http://kamland.stanford.edu/GeoNeutrinos/GeoNuResults.

[65] J. M. Irvine e R. Humphreys, J. Phys. G:Nucl. Phys 9, 847 (1983).

[66] T. Weiler, Astropart. Phys. 11, 303 (1999) [hep-ph/9710431]; D. Fargion, B. Mele, e A. Salis, Astrophys. J. 517, 725 (1999) [astro-ph/9710029].

[67] P. Migliozzi, hep-ph/0311269. 
[68] M. C. Gonzalez-Garcia e M. Maltoni, hep-ph/0704.1800.

[69] P. Vogel e J. Engel, Phys. Rev. D 39, 3378 (1989).

[70] Y. Declais et al., Phys. Lett B 338, 383 (1994); ibid. B 434, 503 (1995).

[71] F. Boehm e P. Vogel, em "The Physics of Massive Neutrinos", Cambridge University Press (1992).

[72] K. Nakajima et al., physics/0607126.

[73] K. Kleinknecht, em "Detectors for Particle Radiation", Cambridge University Press (2003).

[74] G. Giacomelli e M. Giorgini, hep-ex/0504002.

[75] MINOS Collaboration, http://www-numi.fnal.gov/ .

[76] D. Ayres et al. [NOvA Collaboration], hep-ex/0210005; ibid, hepex/0503053.

[77] C. Giunti e M. Laveder, hep-ph/0301276.

[78] B. T. Cleveland et al., Astrophys. J. 496, 505 (1998).

[79] J. N. Abdurashitov et al. [SAGE Collaboration], J. Exp. Theor. Phys. 95, 181 (2002) [Zh. Eksp. Teor. Fiz. 122, 211 (2002)][astro-ph/0204245].

[80] W. Hampel et al. [GALLEX Collaboration], Phys. Lett. B 447, 127 (1999).

[81] M. Altmann et al. [GNO Collaboration], Phys. Lett. B 616, 174 (2005) [hep-ex/0504037].

[82] M. Altmann et al. [GNO Collaboration], Phys. Lett. B 490, 16 (2000) [hep-ex/0006034].

[83] Y. Fukuda et al. [Kamiokande Collaboration], Phys. Rev. Lett. 77, 1683 (1996).

[84] M. B. Smy et al. [Super-Kamiokande Collaboration], Phys. Rev. D 69, 011104 (2004) [hep-ex/0309011].

[85] Y. Fukuda et al., Phys. Lett. B 539, 179 (2002) [hep-ex/0205075].

[86] S. N. Ahmed et al. [SNO Collaboration], Phys. Rev. Lett. 92, 181301 (2004); B. Aharmim et al., Phys. Rev. C 72, 055502 (2005).

[87] L. Oberauer, Nucl. Phys. Proc. Suppl. 77, 48 (1999). 
[88] M. Nakahata, Nucl. Phys. Proc. Suppl. 145, 23 (2005).

[89] UNO Proto-collaboration, UNO Whitepaper: Physics Potential and Feasibility of UNO, SBHEP-01-03 (2000); C. K. Jung, AIP Conf. Proc. 533, 29 (2000) [hep-ex/0005046].

[90] Y. Itow et al., hep-ex/0106019.

[91] K. Nakamura, Int. J. Mod. Phys. A 18, 4053 (2003).

[92] Working Group of Neutrino Physicists, Whitepaper: Mesuring theta13 with a reactor experiment (2004).

[93] J. F. Cavaignac et al., Phys. Lett. B 148, 387 (1984).

[94] M. Apollonio et al. [CHOOZ Collaboration], Phys. Lett. B 466, 415 (1999) [hep-ex/9907037].

[95] A. Piepke [Palo Verde Collaboration], Prog. Part. Nucl. Phys. 48, 113 (2002).

[96] A. Piepke [KamLAND Collaboration], Nucl. Phys. Proc. Suppl. 91, 99 (2001).

[97] K. Eguchi et al., Phys. Rev. Lett. 90, 021802 (2003) [hep-ex/0212021].

[98] T. Araki et al., Phys. Rev. Lett. 94, 081801 (2005) [hep-ex/0406035].

[99] K. Anderson et al., hep-ex/0402041.

[100] X. Guo et al. [Daya Bay Collaboration], hep-ex/0701029.

[101] F. Ardellier et al. [Double CHOOZ Collaboration], hep-ex/0606025.

[102] T. Lasserre e H. W. Sobel, Comptes Rendus Physique 6, 749 (2005) [nuclex/0601013].

[103] H. Kwon et al., Phys. Rev. D 24, 1097 (1981).

[104] G. Zacek et al., Phys. Rev. D 34, 2621 (1986).

[105] A. I. Afonin et al., Sov. J. Nucl. Phys. 46, 944 (1987).

[106] G. S. Vidyakin et al., JETP Lett. 59, 25 (1994).

[107] Z. D. Greenwood et al., Phys. Rev. D 53, 6054 (1996).

[108] M. Apollonio et al., Eur. Phys. J. C 27, 331 (2003). 
[109] F. Boehm et al., hep-ex/0107009.

[110] F. Reines et al., Phys. Rev. Lett. 15, 429 (1965); C. V. Achar et al., Phys. Lett. 18, 196 (1965).

[111] T. Kajita, New J. Phys. 6, 194 (2004).

[112] M. Honda, T. Kajita, K. Kasahara e S. Midorikawa, Phys. Rev. D 70, 043008 (2004).

[113] G. Barr et al., Phys. Rev. D 70, 023006 (2004).

[114] G. Battistoni et al., hep-ph/0305208.

[115] P. Adamson et al. [MINOS Collaboration], Phys. Rev. D 73, 072002 (2006).

[116] P. Adamson et al. [MINOS Collaboration], hep-ex/0701045.

[117] M. R. Krishnaswamy et al., Phys. Lett. B 106, 339 (1981).

[118] G. Battistoni et al., Nucl. Instrum. Meth. A 245, 277 (1986); M. Aglietta et al. [NUSEX Collaboration], Europhys. Lett. 8, 611 (1989).

[119] Ch. Berger et al., Nucl. Instrum. Meth. A 262, 463 (1987); K. Daum et al. [FREJUS Collaboration], Z. Phys. C 66, 417 (1995).

[120] W. W. M. Allison et al. [Soudan 2 Collaboration], Nucl. Instrum. Meth. A 376, 36 (1996); ibid. A 381, 385 (1996); ibid. Phys Lett. B 449, 137 (1999).

[121] E. N. Alekseev et al., Phys. Part. Nucl. 29, 254 (1998).

[122] M. Ambrosio et al. [MACRO Collaboration], Phys. Lett. B 517, 59 (2001) [hep-ex/0106049].

[123] D. Casper et al., Phys. Rev. Lett. 66, 2561 (1991); R. Becker-Szendy et al. [IMB Collaboration], Phys. Rev. D 46, 3720 (1992).

[124] K. S. Hirata et al. [Kamiokande Collaboration], Phys. Lett. B 280, 146 (1992).

[125] Y. Fukuda et al. [Kamiokande Collaboration], Phys. Lett. B 335, 237 (1994).

[126] Y. Fukuda et al. [Super-Kamiokande Collaboration], Phys. Lett. B 433, 9 (1998). 
[127] Y. Fukuda et al. [Super-Kamiokande Collaboration], Phys. Lett. B 436, 33 (1998).

[128] Palestra conferida por C. Walter no The XXXIII International Conference on High Energy Physics, Moscow, Rússia (2006).

[129] Veja relatórios e palestras em http://www.imsc.res.in/ ${ }^{\sim}$ ino .

[130] T. Tabarelli de Fatis, Eur. Phys. J. C 24, 43 (2002) [hep-ph/0202232].

[131] A. Geiser, Rept. Prog. Phys. 63, 1779 (2000).

[132] H. Abramowicz et al. [CDHS Collaboration], Nucl. Instrum. Meth. 180, 429 (1981). F. Dydak et al., Phys. Lett. B 134, 281 (1984).

[133] W. Sakamoto et al. [CCFR Collaboration], Nucl. Instrum. Meth. A 294, 179 (1990). T. Bolton et al. [NuTev Collaboration], Fermilab Proposal P815 (1990). A. Romosan et al., Phys. Rev. Lett. 78, 2912 (1997).

[134] E. Able et al. [MINOS Collaboration], Fermilab Proposal P875.

[135] D. Allasia et al., Nucl. Phys. B 307, 1 (1988).

[136] N. Ushida et al. [E531 Collaboration], Nucl. Instrum. Meth. 224, 50 (1984). N. Ushida et al., Phys. Rev. Lett. 57, 2897 (1986).

[137] E. Eskut et al. [CHORUS Collaboration], Nucl. Instrum. Meth. A 401, 7 (1997). E. Eskut et al., Phys. Lett. B 497, 8 (2001).

[138] K. Kodama et al., OPERA Progress Report CERN-SPSC/99-20, SPSC/M635, LNGS-LOI 19/99.

[139] D. Geiregat et al. [CHARM II Collaboration], Nucl. Instrum. Meth. A 325, 92 (1993).

[140] J. Altegoer et al. [NOMAD Collaboration], Nucl. Instrum. Meth. A 404, 96 (1998). P. Astier et al., Nucl. Phys. B 611, 3 (2001) [hep-ex/0106102].

[141] Neutrino Unbound site http://www.nu.to.infn.it/exp/sbl/ .

[142] L. Borodovsky et al. [E776 Collaboration], Phys. Rev. Lett. 68, 274 (1992).

[143] L. A. Ahrens et al. [E734 Collaboration], Phys. Rev. D 36, 702 (1987).

[144] B. Ambruster et al. [KARMEN Collaboration], Phys. Rev. D 65, 112001 (2002) [hep-ex/0203021]. 
[145] D. Naples et al. [CCFR/NuTev Collaboration], Phys. Rev. D 59, 031101 (1999) [hep-ex/9809023].

[146] K. S. McFarland et al., Phys. Rev. Lett. 75, 3993 (1995) [hep-ex/9506007].

[147] A. Aguilar et al. [LSND Collaboration], Phys. Rev. D 64, 112007 (2001) [hep-ex/0104049].

[148] A. Bazarko et al. [MiniBooNE Collaboration], Nucl. Phys. Proc. Suppl. 91, 210 (2001) [hep-ex/0009056].

[149] A. Aguilar-Arevalo et al., hep-ex/0704.1500.

[150] K. Nishikawa, Nucl. Phys. Proc. Suppl. 59, 289 (1997).

[151] S. H. Ahn et al. [K2K Collaboration], Phys. Lett. B 511, 178 (2001) [hepex/0103001].

[152] E. Aliu et al. [K2K Collaboration], Phys. Rev. Lett. 94, 081802 (2005) [hep-ex/0411038].

[153] D. G. Michael et al. [MINOS Collaboration], Phys. Rev. Lett. 97, 191801 (2006) [hep-ex/0607088].

[154] A. G. Cocco [OPERA Collaboration], Nucl. Phys. Proc. Suppl. 85, 125 (2000).

[155] R. Acquafredda et al. [OPERA Collaboration], New J. Phys 8, 303 (2006) [hep-ex/0611023].

[156] L. Bartoszek et al., hep-ex/0408121.

[157] P. Zucchelli, Phys. Lett. B 532, 166 (2002); B. Autin et al., J. Phys. G 29, 1785 (2003) [physics/0306106]; J. Burguet-Castell et al., Nucl. Phys. B 695, 217 (2004) [hep-ph/0312068]; J. Burguet-Castell et al., Nucl. Phys. B 725, 306 (2005) [hep-ph/0503021].

[158] S. Geer, hep-ph/9712290; A. De Rujula et al., hep-ph/9811390; C. H. Albright et al. [Neutrino Factory/muon Collider Collaboration], physics/0411123.

[159] T. K. Kuo e J. Pantaleone, Rev. Mod. Phys. 61, 937 (1989).

[160] T. K. Kuo e J. Pantaleone, Phys. Rev. D 39, 1930 (1989).

[161] H. A. Bethe, Phys. Rev. Lett. 56, 1305 (1986). 
[162] L. Landau, Phys. Z. Sowjetunion 2, 46 (1932).

[163] C. Zener, Proc. R. Soc. London A 137, 696 (1932).

[164] E. C. G. Stueckelberg, Helv. Phys. Acta 5, 369 (1932).

[165] S. J. Parke, Phys. Rev. Lett. 57, 1275 (1986).

[166] M. C. Gonzalez-Garcia e Y. Nir, Rev. Mod. Phys. 75, 345 (2003) [hep$\mathrm{ph} / 0202058]$.

[167] S. Fukuda et al. [Super-Kamiokande Collaboration], Phys. Rev. Lett. 85, 3999 (2000) [hep-ex/0009001].

[168] M. Maltoni e T. Schwetz, hep-ph/0705.0107.

[169] T. Yanagida, em Proc. of the Workshop on Unified Theories and Baryon Number in the Universe, Tsukuba, Japão, 1979, eds. O. Sawada e A. Sugamoto (KEK repor no. 79-18, Tsukuba, 1979); M. Gell-Mann, P. Ramond, e R. Slansky, em Supergravity, Proceedings of the Workshop, Stony Brook, New York, 1979, eds. F. van Nieuwenhuizen e D. Freedman (North Holland, Amsterdã, 1979); R. N. Mohapatra e G. Senjanovic, Phys. Rev. Lett. 44, 912 (1980).

[170] G. B. Gelmini \& M. Roncadelli, Phys. Lett. B 99, 411 (1981).

[171] A. Zee, Phys. Lett. B 93, 389 (1980).

[172] K. S. Babu, Phys. Lett. B 203, 132 (1988).

[173] T. P. Cheng \& L. F. Li, Phys. Rev. D 22, 2860 (1980).

[174] W. Grimus, hep-ph/0307149.

[175] J. Schechter e J. W. F. Valle, Phys. Rev. D 22, 2227 (1980); S. M. Bilenky, J. Hosek e S. T. Petcov, Phys. Lett. B 94, 495 (1980); I. Yu. Kobzarev, B. V. Martemyanov, L. B. Okun e M. G. Shchepkin, Yad. Phys. 32, 1590 (1980) [Sov. J. Nucl. Phys. 32, 823 (1981)].

[176] A. M. G. Medina, Soluções de grande comprimento de oscilação para o problema dos neutrinos solares, Tese de Doutorado, Universidade de São Paulo (2001).

[177] J. Erler \& P. Langacker, Phys. Lett. B 456, 68 (1999) [hep-ph/9903476].

[178] W. Grimus, R. Pfeiffer e T. Schwetz, Eur. Phys. J. C 13, 125 (2000) [hep$\mathrm{ph} / 9905320]$. 
[179] H. M. Georgi, S. L. Glashow e S. Nussinov, Nucl. Phys. B 193, 297 (1981).

[180] E. Ma \& U. Sarkar, Phys. Rev. Lett 80, 5716 (1998) [hep-ph/9802445].

[181] G. 't Hooft, Nucl. Phys B 33, 173 (1971); Nucl. Phys B 35, 167 (1971).

[182] L. Wolfenstein, Nucl. Phys. B 175, 93 (1980).

[183] P. H. Frampton \& S. L. Glashow, Phys. Lett. B 449, 240 (1999) [hep$\mathrm{ph} / 9906375]$.

[184] K. R. S. Balaji, W. Grimus e T. Schwetz, Phys. Lett. B 508, 301 (2001) [hep-ph/0104035].

[185] K. S. Babu \& C. Macesanu, Phys. Rev. D 67, 073010 (2003) [hep$\mathrm{ph} / 0112058]$.

[186] D. A. Sierra \& M. Hirsch, hep-ph/0609307.

[187] M. E. Peskin e D. V. Schroeder, em "An Introduction to Quantum Field Theory", Westview Press, 1995.

[188] A. Halprin, Phys. Rev. D 34, 3462 (1986).

[189] P. D. Mannheim, Phys. Rev. D 37, 1935 (1988).

[190] A. J. Baltz e J. Weneser, Phys. Rev. D 37, 3364 (1988).

[191] C. Chevalley, em "Theory of Lie Groups", Princeton University Press, 1946.

[192] S. Chaturvedi e N. Mukunda, hep-ph/0004219.

[193] H. Fritzsch e Z. Xing, hep-ph/9708366.

[194] L. L. Chau e W. Y. Keung, Phys. Rev. Lett. 53, 1802 (1984).

[195] F. Mandl e G. Shaw, em "Quantum Field Theory", John Wiley \& Sons, 1984.

[196] E. K. Akhmedov, R. Johansson, M. Lindner, T. Ohlsson e T. Schwetz, JHEP 0404, 078 (2004) [hep-ph/0402175].

[197] H. Lehmann, P. Osland e T. T. Wu, Commun. Math. Phys. 219, 77 (2001) [hep-ph/0006213].

[198] S. T. Petcov, Phys. Lett. B 191, 299 (1987). 
[199] T. Kaneko, Prog. Theor. Phys. 78, 532 (1987); M. Ito, T. Kaneko e M. Nakagawa, Prog. Theor. Phys. 79, 13 (1988).

[200] S. Toshev, Phys. Lett. B 196, 170 (1987).

[201] S. T. Petcov, Phys. Lett. B 200, 373 (1988).

[202] E. Torrente, hep-ph/9505209.

[203] D. Nötzold, Phys. Rev. D 36, 1625 (1987).

[204] P. Osland e T. T. Wu, Phys. Rev. D 62, 013008 (2000) [hep-ph/9912540].

[205] W. N. Bailey, em "Generalized Hypergeometric Series", Cambridge University Press, 1935.

[206] H. Bateman, em "Higher Transcendental Functions", Vol. I, McGraw-Hill, 1953.

[207] E. C. Oliveira, em "Funções Especiais com Aplicações", Livraria da Física, 2005.

[208] I. S. Gradshteyn, I. M. Ryzhik e A. Jeffrey, em "Table of Integrals, Series e Products", Academic Press, 2000.

[209] H. W. Zaglauer e K. H. Schwarzer, Z. Phys. C 40, 273 (1988).

[210] T. Ohlsson e H. Snellman, J. Math. Phys. 41, 2768 (2000) [hep$\mathrm{ph} / 9910546]$.

[211] K. Kimura, A. Takamura e H. Yokomakura, Phys. Rev. D 66, 073005 (2002) [hep-ph/0205295].

[212] O. Yasuda, hep-ph/0704.1531.

[213] M. Blennow e T. Ohlsson, J. Math. Phys. 45, 4053 (2004) [hep$\mathrm{ph} / 0405033]$.

[214] W. C. Haxton, Phys. Rev. Lett. 57, 1271 (1986).

[215] E. Kh. Akhmedov, M. Maltoni e A. Yu. Smirnov, Phys. Rev. Lett. 95, $211801(2005)$. 